\title{
Advances in hernia surgery
}

Citation for published version (APA):

Mommers, E. H. H. (2018). Advances in hernia surgery: preoperative, intraoperative, and postoperative developments in abdominal wall surgery. [Doctoral Thesis, Maastricht University]. Datawyse /

Universitaire Pers Maastricht. https://doi.org/10.26481/dis.20180329em

Document status and date:

Published: 01/01/2018

DOI:

10.26481/dis.20180329em

Document Version:

Publisher's PDF, also known as Version of record

\section{Please check the document version of this publication:}

- A submitted manuscript is the version of the article upon submission and before peer-review. There can be important differences between the submitted version and the official published version of record.

People interested in the research are advised to contact the author for the final version of the publication, or visit the DOI to the publisher's website.

- The final author version and the galley proof are versions of the publication after peer review.

- The final published version features the final layout of the paper including the volume, issue and page numbers.

Link to publication

\footnotetext{
General rights rights.

- You may freely distribute the URL identifying the publication in the public portal. please follow below link for the End User Agreement:

www.umlib.nl/taverne-license

Take down policy

If you believe that this document breaches copyright please contact us at:

repository@maastrichtuniversity.nl

providing details and we will investigate your claim.
}

Copyright and moral rights for the publications made accessible in the public portal are retained by the authors and/or other copyright owners and it is a condition of accessing publications that users recognise and abide by the legal requirements associated with these

- Users may download and print one copy of any publication from the public portal for the purpose of private study or research.

- You may not further distribute the material or use it for any profit-making activity or commercial gain

If the publication is distributed under the terms of Article $25 \mathrm{fa}$ of the Dutch Copyright Act, indicated by the "Taverne" license above, 


\section{Advances in hernia surgery}

Preoperative, intraoperative, and postoperative developments in abdominal wall surgery

Elwin H.H. Mommers 


\section{Colofon}

Advances in hernia surgery, preoperative, intraoperative, and postoperative developments in abdominal wall surgery by Elwin H.H. Mommers.

Printing of this thesis was supported by:

\section{Maastricht MUMC+}

Elkerliek Hospital Helmond

Catharine Hospital Eindhoven

BARD Davol

Dutch Hernia Society

Livit

\section{Maastricht UMC+}
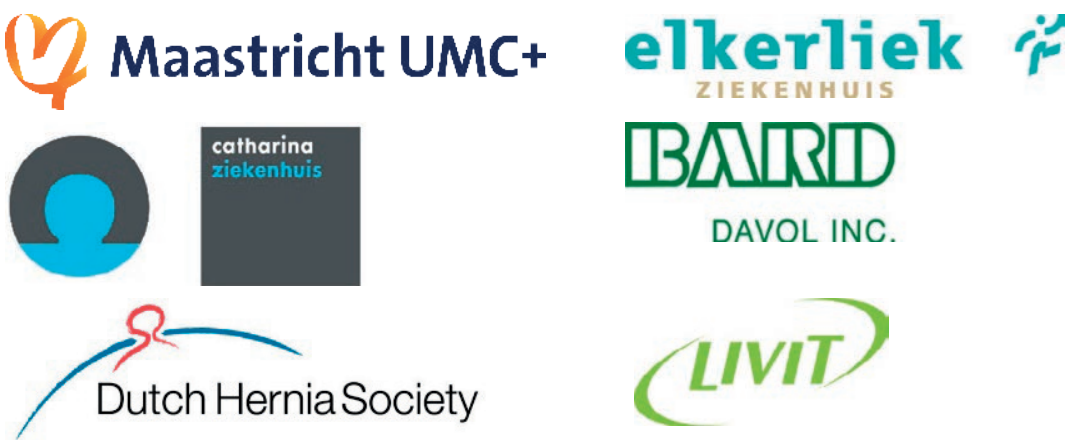

DAVOL INC.

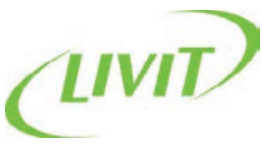

(C) Elwin H.H. Mommers 2017

ISBN: 9789461598080

Design \& Lay-out: Datawyse | Universitaire Pers Maastricht

Cover image: www.istockphoto.com

All rights reserved. No part of this thesis may be reproduced, stored, or transmitted in any form or by any means, electronic or mechanical, including photocopy, recording or any information storage or retrieval system, without prior permission of the copyright owner Elwin H.H. Mommers or the copyright-owning journals for previously published chapters and/or articles.

This thesis was prepared at the Department of School of Nutrition and Translational Research in Metabolism at the Maastricht University Medical Center \& Elkerliek Hospital Helmond. 


\title{
Advances in hernia surgery
}

\section{Preoperative, intraoperative, and postoperative developments in abdominal wall surgery}

\author{
PROEFSCHRIFT \\ Ter verkrijging van de graad van doctor aan de Universiteit Maastricht, \\ op gezag van de rector magnificus Prof. dr. Rianne M. Letschert, \\ volgens het besluit van het College van Decanen, \\ in het openbaar te verdedigen \\ op donderdag 29 Maart 2018 om 16:00 uur
}

door

Elwin Henri Hubert Mommers 


\section{Promotor}

Prof. Dr. Nicole D. Bouvy

\section{Copromotores}

Dr. Tammo S. de Vries Reilingh (Elkerliek ziekenhuis, Helmond)

Dr. Simon W. Nienhuijs (Catharina ziekenhuis, Eindhoven)

\section{Beoordelingscommissie}

Prof. Dr. Laurents P.S. Stassen (Voorzitter)

Dr. Theo J. Aufenacker (Rijnstate ziekenhuis, Arnhem)

Prof. Dr. Geerard L. Beets

Dr. Bibi M.E. Hansson (Canisius-Wilhelmina ziekenhuis, Nijmegen)

Prof. Dr. Harry C. Schouten 


\section{Table of Contents}

Chapter 1 Introduction and outline of this thesis

PART I PREOPERATIVE

Chapter 2 Can electric nose breath analysis identify abdominal wall hernia recurrence and aortic aneurysms? A proof of concept study

Chapter 3 Impact of hernia volume on pulmonary complications following complex hernia repair

PART II INTRAOPERATIVE

Chapter 4 How to perform the endoscopically assisted components separation technique (ECST) for large ventral hernia repair

Chapter 5 A modified Chevrel technique for ventral hernia repair: long term results of a single center cohort.

Chapter 6 Evaluating the 'standard' polypropylene and polyester mesh in terms of shrinkage, tissue ingrowth, and collagen deposition.

Chapter 7 Use of a bio absorbable mesh for complex ventral hernia repair in a contaminated field: a case report

Chapter 8 The general surgeon's perspective of rectus diastasis A systematic review of treatment options

PART III POSTOPERATIVE

Chapter 9 Patient-reported outcomes (PROs) after total extraperitoneal hernia repair (TEP)

Chapter 102016 EHS adhesion score: an international consensus based meshtissue adhesion score

Chapter 11 Complications and recurrence rates of patients with Ehlers-Danlos syndrome undergoing ventral hernioplasty

Chapter 12 Sex differences in baseline performance of the ischemic button model for induction of adhesions in laboratory rats 
Chapter 14 Summary

Summary (Dutch)

195

Acknowledgements / Dankwoord

199

List of publications

203

Curriculum Vitae

205

Valorisation

207 
Chapter

1

\section{Introduction and outline}


Chapter 1 


\section{INTRODUCTION AND OUTLINE}

The abdominal wall hernia is amongst the oldest diseases known to man. The earliest evidence of an abdominal wall hernia can be found in ancient terra cotta statues, dating back to the very first century. ${ }^{1}$ An abdominal wall hernia is defined as a protrusion of abdominal content through a defect in the abdominal musculature. ${ }^{2-4}$ The hernia is caused by an acquired or congenital weakness in the abdominal wall, causing the muscles to separate and the content to protrude outwards. ${ }^{4}$ A hernia consists of three key anatomical structures, namely the hernia sac, the hernia defect, and the hernia contents. Any type of abdominal wall hernia has a large impact on both the patient and the healthcare system in general., ${ }^{5,6}$ Hernias occur frequently with an overall prevalence of $1.7 \%$ in the general population. ${ }^{7}$ The incidence of specific abdominal wall hernias in descending order is inguinal, umbilical, epigastric, and incisional. This thesis mainly focusses on incisional hernias. ${ }^{8,9}$ Considering that $10-20 \%$ of all midline laparotomies eventually end in an incisional hernia, hernia repair is one of the most common surgical interventions. ${ }^{10,11}$ Despite common beliefs, hernia repair is not only an elective procedure, as approximately $10 \%$ of all hernia repairs are emergency procedures. ${ }^{12,13}$ There are two important groups of hernia related emergencies, the first is strangulation of the hernia contents, the second are complications caused by previous repairs such as mesh infections or fistulas. ${ }^{14}$

During the start of this thesis in 2013 the term 'complex hernia' was undefined and heterogenous, as was 'complex hernia repair'. The Elkerliek hospital in Helmond was the first hospital in The Netherlands that started creating a complex hernia pathway. The term 'complex' was based on a set of criteria that included both complicated hernia characteristics (width $\geq 10 \mathrm{~cm}$, lateral hernias, fistulas, etc.) as well as patient characteristics such as elaborate comorbidity, old age, impaired pulmonary function etc. During the following years, the criteria of Slater et al. replaced these criteria for complex hernias. ${ }^{15}$ All patients in the complex hernia pathway at the Elkerliek hospital were registered prospectively, received a CT-scan, and were discussed in a designated multidisciplinary 'complex hernia' meeting between multiple surgeons, a pulmonologist, radiologist, intensive care physician, and case manager. The unique approach of the Elkerliek hospital allowed for the creation of this thesis from their prospectively maintained database and uniform workup of patients. This thesis was written with the aim to address clinical problems encountered in the Elkerliek hospital, as well as carefully evaluate the current practice of modern hernioplasty with an attempt to make improvements where possible. The layout of the thesis addresses three stages of hernia management: preoperative, intraoperative, and postoperative care. 


\section{PREOPERATIVE}

Hernia care in the Netherlands is a developing field. ${ }^{16}$ The first stage of hernia management is the pre-operative stage. During this stage estimating the patients risk for complications is crucial in the shared decision-making process. The patient's risk factors are important to the evidence-based calculation of the complication and recurrence risk. ${ }^{17}$ In recent years, several studies have looked at the identification and quantification of these risk factors, which has led to the development of a mobile application which uses risk factors to quantify a patient's risk for complications called the Carolinas Equation for Determining Associated Risks (CeDAR) app from Carolina healthcare. Using this software, the complication risk for hernia patients can be exactly calculated based on known risk factors such as age, sex, BMI, smoking, obesity, and diabetes. ${ }^{18}$

Chapter two focusses on a novel type of risk assessment, the breatprint analysis. This type of analysis requires patients to breathe through an electronic nose (eNose), a small portable device that analyses volatile organic compounds (VOCs). Inside everyone's breath are hundreds of VOCs which are by-products or end-products of normal physiological processes. ${ }^{19,20}$ In case of disturbed metabolic processes, for instance in cancer patients, the eNose can recognise a specific VOC pattern in exhaled breath to identify the disease. This process can be used to detect certain diseases in a very early phase, or in this thesis, risk factors for collagen disorders of which the patient is not even aware. Currently, the electronic nose has been successfully trained to recognise six different types of cancer, tuberculosis, pulmonary hypertension, inflammatory bowel disease, chronic kidney disease, Parkinson's, and multiple sclerosis. ${ }^{21,22}$ This technology can help identify patients that are at risk for a (recurrent) hernia even before the primary hernia occurs. There is increasing evidence that both patients with aortic aneurysms as well as patients with recurrent incisional hernias have altered collagen turnover profiles. ${ }^{23,24}$ The technology used by the eNose is based on non-specific sensors, not on miniature gas chromatography, as one might expect. Instead an eNose is a small portable device that uses non-specific sensors, such as nanohairs, or metal redox based sensors to measure changes in (amongst others) resistance. These sensors measure minute differences in resistance at the surface of the sensor and create a three-dimensional graph based on the variables, resistance, time, and temperature. The graph on its own has no clinical meaning. Though several graphs combined will allow pattern recognition software within an automated neural network to recognise differences between the 'healthy' and the 'diseased' population. The use of non-specific sensors keeps production costs low and paves the way to mass production or even incorporation of the technology in existing portable devices such as mobile phones, smartwatches, laptops, and tablets.

Chapter three describes the first evidence for a new type of risk factor. Despite a multitude of prediction models for postoperative complications, the predictive value of these models is limited. ${ }^{25}$ This can be explained by the large number of variables influ- 
encing the operative outcome and complications risk of a patient. Hence, discovering new risk factors that can add to these prediction models lead to improved accuracy of the preoperative risk assessment. For many years surgeons have thought that bigger hernias lead to more complications. Though isolating the effect of hernia size proved difficult in the absence of exact measuring tools. ${ }^{26}$ Moreover, patients with large hernias tend to have other risk factors and comorbidities clouding the influence of the risk factor hernia size. To ascertain the influence of hernia size and subtract the influence of other risk factors, the first step was to accurately measure hernia volume using software that is normally applied for measuring tumour volume in response to radiation- or chemotherapy. This software allowed for accurate measurement of hernia volume and eventually the construction of a multivariate logistic regression model to isolate the influence of hernia volume without other confounding risk factors.

\section{INTRAOPERATIVE}

As hernias have a great impact on QoL, many patients are willing to undergo hernia repair if their physical state of being allows for operative correction. A watchful waiting strategy towards hernias appears to be relatively safe, though approximately $19 \%$ of ventral hernia patients will undergo elective hernia repair within 5 years. ${ }^{27,28}$ For inguinal hernias the watchful waiting strategy is less successful, as $23 \%$ of patients request elective repair within two years. ${ }^{29}$

Ventral hernia repair can be performed using many different methods. Experience has taught us that the use of surgical mesh is a necessity to create a durable repair in hernias larger than $3 \mathrm{~cm} .{ }^{30}$ The European Hernia Society developed a classification for ventral hernias that classifies a hernia based on size and location. Sizes vary between small $(<4 \mathrm{~cm})$, moderate $(4-10 \mathrm{~cm})$, and large $(\geq 10 \mathrm{~cm})$. The repair of 'large' hernias is particularly challenging due to the high complication rate, and the high risk for recurrences. This section of the thesis evaluates two surgical techniques for the management of larger ventral hernias. Chapter four describes how to perform an endoscopically assisted component separation technique (ECST) that can be used for ventral hernia repair of hernias up to $15 \mathrm{~cm}$. This technique is a modification of an open procedure described in 1990 by Ramirez et al. that releases the external oblique muscle from the aponeurosis at the anterior rectus fascia medialisation of the anterior and posterior rectus fascia to close the midline. ${ }^{31}$ The ECST was developed to decrease the number of surgical site occurrences (SSO), as the original Ramirez technique has a complication rate of approximately 35\%. This chapter contains a detailed explanation on how to perform ECST and reports an overall complication rate of 26\%. A meta-analysis of Switzer et al. confirmed that ECST has a lower complications rate compared to open component separation (CST). ${ }^{32}$ Chapter five describes a modified version of an onlay technique named after J.P. Chevrel that originated in the 1980 's. ${ }^{33,34}$ In short, this technique incis- 
es the anterior rectus fascia and turn it over to form a wider posterior rectus fascia. The gap in the anterior rectus fascia is then covered using an onlay mesh. The modification uses a smaller mesh that is trimmed to fit exactly inside the gap in the anterior rectus fascia with only minimal overlap. This modification needs less subcutaneous dissection and a smaller mesh, which could potentially reduce the amount of surgical site occurrences. Recently, the onlay repairs have become disfavoured due to reports of high complication and recurrence rates following onlay repair. ${ }^{13,35,36}$ These studies do not distinguish between different onlay techniques, hence the concerns proposed by the aforementioned studies regarding the onlay technique in general may not concern the modified Chevrel repair specifically. This chapter provides a detailed explanation of the surgical technique and analysed a single center, single surgeon cohort to identify the types and clinical relevance of postoperative complications after a modified Chevrel repair.

The success of ventral hernia repair is not only dependent on the technique used, though also on the type of mesh used. Chapter six evaluates 'standard' synthetic nonabsorbable meshes that are frequently used in ventral hernia repair. Despite seemingly comparable mesh characteristics these meshes have very different clinical performance. The chapter addresses the observed differences in mesh shrinkage, tissue ingrowth, inflammation, and collagen deposition in a subcutaneous rat model after 28 and 90 days follow-up. Alongside the previously mentioned non-absorbable synthetic meshes, there are many other types of surgical mesh available such as the synthetic absorbable mesh. These meshes have been developed to compete with the more expensive biological meshes and approach the 'ideal' characteristics of a mesh. Chapter seven describes a case in which a new type of synthetic absorbable mesh was used in complex hernia repair, complicated by an infected seroma on top of the mesh. A condition that frequently progresses to mesh infection, and subsequent removal of the mesh, one of the most feared postoperative complication after hernioplasty. The patient described in this chapter did not progress to the stage of mesh infection, which could be due to the use of a new type of synthetic absorbable mesh with specific characteristics that increase the mesh's resistance of antibiotic colonisation. ${ }^{37}$

Although the main topic of this thesis is abdominal wall hernia repair. Some patients may not even present with a hernia. Chapter eight describes the general surgeons approach to rectus diastasis. Despite the main topic of this thesis being ventral hernia repair, patients with rectus diastasis often seek medical intervention. Given the clinical similarity to ventral abdominal wall hernias, rectus diastasis patients are frequently referred to a surgeon for treatment. Given the lack of guidelines or even a systematic evaluation of the surgical treatment options for rectus diastasis chapter eight focusses on the surgical treatment options for rectus diastasis, as well as the potential efficacy of physiotherapy for rectus diastasis. ${ }^{38}$ Physiotherapy is a relatively new treatment modality for rectus diastasis and opinions about the efficacy are very diverse. ${ }^{39-48}$ Hence, the 
efficacy of physiotherapy for rectus diastasis was included as an outcome in the systematic literature review.

\section{POSTOPERATIVE}

After surgery, the most exciting part for the surgeon is over, though for the patient it has just begun. Scientific medical research, of which ventral abdominal wall hernia research is no exception, is most often performed by physicians. Hence it is to be expected that outcomes of medical research are usually relevant for the physician. For decades' hernia research has focused primarily on outcomes relevant for the physician, such as complications rates, recurrence rates, and the evaluation and development of new materials. Outcomes that are directly relevant for the patient, such as pain, functional impairment, and QoL have been ignored. ${ }^{49}$ Only recently the use of patient reported outcome measures (PROMs) is becoming increasingly important. Hernia related research is incorporating PROMs more frequently, though the amount of data on PROMs is insufficient to allow for incorporation in national and international guidelines. Even though, PROMs reflect outcomes that are directly measured and experienced by the patients. Therefore, PROMs are arguably the most important outcome for any scientific medical study. In chapter nine a new PROM questionnaire, the COMI-hernia questionnaire, is being used for the first time since its clinical validation by Staerkle et al. ${ }^{50}$ This chapter describes QoL in a population of inguinal hernia patients that were operated using a laparoscopic total extraperitoneal approach (TEP). QoL in the COMIhernia questionnaire is evaluated with a single question, as opposed to the more elaborate SF-36 questionnaire. ${ }^{50}$

Unfortunately, hernia repair is not free from risks, as with any surgical procedure postoperative complications occur. Chapter ten focusses on the unseen iceberg of postoperative complications, adhesions. Adhesions are fibrous connections between two or more structures in the abdomen that are not supposed to be connected. ${ }^{51-56}$ Particularly in repairs that result in direct contact between a mesh and the intra-abdominal content, adhesions are a feared complication. Adhesions occur in nearly all intra-abdominal procedures, though they are not always clinically relevant. ${ }^{57}$ Most patients will never develop complaints, though 5-18\% of the patients experience symptoms of bowel obstruction and $4 \%$ needs additional surgery to relieve them from their complaints or because the bowel becomes so obstructed that stool can't pass through normally. ${ }^{52,53,56}$ Another problem arises when patients are in need of new abdominal surgery later in life. Adhesions in the abdomen will make secondary surgery more difficult and increases the risk of complications. ${ }^{58}$ The cost associated with postoperative adhesions are estimated to be around 1.3 billion dollars per year in the United States of America. ${ }^{59,60}$ The systematic scoring of adhesions proves incredibly difficult and this has led to incomparable study outcomes. To address this problem a Delphi analysis was conducted using a 
panel of international expert in the field of intra-abdominal adhesions to create a consensus score for the measurement of intra-abdominal adhesion, the 2016 EHS consensus score (EHS name approval pending). This score is developed with the intention to increase interstudy comparability of adhesion research.

As mentioned previously, complications after hernia repair occur, though there are specific populations that are at risk for complications and recurrences. Chapter eleven focusses on the results of ventral hernia repair in an Ehlers Danlos syndrome (EDS) population. EDS impacts collagen quality and causes a wide variety of clinical symptoms. $^{61}$ It is expected that patients with EDS have a higher risk of surgical site occurrences and recurrences, though information regarding this vulnerable population is scarce. $^{62,63}$ To provide some insight in the results of ventral hernia repair in the EDS population a retrospective analysis of all patients with EDS operated in a large university hospital in Belgium were analysed.

Chapter twelve focusses on the experimental induction of adhesions. Postoperative adhesions occur after nearly all intra-abdominal procedures. Many different studies have attempted to reduce the incidence of postoperative adhesions, though promising results are scares. ${ }^{64-67}$ In most cases, new anti-adhesive devices are tested in animals before human application. To date, most animal studies are performed in male rats, reducing translatability to females. ${ }^{68}$ This chapter focusses on the ischemic button model, and attempts to refine the model by comparing the efficacy in male and female Whistar Han rats. 


\section{REFERENCE}

1. Zimmerman LM, Anson BJ. Anatomy and Surgery of Hernia: The williams @ Wilkins company Baltimore; 1953.

2. Korenkov M, Paul A, Sauerland S, et al. Classification and surgical treatment of incisional hernia. Results of an experts' meeting. Langenbeck's archives of surgery 2001;386:65-73.

3. Muysoms FE, Miserez M, Berrevoet F, et al. Classification of primary and incisional abdominal wall hernias. Hernia : the journal of hernias and abdominal wall surgery 2009;13:407-14.

4. Bendavid R. The unified theory of hernia formation. Hernia : the journal of hernias and abdominal wall surgery 2004;8:171-6.

5. Funk LM, Perry KA, Narula VK, Mikami DJ, Melvin WS. Current national practice patterns for inpatient management of ventral abdominal wall hernia in the United States. Surgical endoscopy 2013;27:4104-12.

6. Chung L, O'Dwyer PJ. Pain and its effects on physical activity and quality of life before operation in patients undergoing elective inguinal and ventral hernia repair. American journal of surgery 2014;208:406-11.

7. Beadles CA, Meagher AD, Charles AG. Trends in emergent hernia repair in the United States. JAMA surgery 2015;150:194-200.

8. Operaties in het ziekenhuis; soort opname, leeftijd en geslacht, 1995-2010. (Accessed 2-2-2017, 2017, at http://statline.cbs.nl/StatWeb/publication/?VW=T\&DM=SLNL\&PA=80386NED\&LA=NL.)

9. Dabbas N, Adams K, Pearson K, Royle GT. Frequency of abdominal wall hernias: is classical teaching out of date? JRSM Short Reports 2011;2:5.

10. Le Huu Nho R, Mege D, Ouaissi M, Sielezneff I, Sastre B. Incidence and prevention of ventral incisional hernia. Journal of visceral surgery 2012;149:e3-14.

11. Fink $C$, Baumann $P$, Wente $M N$, et al. Incisional hernia rate 3 years after midline laparotomy. The British journal of surgery 2014;101:51-4.

12. Helgstrand F, Rosenberg J, Bay-Nielsen M, et al. Establishment and initial experiences from the Danish Ventral Hernia Database. Hernia : the journal of hernias and abdominal wall surgery 2010;14:131-5.

13. Israelsson LA, Smedberg S, Montgomery A, Nordin P, Spangen L. Incisional hernia repair in Sweden 2002. Hernia : the journal of hernias and abdominal wall surgery 2006;10:258-61.

14. Fitzgibbons RJ, Jonasson O, Gibbs J, et al. The development of a clinical trial to determine if watchful waiting is an acceptable alternative to routine herniorrhaphy for patients with minimal or no hernia symptoms. Journal of the American College of Surgeons 2003;196:737-42.

15. Slater NJ, Montgomery A, Berrevoet F, et al. Criteria for definition of a complex abdominal wall hernia. Hernia : the journal of hernias and abdominal wall surgery 2014;18:7-17.

16. Wegdam JA; de Vries Reilingh TS; Nienhuijs SW. Complexe buikwandbreuk verdient aandacht. Medisch Contact 2015:3.

17. Dietz UA, Winkler MS, Hartel RW, et al. Importance of recurrence rating, morphology, hernial gap size, and risk factors in ventral and incisional hernia classification. Hernia : the journal of hernias and abdominal wall surgery 2014;18:19-30.

18. Dietz UA, Winkler MS, Härtel RW, et al. Importance of recurrence rating, morphology, hernial gap size, and risk factors in ventral and incisional hernia classification. Hernia : the journal of hernias and abdominal wall surgery 2014;18:19-30.

19. de Meij TG, Larbi IB, van der Schee MP, et al. Electronic nose can discriminate colorectal carcinoma and advanced adenomas by fecal volatile biomarker analysis: proof of principle study. International journal of cancer 2014;134:1132-8.

20. Amann A, Costello Bde L, Miekisch W, et al. The human volatilome: volatile organic compounds (VOCs) in exhaled breath, skin emanations, urine, feces and saliva. Journal of breath research 2014;8:034001.

21. Brooks SW, Moore DR, Marzouk EB, Glenn FR, Hallock RM. Canine olfaction and electronic nose detection of volatile organic compounds in the detection of cancer: a review. Cancer investigation 2015;33:411-9. 
22. Nakhleh MK, Amal H, Jeries R, et al. Diagnosis and Classification of 17 Diseases from 1404 Subjects via Pattern Analysis of Exhaled Molecules. ACS nano 2017;11:112-25.

23. Henriksen NA, Mortensen $\mathrm{JH}$, Sorensen LT, et al. The collagen turnover profile is altered in patients with inguinal and incisional hernia. Surgery 2015;157:312-21.

24. Rodella LF, Rezzani R, Bonomini F, et al. Abdominal aortic aneurysm and histological, clinical, radiological correlation. Acta histochemica 2016;118:256-62.

25. Mitchell TO, Holihan JL, Askenasy EP, et al. Do risk calculators accurately predict surgical site occurrences? J Surg Res 2016;203:56-63. doi: 10.1016/j.jss.2016.03.040. Epub Mar 26.

26. Tanaka EY, Yoo JH, Rodrigues AJ, Jr., Utiyama EM, Birolini D, Rasslan S. A computerized tomography scan method for calculating the hernia sac and abdominal cavity volume in complex large incisional hernia with loss of domain. Hernia : the journal of hernias and abdominal wall surgery 2010;14:63-9.

27. Ramanan B, Maloley BJ, Fitzgibbons RJ, Jr. Inguinal hernia: follow or repair? Advances in surgery 2014;48:1-11.

28. Kokotovic D, Sjolander H, Gogenur I, Helgstrand F. Watchful waiting as a treatment strategy for patients with a ventral hernia appears to be safe. Hernia : the journal of hernias and abdominal wall surgery 2016;20:281-7

29. Fitzgibbons RJ, Jr., Giobbie-Hurder A, Gibbs JO, et al. Watchful waiting vs repair of inguinal hernia in minimally symptomatic men: a randomized clinical trial. Jama 2006;295:285-92.

30. Silecchia G, Campanile FC, Sanchez L, et al. Laparoscopic ventral/incisional hernia repair: updated Consensus Development Conference based guidelines [corrected]. Surgical endoscopy 2015;29:2463-84.

31. Ramirez OM, Ruas E, Dellon AL. "Components separation" method for closure of abdominal-wall defects: an anatomic and clinical study. Plastic and reconstructive surgery 1990;86:519-26.

32. Switzer NJ, Dykstra MA, Gill RS, et al. Endoscopic versus open component separation: systematic review and meta-analysis. Surgical endoscopy 2015;29:787-95.

33. Chevrel JP. Traitment des grandes éventrations médianes par plastie en paletot et prothèse. La Nouvelle Presse Médicale 1979;8:695-6.

34. Chevrel JP, Morquette H. Traitment des éventrations abdominales médianes par autoplastie musculaire et prothèse pré-musculo-aponévrotique. Chirurgie (Mémoires de l'Académie) 1986;9:7.

35. Timmermans L, de Goede B, van Dijk SM, Kleinrensink GJ, Jeekel J, Lange JF. Meta-analysis of sublay versus onlay mesh repair in incisional hernia surgery. American journal of surgery 2014;207:980-8.

36. de Vries Reilingh TS, van Geldere D, Langenhorst B, et al. Repair of large midline incisional hernias with polypropylene mesh: comparison of three operative techniques. Hernia : the journal of hernias and abdominal wall surgery 2004;8:56-9.

37. Deeken CR, Matthews BD. Characterization of the Mechanical Strength, Resorption Properties, and Histologic Characteristics of a Fully Absorbable Material (Poly-4-hydroxybutyrate-PHASIX Mesh) in a Porcine Model of Hernia Repair. ISRN surgery 2013;2013:238067.

38. Akram J, Matzen SH. Rectus abdominis diastasis. Journal of plastic surgery and hand surgery 2014;48:163-9.

39. Acharry N, Kutty RK. Abdominal Exercise with Bracing, a Therapeutic Efficacy in Reducing Diastasis-Recti among Postpartal Females. International Journal of Physiotherapy and Research 2015;3:999-1005.

40. Gallus KM, Golberg KF, Field R. Functional Improvement Following Diastasis Rectus Abdominus Repair in an Active Duty Navy Female. Military medicine 2016;181:e952-4.

41. Kh SR, ale, H D. Effects of Abdominal Exercises on Reduction of Diastasis Recti in Postnatal Women. IJHSR 2016;6:182-91.

42. Chiarello CM, Falzone LA, McCaslin KE, Patel MN, Ulery KR. The Effects of an Exercise Program on Diastasis Recti Abdominis in Pregnant Women. Journal of Women's Health Physical Therapy 2005;29:11-6.

43. Keeler J, Albrecht M, Eberhardt L, Horn L, Donnelly C, Lowe D. Diastasis Recti Abdominis: A Survey of Women's Health Specialists for Current Physical Therapy Clinical Practice for Postpartum Women. Journal of Women's Health Physical Therapy 2012;36:131-42.

44. Litos K. Progressive Therapeutic Exercise Program for Successful Treatment of a Postpartum Woman With a Severe Diastasis Recti Abdominis. Journal of Women's Health Physical Therapy 2014;38:58-73. 
45. Mesquita LA, Machado AV, Andrade AV. Fisioterapia para redução da diástase dos músculos retos abdominais no pós-parto. Revista Brasileira de Ginecologia e Obstetrícia 1999;21:267-72.

46. Sharma G, Lobo T, Keller L. Postnatal Exercise Can Reverse Diastasis Recti. Obstetrics \& Gynecology 2014;123:171S.

47. Sheppard S. The Role of Transversus Abdominus in Post Partum Correction of Gross Divarication Recti. Manual Therapy 1996;1:214-6.

48. Walton LM, Costa A, LaVanture D, Mcllrath S, Stebbins B. The effects of a 6 week dynamic core stability plank exercise program compared to a traditional supine core stability strengthening program on diastasis recti abdominis closure, pain, oswestry disability index (ODI) and pelvic floor disability index scores (PFDI). Physical Therapy and Rehabilitation 2016;3:3.

49. Antonescu I, Mueller CL, Fried GM, Vassiliou MC, Mayo NE, Feldman LS. Outcomes reported in highimpact surgical journals. The British journal of surgery 2014;101:582-9.

50. Staerkle RF, Villiger P. Simple questionnaire for assessing core outcomes in inguinal hernia repair. The British journal of surgery 2011;98:148-55

51. Arung W, Meurisse M, Detry O. Pathophysiology and prevention of postoperative peritoneal adhesions. World J Gastroenterol 2011;17:4545-53.

52. Ellis H, Moran BJ, Thompson JN, et al. Adhesion-related hospital readmissions after abdominal and pelvic surgery: a retrospective cohort study. Lancet 1999;353:1476-80.

53. Hellebrekers BW, Kooistra T. Pathogenesis of postoperative adhesion formation. Br J Surg 2011;98:150316. doi: 10.002/bjs.7657. Epub 2011 Aug 23.

54. Mais V. Peritoneal adhesions after laparoscopic gastrointestinal surgery. World J Gastroenterol 2014;20:4917-25. doi: 10.3748/wjg.v20.i17.4917.

55. Schreinemacher MHF. Abdominal Wall Hernia Repair - Intraperitoneal Mesh and Adhesions. http://www.nvec.nl/wp-content/uploads/2015/05/Commissie-Abdominal-Wall-Hernia-Repair1-M.-

Schreinemacher.pdf: Maastricht University; 2015.

56. Wilson MS. Practicalities and costs of adhesions. Colorectal Dis 2007;9:60-5.

57. Ouaissi M, Gaujoux S, Veyrie N, et al. Post-operative adhesions after digestive surgery: their incidence and prevention: review of the literature. Journal of visceral surgery 2012;149:e104-14.

58. Bruggmann D, Tchartchian G, Wallwiener M, Munstedt K, Tinneberg HR, Hackethal A. Intra-abdominal adhesions: definition, origin, significance in surgical practice, and treatment options. Deutsches Arzteblatt international 2010;107:769-75.

59. Ray Ms NF, Denton Rn MBAWG, Thamer PhD M, Henderson Ms SC, Perry Md MS. Abdominal Adhesiolysis: Inpatient Care and Expenditures in the United States in 1994. Journal of the American College of Surgeons 1998;186:1-9.

60. Kössi J, Salminen P, Rantala A, Laato M. Population-based study of the surgical workload and economic impact of bowel obstruction caused by postoperative adhesions. British Journal of Surgery 2003;90:1441-4.

61. Henriksen NA. Systemic and local collagen turnover in hernia patients. Danish medical journal 2016;63.

62. Antoniou GA, Georgiadis GS, Antoniou SA, Granderath FA, Giannoukas AD, Lazarides MK. Abdominal aortic aneurysm and abdominal wall hernia as manifestations of a connective tissue disorder. Journal of vascular surgery 2011;54:1175-81.

63. Rosch R, Junge K, Knops M, Lynen P, Klinge U, Schumpelick V. Analysis of collagen-interacting proteins in patients with incisional hernias. Langenbeck's archives of surgery 2003;387:427-32.

64. Kuhry E, Schwenk WF, Gaupset R, Romild U, Bonjer HJ. Long-term results of laparoscopic colorectal cancer resection. The Cochrane database of systematic reviews 2008:Cd003432.

65. Hindocha A, Beere L, Dias S, Watson A, Ahmad G. Adhesion prevention agents for gynaecological surgery: an overview of Cochrane reviews. The Cochrane database of systematic reviews 2015;1:Cd011254.

66. Ahmad G, O'Flynn H, Hindocha A, Watson A. Barrier agents for adhesion prevention after gynaecological surgery. Cochrane Database Syst Rev 2015:CD000475. doi: 10.1002/14651858.CD000475.pub3.

67. Ahmad G, O'Flynn H, Hindocha A, Watson A. Barrier agents for adhesion prevention after gynaecological surgery. The Cochrane database of systematic reviews 2015:Cd000475.

68. Zucker I, Beery AK. Males still dominate animal studies. Nature 2010;465:690. 

PART

PREOPERATIVE 



\section{Chapter}

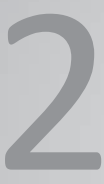

\section{Can electric nose breath analysis identify abdominal wall hernia recurrence and aortic aneurysms? A proof of concept study}

Elwin H.H. Mommers ${ }^{1}$, Lottie van Kooten ${ }^{1}$, Simon W. Nienhuijs ${ }^{2}$, Tammo S. de Vries Reilingh ${ }^{3}$, Stéphanie O. Breukink ${ }^{1}$, Barend M.E. Mees ${ }^{4}$, Geert -Willem H. Schurink ${ }^{4}$, Nicole D. Bouvy ${ }^{1}$

\footnotetext{
${ }^{1}$ Department of Surgery, Maastricht University Medical Center, Maastricht, The Netherlands ${ }^{2}$ Department of Surgery, Catharina Hospital, Eindhoven, The Netherlands ${ }^{3}$ Department of Surgery, Elkerliek Hospital, Helmond, The Netherlands ${ }^{4}$ Department of Vascular Surgery, Maastricht University Medical Center, Maastricht, The Netherlands
} 


\section{ABSTRACT}

Background: Hernia recurrence is observed in up to $32 \%$ of all ventral hernia patients. Previous studies have shown that patients who develop hernia recurrences are likely to have a decreased collagen I:III ratio. The same observation is made in patients with an aortic aneurysm, predisposing both for incisional and recurrent ventral hernia formation. If patients with weak collagen could be identified in a reliable, low cost, noninvasive manner, it would greatly enhance preventive options such as prophylactic mesh placement after abdominal surgery. The purpose of this study is to evaluate if an electric nose (Aeonose ${ }^{\circledR}$, the eNose company) can distinguish recurrent hernia patients and aortic aneurysm patients from healthy controls based on volatile organic compound analysis.

Methods: From February to July 2017 a three-armed proof of concept study was conducted at three hospitals, in three groups of patients; recurrent ventral hernia, aortic aneurysm, and healthy controls. Patients were measured once at the outpatient clinic using an electronic nose based on three metal oxide sensors. Measurement data were compressed to low-dimensional vectors using a TUCKER 3 like algorithm and used to train an artificial neural network (ANN) to provide a classification between patients $(+1)$ and healthy controls (-1).

Results: A total of 64 patients (Hernia $n=29$, Aneurysm $n=35$ ) and 37 controls were included in the study. Based on receiver operating curve (ROC) analysis, the ANN could differentiate between recurrent hernia patients and controls with the following details: area under the curve $(A \cup C) 0.74$, sensitivity 0.79 , specificity 0.65 . Aortic aneurysm patients and healthy controls could be differentiated with an AUC of 0.84 , sensitivity of 0.83 , and specificity of 0.81 .

Conclusions: The Aeonose ${ }^{\circledR}$ eNose can reliably distinguish patients with weak collagen (recurrent hernia and aortic aneurysm patients) from healthy controls. Validation of these results in a prospective cohort study is required before clinical application of the device. 


\section{INTRODUCTION}

Hernia recurrence is observed in up to $32 \%$ of the ventral hernia patients, depending on the complexity and size of the hernia, method of repair, and experience of the surgeon. ${ }^{1-3}$ Previous studies have shown that patients who develop hernia recurrences are likely to have an altered collagen I:III ratio and abnormal collagen IV and V turnover rates, predisposing them for hernia formation and hernia recurrence. ${ }^{4,5}$ Aortic aneurysm patients have similar collagen remodeling alteration as recurrent hernia patients. ${ }^{6,7}$ Identifying patients with weak collagen before abdominal surgery can be an indication for preventive mesh placement. As is becoming standard practice after open aortic aneurysm repairs. ${ }^{8-10}$

Analysis of volatile organic compounds (VOCs) in exhaled breath using an electronic nose (eNose) could potentially identify patients with weak collagen who are at risk for hernia formation preoperative. VOCs are end products of metabolic processes that are exhaled and can be measured to identify abnormalities in metabolic processes and subsequently, diagnose diseases. Exhaled breath contains approximately 850 different VOCs that form a volatile chemical breathprint. ${ }^{11}$ Previous studies have used eNose VOC analysis for the prediction of several types of cancer, COPD exacerbation with and without underlying pulmonary infection, asthma, sarcoidosis, cystic fibrosis, multiple sclerosis, Parkinson's disease, Alzheimer's disease, and many more. ${ }^{12}$ Previous studies have shown that the eNose is most sensitive for tissues with good blood perfusion such as the brain, liver, kidneys. ${ }^{5,7,13-15}$ A measurable alteration in this study is expected due to the good perfusion of the aorta and the abundance of collagen throughout the human body.

The eNose was first introduced in the 1970's as an alternative to gas chromatography and mass spectrometry. Chromatography and spectrometry have the advantage of qualitative analysis, precisely measuring the concentration of individual VOCs. The eNose uses non-specific measurements, analysing VOCs through pattern recognition algorithms and self-learning artificial neural networks (ANN). Essentially the eNose uses the entire collective of exhaled VOCs by analysing minute differences in surface conductivity of metal-oxide sensors caused by redox-reactions. The device collects thousands of data points from each patient that (after data compression) are used to train an ANN to distinguish patients from healthy controls. In this study, the ANN is trained using patients with impaired collagen quality (recurrent hernia and aortic aneurysm patients) and patients with good collagen quality (healthy controls). If the eNose can be trained to reliably distinguish these groups, it could be the first step towards preoperative identification of patients for preventive mesh placement. The aim of this study is to train the Aeonose $^{\circledR}$ eNose to distinguish recurrent ventral hernia patients and aortic aneurysm patients (separately) from healthy controls. 


\section{METHODS}

This trial was registered in the Dutch Trial Registry under number [nr.: NTR5954]. Approval from the Medical Ethics Committee was obtained before the start of this study [nr.: 164142]. From February to July 2017 a three-armed observational study including patients with a (history of) aortic aneurism, patients with a (history of) ventral hernia recurrence, and healthy controls was conducted. Inclusion criteria were any patient between 18 and 80 years old with a ventral hernia recurrence (current or in the past), or an aortic abdominal (diameter $>30 \mathrm{~mm}$ ) or thoracic aneurysm (diameter $>40 \mathrm{~mm}$ ) currently or after open or endovascular repair in the past.

Exclusion criteria were: patients with a parastomal hernia (recurrent hernia arm only), rectus diastasis without true hernia (recurrence hernia arm only), recurrent hernias with obvious cause (for instance mesh infection or traumatic hernias), Ehlers Danlos (all types), other collagen disorders (i.e. Marfan, Alport, Hypochondrogenesis, Bethlem myopathy, Fuch's dystrophy, etc.), clinical hyper- or hypothyroidism, severe COPD, or any type of cancer.

Informed consent was obtained from all individual participants included in this study. Patients were recruited non-consecutively at allocated days from the outpatient clinic of three participating hospitals in the Netherlands, one local hospital (Elkerliek Hospital, Helmond), one regional teaching hospital (Catharina Hospital, Eindhoven) and one university hospital (Maastricht University Medical Center, Maastricht). All patients were included by a group of three researches who performed inclusions at all sites.

Measurements were taken using the Aeonose ${ }^{\circledR}$ electronic nose (The eNose company, Zutphen, The Netherlands). A total of two different devices were used for this study. Each device had to include at least five positive and five negative cases to minimize measurement bias. For 5 minutes, patients breathe gently through a disposable mouthpiece with HEPA filter to protect the eNose from viruses and bacteria. A carbon filter filters the inhaled air, and a nose clip is used to ensure participant breath through the device. The details of the Aeonose ${ }^{\circledR}$ device, measurements, and data analysis have been described previously by Van Geffen et al. and Kort et al. ${ }^{16,17}$ In short, the device uses an array of non-specific hotplate-mounted metal-oxide sensors to measure real-time conductivity changes of the sensor surface, caused by redox reactions of VOCs. These conductivity changes are dependent on VOC composition, temperature, dynamics, and sensor surface properties. The sensors are guided through a sinusoidal temperature profile.

The first 2 minutes of breathing are used for rinsing the participant lungs with clean air. During the next 3 minutes, conductivity data are recorded. After that, sensors are regenerated using clean air, followed by heating a Tenax-tube. In that way, trapped VOC's are guided over the sensors. Finally, the sensors are regenerated again. Using a TUCKER3-like solution, data are compressed to a low dimensional vector per participant. $^{18}$ These vectors together with accompanying classification are used for training the ANN. ${ }^{19}$ Previous studies with the Aeonose ${ }^{\circledR}$ have shown that a minimal group size of 
approximately 25 patients per group is needed to train the artificial neural network reliably. ${ }^{16}$ To make sure training was on the disease and not on artefacts, cross validation was applied using the 'Leave-10\%-Out' method. In that way, ten models were trained using $90 \%$ of the data, and $10 \%$ as control to validate the outcome of the model. The $10 \%$ used for validating the ANN varied for each model. Target inclusion was set at 25 patients per group and limited at a maximum of 50 patients per group. Statistical analysis was performed with SPSS version 22, SPSS Statistics for Windows, IBM corp. Armonk, NY, released 2013. Baseline characteristics were analysed using descriptive statistics and compared between groups using independent sample student's T-test or Man-Whitney-U depending on the normal distribution of data.

\section{RESULTS}

A total of 64 patients (Hernia $n=29$, Aneurysm $n=35$ ) and 37 controls were included in this study (see table 1 for demographic characteristics). Three patients were excluded because they were unable to finish the measurement due to difficulty breathing through the device.

\section{Recurrent hernia versus control}

For this analysis 29 recurrent hernia patients, and 37 healthy controls were included. The results of ANN's ability to differentiate between recurrent hernia patients and healthy controls are presented in a receiver operating curve (ROC) (figure 1). The ROC has a Matthews correlation coefficient of 0.44 and reports a sensitivity of 0.79 , specificity of 0.65 , positive predictive value (PPV) of 0.64 , and negative predictive value (NPV) of 0.80 .

\section{Aortic aneurysm versus control}

For this analysis 35 aortic aneurysm patients, and 26 controls were included. Compared to the hernia analysis, eleven control patients were excluded from the aortic aneurysm analysis to prevent measurement bias as they were measured on an eNose device that was not used for measuring aortic aneurysm patients. Based on the ROC (figure 2) a Matthews correlation coefficient of 0.63 , sensitivity of 0.83 , specificity of 0.81 , PPV of 0.85 , and NPV of 0.78 were achieved. 
Chapter 2

Table 1 Demographic and perioperative details

\begin{tabular}{llll}
\hline Demographic characteristics & Aneurysm $(\mathrm{n}=35)$ & Hernia $(\mathrm{n}=29)$ & Control $(\mathrm{n}=37)$ \\
\hline Gender (male/female) & $28 / 7^{*}$ & $20 / 9^{*}$ & $14 / 23$ \\
Age $(\mathrm{yr})$, median (range) & $73(57-87)$ & $66(45-85)$ & $62(24-87)$ \\
$\mathrm{BMI}\left(\mathrm{kg} / \mathrm{m}^{2}\right)$ mean $(\mathrm{SD})$ & $26(19-38)$ & $28(21-43)$ & $24(15-33)$ \\
Smoking & $11(31 \%)^{*}$ & $7(24 \%)^{*}$ & $2(5 \%)$ \\
Alcohol within 48 hours & $10(29 \%)$ & $9(31 \%)$ & $14(37 \%)$ \\
Aneurism repair & $16(46 \%)$ & & \\
\hline
\end{tabular}

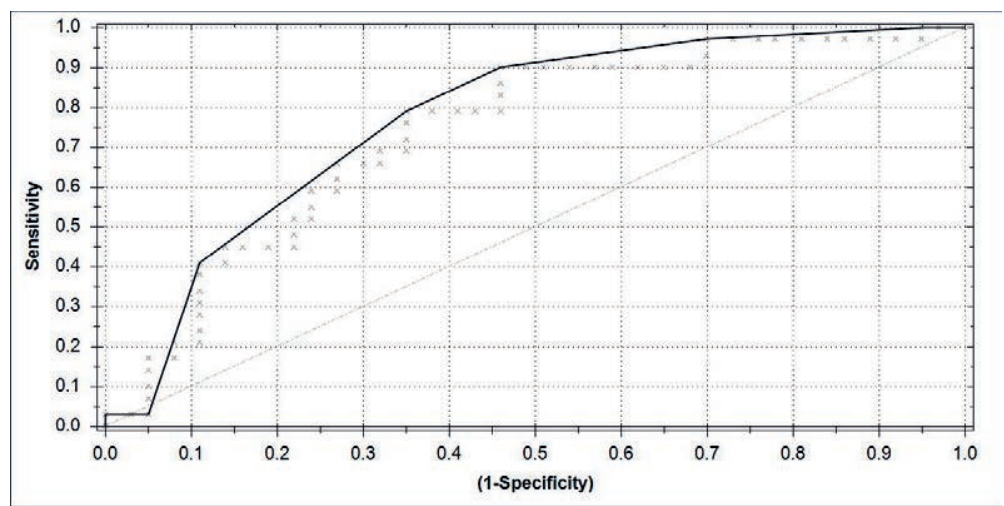

Figure 1 ROC recurrent hernia vs control

Area under the curve: 0.74. Matthews correlation coefficient: 0.44.

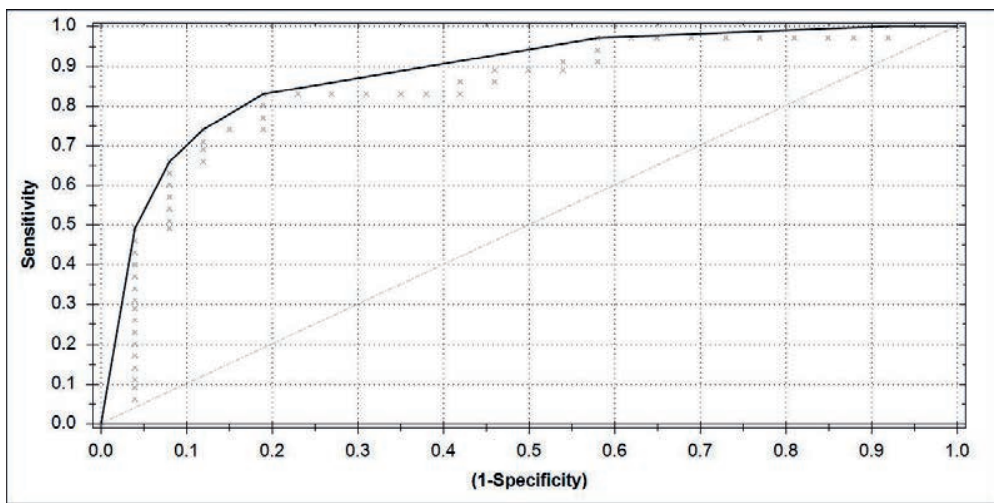

Figure 2 ROC aortic aneurysm vs control

Area under the curve: 0.84. Matthews correlation coefficient: 0.63. 


\section{DISCUSSION}

The Aeonose ${ }^{\circledR}$ eNose can reliably distinguish recurrent hernia patients and aortic aneurysm patients from healthy controls. The results of this study are the first step in identifying patients that could benefit from preventive mesh placement after abdominal surgery.

This study describes the first application of an eNose device to distinguish patients with low collagen quality (aortic aneurysms and recurrent hernia patients) from healthy controls. Both recurrent hernia and aortic aneurysm patients are thought to have impaired collagen quality, predisposing the to (recurrent) hernia formation. ${ }^{4,5}$ To reduce the incidence of incisional hernia formation, high risk patients must be identified preoperatively so preventive steps such as prophylactic mesh placement or stronger suture techniques can be implemented.

Application of an eNose in the field of ventral hernia repair and aortic aneurysms is new, the number of eNose diagnostics for both pulmonary and extra-pulmonary diseases has increased dramatically over the past years. Detection of colorectal cancer using electronic noses has been well established through alterations measured in both eNose, as well as gas chromatography and mass-spectrometry studies. ${ }^{20}$ Though similar qualitative studies are lacking in the field of ventral hernia repair and aortic aneurysm repair, the strength of the eNose analysis lies in the non-specific measurement. Identifying one or several VOCs linked to a particular disease is a complex process as most VOCs are the end product of multiple metabolic processes, hence they are seldom linked to one specific pathway. ${ }^{11}$ Instead of measuring one specific VOC, for instance isoprene as a marker for cholesterol biosynthesis, or ammonia for microbiota detection, the eNose measures the collective of the entire VOC breathprint. ${ }^{21,22}$ The ANN is able to 'learn' to distinguish patients from controls based on pattern recognition, hence the eNose can be applied in any type of disease that is expected to alter VOC composition, without knowing the exact metabolic changes caused by the disease. Herein lies both the strength and weakness of the eNose, as it is not possible to identify the discriminating factors the ANN uses to categorise patients and controls. The obtained AUC's and Matthews correlation coefficients observed in this study indicate that the eNose can distinguish both recurrent hernia and aortic aneurysm patients well from healthy controls. Before the eNose can be used in clinical practice a prospective cohort study must evaluate if the training results obtained in this study can be used for preoperative classification.

Three patients were unable to complete the eNose measurement due to shortness of breath. The eNose requires only gently breathing through the device, though the HEPA and carbon filters create an inhalation resistance which can be problematic for patient with asthma or pulmonary comorbidity. 


\section{Limitations}

This proof-of-concept study has included a limited number of patients and controls, adding to the number of patients will increase the accuracy of the results obtained from the ANN, as outliers and variations in a single value can have a great effect on the ANN's ability to distinguish patients. To train the ANN correctly, controls must be proven negative for the outcome disease. In this proof-of-concept study control patients did not receive imaging to verify the absence of an aortic aneurysm. There are numerous factors that could influence VOC composition, such as age, lung function, food, exercise, sleep, smoking, alcohol consumption etc. which can't be controlled in a small proof of concept study. These factors will have less influence in larger follow-up studies as the increased number of patients will ensure an equal distribution of confounders. ${ }^{12}$ Though variations in VOC composition in a small portion of the population, such as the statistically significant difference in smoking between the positive and negative group in this study are expected to have only limited influence as the ANN searched for one specific pattern in all positive cases. If $31 \%$ of the population has VOC alterations specific for smoking, the observed AUC of 0.84 and Matthews Correlation Coefficient of 0.63 can't be based on the 'smoking pattern'. The results obtained during this study were obtained using hotplate metal oxide sensor arrays, if the results can be replicated on different eNose sensory devices (Quartz microbalance, colorimetric sensors, etc.) remains to be seen. Despite the previously mentioned limitations, the authors believe that the following conclusion can be drawn from this study.

\section{CONCLUSION}

Using the Aeonose ${ }^{\circledR}$ eNose and artificial neural network analysis it was possible to reliably distinguish recurrent hernia and aortic aneurysm patients from healthy controls. Before clinical application of the eNose to identify patients suitable for prophylactic mesh placement after abdominal surgery, the results must be tested in a cohort study to evaluate if the training results can be used for preoperative classification. The current concept can be an important step forward in the identification of patients for prophylactic mesh placement.

\section{ACKNOWLEDGMENTS}

The authors would like to thank the supporting staff of the eNose company for their logistical support. 


\section{ETHICAL APPROVAL}

All procedures performed in studies involving human participants were in accordance with the ethical standards of the institutional and/or national research committee and with the 1964 Helsinki declaration and its later amendments or comparable ethical standards. 


\section{REFERENCES}

1. de Vries Reilingh TS, van Goor H, Rosman C, et al. "Components separation technique" for the repair of large abdominal wall hernias. J Am Coll Surg 2003;196:32-7.

2. Deerenberg EB, Timmermans L, Hogerzeil DP, et al. A systematic review of the surgical treatment of large incisional hernia. Hernia 2015;19:89-101. doi: 10.1007/s10029-014-1321-x. Epub 2014 Nov 8.

3. Israelsson LA, Smedberg S, Montgomery A, Nordin P, Spangen L. Incisional hernia repair in Sweden 2002. Hernia 2006;10:258-61.

4. Bendavid R. The unified theory of hernia formation. Hernia 2004;8:171-6.

5. Henriksen NA, Mortensen JH, Sorensen LT, et al. The collagen turnover profile is altered in patients with inguinal and incisional hernia. Surgery 2015;157:312-21.

6. Miner GH, Costa KD, Hanss BG, Marin ML. An Evolving Understanding of the Genetic Causes of Abdominal Aortic Aneurysm Disease. Surgical technology international 2015;26:197-205.

7. Rodella LF, Rezzani R, Bonomini F, et al. Abdominal aortic aneurysm and histological, clinical, radiological correlation. Acta histochemica 2016;118:256-62.

8. Bevis PM, Windhaber RA, Lear PA, Poskitt KR, Earnshaw JJ, Mitchell DC. Randomized clinical trial of mesh versus sutured wound closure after open abdominal aortic aneurysm surgery. The British journal of surgery 2010;97:1497-502.

9. Muysoms FE, Detry O, Vierendeels T, et al. Prevention of Incisional Hernias by Prophylactic Meshaugmented Reinforcement of Midline Laparotomies for Abdominal Aortic Aneurysm Treatment: A Randomized Controlled Trial. Annals of surgery 2016;263:638-45.

10. Nieuwenhuizen J, Eker HH, Timmermans L, et al. A double blind randomized controlled trial comparing primary suture closure with mesh augmented closure to reduce incisional hernia incidence. BMC surgery 2013;13:48.

11. Amann A, Costello Bde L, Miekisch W, et al. The human volatilome: volatile organic compounds (VOCs) in exhaled breath, skin emanations, urine, feces and saliva. Journal of breath research 2014;8:034001.

12. Bikov A, Lazar Z, Horvath I. Established methodological issues in electronic nose research: how far are we from using these instruments in clinical settings of breath analysis? Journal of breath research 2015;9:034001.

13. Harrison B, Sanniec K, Janis JE. Collagenopathies-Implications for Abdominal Wall Reconstruction: A Systematic Review. Plast Reconstr Surg Glob Open 2016;4:e1036.

14. Carmo M, Colombo L, Bruno A, et al. Alteration of elastin, collagen and their cross-links in abdominal aortic aneurysms. European journal of vascular and endovascular surgery : the official journal of the European Society for Vascular Surgery 2002;23:543-9.

15. Baxter BT, Davis VA, Minion DJ, Wang YP, Lynch TG, McManus BM. Abdominal aortic aneurysms are associated with altered matrix proteins of the nonaneurysmal aortic segments. Journal of vascular surgery 1994;19:797-802; discussion 3.

16. van Geffen WH, Bruins M, Kerstjens HA. Diagnosing viral and bacterial respiratory infections in acute COPD exacerbations by an electronic nose: a pilot study. J Breath Res 2016;10:036001. doi: 10.1088/1752-7155/10/3/.

17. Kort S, Brusse-Keizer M, Gerritsen JW, van der Palen J. Data analysis of electronic nose technology in lung cancer: generating prediction models by means of Aethena. Journal of breath research 2017;11:026006.

18. Kroonenberg PM. Applied Multiway Data Analysis: John Wiley \& Sons, Inc.; 2007.

19. Cartwright HM. Artificial neural networks in biology and chemistry: the evolution of a new analytical tool. Methods in molecular biology (Clifton, NJ) 2008;458:1-13.

20. de Boer NK, de Meij TG, Oort FA, et al. The scent of colorectal cancer: detection by volatile organic compound analysis. Clinical gastroenterology and hepatology : the official clinical practice journal of the American Gastroenterological Association 2014;12:1085-9. 
21. Salerno-Kennedy R, Cashman KD. Potential applications of breath isoprene as a biomarker in modern medicine: a concise overview. Wiener klinische Wochenschrift 2005;117:180-6.

22. Kearney DJ, Hubbard T, Putnam D. Breath ammonia measurement in Helicobacter pylori infection. Digestive diseases and sciences 2002;47:2523-30. 



\section{Chapter}

\section{Impact of hernia volume on pulmonary complications following complex hernia repair}

Elwin H.H. Mommers ${ }^{1,2}$, Johannes A. Wegdam ${ }^{1}$, Sander van der Wolk ${ }^{3}$, Simon W. Nienhuijs ${ }^{4}$, Tammo S. de Vries Reilingh ${ }^{1}$

${ }^{1}$ Department of Surgery, Elkerliek Hospital, Helmond, The Netherlands ${ }^{2}$ Department of Surgery, Maastricht University Medical Center, Maastricht, The Netherlands

${ }^{3}$ Department of Radiology, Elkerliek Hospital, Helmond, The Netherlands

${ }^{4}$ Department of Surgery, Catharina Hospital, Eindhoven, The Netherlands

Journal of surgical research 2017 May;(211):8-13. doi:10.1016/j.jss.2016.11.051. Epub 2016 Dec $7^{\text {th }}$ 


\section{ABSTRACT}

Background: Despite a multitude of evidence-based prediction models and risk factors for postoperative complications after ventral hernia repair, estimating a patients' risk of postoperative complications after ventral hernia repair remains challenging. To improve the preoperative assessment of complex hernia patients, some studies have examined pulmonary changes after hernia repair hypothesizing that large hernias lead to pulmonary changes and increased pulmonary complication rates. Some studies have described a correlation between hernia volume and pulmonary changes, although none provided compelling evidence to identify hernia volume as a risk factor for pulmonary complications. This study evaluates the relationship between hernia volume and postoperative pulmonary complications using computed tomography (CT)-based volume measurements.

Methods: Analysis of a prospectively maintained database of consecutive complex hernia patients from 2011 to 2014 undergoing endoscopic (ECST) or open component separation technique (CST) for a hernia defect with a minimum width of $6 \mathrm{~cm}$ and visual protrusion of the hernia sac ventral of the rectus abdominis muscles in supine position was performed. Hernia volume was calculated using multiple plane reconstruction of a standard abdominal CT-scan. Noted endpoints were pulmonary complications.

Results: Thirty-five patients underwent ECST $(n=20)$ or CST $(n=15)$ with a median defect volume of $474 \mathrm{~cm} 3$ (range, 114-2086 cm3). Observed complications were pneumonia $(n=4)$, pulmonary infiltrate $(n=3)$, aspiration pneumonia $(n=2)$, and acute respiratory distress syndrome $(n=1)$. Univariate and multivariate analyses showed that pulmonary complications were associated with "hernia volume" ( $\mathrm{P}=0.045 ; 95 \% \mathrm{Cl}$ : 1.008-1.910).

Conclusions: Hernia volume is a promising risk factor for postoperative pulmonary complications and can be calculated using a standard abdominal CT-scan. 


\section{INTRODUCTION}

Reducing large ventral hernias is accompanied with a postoperative complication rate up to $57 \%$, depending on the technique, the patients' comorbidity and complexity of the hernia. ${ }^{1-7}$ Despite a multitude of evidence based prediction models and risk factors for postoperative complications, estimating a patients' risk of complications following ventral hernia repair remains challenging. ${ }^{8}$ Hernia repair centers use preoperative multidisciplinary assessments to evaluate patients and estimate their postoperative risk for complications. ${ }^{9}$ These assessments include variables such as Body Mass Index (BMI), percentage of glycosylated haemoglobin ( $\mathrm{HbA} 1 \mathrm{C})$, age, co-morbidity and smoking. ${ }^{9}$ In an attempt to improve the aforementioned assessment, several studies have analysed pulmonary changes after ventral hernia repair. ${ }^{10-12}$ These studies hypothesized that when a large ventral hernia is reduced inside the abdominal cavity the intra-abdominal volume will rise. Since the abdominal wall limits the abdomen in expanding outward, the intra-abdominal pressure will increase, causing pulmonary compliance to decline. ${ }^{12}$ Despite experts agreeing on the validity of this theory none of the previously mentioned studies directly evaluated the correlation between hernia volume and the risk of pulmonary complications. ${ }^{10,13}$ This study evaluates the relationship between hernia volume and pulmonary complications using CT-based volume measurements and a prospectively maintained database of pulmonary complications.

\section{METHODS}

During 2011 and 2014 all patient undergoing complex ventral hernia repair were registered at a hospital specialised in abdominal wall surgery. ${ }^{1,14}$ Patient characteristics, pulmonary function and complications were registered prospectively in an electronic ventral hernia database. Consecutive patients with ventral hernias were extracted from this database if they fulfilled the following criteria. Any patient with a midline hernia $\geq 6$ $\mathrm{cm}$ in width that underwent 'endoscopically assisted' (ECST) or 'open' components separation technique (CST) with evident protrusion of the hernia content ventral of the rectus abdominis muscles in a relaxed supine position. Exclusion criteria were any patient with rectus diastasis without a true ventral hernia, previous subcutaneous dissection, American Society of Anesthesiologists (ASA) classification IV or $\mathrm{V}^{15}$, Body Mass Index $(\mathrm{BMI})>30 \mathrm{~kg} / \mathrm{m}^{2}$ and patients with a reduced forced expiratory volume $(\leq 80 \%)$ and/or Vital capacity ( $\leq 70 \%)$ when compared to the predicted value. ${ }^{16,17}$

All patients underwent a standard preoperative abdominal CT-scan without Valsalva manoeuvre. All hernias were classified using the European Hernia Society (EHS) guidelines for hernia classification. ${ }^{18}$

The endoscopic component separation technique was performed as described by Mommers et al. ${ }^{14}$ All operations were performed by three experienced gastrointestinal 
surgeons trained in abdominal wall reconstructions (JW, SN and TdVR). Patients were followed at the outpatient clinic (2, and 6 weeks, 3, 6, 12, and 24 months postoperative). Recurrence was assessed during physical examination in standing and supine position, if a clinical recurrence was uncertain an abdominal CT-scan was performed.

\section{Volume measurements}

Hernia volume was expressed as a percentage of the total abdominal volume (hernia sac volume (HSV) + abdominal cavity volume (ACV)) that resided inside the hernia sac during standard abdominal CT-scan without Valsalva maneuver, and calculated by dividing HSV through the total abdominal volume. HSV was defined as the volume in front of the ACV, demarcated by the parieto-peritoneum of the hernia sac.

All volumes were measured using volume analysis software (ViewForum R6.3V1L3 version 2008) from Philips Healthcare / Philips Medical System (figure 1). Outlining of the volume was done partially by hand using the 'contour stack' method to calculate volume within a Multiple Plane Reconstruction of a standard abdominal CT-scan. A slice thickness of $2 \mathrm{~mm}$ was used, after every 4-5 slices a new outlining was made by hand. The software interpolated the contours between the outlined areas based on Hounsfield units of the outlined areas. All measurements were performed by the same author (EM) blinded for patient outcome.

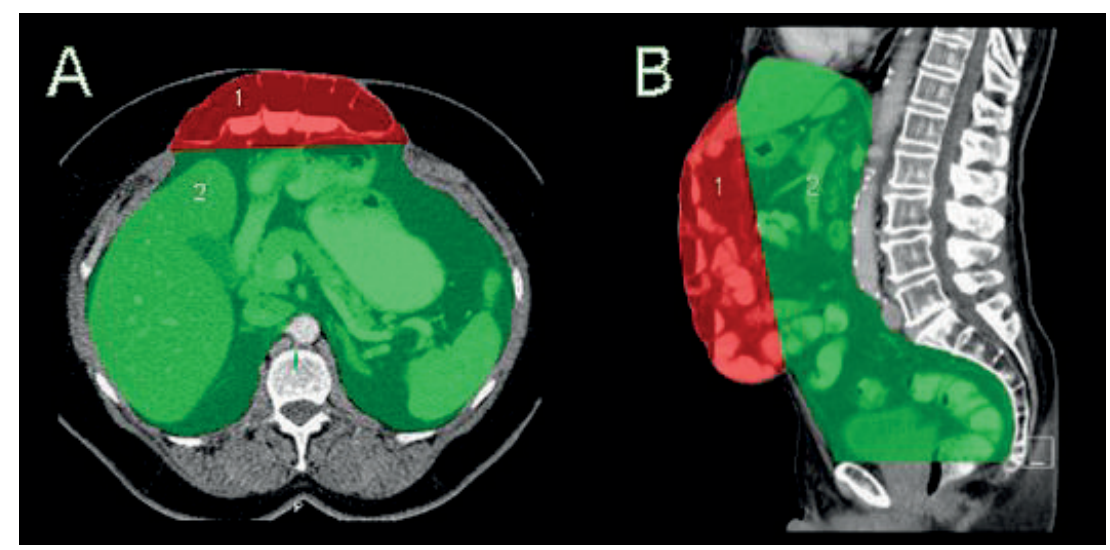

Figure 1 CT-outlining of hernia sac volume (HSV) and abdominal cavity volume (ACV)

A: transversal slide showing CT-outlined hernia volume (1 red) and intraperitoneal space (2 green). $\underline{B}$ : Sagittal slide showing hernia volume and abdominal cavity volume with caudal limit over the pubic symphysis.

\section{Statistical analysis}

Univariate and multivariate analysis were performed to identify statistically significant risk factors for postoperative pulmonary complications. Only known confounders or 
variables that showed a statistical significance of $\leq 0.06$ (approaching alfa of 0.05 ) in the univariate analysis were included in a multivariate logistic regression analysis to correct for any interactions between the variables. Statistical analysis was performed with IBM crop. SPSS statistics for windows', version 24, released 2014, Armonk, NY: IBM corp. Correlations were calculated using Pearsons R, Regression analysis was performed using binomial multivariate logistic regression analysis, standard distribution was tested with the Shapiro-Wilk test, Wilcoxon Signed Ranks test or paired two-sided students T-test was used to compare means depending on the probability distribution of the included variables.

\section{RESULTS}

Thirty-five patients (13 female, 22 male) with a median age of 63 years (range 39-77), mean body mass index (BMI) of $27 \mathrm{~kg} / \mathrm{m}^{2}\left(\mathrm{SD} \pm 6.4 \mathrm{~kg} / \mathrm{m}^{2}\right)$ and median American Society of Anesthesiologists classification (ASA) 2 (range 1-3) were included (table 1). All but three patients received mesh augmentation. The median hernia volume was $5.4 \%$ (range 1-25\%) with an absolute volume of $474 \mathrm{~cm}^{3}$ (range $114-2086 \mathrm{~cm}^{3}$ ). Two patients had chronic obstructive pulmonary disease (COPD) Gold II and one patient had asthma. All patients fulfilled the criteria for complex hernia as formulated by Slater et al., 'minor' $^{\prime}(n=11)$, 'moderate' $(n=18)$, 'major' $(n=6) .{ }^{19}$

\section{Postoperative complications}

Seven patients experienced seven mild pulmonary complications, and three patients experienced severe postoperative pulmonary complications. Two of these patients died, one due to sepsis caused by aspiration pneumonia and one due to multiple organ failure and acute respiratory distress syndrome (table 2). Median hospital stay was 6 days (range 3-103 days).

Table 1 Demographic, perioperative, and recurrence details

\begin{tabular}{ll}
\hline Demographic characteristics $(\mathrm{n}=35)$ & \\
Age $(\mathrm{yr})$, median (range) & $64(39-77)$ \\
Gender (male/female) & $22 / 13$ \\
BMI $\left(\mathrm{kg} / \mathrm{m}^{2}\right)$ mean (SD) & $26.7( \pm 3.4)$ \\
Smoking & $6(17 \%)$ \\
Ex-smoker & $10(29 \%)$ \\
Diabetes, Type 1 Type 2 & $1(2 \%) / 6(17 \%)$ \\
ASA classification III & $10(29 \%)$ \\
Previous repair & $11(31 \%)$ \\
Abnormal Preoperative PFT & $5(14 \%)$ \\
\hline
\end{tabular}




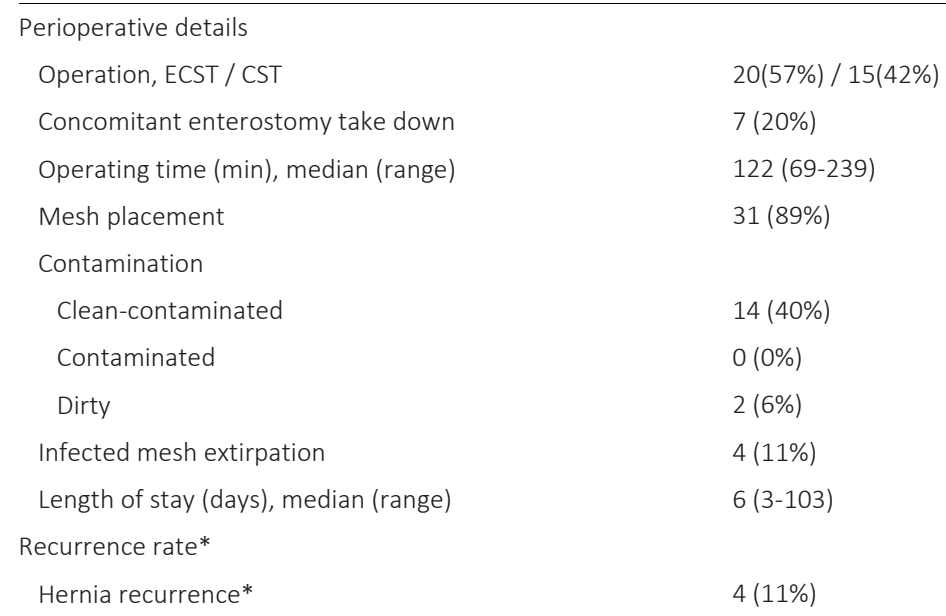

Demographic characteristics of all included patients. (\%); percentage of total population, SD; Standard deviation, ASA; American Society of Anesthesiologists classification, PFT; Pulmonary function test, (E)CST; (endoscopic) components separation technique, contamination; Clavien Dindo classification for surgical complications, Smoking; occasional smoker, Ex-smoker: stop smoking $\geq 3$ months before surgery.

Table 2 Pulmonary complications $\leq 30$ days postoperative

\begin{tabular}{ll}
\hline Complications Class I or II $(n=8)$ & $4(11 \%)$ \\
Pneumonia & $4(11 \%)$ \\
Pulmonary infiltrate & \\
Complications Class III, IV or V ${ }^{\#}(n=3)$ & $2(6 \%)$ \\
Aspiration pneumonia & $1(3 \%)$ \\
Acute respiratory distress syndrome & \\
\hline
\end{tabular}

${ }^{\#}$ Clavien Dindo classification for surgical complications ${ }^{20}$; Pneumonia was defined as the presence of a pulmonary infiltrate on chest radiograph in combination with fever or a positive sputum culture; pulmonary infiltrate was defined as an infiltrate seen on chest radiograph without the presence of fever or a positive sputum culture.

\section{Statistical analysis}

A univariate analysis was performed to identify the influence of a multitude of risk factors on postoperative pulmonary complications (table 3). Hernia volume was the only statistically significant predictor $(p=0.018)$ for pulmonary complications. The variable 'operation Method' ( $p=0.056)$, did not show a statistically significant effect in the univariate model, though since this dataset included two types of component separation techniques (ECST and CST), each associated with different degrees of release and risk of pulmonary complications the 'operation method' variable was identified as a confounder in this dataset. Therefore, it was entered in the multivariate model despite the lack of statistical significance (table 3). Additionally, the risk factor 'smoking' showed a near statistical significant result $(p=0.059)$. These three variables were entered in a binomial 
multivariate logistic regression analysis model to correct for interaction and confounding. The logistic regression model explained $44 \%$ (Nagelkerke $\mathrm{R}^{2}$ ) of the variance in the occurrence in postoperative pulmonary complications and correctly classified $77 \%$ of patients with significant goodness of fit (Hosmer and Lemeshow test $X^{2}=7.858$; $\mathrm{p}=0.345$ ). In the multivariate model 'hernia volume' was the only statistically significant variable. Based on the Wald $X^{2}$ test values in the uni- and multivariate models, 'hernia volume' was the most influential variable followed by 'smoking' and 'operation method'. A receiver operating characteristics (ROC) curve analysis (area under the curve 0.772 ) showed $5.6 \%$ evisceration as optimal cut-of value for increased risk of pulmonary complications (sensitivity 0.80 , specificity 0.68 ).

\section{DISCUSSION}

The concept of a correlation between hernia volume and postoperative pulmonary complications has been suggested previously, although compelling evidence for a correlation between hernia volume and pulmonary complications was not provided up till now. ${ }^{10,13,19,21}$ Using multivariate analysis, we were able to provide the first statistically significant evidence for a relationship between pulmonary complications and hernia volume. Gaidukov and colleagues proved that the intra-abdominal pressure rises after ventral hernia repair and a subsequent reduction in pulmonary dynamic compliance of $15-20 \%$ occurs. ${ }^{12}$ Sabbagh et al. investigated the relationship between hernia volume and abdominal compartment syndrome in seventeen patients with giant incisional ventral hernia that were treated with preoperative progressive pneumo-peritoneum. ${ }^{10}$ They divided their population in two groups based on the need for additional interventions to close the abdomen (i.e. colonic resection) and observed that the group with additional interventions had a higher loss-of-domain. They used a cut-off value of $20 \%$ as predictor for tension free-closure in a multivariate logistic regression analysis (Odd's $35 ; 95 \% \mathrm{Cl}$ [1.38-888] $\mathrm{p}<0.05)$. Agnew et al. investigated diaphragm height and changes in pulmonary function tests after a component separation technique and found no significant difference. ${ }^{11}$ Considering the study by Gaidukov et al, diaphragm height may not be the most sensitive tool to assess pulmonary changes after hernia repair. Agnew et al. measured pulmonary function and diaphragm height three months after hernia repair. Arguably, this period is too long to detect pulmonary changes that occurred due to the operation, since any change in diaphragm height may have been compensated during the first three postoperative months.

Our study uses the observations of Sabbagh et al. and Gaidukov et al. to explore any correlation between hernia volume and pulmonary complications using CT-based volume calculation combined with uni- and multivariate statistical analysis. A statistically significant relationship between hernia size and pulmonary complications was found in both the univariate and multivariate logistic regression models. Based on these models 
an increase in evisceration of $1 \%$ would increase the odds of getting a pulmonary complication with 1.387 . The ROC-curve analysis showed $5.6 \%$ evisceration as optimal predictive value for pulmonary complications. In our series, with a median abdominal content of $9177 \mathrm{cc}$, this would indicate that any patient with a hernia volume larger than 514 cc on standard abdominal CT-scan without Valsalva manoeuvre has an increased risk of pulmonary complications. However, given the small sample size and the number of mild complications included in this study this value could be underestimated.

The software used in this study to calculate hernia volume can import any standard abdominal CT-scan, without the need for additional scanning protocols or sequences. Measuring the abdominal and hernia volume will take approximately 15-20 minutes per patient. Therefore, performing this measurement will not lead to additional costs, other than the invested time. The authors would like to stipulate that hernia volume measurement should be performed using exact CT-based measurement software, such as used in this study. Measuring volume with mathematical formulas such as described by Tanaka et al. is deemed unfit since these methods are not validated for exact volume calculation. $^{22}$

This study provides the first preliminary evidence for the existence of a direct correlation between hernia volume and pulmonary complications. Though there are limitations that must be accentuated. The sample size is small and therefore the number of events in the multivariate model is low, increasing the risk of bias. Due to the small population ROC-curve analysis only provides a crude estimation of the optimal cut-off value. Therefore, the results of this study must be interpreted with caution. In addition, consecutive patients were derived from a prospectively registered database of complex hernia patients, resulting in a population with pulmonary comorbidities and increased risk for pulmonary events. Ideally a population without pulmonary comorbidity should be used to isolate the effect of hernia volume more accurately. However, patients with large ventral hernias tend to have a high number of comorbidities, making patients without risk factors for pulmonary complication rare. Despite the previously mentioned limitations, the current study demonstrates the importance of hernia volume as a risk factor for complications in a high-risk population and encourages future research in preoperative risk assessments using hernia volume.

\section{Recommendations for future research}

Hernia volume can be expressed in different manners. Using an absolute volume for statistical analysis will not result in a reliable outcome. Since the total abdominal volume can vary greatly between patients, hernia volume must be expressed as a ratio or percentage of the total volume to create a comparable factor between different patients. Moreover, different limits can be used to define the intra-abdominal volume (i.e. with or without retroperitoneal organs). For future research, the authors would rec- 
ommend the anatomical boundaries of volume measurement stated by Sabbagh et al. which were also applied in this study.

\section{CONCLUSION}

Hernia volume is a promising risk factor for postoperative complications and can be calculated using standard abdominal CT-scans. To evaluate the effect of hernia volume in more detail, we recommend a larger study with more statistical power to isolate the impact of hernia volume on pulmonary complications more accurately.

\section{ACKNOWLEDGMENTS}

The authors thank G. Clappers-Gielen, C. Jacobs and P. Matheï for evaluating all patients during the inclusion period, the multi-disciplinary team meetings, and post-operative care. 


\section{REFERENCES}

1. de Vries Reilingh TS, van Goor H, Rosman C, et al. "Components separation technique" for the repair of large abdominal wall hernias. J Am Coll Surg 2003;196:32-7.

2. Giurgius M, Bendure L, Davenport DL, Roth JS. The endoscopic component separation technique for hernia repair results in reduced morbidity compared to the open component separation technique. Hernia 2012;16:47-51. doi: 10.1007/s10029-011-0866-1. Epub 2011 Aug 11.

3. Harth KC, Rose J, Delaney CP, Blatnik JA, Halaweish I, Rosen MJ. Open versus endoscopic component separation: a cost comparison. Surg Endosc 2011;25:2865-70.

4. Harth KC, Rosen MJ. Endoscopic versus open component separation in complex abdominal wall reconstruction. Am J Surg 2010;199:342-6; discussion 6-7.

5. Ghali S, Turza KC, Baumann DP, Butler CE. Minimally invasive component separation results in fewer wound-healing complications than open component separation for large ventral hernia repairs. J Am Coll Surg 2012;214:981-9.

6. Ramirez OM, Ruas E, Dellon AL. "Components separation" method for closure of abdominal-wall defects: an anatomic and clinical study. Plast Reconstr Surg 1990;86:519-26.

7. Mudge M, Hughes LE. Incisional hernia: a 10 year prospective study of incidence and attitudes. Br J Surg 1985;72:70-1.

8. Mitchell TO, Holihan JL, Askenasy EP, et al. Do risk calculators accurately predict surgical site occurrences? J Surg Res 2016;203:56-63. doi: 10.1016/j.jss.2016.03.040. Epub Mar 26.

9. Liang MK, Holihan JL, Itani K, et al. Ventral Hernia Management: Expert Consensus Guided by Systematic Review. Annals of surgery 2016.

10. Sabbagh C, Dumont F, Robert B, Badaoui R, Verhaeghe P, Regimbeau JM. Peritoneal volume is predictive of tension-free fascia closure of large incisional hernias with loss of domain: a prospective study. Hernia 2011;15:559-65.

11. Agnew SP, Small W, Jr., Wang E, Smith LJ, Hadad I, Dumanian GA. Prospective measurements of intraabdominal volume and pulmonary function after repair of massive ventral hernias with the components separation technique. Annals of surgery 2010;251:981-8.

12. Gaidukov KM, Raibuzhis EN, Hussain A, et al. Effect of intra-abdominal pressure on respiratory function in patients undergoing ventral hernia repair. World J Crit Care Med 2013;2:9-16.

13. Yao S, Li JY, Liu FD, Pei LJ. Significance of measurements of herniary area and volume and abdominal cavity volume in the treatment of incisional hernia: application of CT 3D reconstruction in 17 cases. Comput Aided Surg 2012;17:40-5.

14. Mommers EH, Wegdam JA, Nienhuijs SW, de Vries Reilingh TS. How to perform the endoscopically assisted components separation technique (ECST) for large ventral hernia repair. Hernia 2016;20:441-7.

15. ASA Physical Status Classification System. 2014. 01-10-2014, at https://www.asahq.org/clinical/physical status.htm.)

16. Falagas ME, Kompoti M. Obesity and infection. Lancet Infect Dis 2006;6:438-46.

17. Ashburn DD, DeAntonio A, Reed MJ. Pulmonary system and obesity. Crit Care Clin 2010;26:597-602.

18. Muysoms FE, Miserez M, Berrevoet F, et al. Classification of primary and incisional abdominal wall hernias. Hernia 2009;13:407-14. doi: 10.1007/s10029-009-0518-x. Epub 2009 Jun 3.

19. Slater NJ, Montgomery A, Berrevoet F, et al. Criteria for definition of a complex abdominal wall hernia. Hernia 2014;18:7-17.

20. Clavien PA, Barkun J, de Oliveira ML, et al. The Clavien-Dindo classification of surgical complications: fiveyear experience. Ann Surg 2009;250:187-96. doi: 10.1097/SLA.0b013e3181b13ca2.

21. Rohrich RJ, Lowe JB, Hackney FL, Bowman JL, Hobar PC. An algorithm for abdominal wall reconstruction. Plast Reconstr Surg 2000;105:202-16; quiz 17.

22. Tanaka EY, Yoo JH, Rodrigues AJ, Utiyama EM, Birolini D, Rasslan S. A computerized tomography scan method for calculating the hernia sac and abdominal cavity volume in complex large incisional hernia with loss of domain. Hernia 2010;14:63-9. 


\section{PART}

INTRAOPERATIVE 



\title{
Chapter
}

\section{How to perform the endoscopically assisted components separation technique (ECST) for large ventral hernia repair}

\author{
Elwin H.H. Mommers ${ }^{1,2}$, Johannes A. Wegdam ${ }^{1}$, Simon W. Nienhuijs ${ }^{2}$, \\ Tammo S. de Vries Reilingh ${ }^{1}$ \\ ${ }^{1}$ Department of Surgery, Elkerliek Hospital, Helmond, The Netherlands \\ ${ }^{2}$ Department of Surgery, Catharina Hospital, Eindhoven, The Netherlands
}

Hernia 2016 Jun;20(3):441-7. doi:10.1007/s10029-016-1485-7. Epub 2016 Apr $1^{\text {st }}$ 


\section{ABSTRACT}

Background: The components separation technique (CST) is frequently used for reconstructing large ventral hernias. Unfortunately, it is associated with a high wound complication rate up to $50 \%$, caused by large wound surface and inherent trauma to abdominal skin vascularization. An endoscopically assisted modification of the original technique (ECST) spares skin vascularization and reduces wound surface, supposedly reducing wound complications. This study accurately describes ECST step by step with detailed illustrations and report the results of a 27-patient cohort.

Methods: Since September 2012 patients with midline hernias without previous subcutaneous dissection and a maximum diameter of approximately 10-15 cm underwent ECST in an expert center for abdominal wall reconstructions. Prospective data was gathered during inpatient care and 3-6 monthly follow-up.

Results: Twenty-seven patients (17 male; 10 female) with median age of 60 years (range 35-77), average BMI 27 (SD \pm 2 ) kg/m² and median ASA classification 2 (range 1-3) underwent ECST. Two patients were excluded due to bilateral conversion to conventional CST and finding of peritoneal metastases. Median defect size was $116 \pm 48 \mathrm{~cm} 2$. Median length of stay was 5 days (range 3-15). Wound complication rate was $11 \%$. Recurrence rate was $29 \%$ after a median follow-up of 13 months.

Conclusions: Endoscopically assisted modification of the original technique can be used for reconstructing large and complex ventral hernias up to $15 \mathrm{~cm}$ in diameter. The results of this small sized cohort study showed that ECST is feasible in patients with a uro-, or enterostomy and suggest that ECST reduces wound complication rate when compared to CST. 


\section{INTRODUCTION}

Over $20 \%$ of midline laparotomies causes an incisional hernia within 10 years. ${ }^{1,2}$ Repairing large ventral hernias is challenging in terms of operation technique, complications, and durability of the repair. In 1990 Ramirez et al. described the 'components separation technique' (CST) as a method for large ventral abdominal wall reconstruction. ${ }^{3}$ This technique is based on enlargement of the abdominal wall surface by transection of the external oblique muscle to create a compound flap of the internal oblique and transverse muscle. CST can be used to close hernias up to $20 \mathrm{~cm}$ width, however, due to the large wound surface patients are prone for post-operative wound complications (52\% wound complications rate). In an attempt to reduce post-operative wound complications, an endoscopically assisted version of the component separation technique (ECST) was developed. ECST reduces the wound surface and spares the innervations and blood supply of the abdominal skin. ECST seems to be associated with less wound complications when compared to CST. ${ }^{4-14}$ The present study describes the Endoscopically assisted Components Separation Technique and reports the results of a 27 patient cohort. ${ }^{15}$

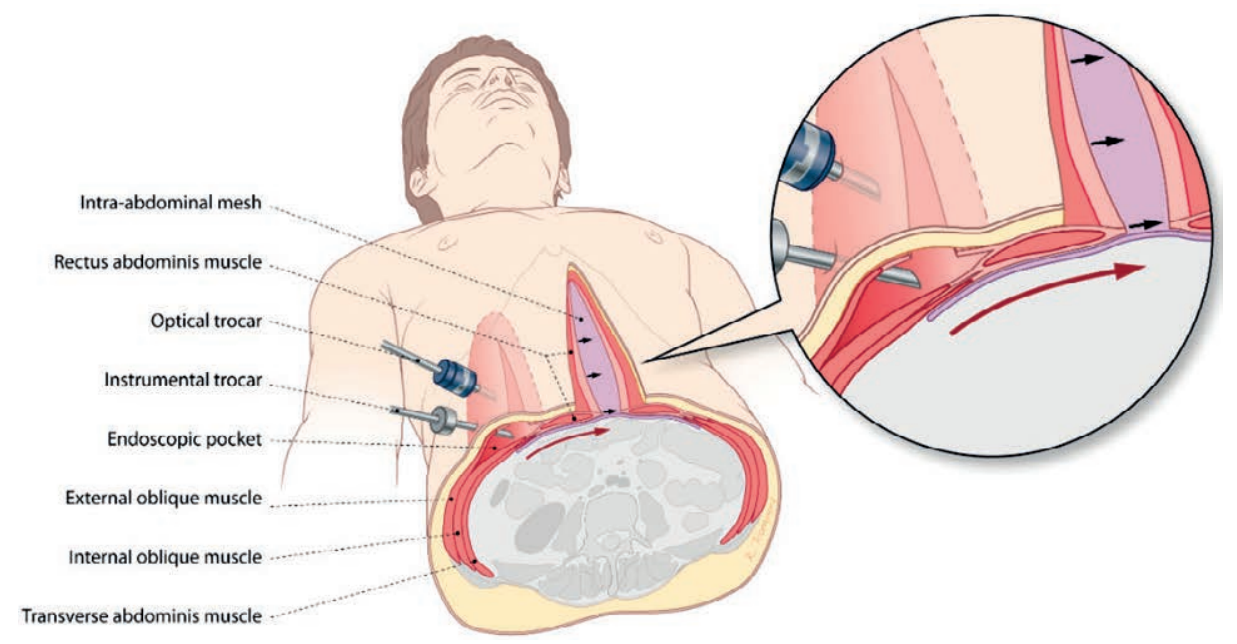

Figure 1 Birds eye view of ECST procedure

Overview of anatomy and trocar placement during ECST. Trocars are placed as far lateral as possible, the endoscopic pocket ranges from the thorax $\left(5 \mathrm{~cm}\right.$ cranial of the $12^{\text {th }}$ rib) until the inguinal ligament. Release of the external oblique aponeurosis creates a compound flap of the rectus abdominis muscle, the internal oblique and transverse muscles that can be advanced towards the midline approximately $6 \mathrm{~cm}$ at the umbilical level. 


\section{PERFORMING ECST}

\section{Step 1 'Patient preparation'}

All patients received preoperative intravenous antibiotic prophylaxis in the form of Cefazoline (Kefzol $\left.{ }^{\circ}\right) 1000 \mathrm{mg}$ and Metronidazol (Flagyl $\left.{ }^{\circ}\right) 500 \mathrm{mg}$. The patient is placed in a supine position, a peripheral intravenous line is placed on either arm. Both arms are tucked in alongside the trunk using a cotton sheet. A Foley Catheter is placed in the bladder and the skin is disinfected from the thorax to the pubic area with $2 \%$ chlorhexidine in $70 \%$ alcohol. The sterile field is created using sterile drapings and extends from the thorax to the pubic area and as far lateral as possible.

\section{Step 2 'Access the abdomen \& perform adhesiolysis'}

The midline scar is excised and the hernia is reduced. The hernia sac is removed and any adhesions between the intra-abdominal content and the abdominal wall are removed as far lateral as possible. The falciform ligament is dissected for optimal positioning in case an intra-abdominal mesh placement is used later. In case a sublay mesh placement is performed the falciform ligament can stay intact.

\section{Step 3 'Create the lateral endoscopic pockets'}

See figure 1 and 2 for optimal trocar placement and position of the 'endoscopic pocket'. A small $1.5 \mathrm{~cm}$ incision is made $2 \mathrm{~cm}$ subcostal, approximately $5 \mathrm{~cm}$ lateral from the rectus muscle. Through this incision the fascia of the external oblique muscle is identified and incised. The muscle fibers are spread carefully with two blunt handheld retractors and a blunt tip balloon trocar (10 mm AutoSuture BTT, Covidien ${ }^{\circledR}$ ) is placed in the plane between the external and internal oblique muscle. Insufflation commences, a 10 $\mathrm{mm} 30^{\circ}$ endoscope is introduced and used to separate the loose connective tissue between the external and internal oblique muscle, thus creating the 'endoscopic pocket'. One $5 \mathrm{~mm}$ screw-type trocar is placed under direct vision $3 \mathrm{~cm}$ distal to the blunt-tip trocar. Using electro coagulation, the pocket is extended cranially more than $5 \mathrm{~cm}$ above the costal margin, distally towards the inguinal ligament and laterally to the aponeurosis of the external oblique muscle (figure 1). The external oblique aponeurosis is now released approximately $1 \mathrm{~cm}$ lateral to the rectus abdominal muscle more than $5 \mathrm{~cm}$ cranial of the costal margin until the inguinal region to create a compound flap consisting of the internal oblique and transverse muscle. See figure 3 for an endoscopic view inside the endoscopic pocket after the external oblique muscle has been transacted. The same procedure is performed on the contralateral side. 

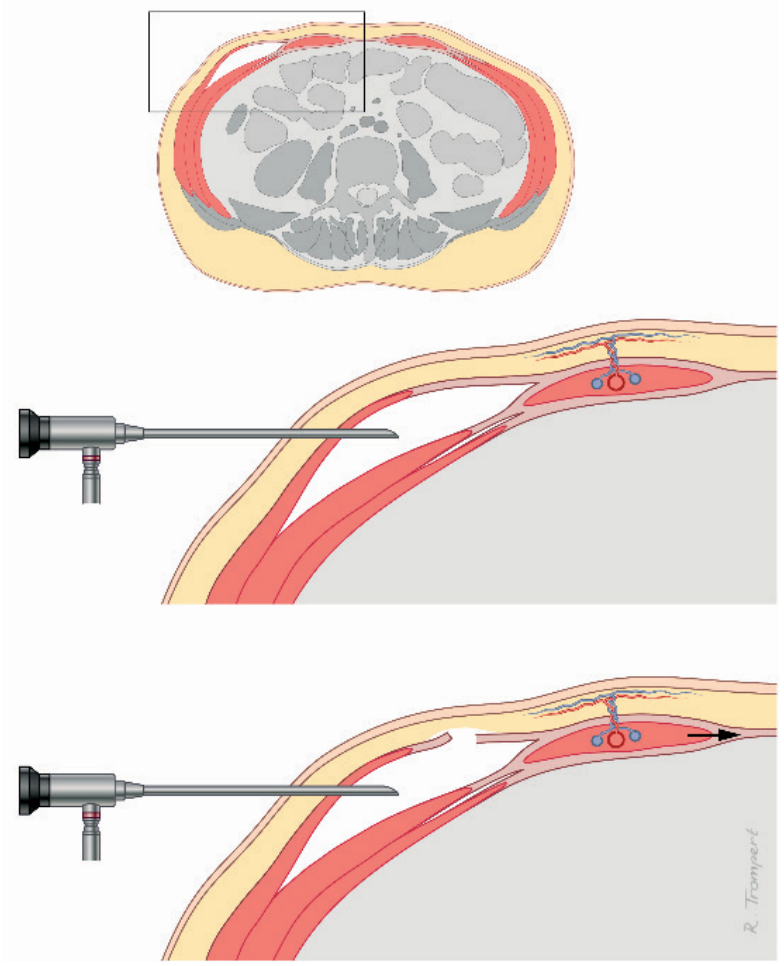

Figure 2 Transverse view before and after release of the external oblique muscle.

Transverse view of the abdomen visualizing the endoscopic pocket and the release of the external oblique aponeurosis. Note that the perforating vessels and nerves are spared.

\section{Step 4 'Close the gap'}

The newly created compound flap consisting of the rectus muscle, internal oblique muscle, and transverse abdominal muscle (see enlargement in figure 1) can now be advanced approximately $6 \mathrm{~cm}$ towards the midline on either side. Closing gaps between 10 and $15 \mathrm{~cm}$ in width. The trocars in the endoscopic pockets can now be removed and the external oblique fascia is closed with a single resorbable suture.

Mesh reinforcement is performed using one of two mesh positions: 'intraabdominal' or 'sublay'. Indication for an intra-abdominal mesh exists when the posterior rectus fascia can't be closed in the midline or when the posterior rectus fascia is of poor quality for instance due to previous surgery/scarring of the posterior rectus fascia.

If an intra-abdominal mesh is placed (Ventralight ST mesh Bard Davol inc. ${ }^{\circ}$ of 33×25 $\mathrm{cm}$ ) it is placed directly on the omentum and should cover the entire ventral exposure of the intra-abdominal compartment, ranging from xyfoid to pubic bone with a width of at least $25 \mathrm{~cm}$. The mesh then fixated using approximately ten transfascial polydioxanone sutures $(2 / 0)$ placed through the transverse abdominal and internal oblique muscle. 
In case the posterior rectus abdominis fascia is of good quality and can be closed in the midline, a sublay position of the mesh (underneath the rectus abdominis muscle, on top of the posterior rectus fascia) is preferred due to the reduced risk of bowel adhesions. For sublay placement the mesh (Bard Softmesh ${ }^{\circledR}$ Bard Davol inc. ${ }^{\circledR}$ of $30 \times 30 \mathrm{~cm}$ ) was cut to size to cover the entire posterior rectus abdominis fascia and fixated using at either end using four absorbable sutures. The anterior rectus abdominis fascia is closed using a continues resorbable PDS loop suture. The skin is closed and the wounds are dressed. The patient is advised to wear an abdominal binder for four weeks and avoid heavy lifting for six weeks.

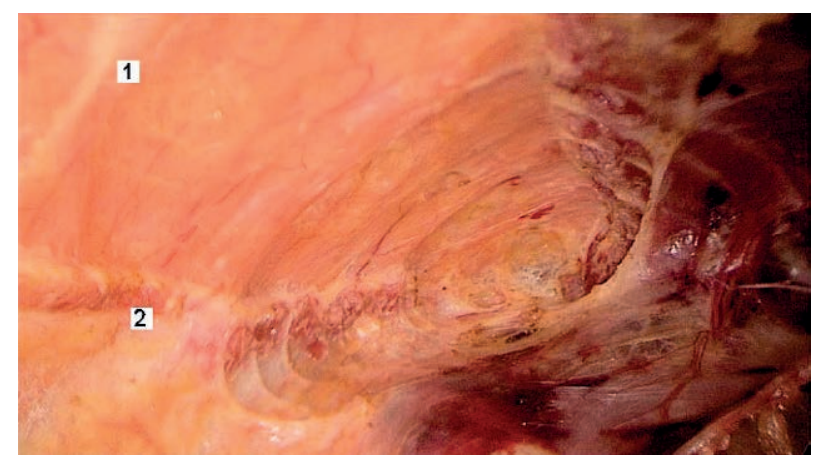

Figure 3 Endoscopic view after release of the external oblique muscle.

Endoscopic view of the 'pocket' after release of the external oblique aponeurosis. The subcutaneous fat (1) is visible as well as the medial rim of the external oblique aponeurosis (2).

\section{TIPS AND TRICKS}

\section{Tip 1 'Patient selection'}

ECST can be used for midline hernias approximately six to thirteen $\mathrm{cm}$ wide. In patient selection it is of the utmost importance that the patient is in good pulmonary condition. Ideally a multi-disciplinary approach including pulmonary screening, radiological examination using abdominal CT-scans and pre- and postoperative physical therapy are used to provide the best patient care. Patients with chronic obstructive pulmonary disease (COPD; Tiffenau index $<70 \%$ ) should receive perioperative pulmonary preparation (salbutamol and ipratropium) to prevent exacerbation, and patients with asthma should receive preoperative preparation with salbutamol to prevent bronchospastic reaction during induction. Patients with severe obesity should be stimulated to lose weight under supervision of a physical therapist, ideally the Body Mass Index (BMI) should be below $30 \mathrm{~kg} / \mathrm{m}^{2}$ before surgery. 


\section{Tip 2 'Presence of a uro-or enterostomy'}

The presence of a uro- or enterostomy limits the surgical techniques that can be used for ventral hernia repair. However, ECST can be used in these patients if a 'sublay' position of the mesh is feasible. Therefore, pre-operative evaluation should include a detailed examination of the patient history, including any previous hernia repairs and mesh positions and an abdominal CT-scan to provide inside in the quality of the abdominal muscles and the posterior rectus abdominis fascia. In step 3 of the procedure a sublay mesh position is used. After closing the posterior rectus fascia, the mesh is cut to size to cover the entire posterior rectus fascia, including the 'hole' in the posterior rectus fascia where the uro- or enterostomy is located. Then, the mesh is incised at the location of the stoma and a hole is cut inside the mesh to facilitate the uro-/enterostomy. The mesh is then placed on top of the posterior rectus abdominis fascia and resembles a 'keyhole' mesh around the uro-/enterostomy. Ideally the incision in the mesh used to cut the whole and place the mesh around the uro-/enterostomy is closed using non-absorbable sutures, however, depending on the size and location of the uro-/ enterostomy this is not always possible.

\section{Tip 3 'Monitor position'}

During ECST orientation inside the endoscopic pocket may be difficult for the performing surgeon. To assist the surgeon with orientation during the endoscopic release two endoscopic video monitors are used, one at the head of the operating table and one at the end of the operating table. If the surgeon points the endoscope towards the head of the patient, the monitor located at that side of the table is used and vice versa of the endoscope is pointed towards the feet of the patient.

\section{Tip 4 'Patient position'}

To ensure enough room for manipulation of the endoscope during the creation of the endoscopic pocket both arms are tucked in alongside the trunk of the patient and trocars must be placed far laterally.

\section{Tip 5 'Creating the endoscopic pocket'}

The 'space' or 'pocket' between the external and internal oblique muscle may be difficult to identify due to its resemblance to the 'space' between the transverse and the internal oblique muscle. However, after placement of the balloon trocar and insufflation of the 'endoscopic pocket' the surgeon can quickly check if he is creating the endoscopic pocket between the external and internal oblique muscle or (by mistake) in between the internal oblique and the transverse abdominal muscle since the first is an avascular 
plane and shouldn't be accompanied by any bleeding during dissection of the connective tissue and the later isn't. One may also use the direction of the muscle fibers in the 'ceiling' of the endoscopic pocket to identify either the external or the internal oblique muscle.

\section{Tip 6 'Use mesh'}

Previous studies have demonstrated that the use a mesh is vital to create a durable hernia repair. ${ }^{16}$ The results section below will also demonstrate that three patients who underwent a repair without mesh (two patients due to intra-abdominal contamination and one because of pain and discomfort after previous mesh repair) all had a recurrence hernia within two years.

\section{METHODS \& RESULTS}

During September 2012 and September 2015 twenty-seven patients (17 male, 10 female) with a median age of 60 years (range 35 to 77 years), average BMI of $27 \pm 2 \mathrm{~kg} / \mathrm{m}^{2}$ and median ASA 2 underwent ECST for correction of a midline incisional hernia with a median defect size of $116 \mathrm{~cm}^{2}$ (range 28 to 298, Table 1). Only patients with no previous subcutaneous dissection and a maximum defect diameter of approximately $10-15 \mathrm{~cm}$ were included.

Two patients had both a midline defect (EHS M3) and a parastomal hernia (EHS L2). All patients fulfilled the criteria for 'Minor' ( $n=9)$, 'moderate' $(n=14)$ or 'Major' $(n=4)$ complex hernias as formulated by Slater et al. ${ }^{17}$ Follow-up was obtained from 3-6 monthly outpatient visits.

\section{Postoperative complications}

Overall seven patients (26\%) had 10 mild (Clavien Dindo class. I or II) complications within 30 days postoperative (paralytic ileus with gastric paralysis $(n=2)$, urinary tract infection ( $n=2)$, pneumonia $(n=1)$, deep venous thrombosis $(n=1)$ ) and 1 class III complication (subcutaneous haematoma, normalized ratio (INR) $\geq 7.5$, in patient using Phenprocoumon).

Three of the previous seven patients (11\%) had 4 wound complications (wound dehiscence $(n=1)$, abscess $(n=3))$ all treated with negative pressure wound therapy. These three patients all had an increased risk of infection due to perioperative intraabdominal contamination (dirty $(n=1)$, clean-contaminated $(n=2)){ }^{18}$ 
Table 1 Demographic and perioperative details ( $n=27)$

\begin{tabular}{|c|c|}
\hline Demographic characteristics & \\
\hline Age (yr), median (range) & $60(35-77)$ \\
\hline Gender (male/female) & $17 / 10$ \\
\hline $\mathrm{BMI}\left(\mathrm{kg} / \mathrm{m}^{2}\right)$ mean $(\mathrm{SD})$ & $27(2)$ \\
\hline Smoking & $5(19 \%)$ \\
\hline Ex-smoker & $6(22 \%)$ \\
\hline Previous repair & $9(33 \%)$ \\
\hline ASA classification III or IV & $2(7 \%)$ \\
\hline Diabetes & $2(7 \%)$ \\
\hline Abnormal preoperative PFT & $3(11 \%)$ \\
\hline Recurrence hernia & $8(29 \%)$ \\
\hline Previous incarceration of hernia & $1(4 \%)$ \\
\hline Location of hernia (M2, M3, M4) & $9,17,1$ \\
\hline Presence of urostomy, colostomy or ileostomy ${ }^{a}$ & $4(15 \%)$ \\
\hline Hernia width (cm), median (range) & $10(6-20)$ \\
\hline \multicolumn{2}{|l|}{ Perioperative details } \\
\hline ECST unilateral/bilateral & $3 / 24$ \\
\hline Concomitant enterostomy take down & $1(4 \%)$ \\
\hline Operating time (min), median (range) & $110(69-239)$ \\
\hline Mesh placement & $24(89 \%)$ \\
\hline \multicolumn{2}{|l|}{ Contamination } \\
\hline Clean-contaminated & $3(11 \%)$ \\
\hline Contaminated & $0(0 \%)$ \\
\hline Dirty & $2(7 \%)$ \\
\hline Infected mesh extirpation & $2(7 \%)$ \\
\hline Length of stay (days), median (range) & $5(3-15)$ \\
\hline
\end{tabular}

Demographic characteristics of all included patients; (\%); percentage of total population, SD; Standard deviation, ASA; American Society of Anesthesiologists classification, PFT; Pulmonary function test, (E)CST; (endoscopic) components separation technique, Smoking; occasional smoker, Ex-smoker: stop smoking $\geq 3$ months before surgery. ${ }^{a}$ Four patients had a uro-/enterostomy at the beginning of the procedure. Two of these patients underwent concomitant enterostomy takedown during the ECST. Location of the hernia is reported according to the European Hernia Society (EHS) classification for hernias ( $M 2=$ epigastric, $M 3=$ umbilical, M4=below the umbilicus). Contamination was classified according to the CDC classification.

\section{Recurrence rate}

To assess recurrence rate only patients with a follow-up of one year or longer that received a repair with a mesh $(n=14)$ were evaluated. Evaluation was based on clinical examination, if any doubt of recurrence existed an ultrasound was performed $(n=2)$ to evaluate recurrence rate. After a median follow-up of 13 months (range 12 to 34) four of the fourteen patients that underwent ECST with mesh reinforcement had a recur- 
rence hernia after 12, 15,18 and 26 months respectively. Three of these four patients had only a minor, $3 \mathrm{~cm}$ recurrence in the epigastric region.

In addition to the above-mentioned recurrences, all patients that underwent a repair without mesh reinforcement $(n=3)$ had a recurrence hernia after 6,7 and 18 months respectively.

\section{DISCUSSION}

ECST can be used to reconstruct midline abdominal wall hernias up to approximately 15 $\mathrm{cm}$ in width, even in patients with a uro- or enterostomy.

\section{Operation technique}

Our technique differs from previously published methods such as performed by Lowe et al. ${ }^{13}$ It is the authors view that using a distension balloon to create a subcutaneous space has little advantage over the original technique described by Ramirez et al. because of trauma to the skin vascularization ${ }^{3}$. Rosen et al. and Fox et al. described an endoscopic method using the plane between the internal and external oblique muscles, though a dissection balloon is used, and the external oblique fascia is incised in three places because an extra trocar is needed to complete transection of the external oblique aponeurosis. ${ }^{7,19}$ In our series ECST was successfully used in hernias of 10-15 cm in width. The maximum diameter that was reconstructed with ECST in this series was $20 \mathrm{~cm}$ due to a larger than expected defect during the operation. Since this diameter was only successfully reconstructed once in our series the authors do not recommend using ECST routinely for defects over $15 \mathrm{~cm}$ in width.

\section{Wound complications}

Our prospectively collected data shows that ECST with mesh augmentation is associated with a modest wound complication rate of $11 \%$. These result, and the results from Albright et al., and Jensen et al., who reported wound complication rates of $19 \%$ and $18 \%$ respectively in the ECST group prove that ECST has a reduced wound complication rate when compared to CST . 4,9,15,20

Switzer and colleagues compared length of stay, wound complications, and recurrence rate in their meta-analysis between CST and ECST. ${ }^{15}$ Despite a clear tendency in favour of ECST in the descriptive data, they were unable to provide statistically significant evidence other than a reduction in operation time and skin dehiscence in favour of ECST. This lack of statistically significant results can most likely be attributed to a shortage of patients and stipulates the need for additional data. Jensen et al. provided the first statistically significant evidence for a reduction of wound complications with ECST 
in their meta-analysis of five comparative studies including a total of 162 patients (ECST $(n=78) 18 \%$ vs $43 \%(n=84)$ CST, $p<0,001)$. They could not find statistical evidence for a reduction in hernia recurrence or length of stay. However, the result of this metaanalysis must be interpreted with caution because the included studies are no randomized controlled trials and indication for open CST differed from ECST (prior mesh infection, contaminated procedures and extensive adhesiolysis) in most of the included studies, which increases the risk of selection bias in favour of ECST.

\section{Mesh repair}

The importance of a mesh in reducing the recurrence rate after CST was demonstrated by Slater et al. in their prospective study of 75 patients. ${ }^{16}$ They showed a recurrence rate of $38.7 \%$ in patients without a mesh and compared this with a literature extracted number of $14 \%$ recurrence in patients with a mesh $(p<0.01)$. In our series, we observed a $100 \%$ recurrence rate in all patients that underwent a non-mesh repair. Once again proving that mesh is a vital part of hernia reconstruction.

\section{Recurrence rate}

The series of Harth and Rosen used a comparable surgical technique with biological sublay or intra-peritoneal mesh. They described a high recurrence rate of $29 \%$ in both groups (ECST $n=22$ and CST $n=19$ ) and a wound complication rate of $27 \%$ and $52 \%$ in groups respectively, with a mean follow-up of 15 months. ${ }^{11,21}$ The results of Rosen et al. primarily describe a single stage treatment in contaminated cases (CST 91\% vs ECST $73 \%$ contaminated), reducing their external validity and comparability with a less complex population. Neither Jensen et al. nor Switzer at al. found a significant reduction of recurrence rate after ECST compared to CST in their meta-analysis. ${ }^{15,20}$ In our series, the recurrence rate is $29 \%$ after a median follow-up of 13 months, which suggests that ECST doesn't reduce the recurrence rate, however, due to a limited number of patients with a follow-up of 12 month or longer no conclusive statements can be made based on our series alone.

There are several limits to this study, such as the small number of patients, with a short follow-up. The analysis included results of both unilateral and bilateral ECST combined and did not included aesthetic outcome nor quality of life. Furthermore, no direct comparison to alternative hernia reconstruction methods were conducted.

In conclusion, the Endoscopically assisted Components Separation Technique can be used for reconstructing large and complex ventral hernias up to $15 \mathrm{~cm}$ in diameter. The results of this small sized cohort study showed that ECST is feasible in patients with a uro- or enterostomy and suggest that ECST reduces wound complication rate when compared to CST. 


\section{REFERENCES}

1. Fink $C$, Baumann $P$, Wente $M N$, et al. Incisional hernia rate 3 years after midline laparotomy. British Journal of Surgery 2014;101:51-4.

2. Mudge M, Hughes LE. Incisional hernia: a 10 year prospective study of incidence and attitudes. Br J Surg 1985;72:70-1.

3. Ramirez OM, Ruas E, Dellon AL. "Components separation" method for closure of abdominal-wall defects: an anatomic and clinical study. Plast Reconstr Surg 1990;86:519-26.

4. Albright E, Diaz D, Davenport D, Roth JS. The component separation technique for hernia repair: a comparison of open and endoscopic techniques. Am Surg 2011;77:839-43.

5. Butler CE, Campbell KT. Minimally invasive component separation with inlay bioprosthetic mesh (MICSIB) for complex abdominal wall reconstruction. Plast Reconstr Surg 2011;128:698-709.

6. Clarke JM. Incisional hernia repair by fascial component separation: results in 128 cases and evolution of technique. Am J Surg 2010;200:2-8.

7. Fox M, Cannon RM, Egger M, Spate K, Kehdy FJ. Laparoscopic component separation reduces postoperative wound complications but does not alter recurrence rates in complex hernia repairs. Am J Surg 2013;206:869-74; discussion 74-5.

8. Ghali S, Turza KC, Baumann DP, Butler CE. Minimally invasive component separation results in fewer wound-healing complications than open component separation for large ventral hernia repairs. J Am Coll Surg 2012;214:981-9.

9. Giurgius M, Bendure L, Davenport DL, Roth JS. The endoscopic component separation technique for hernia repair results in reduced morbidity compared to the open component separation technique. Hernia 2012;16:47-51. doi: 10.1007/s10029-011-0866-1. Epub 2011 Aug 11.

10. Harth KC, Rose J, Delaney CP, Blatnik JA, Halaweish I, Rosen MJ. Open versus endoscopic component separation: a cost comparison. Surg Endosc 2011;25:2865-70.

11. Harth KC, Rosen MJ. Endoscopic versus open component separation in complex abdominal wall reconstruction. Am J Surg 2010;199:342-6; discussion 6-7.

12. Lipman J, Medalie D, Rosen MJ. Staged repair of massive incisional hernias with loss of abdominal domain: a novel approach. Am J Surg 2008;195:84-8.

13. Lowe JBG, J. R.; Bowman, J. L.; Rohrich R. J.; Strodel W. E.;. Endoscopically assisted 'components separation' for closure of abdominal wall defects. Plast Reconstr Surg 2000:9.

14. Maas SM, de Vries Reilingh TS, van Goor H, de Jong D, Bleichrodt RP. Endoscopically assisted "components separation technique" for the repair of complicated ventral hernias. J Am Coll Surg 2002;194:388-90.

15. Switzer NJ, Dykstra MA, Gill RS, et al. Endoscopic versus open component separation: systematic review and meta-analysis. Surg Endosc 2014.

16. Slater NJ, van Goor H, Bleichrodt RP. Large and complex ventral hernia repair using "components separation technique" without mesh results in a high recurrence rate. Am J Surg 2014

17. Slater NJ, Montgomery A, Berrevoet F, et al. Criteria for definition of a complex abdominal wall hernia. Hernia 2014;18:7-17.

18. Garner JS. CDC guideline for prevention of surgical wound infections, 1985. Supersedes guideline for prevention of surgical wound infections published in 1982. (Originally published in November 1985). Revised. Infect Control 1986;7:193-200.

19. Rosen MJ, Williams C, Jin J, et al. Laparoscopic versus open-component separation: a comparative analysis in a porcine model. Am J Surg 2007;194:385-9.

20. Jensen KK, Henriksen NA, Jorgensen LN. Endoscopic component separation for ventral hernia causes fewer wound complications compared to open components separation: a systematic review and metaanalysis. Surg Endosc 2014.

21. Rosen MJ, Jin J, McGee MF, Williams C, Marks J, Ponsky JL. Laparoscopic component separation in the single-stage treatment of infected abdominal wall prosthetic removal. Hernia 2007;11:435-40. Epub 2007 Jul 24. 


\section{Chapter}

\section{A modified Chevrel technique for ventral hernia repair: long term results of a single center cohort.}

Elwin H.H. Mommers ${ }^{1,2}$, Bart J.M. Leenders ${ }^{3}$, Wouter K.G. Leclercq ${ }^{3}$, Tammo S. de Vries Reilingh ${ }^{1}$, Jan A. Charbon ${ }^{3}$

${ }^{1}$ Department of Surgery, Elkerliek Hospital, Helmond, The Netherlands ${ }^{2}$ Department of Surgery, Maastricht University Medical Center, Maastricht, The Netherlands

${ }^{3}$ Department of Surgery, Máxima Medical Center, Eindhoven, The Netherlands

Hernia 2017 March doi:10.1007/s10029-017-1602-2. Epub 2017 April $13^{\text {th }}$ ahead of print 


\section{ABSTRACT}

Background: To evaluate the short and long-term results after a modified Chevrel technique for midline incisional hernia repair, regarding surgical technique, hospital stay, wound complications, recurrence rate, and postoperative quality of life. These results will be compared to literature derived reference values regarding the original, and modified Chevrel techniques.

Methods: In this large retrospective, single surgeon, single center cohort all modified Chevrel hernia repairs between 2000 and 2012 were identified. Results were obtained by reviewing patients' medical charts. Postoperative quality of life was measured using the Carolina Comfort Scale. A multi-database literature search was conducted to compare the results of our series to literature-based reference values.

Results: One hundred and fifty-five patients (84 male, 71 female) were included. Eighty patients (52\%) had a large incisional hernia (width $\geq 10 \mathrm{~cm}$ ) according the definition of the European Hernia Society. Fourteen patients (9\%) underwent a concomitant procedure. Median length-of-stay was five days. Within 30 days postoperative 36 patients (23.2\%) had 39 postoperative complications of which 30 were mild (CDC I-II), and nine severe (CDC III-IV). Thirty-one surgical site occurrences were observed in thirty patients (19.4\%), of which the majority were seromas (16 patients $10.3 \%)$. There was no hernia related mortality during follow-up. Recurrence rate was $1.8 \%$ after a median follow-up of 52 months (12-128 months). Postoperative quality of life was rated excellent.

Conclusions: The modified Chevrel technique for midline ventral hernias results in a moderate complication rate, low recurrence rate and high rated postoperative quality of life. 


\section{INTRODUCTION}

Since primary closure of ventral abdominal wall hernias is accompanied with high recurrence rates, mesh-augmentation is an accepted evidence-based technique to ensure a strong and reliable abdominal wall herniorrhaphy. However, the optimal positioning of the mesh is controversial and heavily debated. ${ }^{1-3}$

Intra-abdominal mesh placement can potentially cause bowel adhesion and enterocutaneous fistulas due to the proximity of the mesh to the abdominal viscera. Sublay placement reduces the risk of these fistulas and adhesion formation, though the posterior rectus fascia must be closed in the midline to correctly place the mesh and prevent contact with the abdominal content. Additionally, it is not always possible to close the anterior rectus fascia in case of large hernias. Therefore, in large hernias sublay placement is frequently used in combination with other repairs such as the (endoscopic) anterior or posterior component separation technique. ${ }^{4,5}$ These techniques require dissection beyond the lateral border of the rectus muscles, increasing the risk of damage to the perforating epigastric arteries and nerves, seroma formation, skin necrosis, and bulging of the lateral abdominal wall. ${ }^{4,6}$ Despite the above-mentioned disadvantages, both intra-abdominal and sublay positions are preferred over onlay position of the mesh. The onlay position became disfavoured after large registry-based studies reported an alleged increased risk of seroma formation and wound infection when compared to other mesh positions, supposedly caused by extensive subcutaneous dissection. ${ }^{1,6}$ One of the onlay techniques included in these studies is the original Chevrel technique. This technique consists of an anterior fascia 'turnover' closed in the midline with an overlapping midline plasty, combined with a large onlay mesh augmentation sutured with overlap to the lateral part of the anterior rectus fascia, or even to the fascia of the oblique external muscle. ${ }^{4,7}$ To facilitate mesh placement, subcutaneous dissection beyond the lateral border of the anterior rectus fascia is often necessary with the original Chevrel technique.

In this report, we present a modified version of the Chevrel technique, according to an early technique description by Chevrel in $1979 .{ }^{8}$ Because of recurrences Chevrel turned this technique in to the well-known 'original' Chevrel technique published in 1986. ${ }^{7}$ Our modified Chevrel technique does not require such large subcutaneous dissection since the mesh is sutured to the remnant of the anterior rectus fascia with only one-and-a-half centimeters overlap. ${ }^{8}$ This results in a dual-layer repair where both the sutured anterior fascia turnover, and the onlay mesh provide support to the ventral abdominal wall. Therefore, the modified Chevrel technique offers the advantages of onlay mesh placement, potentially without the alleged disadvantage of increased wound complications caused by extensive subcutaneous dissection.

The aim of this study is to describe a modified Chevrel technique and to evaluate its complications, recurrences, and quality of life. These results will be compared to literature derived reference values. 


\section{METHODS}

A retrospective analysis of all patients receiving a midline incisional hernia repair using the modified Chevrel technique between 2000 and 2012 was performed. Patients were identified by searching ventral hernia repair codes in the electronic operation-theatre (OR) database. Patients undergoing concomitant procedures other than a component separation technique were also included. All patients were operated by a single surgeon (JC) in a single institution, the Máxima Medical Center, a large teaching hospital in Eindhoven, The Netherlands.

Noted endpoints were: patient characteristics, per- and postoperative details, postoperative complications according to the Modified Ventral Hernia Working Group Classification (VHWG), Clavien Dindo Classification, recurrences, and postoperative quality of life according to the Carolina Comfort Scale. ${ }^{9-12}$ Wound complications were reported as Surgical Site Occurrences (SSO), defined as any wound complication (haematoma, superficial and deep wound infection, abscess, seroma, fistula, and wound dehiscence). Infectious wound complications were reported separately as Surgical Site Infections (SSI), defined as abscess, infected seroma, superficial or deep wound infection.

Standard care consisted of at least one pre-operative visit at the outpatient clinic. During this visit, hernia size and treatment options were determined by physical examination. Pre-operative abdominal CT-scans were not performed routinely. Any patient with a midline ventral incisional hernia and sufficient healthy cutis and subcutis to cover the mesh and to close the skin was included. An existing enterostomy or the presence of an enterocutaneous fistula was no contra-indication.

All patients were invited to the outpatient clinic within four weeks after discharge. Follow-up was obtained by reviewing patient's medical records. Clinical examination by the surgeon (only if the medical record specifically described the condition of the abdominal wall), abdominal CT-scans and abdominal wall ultrasounds were all accepted as diagnostic instrument for recurrence. Abdominal CT-scans were reviewed by two authors simultaneous (JC, EM) to evaluate recurrence. In case of disagreement, a third author (TdVR) was consulted for arbitration. Any hernia at the site of the modified Chevrel repair was registered as a recurrence.

\section{Quality of life}

QoL measurements were performed with a validated Dutch version of the Carolina Comfort Scale (CCS), a hernia specific QoL questionnaire consisting of 23 items. ${ }^{9,11}$ The focus of the CCS is mesh related pain, mesh feeling, and movement limitation. The maximum score is 115 points and reflects the worst possible QoL, the minimum score is 0 and equals perfect QoL. In addition to the 'overall' QoL, the CCS reports three subdomains, 'mesh-sensation', 'pain', and 'movement impairment'. The maximum score per subdomain is 40 points for 'mesh sensation' and 'pain', and 35 points for 'movement 
impairment'. All living patients without a reoperation for a registered recurrence were contacted by letter during July 2015 to participate in the postoperative QoL evaluation. At that time, all patients were at least three years postoperative.

\section{Operative technique of a modified Chevrel repair}

Antibiotic prophylaxis (Cefuroxime $\left(\mathrm{Kefzo}^{\circledR}\right)$ 1500mg) is given intravenously. Following excision of the midline scar tissue, the hernia sac is opened and adhesiolysis is performed to facilitate movement and stretching of the abdominal wall along the abdominal content. The skin and subcutaneous tissue above the anterior rectus abdominis fascia are dissected until 2-3 cm medial of the lateral border of the rectus muscles, exposing the anterior rectus fascia. Depending on the width of the hernia, an elliptical incision is made in the anterior rectus fascia mostly between two and five centimeters from the medial edge of the rectus muscle. The medial part of the anterior rectus fascia is separated from the muscle fibers and tendinous intersections, and 'turned over' towards the midline. This procedure is repeated at the contralateral side. Any residue of the hernia sac is left alone or excised. Both the left and right 'turned over' anterior fascia can now be sutured in the midline with a continuous 2/0 polydioxanone (PDS) suture using small bites. ${ }^{13}$ This suture 'closes' the abdomen and provides the first 'layer' of the repair (figure 1). Any small defect in this new posterior layer is closed with an absorbable suture. The second layer is formed by a polypropylene mesh (Prolene ${ }^{\circledR}$, Ethicon Inc.; see table 1 for mesh details) sutured to the edge of the lateral part of the anterior fascia using two long double needled Prolene ${ }^{\circledR} 2 / 0$ sutures. One tied suture fixes the mesh at the cranial and one at the caudal meeting point of the left and right lateral part of the anterior rectus fascia. With small bites the mesh is sutured to the edge of the anterior fascia running from cranial and caudal, thereafter, meeting halfway where the cranial and caudal suture are tied together (figure 2). During this procedure, some tension is given on the suture line so the lateral abdominal wall muscles are stretched slightly. By manipulation of the tension and size of the mesh it is possible to influence the contour of the abdomen. The mesh must lay flat on the rectus muscle without any folds. Then the excess mesh is trimmed with scissors to leave a rim of approximately $1.5 \mathrm{~cm}$ lateral to the continuous suture. This rim is then sutured to the ventral side of the anterior rectus fascia using single Prolene ${ }^{\circledR} 2 / 0$ sutures. Some tailoring is necessary to provide a flat $1.5 \mathrm{~cm}$ overlap where connective tissue can grow into the mesh (figure 2). Two lowvacuum drains are placed in the subcutaneous space. The subcutaneous tissue and skin are closed with resorbable continuous sutures. The drains are removed when the production is less than $50 \mathrm{ml} / 24$ hours, mostly within three days. Patients with epidural analgesia are mobilised after removal of the catheter, approximately two to four days postoperative. All other patients are stimulated to walk the first postoperative day. Patients are advised to wear a comfortable abdominal binder for four weeks to prevent 
seroma formation. Normal daily activities are permitted, though heavy lifting was discouraged for six weeks.
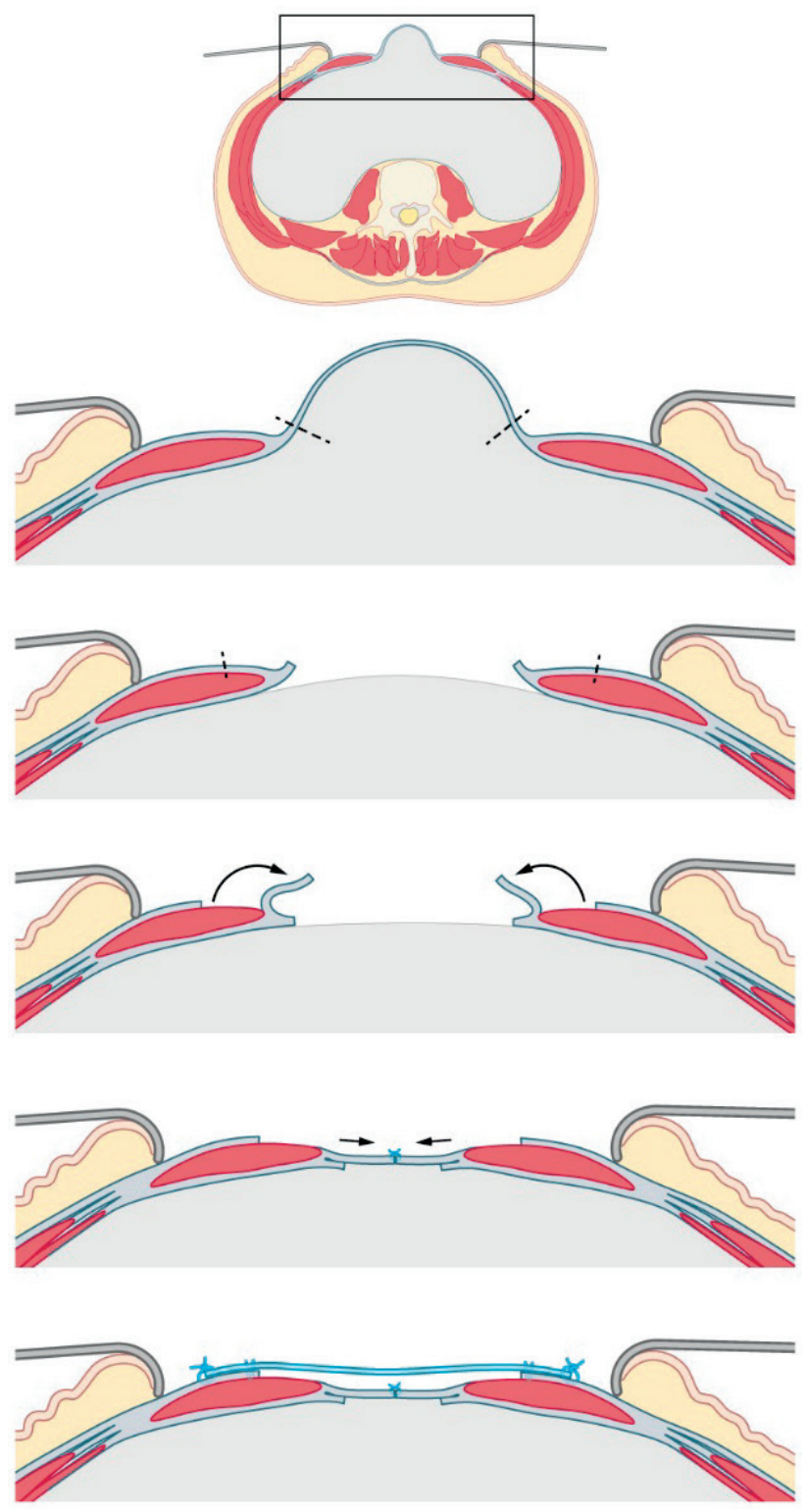

Figure 1 Schematic approach of a modified Chevrel technique for midline ventral hernia repair.

From top to bottom the following steps are depicted: anatomical situation with the hernia sac in situ; incision of the anterior rectus fascia and 'turn over'; suture of both turned over anterior fascia's in the midline to form the new posterior layer; fixation of the mesh with continues and single sutures to the lateral remnant of the anterior rectus fascia with $1,5 \mathrm{~cm}$ overlap. 


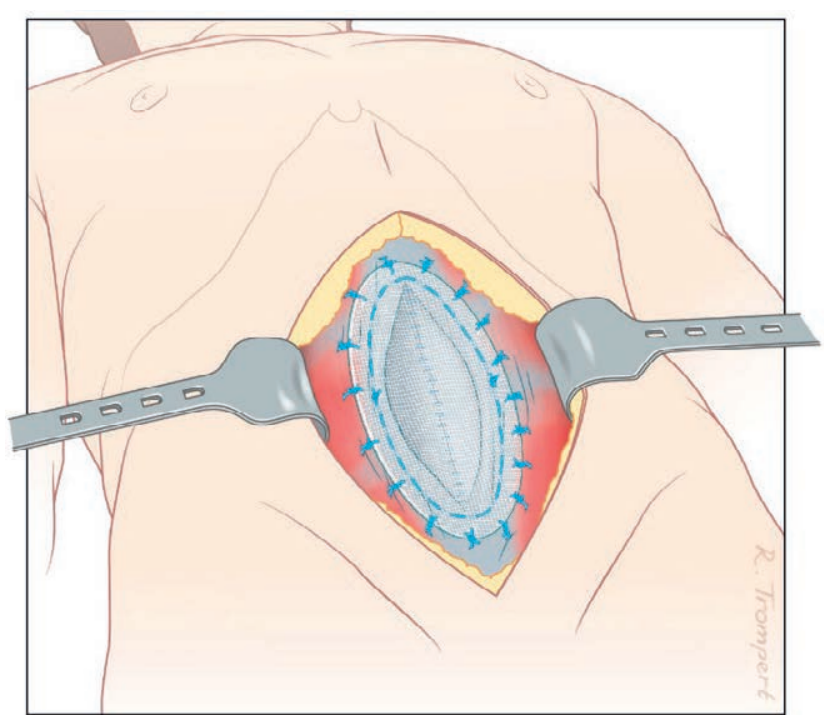

Figure 2 Schematic view of the anterior abdominal wall after mesh placement.

Ventral 'birds-eye-view' after the mesh is fixated to the lateral remnant of the anterior rectus fascia. There is a $1.5 \mathrm{~cm}$ overlap between the mesh and the anterior rectus fascia. A continuous suture is used to fixate the mesh to the lateral remnant of the anterior rectus fascia. Single sutures are used to assure that the overlap remains flat on the anterior rectus fascia to facilitate tissue ingrowths in the mesh. The new posterior layer can be seen through the mesh.

\section{Literature search}

To correctly interpret the results of this study and make any comparison to the existing body of evidence concerning the original and modified Chevrel techniques, a literature study was performed. The following databases (PubMed, Cochrane database, google scholar, Medline, EBSCO) were searched using the following terms: 'anterior fascia turnover' OR 'Chevrel method' OR 'Chevrel technique' OR 'anterior' OR 'frontal' OR 'fascia flip' OR 'shoelace technique' AND 'postoperative complications [MESH]' OR 'recurrence' OR 'hernia repair' OR 'hospital stay'.

All studies reporting postoperative complications, recurrence rate, or hospital stay after ventral hernia repair using either the original or a modified Chevrel techniques with anterior fascia turn-over were included. Only studies in English, French, Dutch, or German that accurately described the operation technique or referenced an article that described the operation technique and the exact limits of the subcutaneous dissection were included. No limitations to the year of publication were applied. 


\section{Statistical analysis}

All data was analysed using SPSS statistics for Windows, IBM corp. Armonk, NY, released 2013. Baseline characteristics, response rates, and recurrence rates were analysed using descriptive statistics. Statistical significance (a) was set at 0.05. Group comparisons were performed using independent samples Student's t test or Mann-Whitney U tests depending on the (non)parametric distribution of the data (Levene's test for equality). Correlations between VHWG and SSOs were calculated using bivariate correlations and expressed as Pearsons ' $r$ '.

\section{RESULTS}

Overall 297 patients underwent midline incisional hernia repair between 2000 and 2012. One hundred and fifty-five of these patients were operated using the modified Chevrel technique (see table 1 for demographics and type of mesh implants). The remaining patients, mostly with smaller hernias, were treated laparoscopically or with an open sublay repair. Fifty-two percent $(n=80)$ of all modified Chevrel repairs were done for the reconstruction of large ventral hernias (width $\geq 10 \mathrm{~cm}$ ) according to the definition of European Hernia Society (table 1). ${ }^{14}$ Fifty-seven patients received epidural analgesia during and after the surgery. Thirty-six patients had one prior hernia repair, and eleven patients had two or more previous repairs at the same site (in total 30\% repair for recurrences). All prior hernia repairs were performed using other techniques than the modified or original Chevrel repair.

Of the 155 patients, fourteen (9\%) received a concomitant procedure, and 110 patients (71\%) had a follow-up of at least 12 months (table 2). The median hospital stay was five days (range 2-95 days). 
*Wound infection after previous surgery, " Use of corticosteroids, chemoagents; ${ }^{\wedge}$ Previous repair of an abdominal aortic aneurism; ${ }^{\circ}$ Exact width measures missing in 17 of 155 patients. therapeutics, or immunosuppressive

Table 1 Demographic characteristics $(n=155)$

\begin{tabular}{|c|c|}
\hline Gender (male/female) & $84 / 71$ \\
\hline Smoking $(n)$ & 26 \\
\hline $\mathrm{BMI} \mathrm{kg} / \mathrm{m}^{2}$ (median, range) & $28\left(18-53 \mathrm{~kg} / \mathrm{m}^{2}\right)$ \\
\hline $\operatorname{COPD}(n)$ & 17 \\
\hline Diabetes Type 2 (n) & 20 \\
\hline Previous wound infection $(n)^{*}$ & 26 \\
\hline Wound healing impairing medication $(n)^{\#}$ & 3 \\
\hline Abdominal aortic aneurism(n)^ & 30 \\
\hline Defect width (median cm, range) & $10 \mathrm{~cm}(2-25 \mathrm{~cm})$ \\
\hline $\mathrm{W} 1<4 \mathrm{~cm}(\mathrm{n})^{\circ}$ & 7 \\
\hline$W 2 \leq 4-10 \mathrm{~cm}(\mathrm{n})^{\circ}$ & 51 \\
\hline$W 3 \geq 10 \mathrm{~cm}(\mathrm{n})^{\circ}$ & 80 \\
\hline Defect length (median cm, range) & $15 \mathrm{~cm}(3-35 \mathrm{~cm})$ \\
\hline Defect surface (median $\mathrm{cm}^{2}$, range) & $118 \mathrm{~cm}^{2}\left(5-550 \mathrm{~cm}^{2}\right)$ \\
\hline \multicolumn{2}{|l|}{ Type of mesh placed } \\
\hline Standard Polypropylenes (n) & 102 \\
\hline Small pore polypropylene (Marlex) (n) & 35 \\
\hline Large pore polypropylene (Vypro I) (n) & 18 \\
\hline
\end{tabular}

Table 2 Concomitant procedures $(n=)$ fifteen concomitant procedures with a modified Chevrel repair in the same session. *Sugarbaker technique $(n=1$, colostomy), Suturing hernia defect ( $n=1$, urostomy).

Cholec

3

Gastric banding 1

Parastomal hernia repair* 2

Hemicolectomy 1

Enterostomy removal 2

Enterocutaneous fistula removal 1

Oophorectomy 1

Removal of ovarian cyst 1

Sterilization 1

Stoppa procedure for bilateral groin hernia 2

\section{Complications}

During the first thirty postoperative days, 30 mild (CDC grade I-II) complications and nine severe (CDC grade III-IV) complications occurred in 36 patients (23.2\%) that underwent a modified Chevrel repair (table 3). Thirty-one SSOs were observed in thirty patients (19.4\%), of which the majority were seromas (16 patients $10.3 \%$ ). Eight seromas required fine needle-aspiration, one was drained under local anaesthetic, five required reinterventions (including two reinterventions one year postoperative due to recurrent seroma formation), and two disappeared spontaneously. Three of the nine severe complications within 30 days were seromas requiring reintervention, two were 
abscesses, two were severe wound infections, one was a postoperative bleeding, and one was an anastomotic leakage in a patient with concomitant ileostomy takedown. The revision of the anastomotic leakage was complicated by a massive bleeding, though after re-intervention an uneventful recovery followed.

The VHWG classification identified three groups within the population (grade I Low risk, 41\% ( $n=63)$; grade II Co-Morbid, 54\% ( $n=84)$; grade III Contaminated, 5\% ( $n=8)$ ). Complications occurred more frequently in the grade II and III patients. SSOs occurred significantly more with an increasing VHWG grade ( $r=0.209 p=0.009 ; \times 2 p=0.017)$. Within the VHWG grading scale or the overall population there were no statistically significant differences in the incidence of complications in hernias over or under $10 \mathrm{~cm}$ in width $(p=0.192)$.

Table 3 Complications $\leq 30$ days postoperative

\begin{tabular}{|c|c|c|c|c|c|c|c|}
\hline $\begin{array}{l}\text { VHWG } \\
\text { grade }\end{array}$ & Patients & CDC I-II (\%) & CDC III-IV (\%) & $\begin{array}{l}\text { Surgical Site } \\
\text { Occurrence } \\
(\%)^{\#}\end{array}$ & $\begin{array}{l}\text { Surgical Site } \\
\text { Infection (\%) }\end{array}$ & Seroma (\%) & $\begin{array}{l}\text { lleus/ } \\
\text { Pneumoni } \\
\text { a/ UTI (\%) }\end{array}$ \\
\hline Total & Total cohort $(n=155)$ & $30(17.4 \%)$ & $9(5.8 \%)$ & $31(19.4 \%)$ & $9(5.8 \%)$ & $16(10.3 \%)$ & $8(5.2 \%)$ \\
\hline \multirow[t]{4}{*}{ Grade I } & All patients $(n=63)$ & $8(9.5 \%)$ & $2(3.2 \%)$ & $6(9.5 \%)$ & $1(1.6 \%)$ & $3(4.8 \%)$ & $4(6.3 \%)$ \\
\hline & Width <10 cm $(\mathrm{n}=26)$ & $1(3.8 \%)$ & 0 & $1(3.8 \%)$ & 0 & $1(3.8 \%)$ & 0 \\
\hline & Width $\geq 10 \mathrm{~cm}(\mathrm{n}=28)$ & $6(14.3 \%)$ & $1(3.6 \%)$ & $4(14.3 \%)$ & 0 & $2(7.1 \%)^{*}$ & $3(10.7 \%)$ \\
\hline & Width unknown $(n=9)^{\pi}$ & $1(11.1 \%)$ & $1(11.1 \%)$ & $1(11.1 \%)^{*}$ & $1(11.1 \%)^{*}$ & 0 & $1(11.1 \%)$ \\
\hline \multirow[t]{4}{*}{ Grade II } & All patients $(n=84)$ & $20(22.6 \%)$ & $6(7.1 \%)$ & $22(26.2 \%)$ & $7(8.3 \%)$ & $12(14.3 \%)$ & $4(4.8 \%)$ \\
\hline & Width <10 cm (n=28) & 5 (17.9\%) & $1(3.6 \%)$ & $6(21.4 \%)$ & $1(3.6 \%)$ & $5(17.9 \%)^{*}$ & 0 \\
\hline & Width $\geq 10 \mathrm{~cm}(\mathrm{n}=49)$ & $15(28.6 \%)$ & $3(6.1 \%)$ & $14(28.6 \%)^{*}$ & $4(8.2 \%)^{*}$ & $7(14.3 \%)^{*}$ & $4(8.2 \%)$ \\
\hline & Width unknown $(n=7)^{\pi}$ & 0 & $2(28.6 \%)$ & $2(28.6 \%)^{*}$ & $2(28.6 \%)^{*}$ & 0 & 0 \\
\hline \multirow[t]{3}{*}{ Grade III } & All patients $(n=8)$ & $2(25 \%)$ & $1(12.5 \%)$ & $3(37.5 \%)$ & $1(12.5 \%)$ & $1(12.5 \%)$ & 0 \\
\hline & Width <10 cm (n=5) & $2(40 \%)$ & 0 & $2(40 \%)$ & $1(20 \%)$ & $1(20.0 \%)$ & 0 \\
\hline & Width $\geq 10 \mathrm{~cm}(\mathrm{n}=3)$ & 0 & $1(33.3 \%)$ & $1(33.3 \%)^{*}$ & 0 & 0 & 0 \\
\hline
\end{tabular}

$\%=$ percentage of population affected with complication (NB: CDC I-II and CDC III-IV complications may be observed more than once in one patient, hence the percentage of population affected is lower than the times the complication is observed divided by the group size); *One or more patients within this category required surgical intervention; ${ }^{\pi}$ Exact defect measurements were not available in 16 patients; UTI=Urinary Tract Infection; $C D C=$ Clavien-Dindo Classification; VHWG=Modified Ventral Hernia Working Group septic risk scale for Surgical Site Occurrences; ${ }^{*}=$ statistically significant difference between VHWG grades; Surgical Site Occurrence=infection, wound dehiscence, seroma, or development of an enterocutaneous fistula; Surgical Site Infection=deep or superficial wound infection, abscess, infected seroma.

\section{Long term complications}

Ten patients $(6 \%)$ reported persisting localized pain near the rim of the mesh, approximately one month postoperative. All these patients received one or more local anaesthetic injections (Lidocaine 1-2\%) that relieved their pain sufficiently. 
One patient with a severe wound infection was, after drainage, treated with vacuum assisted therapy, though developed a mesh infection more than 30 days postoperative, and later a recurrent hernia. Another patient, with correction of an enterocutaneous fistula as a concomitant procedure, developed a new enterocutaneous fistula after ten months, perhaps due to erosion or infection of a small part of the mesh. The wound healed after resection of the infected part of the mesh, without finding a bowel defect. Two patients with recurrent seroma formation needed surgical intervention after one and two years respectively. Yet another patient developed a suture fistula 16 months postoperative. After excision of a Prolene ${ }^{\circledR}$ suture, the wound healed.

\section{Recurrence}

The following methods were used for assessment of recurrence: clinical examination by the surgeon as retrieved from the patient's medical record which specifically described the condition of the abdominal wall (77\%), abdominal CT-scans (13\%), and abdominal wall ultrasounds (10\%). The overall median follow-up for the total cohort was 34 months (range 0-128 months, interquartile rage 8-62 months). Forty-five of the 155 patients had a follow-up shorter than twelve months and were not included in the longterm recurrences analysis. In this group of 45 patients no recurrences occurred. The remaining 110 patients had a median follow-up of 52 months (range 12-128 months, interquartile range 31-72 months), two of these patients developed a recurrence (1.8\%) after 13 and 15 months postoperative. Both patients had a large hernia (width $\geq 10 \mathrm{~cm}$ ). The first patient received a Vypro ${ }^{\circledR}$ mesh (large pore polypropylene with polyglactin, Ethicon Inc.) which failed after 13 months. During the re-intervention, it became apparent that the mesh was torn in half. The second patient had a postoperative wound infection that was initially treated with vacuum assisted therapy, though later progressed into a mesh infection that was treated by removing the infected part of the mesh. Initially, this resolved the problem of the infection, though over time the patient developed a recurrent hernia.

\section{Quality of life}

At the time of measurement 23 of the 155 patients were deceased, and two patients were operated for a recurrence using a different surgical technique. The remaining 130 patients were sent the CCS questionnaire. Ninety-six patients responded (response rate $74 \%)$. The median follow-up of these patients was 35 months. The average CCS score was seven on a scale of 115 (SD \pm 15$), 58$ patients (60\%) reported an excellent QoL-score of zero. A sub analysis of patients that reported any impairment in QoL (total score $\geq 1$; $\mathrm{n}=38)$ ) showed the following results: median overall QoL 12 (range 1-76), median sensation score 5 (range 0-27), median pain score 2 (range 0-26), and median movement score 4 (range 0-24). 


\section{Literature synopsis}

Twenty-two studies were identified through the database search, and four articles were identified through cross-reference (last search performed November 2016). A total of 26 articles were screened for eligibility. Fifteen studies were excluded, one due to language restrictions, and 14 because they did not meet the inclusion criteria. Eventually, ten studies (eleven including the current series) were included (figure 3 and table 4). ${ }^{7,8,15-22}$ Studies were divided in two groups, the original Chevrel technique and the modified Chevrel techniques, depending on the overlap of the mesh and the amount of subcutaneous dissection. The modified Chevrel repairs used a trimmed mesh and no subcutaneous dissection lateral of the anterior rectus sheath.

The current evidence as displayed in table 4 consists of multiple small case series with heterogeneous populations in term of hernia size, location, origin, complexity, and follow-up method. The median and average follow-up varies between the studies, though is mostly longer than one year.

Wound complication rates varied from $0-25 \%$ in de modified Chevrel group, and from $9-44 \%$ in de original Chevrel group. Hospital stay was slightly shorter in the modified Chevrel group (5-6 vs 5-11 days). The recurrence rates in the modified Chevrel group were $0-2 \%$ compared to $0-33 \%$ in the original group, though the defect size in two of the five studies describing the modified Chevrel technique were not measured objectively.

Based on the limited evidence available, the results of the current series are comparable with previously published studies of the modified Chevrel techniques. Previous studies have never reported any recurrences using the modified Chevrel technique. This could be due to the relative short follow-up, retrospective methodology, small populations, or due to publication bias, though, considering the low recurrence rate in the current series, recurrences are rare after a modified Chevrel technique. 


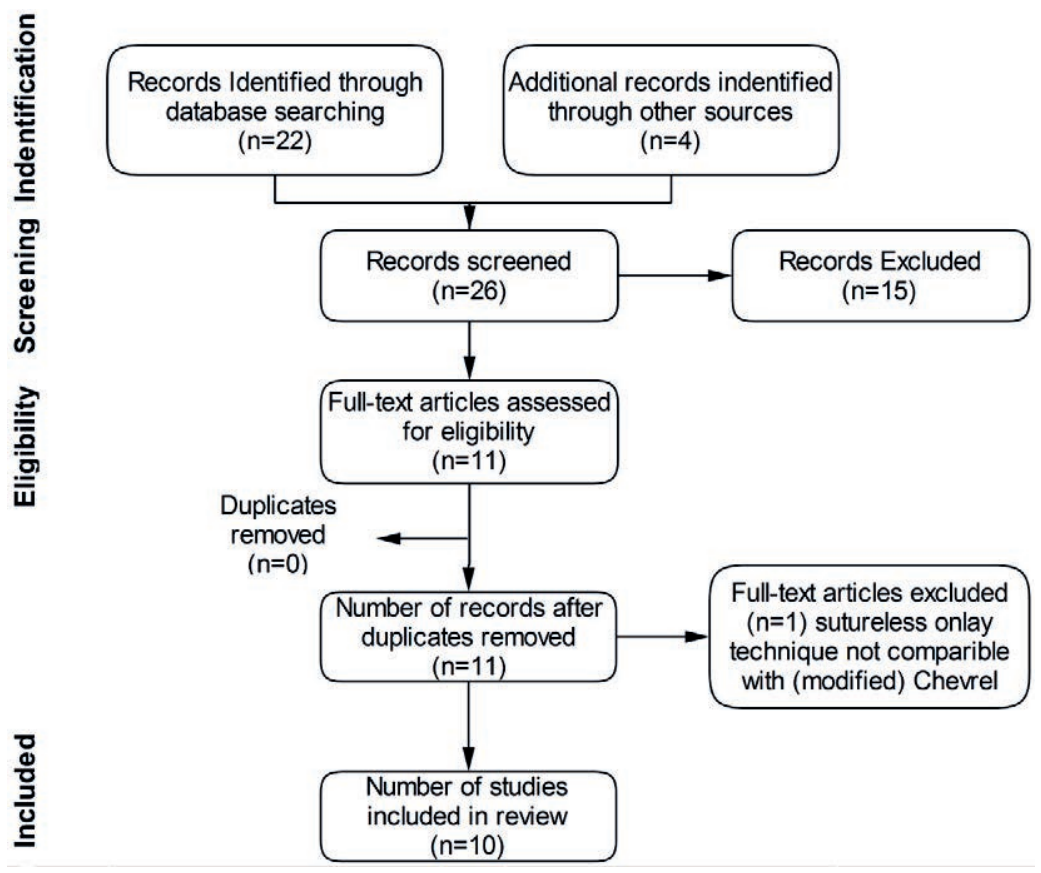

Figure 3 Flow of studies through literature analysis

( $n=$ total number of studies; This flowchart is in accordance with the 2009 PRISMA statement.

Table 4 Current literature describing the original and modified Chevrel technique

\begin{tabular}{|c|c|c|c|c|c|c|}
\hline \multicolumn{2}{|l|}{ Population } & $\begin{array}{l}\text { Defect } \\
\text { size }\end{array}$ & Hospital stay & $\begin{array}{l}\text { Wound } \\
\text { complications }\end{array}$ & Follow-Up & Recurrences \\
\hline \multicolumn{7}{|l|}{ Original Chevrel technique } \\
\hline J.P. Chevrel $(1986)^{7}$ & 50 & $1-20 \mathrm{~cm}$ & NR & $18(36 \%)$ & $\begin{array}{l}10 \text { months }-114 \\
\text { months }\end{array}$ & $4(8 \%)$ \\
\hline J.R. San Pio $(2003)^{21}$ & 67 & $N R^{*}$ & NR & $6(9 \%)$ & 5.7 years (range $0-17$ ) & $10(15 \%)$ \\
\hline S. Licheri $(2008)^{18}$ & 64 & $5-15 \mathrm{~cm}$ & 10 days $(2-21)$ & $17(26.5 \%)$ & 54 months & $2(3.5 \%)$ \\
\hline A. Forte $(2011)^{16}$ & 9 & $5-10 \mathrm{~cm}$ & 9 days (5-18) & $4(44 \%)$ & NR & $3(33 \%)$ \\
\hline F. Marchesi $(2011)^{15}$ & 21 & $\pm 8 \mathrm{~cm}$ & 11 days (4-58) & $7(33 \%)$ & 17 months & $0(0 \%)$ \\
\hline E.I. Hodgman $(2016)^{22}$ & 123 & $65 \mathrm{~cm}^{2}$ & 5 days $(0-31)$ & $40(34 \%)$ & 18 months & $6(5 \%)$ \\
\hline \multicolumn{7}{|c|}{ Modified Chevrel technique's } \\
\hline J.P. Chevrel $(1979)^{8}$ & 12 & $>10 \mathrm{~cm}$ & NR & $3(25 \%)$ & 30 months -8 years & $0(0 \%)$ \\
\hline M.S. Whiteley $(1998)^{19}$ & 10 & $N R^{*}$ & NR & $0(0 \%)$ & 17 months & $0(0 \%)$ \\
\hline H.S. Khaira $(2001)^{20}$ & 35 & $N R^{*}$ & 6 days $(1-27)$ & $8(22 \%)$ & $\begin{array}{l}20 \text { months, (range 6- } \\
54 \text { ) }\end{array}$ & $0(0 \%)$ \\
\hline M.A. Joshi $(2015)^{17}$ & 30 & $>4 \mathrm{~cm}$ & 5 days $\mathrm{SD} \pm 4$ & $3(10 \%)$ & 12 months & $0(0 \%)$ \\
\hline E.H.H. Mommers (2016) & 155 & $10 \mathrm{~cm}$ & 5 days (2-95) & $27(19 \%)$ & 52 months (median) & $2(2 \%)^{*}$ \\
\hline
\end{tabular}

Characteristics of all included studies; Wound complications = wound/mesh infection, seroma, haematoma, or wound dehiscence; NR = not reported in the publication; *Only patients with a follow-up $\geq 12$ months were included ( $n=110)$, values can be average, median or range, depending on the original publication. 


\section{DISCUSSION}

Previous studies have reported a relatively high wound complication rate after the original Chevrel technique, which was contributed to the extensive subcutaneous dissection to facilitate overlap of the mesh beyond the lateral border of the rectus abdominis muscles. This was also demonstrated in the meta-analysis of Timmermans et al. from 2014, though this meta-analysis does not distinguish between different types of onlay repair. ${ }^{1}$ The modified Chevrel technique requires less subcutaneous dissection and a smaller mesh. This could account for the reduced wound complication rate in comparison with the original technique as observed in this study. The modified Chevrel technique can be used for any patient with a sufficient amount of healthy skin and subcutaneous tissue to cover the mesh and can even be used in patients with an enterostomy, urostomy, or enterocutaneous fistula.

The most frequent postoperative complication in this series was seroma formation. Seromas form in nearly $100 \%$ of the mesh-based repairs, and are easily detected after onlay mesh placement due to the proximity of the dissection plane and mesh to the skin. $^{23,24}$ Therefore, the authors would argue that seroma 'detection', rather than seroma formation, is increased in onlay mesh placement. With sublay or intra-abdominal mesh placement the mesh and dissection plane are situated on a deeper level, meaning seromas are often not clinically detected, although radiologically, they are present. Therefore, the high number of seromas usually reported after onlay placement should not be an argument to discard the technique, especially not since the wound complication rate as observed in our series is comparable with sublay mesh placement, and the recurrence rate is low. ${ }^{6}$

Previous studies have demonstrated that onlay mesh placement could lead to less recurrences when compared to sublay placement, though the evidence is not conclusive. A prospective five-year trial of Wéber et al. showed recurrence rates after onlay mesh placement to be half of the recurrence rates after sublay mesh placement (12\% onlay vs $22 \%$ sublay, $\mathrm{p}<0.05) .{ }^{25}$ A 2008 Cochrane meta-analysis pre-dates the abovementioned trial, though did show a clear trend towards lower recurrence rates in onlay mesh placement compared to sublay placement (RR0.66, 95\% $\mathrm{Cl}[0.35-1.25]) .{ }^{26}$ On the other hand, Timmermans et al. showed a trend towards lower recurrence rates in sublay repair (OR 2.41 95\% Cl [0.99-5.88]) in their 2014 meta-analysis. ${ }^{1}$ In the present series, we observed only two recurrences (1.8\%). The authors emphasize the importance of a meticulous continuous suturing technique of the mesh to the lateral remnant of the anterior rectus fascia to replicate these results. The meticulous suturing technique is the most time-consuming part of the procedure that will take approximately two hours in total.

Our study shows excellent postoperative quality of life after the modified Chevrel repair. An overall average score of 7/115 indicates only minimal impairment of QoL. 
Those patients that did experience an impairment of their daily function mainly reported that they 'felt the presence of the mesh' during daily activity.

In our series, we observed ten patients who had localized (1-2 fingerbreadths) abdominal wall pain somewhere along the suture line, at the rim of the mesh over the anterior rectus fascia. The clinical presentation was comparable to the anterior cutaneous nerve entrapment syndrome (ACNES). ${ }^{27}$ Similar to the proposed treatment of ACNES patients, these hernia repair patients could be treated with local anaesthetic injections at the location of the maximum pain point. The phenomenon of localized abdominal wall pain might be contributed to traction on, or damage to the intercostal cutaneous nerve end branches in the suture line, or irritation by the mesh. ${ }^{27}$

The modified Chevrel technique offers a comprehensive approach to all types of midline ventral hernias, if there is sufficient healthy skin to cover the mesh. If this technique alone is not sufficient to close the defect, it can easily be combined with a minimally invasive or endoscopically assisted component separation technique. ${ }^{5}$ However, since the fascia turnover creates a new posterior layer that is wider than the 'anatomical' posterior rectus fascia, this technique on its own can bridge wider defects than the Rives-Stoppa approach. Therefore, a sublay position of the mesh will require additional techniques, such as component separation, sooner than the modified Chevrel repair. Additionally, due to the onlay placement of the mesh continuity of both the anterior rectus fascia and the new posterior layer are ensured, resulting in a double layer repair. In our series, we could conveniently close defects of $15 \mathrm{~cm}$ width using only the modified Chevrel technique. Between 2000 and 2012 there were three modified Chevrel repairs combined with additional component separation procedures, these patients were not included in this study.

Due to the retrospective nature of this study some limitations must be accentuated. Firstly, retrospective studies are more susceptible to report bias due to their dependence on historical descriptions. Secondly, no baseline measurement for QoL could be performed and the postoperative measurement was cross-sectional meaning that patients were at different post-operative intervals. The CCS questionnaire specifically focuses on mesh related complaints and leaves no room for 'other pain' or discomfort not caused by the mesh. Therefore, the incidence of 'mesh pain' or 'mesh sensation' might be overrepresented since not all abdominal wall pain experienced by the patient is caused by the mesh, nor does the CCS questionnaire distinguish different types of pain.

Thirdly, a meta-analysis of the literature review was deemed unfit by the authors due to the small patient samples, lack of proper RCT's, and heterogeneity in outcome variables. Since all patients were operated by the same surgeon, in the same institution, performance bias was practically excluded. Considering the aforementioned limitations, the authors feel that the following conclusion can be drawn from this study. 


\section{CONCLUSION}

The modified Chevrel technique for midline ventral hernia repair provides a durable repair with a $19.4 \%$ wound complication rate, of which the majority are seromas, and a low recurrence rate of $1.8 \%$ after a median follow-up of 52 months, and a high rated post-operative quality of life. Overall, these results exceed the result published on the original Chevrel technique. Indicating that this modified Chevrel technique leads to favourable results and should have a place in modern ventral hernia repair.

\section{ACKNOWLEDGEMENTS}

The authors would like to acknowledge and thank Drs. R. den Dekker for his support with managing and editing the study database and Dr. Rudy Roumen for his support in revising the manuscript.

\section{DISCLOSURE STATEMENT}

All other authors declare that they have no conflict of interest.

\section{ETHICAL APPROVAL}

All procedures performed in studies involving human participants were in accordance with the ethical standards of the institutional and/or national research committee, and with the 1964 Helsinki declaration and its later amendments or comparable ethical standards.

\section{STATEMENT OF HUMAN AND ANIMAL RIGHTS}

This article does not contain any studies with animals performed by any of the authors. 


\section{REFERENCES}

1. Timmermans L, de Goede B, van Dijk SM, Kleinrensink GJ, Jeekel J, Lange JF. Meta-analysis of sublay versus onlay mesh repair in incisional hernia surgery. Am J Surg 2014;207:980-8.

2. Luijendijk RW, Hop WC, van den Tol MP, et al. A comparison of suture repair with mesh repair for incisional hernia. N Engl J Med 2000;343:392-8.

3. Deerenberg EB, Timmermans L, Hogerzeil DP, et al. A systematic review of the surgical treatment of large incisional hernia. Hernia 2015;19:89-101. doi: 10.1007/s10029-014-1321-x. Epub 2014 Nov 8.

4. de Vries Reilingh TS, van Goor H, Rosman C, et al. "Components separation technique" for the repair of large abdominal wall hernias. J Am Coll Surg 2003;196:32-7.

5. Mommers EH, Wegdam JA, Nienhuijs SW, de Vries Reilingh TS. How to perform the endoscopically assisted components separation technique (ECST) for large ventral hernia repair. Hernia : the journal of hernias and abdominal wall surgery 2016;20:441-7.

6. de Vries Reilingh TS, van Geldere D, Langenhorst B, et al. Repair of large midline incisional hernias with polypropylene mesh: comparison of three operative techniques. Hernia : the journal of hernias and abdominal wall surgery 2004;8:56-9.

7. Chevrel JP, Morquette CDH. Traitment des éventrations abdominales médianes par autoplastie musculaire et prothèse pré-musculo-aponévrotique. Chirurgie (Mémoires de l'Académie) 1986;9:7.

8. Chevrel JP. Traitment des grandes éventrations médianes par plastie en paletot et prothèse. La Nouvelle Presse Médicale 1979;8:695-6.

9. Heniford BT, Walters AL, Lincourt AE, Novitsky YW, Hope WW, Kercher KW. Comparison of generic versus specific quality-of-life scales for mesh hernia repairs. J Am Coll Surg 2008;206:638-44. doi: 10.1016/j.jamcollsurg.2007.11.025. Epub 8 Feb 1.

10. Hope WW, Lincourt AE, Newcomb WL, Schmelzer TM, Kercher KW, Heniford BT. Comparing quality-oflife outcomes in symptomatic patients undergoing laparoscopic or open ventral hernia repair. J Laparoendosc Adv Surg Tech A 2008;18:567-71.

11. Nielsen K, Poelman MM, den Bakker FM, van der Ploeg T, Bonjer HJ, Schreurs WH. Comparison of the Dutch and English versions of the Carolinas Comfort Scale: a specific quality-of-life questionnaire for abdominal hernia repairs with mesh. Hernia 2014;18:459-64. doi: 10.1007/s10029-013-1173-9. Epub 2013 Oct 29.

12. Kanters AE, Krpata DM, Blatnik JA, Novitsky YM, Rosen MJ. Modified hernia grading scale to stratify surgical site occurrence after open ventral hernia repairs. J Am Coll Surg 2012;215:787-93.

13. van Ramshorst GH, Klop B, Hop WC, Israelsson LA, Lange JF. Closure of midline laparotomies by means of small stitches: practical aspects of a new technique. Surg Technol Int 2013;23:34-8.

14. Muysoms FE, Miserez M, Berrevoet F, et al. Classification of primary and incisional abdominal wall hernias. Hernia 2009;13:407-14. doi: 10.1007/s10029-009-0518-x. Epub 2009 Jun 3.

15. Marchesi F, Pinna F, Cecchini S, Sarli L, Roncoroni L. Prospective comparison of laparoscopic incisional ventral hernia repair and Chevrel technique. Surg Laparosc Endosc Percutan Tech 2011;21:306-10.

16. Forte A, Zullino A, Manfredelli S, Montalto G, Bezzi M. Incisional hernia surgery: report on 283 cases. Eur Rev Med Pharmacol Sci 2011;15:644-8.

17. Joshi MA, Singh MB, Gadhire MA. Study of the outcome of modified shoelace repair for midline incisional hernia. Hernia : the journal of hernias and abdominal wall surgery 2015;19:503-8.

18. Licheri S, Erdas E, Pisano G, Garau A, Ghinami E, Pomata M. Chevrel technique for midline incisional hernia: still an effective procedure. Hernia : the journal of hernias and abdominal wall surgery 2008;12:121-6.

19. Whiteley MS, Ray-Chaudhuri SB, Galland RB. Combined fascia and mesh closure of large incisional hernias. J R Coll Surg Edinb 1998;43:29-30.

20. Khaira HS, Lall P, Hunter B, Brown JH. Repair of incisional hernias. J R Coll Surg Edinb 2001;46:39-43.

21. San Pio JR, Damsgaard TE, Momsen O, Villadsen I, Larsen J. Repair of giant incisional hernias with polypropylene mesh: a retrospective study. Scand J Plast Reconstr Surg Hand Surg 2003;37:102-6. 
22. Hodgman El, Watson MJ. Revisiting the Anterior Rectus Sheath Repair for Incisional Hernia: A 10-Year Experience. World Journal of Surgery 2016:1-9.

23. Susmallian S, Gewurtz G, Ezri T, Charuzi I. Seroma after laparoscopic repair of hernia with PTFE patch: is it really a complication? Hernia 2001;5:139-41.

24. Morales-Conde S. A new classification for seroma after laparoscopic ventral hernia repair. Hernia 2012;16:261-7.

25. Weber G, Baracs J, Horvath OP. ["Onlay" mesh provides significantly better results than "sublay" reconstruction. Prospective randomized multicenter study of abdominal wall reconstruction with sutures only, or with surgical mesh--results of a five-years follow-up]. Magy Seb 2010;63:302-11. doi: 10.1556/MaSeb.63.2010.5.3.

26. den Hartog D, Dur AH, Tuinebreijer WE, Kreis RW. Open surgical procedures for incisional hernias. Cochrane Database Syst Rev 2008:CD006438. doi: 10.1002/14651858.CD006438.pub2.

27. Boelens OB, Scheltinga MR, Houterman S, Roumen RM. Management of anterior cutaneous nerve entrapment syndrome in a cohort of 139 patients. Ann Surg 2011;254:1054-8. 


\section{Chapter}

\section{Evaluating the 'standard' polypropylene and polyester mesh in terms of shrinkage, tissue ingrowth, and collagen deposition.}

Elwin H.H. Mommers ${ }^{1 *}$, Kevin W.Y. van Barneveld ${ }^{1 *}$, Marion J. Gijbels ${ }^{2}$, Tammo S. de Vries Reilingh ${ }^{3}$, Marc H.F. Schreinemacher ${ }^{1}$, Nicole D. Bouvy ${ }^{1}$

${ }^{1}$ Department of General Surgery and NUTRIM school of Nutrition, Toxicology, and Metabolism, Maastricht University Medical Center, Maastricht, The Netherlands

${ }^{2}$ Department of Molecular Genetics, Cardiovascular Research Institute Maastricht, The Netherlands \& Department of Medical Biochemistry, Academic Medical Center, University of Amsterdam, The Netherlands

${ }^{3}$ Department of General Surgery, Elkerliek Hospital, Helmond, The Netherlands

Submitted to 'Hernia' 


\section{ABSTRACT}

Background: Many mesh characteristics influence clinical performance of surgical meshes. Given the vast number of variables and continuous development of meshes, comparing individual meshes is difficult. The aim of this study is to compare four 'standard' commercially available polypropylene and polyester meshes in a subcutaneous rat model to analyse differences in clinical performance.

Methods: Four commercially available meshes; Parietex (Covidien-Medtronic, Mansfield, USA), Prolene ${ }^{\circledR}$, Prolene Soft ${ }^{\circledR}$, and UItraPro (Ethicon, Johnson \& Johnson, Somerville, New Jersey, USA) were tested in a subcutaneous rat model. Eighty male Wistar Han outbred rats were included in this study. Noted endpoints were: mesh shrinkage, inflammatory response, tissue ingrowth, and neovascularization.

Results: After 90 days, the Parietex polyester mesh displayed no shrinkage compared to baseline. There was no difference in mesh ingrowth between the different types of meshes. The Prolene Soft mesh showed profound inflammation after 90 days compared to the remaining meshes. Despite the low number of inflammatory cells, the UltraPro mesh showed significantly higher collagen type 1:3 ratios indicating ongoing tissue remodelling. No significant differences in neovascularization were observed.

Conclusion: Parietex ${ }^{\oplus}$ polyester mesh shows hardly any mesh shrinkage when placed in a subcutaneous pocket and performs significantly better in this outcome than any of the polypropylene meshes included in this study. Tissue incorporation was similar for all the included meshes, regardless of mesh weight, pore size or mesh material. Parietex and Prolene showed high collagen type 1 deposition around the mesh, indicating mature collagen deposition without chronic tissue remodelling. 


\section{INTRODUCTION}

The use of mesh has become standard for ventral abdominal wall herniorrhaphy to ensure a durable repair of hernias over $3 \mathrm{~cm}$ wide. ${ }^{1}$ The first surgical meshes for hernia repair were strong, heavyweight meshes with a small pore size. ${ }^{2}$ These meshes led to a pronounced formation of fibrosis, resulting in a thick fibrotic plate. ${ }^{3,4}$ Over the years, the insight has grown that a thick and rigid fibrotic plate may cause adverse effects such as pain and foreign body sensation. Therefore, research has focused on developing meshes with low foreign body reaction, reduced chronic inflammation, and high collagen $1: 3$ ratios to create a thin layer of strong fibrotic tissue to prevent the aforementioned complications. ${ }^{5,6}$ Recent insights indicate that many variables can alter the foreign body response to a surgical mesh, such as the mesh material, mesh weight, number of filaments, presence of a surface modification, and pore size. ${ }^{7}$ Given the large number of variables, and continuous development of new meshes, surgeons struggle with comparing different meshes. Comparing individual meshes is becoming nearly impossible. In 2012 there were 166 different prosthetic devices for hernia repair available, although recent numbers are unavailable, current estimations range from 200 to 300 commercially available meshes. ${ }^{8}$ Many manufacturers offer different meshes with comparable characteristics, though previous studies have shown that meshes with seemingly comparable characteristics, such as mesh weight or pore size, can have different clinical performance in terms of mesh shrinkage, inflammatory reaction to the mesh, and tissue ingrowth. ${ }^{9}$

This study includes three commercially available 'standard' monofilament polypropylene meshes; Prolene ${ }^{\star}$, Prolene Soft ${ }^{\circledR}$, and UltraPro (all Johnson \& Johnson ') with different mesh weight and pore size and compared them to a multifilament polyester mesh, Parietex (Covidien-Medtronic ${ }^{\circledR}$ ). The aim of this study is to compare the aforementioned polypropylene meshes to a standard polyester mesh in terms of shrinkage, inflammatory response, mesh ingrowth, collagen formation, and neovascularization in a subcutaneous rat model after 28 and 90 days follow-up.

\section{METHODS}

Approval of the animal ethics committee of the Maastricht University Medical Center was obtained before commencement of this study. This study was performed in accordance with the Dutch Experiments on Animal Act (EAA).

\section{Meshes}

This prospective comparative animal study included four commercially available polyester and polypropylene meshes with varying weight and pore size; Parietex (Covidien- 
Medtronic, Mansfield, USA), Prolene ${ }^{\oplus}$, Prolene Soft ${ }^{\circledR}$, and UltraPro (Ethicon, Johnson \& Johnson, Somerville, New Jersey, USA) (table 1). Noted endpoints were; mesh ingrowth, tissue inflammation, neovascularization, and mesh shrinkage, measured after 28 and 90 days follow-up. Statistical power was calculated using the following variables, $\alpha=0.05$, $\beta=0.20$, clinically relevant difference $=20 \%$, expected variance $=15 \%$, and resulted in a group size of ten animals per group/timepoint. ${ }^{10}$ Including four different meshes and two follow-up periods in the study, total inclusion was set at eighty male Whistar Han IGS rats, weighing 250-350 gr. (Charles River Laboratories).

Table 1 Mesh characteristics

\begin{tabular}{|c|c|c|c|c|c|}
\hline Mesh & Basic material & Surface modification & Weight & Pore size & Manufacturer \\
\hline Prolene & $\begin{array}{l}\text { Monofilament } \\
\text { Polypropylene }\end{array}$ & None & $\begin{array}{l}\text { Heavyweight, } \\
80-85 \mathrm{~g} / \mathrm{m}^{2}\end{array}$ & $\begin{array}{l}1.6 \times 1.0 \\
\mathrm{~mm}^{11}\end{array}$ & $\begin{array}{l}\text { Ethicon (Johnson \& } \\
\text { Johnson) }\end{array}$ \\
\hline Prolene Soft ${ }^{\circledR}$ & $\begin{array}{l}\text { Monofilament } \\
\text { Polypropylene }\end{array}$ & None & $\begin{array}{l}\text { Lightweight, } \\
41 \mathrm{~g} / \mathrm{m}^{2}\end{array}$ & $\begin{array}{l}2.4 \times 1.7 \\
\mathrm{~mm}\end{array}$ & $\begin{array}{l}\text { Ethicon (Johnson \& } \\
\text { Johnson) }\end{array}$ \\
\hline Parietex & $\begin{array}{l}\text { Multifilament } \\
\text { Polyester }\end{array}$ & None & $\begin{array}{l}\text { Lightweight, } \\
46 \mathrm{~g} / \mathrm{m}^{2}\end{array}$ & $\begin{array}{l}2.4 \times 2.0 \\
\mathrm{~mm}\end{array}$ & Covidien-Medronic \\
\hline UltraPro & $\begin{array}{l}\text { Monofilament } \\
\text { Polypropylene }\end{array}$ & $\begin{array}{l}\text { Absorbable monofilament } \\
\text { poliglecaprone composite } \\
\text { (Monocryl') }\end{array}$ & $\begin{array}{l}\text { Lightweight, } \\
28 \mathrm{~g} / \mathrm{m}^{2}\end{array}$ & $\begin{array}{l}2.3 \times 3.4 \\
\mathrm{~mm}\end{array}$ & $\begin{array}{l}\text { Ethicon (Johnson \& } \\
\text { Johnson) }\end{array}$ \\
\hline
\end{tabular}

Characteristics of the included meshes; none = no surface modification was applied to the mesh; pore size was extracted from the product manual or from studies measuring pore size using scanning electron microscopy.

\section{Surgical procedure}

Animals were anesthetized via inhalation of Isoflurane (Forene, Abbott Laboratories, induction 3.5 vol.\%, maintenance 2.0-2.5 vol.\%) and received peri-operative analgesia in the form of Buprenorphine (Temgesic ${ }^{\circledR}$ Schering-Plough) $0.1 \mathrm{mg} / \mathrm{kg}$. The abdominal skin was shaved, disinfected with iodine $1 \%$, and covered with sterile drapes. A subcutaneous pocket of approximately $2.5 \times 3.5 \mathrm{~cm}$ was created via a $4 \mathrm{~cm}$ paramedian, craniocaudal incision over the abdomen. The subcutaneous pocket was created by blunt dissection in the subcutaneous plane between the skin, the anterior rectus fascia and external oblique muscle. The abdominal musculature was left completely intact. A sterile piece of mesh measuring $20 \times 30 \mathrm{~mm}$ was placed inside the subcutaneous pocket and fixated to the abdominal wall via six polypropylene 4-0 sutures (Prolene ${ }^{\circledR}$, Ethicon, Johnson \& Johnson) one on each corner, and one in the middle of the lateral borders of the mesh. The skin incision was closed with an intracutaneously running poliglecaprone 4-0 suture (Monocryl; ; Ethicon, Johnson \& Johnson).

\section{Sample collection}

At completion of follow-up, animals were euthanized via carbon dioxide overdose. The skin overlying the ventral abdominal wall musculature was incised through a U-shaped 
incision extending lateral and caudal to the mesh, opening the subcutaneous pocket in which the mesh was placed. The mesh in all animals was checked for signs of infection, defined as clinical infection with pus or serous pockets alongside the mesh with clinical signs of inflammation. Mesh size was measured in millimeters, and standardized photographs were taken for evaluation. Samples of the abdominal wall including the entire mesh were collected via block excision. The block was divided in half along the craniocaudal axis, cutting the mesh in two pieces of $10 \times 30 \mathrm{~mm}$. One half was used for histological examination, the other for measuring mesh ingrowth.

\section{Mesh ingrowth}

The strength of mesh ingrowth was defined as the maximum strength required to disrupt a $150 \mathrm{~mm}^{2}(10 \times 15 \mathrm{~mm})$ piece of mesh from the abdominal wall. One half of the block excision specimen was used for this measurement. Tissue was removed from the mesh until an exact overlap of the mesh with the abdominal wall of $150 \mathrm{~mm}^{2}(10 \times 15 \mathrm{~mm})$ remained. Any sutures in the specimen were removed. The specimen was placed in a traction tensiometer (Advanced Force Gauge ${ }^{\circledR}$ Mecmesin, UK), fixating the soft tissue of the specimen on one side, and the mesh on the other side (figure 1). The traction tensiometer applied an increasing

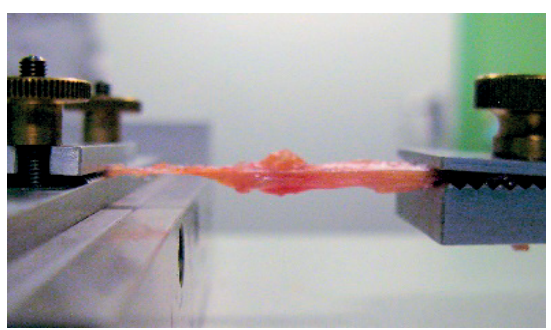

Figure 1 Tensile strength measurement

Tensile strength measurement showing the mesh without soft tissue in the clamp on the left and the abdominal wall without mesh in the clamp on the right. Both clamps are attached to a force gauge that records the maximum tensile strength whilst the clamps are pulled apart at a constant rate of $0.5 \mathrm{~mm} / \mathrm{s}$. tension on the specimen by pulling apart the mesh and the soft tissue at a steady rate of $0.5 \mathrm{~mm} / \mathrm{s}$. The maximum tensile strength required for complete separation of the abdominal wall and the mesh was recorded.

\section{Tissue inflammation}

Prior to microscopic evaluation all specimens were fixated in formaldehyde $4 \%$, dehydrated using increasing concentrations of ethanol, embedded in paraffin, and sliced in 5 $\mu \mathrm{m}$ sections. All samples were analysed by a single experienced pathologist (MG), blinded for sample allocation. A hematoxylin-eosin staining was performed and inflammation was measured via semi-quantitative scores: not present, slightly present (only directly around mesh filaments), moderately present (inflammation in between filaments), abundantly present (continuous thick layer of reactive tissue surrounding the mesh). In addition to the overall inflammatory response the presence of fibroblasts, giant cells, and macrophages were individually assessed using the same scale, previously used by Schreinemacher et al. ${ }^{12}$ 


\section{Collagen formation}

A picro Sirius red stain was used to visualized collagen fibers. Sections were exposed to a $0.1 \%$ solution of Sirius red in saturated aqueous picric acid for 90 minutes, followed by two minutes of washing in $0.01 \mathrm{~N} \mathrm{HCl}$. Images of the tissue directly adjacent to the mesh were taken at a magnification of 200x using cross polarization light microscopy (Leica DM5000B, Leica Microsystems ', Switzerland). Per group, seven samples were randomly selected for this analysis. Every sample was analysed three times at random locations along the mesh. Collagen differentiation was measured by calculating the collagen type 1:3 ratio using the Qwin morphometry-system (Leica QWin V3.5.1, Leica Microsystems ${ }^{\circ}$ ) and a customized automated algorithm for computer quantification of the various red and green expression of the picro Sirius red staining.

\section{Neovascularization}

Quantification of vascularization was performed by measuring the mean number of blood vessels in three high power fields, using a magnification of 200x. The analysis was performed by two experienced, independent observers, blinded for sample allocation.

\section{Statistical analysis}

All analyses and graphs were performed/made using GraphPad Prism, version 6.0 for Windows (GraphPad Software ${ }^{\circledR}$, USA). Results are presented as medians with error bars (whiskers) representing the interquartile range. Differences between multiple groups were analysed using one-way ANOVA and individual group comparisons were performed using either student's t-tests in case of parametric distribution or MannWhitney $U$ tests in case of non-parametric distribution. Normality was tested using the F-test. A Bonferroni correction for testing multiple groups was applied, resulting in an alfa smaller than 0.05 depending on the number of comparisons.

\section{RESULTS}

Eighty male Wistar Han ${ }^{\circledR}$ IGS outbred rats, eight weeks of age, weighing 250-350 g, were included in this study. All surgical procedures were performed by two certified (art.9 EAA) researchers (KB, MS) experienced in animal surgery. All animals completed the follow-up without complications. Bodyweight in all groups increased steadily, no statistically nor clinically significant differences in body weight were detected between the groups after 90 days follow-up. 


\section{Mesh shrinkage}

After 28 days of follow-up, the Parietex mesh was the only mesh that did not differ statistically significant in size when compared to baseline. The Prolene ${ }^{\circ}$, Prolene Soft, and UltraPro median mesh size was 90.0\%, 95.0\%, and 97.3\% respectively (figure 2). After 90 days follow-up, these results did not change significantly. The UltraPro mesh shrank significantly less when compared to the Prolene $(p=0.019695 \% \mathrm{Cl}[0.882$ 8.886]) and Prolene Soft meshes ( $p=0.012595 \% \mathrm{Cl}$ [1.137-8.093]). The Parietex showed significantly less shrinkage compared to the Prolene Soft mesh ( $p=0.0012$ 95\% $\mathrm{Cl}[2.760-9.355])$.

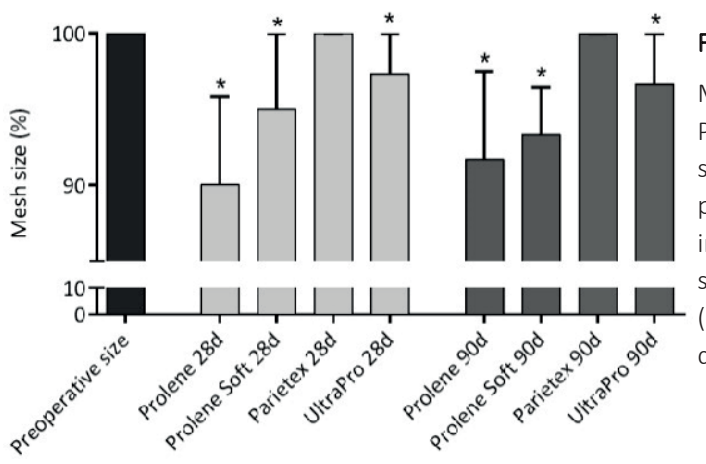

Figure 2 Mesh shrinkage

Median mesh shrinkage after 28 and 90 days. Preoperative mesh size was $3 \times 2 \mathrm{~cm}$, mesh shrinkage is expressed as a percentage compared to the original mesh size. Whiskers indicate the interquartile range. All groups showed parametric distribution of data ( $p=0.4866$ or higher). *Statistically significant difference when compared to baseline.

\section{Mesh ingrowth}

After a follow-up of 28 days the Prolene Soft ${ }^{\circledR}$, Parietex ${ }^{\star}$, and UltraPro ${ }^{\circledR}$ mesh showed comparable tissue ingrowth with a mean tensile strength of $14.0 \mathrm{~N}, 13.6 \mathrm{~N}$, and $15.7 \mathrm{~N}$ required to separate the mesh from the underlying soft tissue (figure 3 ). The Prolene mesh sowed a statistically significant lower ingrowth when compared to the UltraPro

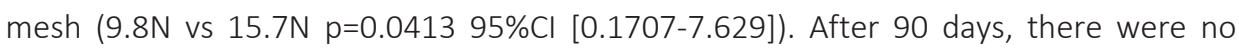
statistically significant differences between the groups (ANOVA $p=0.3653$ ).

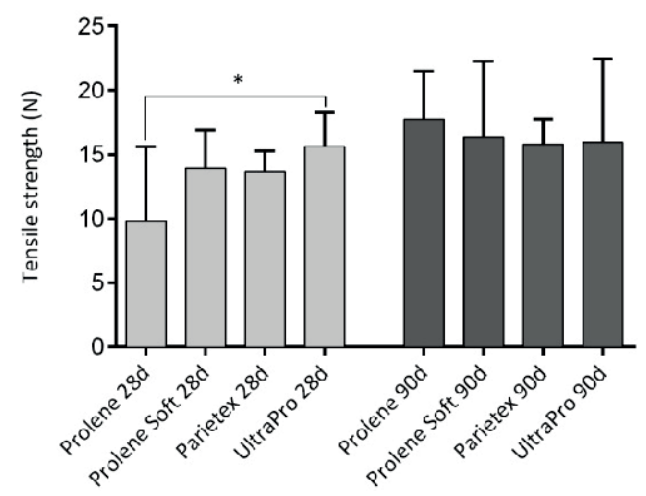

Figure 3 Mesh ingrowth

Mesh ingrowth is defined as the force $(N)$ needed to separate the mesh from the underlying soft tissue. Whiskers indicate interquartile range. Data are distributed parametrically $(p=0.316)$. *Statistically significant difference between connected bars. 


\section{Tissue inflammation}

After 28 days, Parietex mesh showed the highest and Prolene Soft the lowest number of fibroblasts (table 2). The number of granulocytes was comparable between all groups. Overall inflammation was abundantly present in all meshes. Giant cells were seen in moderate to high amounts in Parietex mesh group. At 90 days follow-up, there were no significant difference in the number of fibrotic cells between the groups. Compared to the 28 days measurements, Prolene Soft ${ }^{\circledR}$ meshes showed an increase in inflammation activity and presence of giant cells.

Table 1 Histological evaluation of inflammation

\begin{tabular}{|c|c|c|c|c|c|c|c|}
\hline \multirow[b]{2}{*}{ Mesh } & \multicolumn{4}{|l|}{28 days } & \multicolumn{3}{|l|}{90 days } \\
\hline & Fibrosis & Granulocytes & Inflammation & Giant cells & Fibrosis & Inflammation & Giant cells \\
\hline Prolene & $++/+++$ & $+/-$ & $+/++$ & $+/-$ & ++ & + & $+/-$ \\
\hline $\begin{array}{l}\text { Prolene } \\
\text { Soft }\end{array}$ & $+/++$ & + & + & $+/-$ & ++ & $++/+++$ & ++ \\
\hline Parietex & +++ & $+/-$ & ++ & ++ & $+/++$ & $+/++$ & $+/++$ \\
\hline UltraPro & $++/+++$ & $+/-$ & ++ & $+/-$ & $+/++$ & + & $+/-$ \\
\hline
\end{tabular}

Histological evaluation of different cell types present during microscopically histological evaluation; [-] absent, [+/-] barely present, [+] slightly present, [++] moderately present, [+++] abundantly present.

\section{Collagen formation}

Data were distributed non-parametrically $(p=0.0012)$. After a follow-up period of 28 days, the UltraPro ${ }^{\oplus}$ mesh showed significant higher collagen type 1:3 ratios compared to all other groups $(p<0.0001)$ (figure 4$)$. After 90 days, the collagen $1: 3$ ratio in the UItraPro ${ }^{\circ}$ group declined to a median of 6.4 ( $p<0.0001$ compared to baseline). Prolene and Parietex displayed a statistically significant increase in collagen 1:3 ratio after 90 days follow-up ( $p<0.0001$ compared to baseline). The collagen 1:3 ratio between UItraPro ${ }^{\circ}$ and Prolene Soft $(p=0.5117)$ and Parietex ${ }^{\circ}$ and Prolene $(p=0.9654)$ did not differ statistically significant after 90 days follow-up.

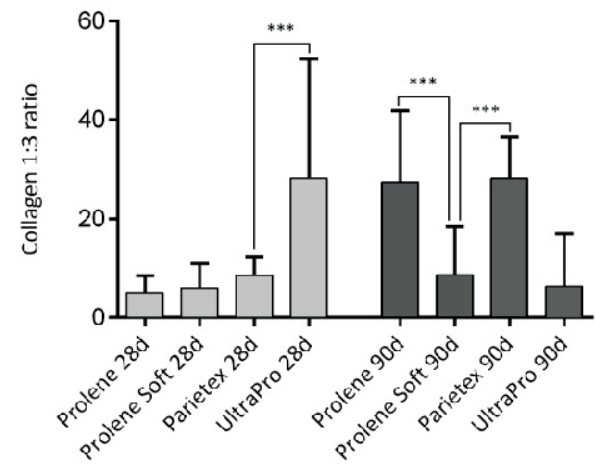

Figure 4 Collagen 1:3 ratio

Ratio of collagen 1:3 measured with computerised algorithm for interpretation of picro Sirius red stain. Whiskers indicate interquartile range. ${ }^{* * *}$ Statistically significant difference $(p<0.001)$. NB: a ratio of ten indicates a tenfold presence of collagen type 1 compared to collagen type 3 . 


\section{Neovascularization}

After 28 days follow-up, the Prolene mesh showed significantly more blood vessel formation when compared to the Prolene Soft ${ }^{\circledR}$ mesh $(P<0.000195 \% \mathrm{Cl}[-15.66$ - -7.804] data not shown). After 90 days follow-up, the initially elevated number of blood vessels in the Prolene mesh group decreased and no statistically significant differences between the groups remained (ANOVA $\mathrm{p}=0.1341$ ), though the Parietex ${ }^{\circledR}$, and Prolene meshes trended towards high blood vessel formation when compared to the UltraPro and Prolene Soft ${ }^{\circ}$ meshes (20 and 16 vessels per HPF vs 13 and 11 vessels per HPF respectively).

\section{DISCUSSION}

This prospective macro- and microscopic evaluation of three monofilament polypropylene meshes, and one multifilament polyester mesh confirmed that pore size, surface modification and mesh weight profoundly influence mesh shrinkage, collagen ratio, and neovascularization.

Shrinkage of a surgical mesh is caused by scar formation around the mesh filaments. Scar tissue can shrink up to $60 \%$ during wound healing. ${ }^{9,13}$ Shrinkage occurs most often in heavyweight meshes with a relatively small pore size, as is confirmed in our series where the heaviest mesh with the smallest pore size (Prolene) showed the most profound shrinkage, and the lightest mesh, with the largest pore size in the polypropylene group (UltraPro ${ }^{\circ}$ ) showed the least percentage of shrinkage. Alongside pore size, mesh material may have an even more pronounced influence on mesh shrinkage as the polyester multifilament mesh (Parietex) showed hardly any shrinkage at all, despite its heavier weight and smaller pore size compared to UltraPro ${ }^{\circ}$. This observation has been confirmed by Bron et al. in their 2010 literature review, and many other studies. ${ }^{9,14,15}$ Brown et al. reports that mesh shrinkage is most pronounced in Prolene meshes (75$94 \%)$ and least in the Ultrapro mesh $(<5 \%)$, polyester meshes were not included in their study. Klinge et al. performed an in vivo study in dogs an observed a comparable shrinkage of 30-50\% in polypropylene based meshes after a follow-up of four weeks. ${ }^{16}$

The lower amount of shrinkage in the polyester mesh could indicate a lower fibrin deposition surrounding the mesh. ${ }^{17}$ This theory is coherent with the observed amount of inflammation and fibrin deposition in the microscopic analysis of the polyester mesh.

Jerabek et al. used several polypropylene based meshes in a subcutaneous rabbit model and described that tissue incorporation is not dependent on pore size, an observation that is confirmed in our series, as we did not observe significant differences in tissue-ingrowth. ${ }^{18}$ However, pore size does have an important role in both foreign body reaction, biocompatibility, and anti-inflammatory characteristics, where large pore meshes seem to induce less foreign body reaction compared to small pore meshes. ${ }^{4,18}$ 
Healthy tissue comprises of approximately 80\% collagen type 1 and 20\% collagen type 3. During the tissue formation phase of normal wound healing, the synthesis of both collagen 1 and 3 will increase, though collagen type 3 increases more than collagen type 1 , resulting in a lower 1:3 ratio. During the remodelling stage of wound healing (weeks to months after injury) this ratio will slowly increase to the original level, whilst collagen type 1 is synthetized. ${ }^{19}$ In our series the Prolene Soft mesh remains a stable, but relatively low, collagen 1:3 ratio throughout the follow-up. The Parietex mesh shows a classic collagen maturation with a lower collagen $1: 3$ ratio at 28 days and a high 1:3 ratio after 90 days follow-up. Remarkably, the UltraPro mesh shows a statistically significant higher collagen 1:3 ratio than any other group after 28 days, though after 90 days collagen $1: 3$ ratios decreased by $78 \%$. This could be explained by an immature collagen deposition throughout the follow-up that might be caused by the degradation of the Monocryl surface modification, which degrades over a 100-day period, though this theory is not supported by the amount of inflammation observed in the Ultrapro group after 90 days. The high collagen ratios of the Parietex and Prolene meshes indicate that a more mature, stronger, and thicker type of collagen fibers was formed at the end of follow-up. A high collagen 1:3 ratio is necessary for strong wound healing. ${ }^{20}$ In 2013 Klosterhalfen et al. analysed collagen 1:3 ratios in explanted meshes. ${ }^{21}$ They described a low collagen 1:3 ratio in the connective tissue surrounding the explanted meshes of patients with a recurrence. Stressing the importance of adequate collagen deposition to ensure a reliable hernia repair, especially in patients with known collagen impairment such as certain types of Ehlers Danlos syndrome, Marfan syndrome, and other collagen disorders. For these specific patients, polyester mesh may be preferred over lightweight polypropylene due to the combination of low shrinkage and high collagen type 1 stimulation in polyester meshes, though more research in this specific population is needed.

This study is limited in the number of mesh comparisons. The placement of the mesh does not reflect the anatomical plane used most frequently in ventral abdominal wall herniorrhaphy, although subcutaneous 'onlay' placement is a common position for mesh reinforcement, the retro-muscular 'sublay' placement is used more frequent, limiting the clinical translation of this study. Despite comparable inflammatory response between the onlay and sublay position according to Binnebösel et al., foreign body reaction, collagen deposition, and mesh ingrowth may vary between different mesh positions. ${ }^{22}$ Despite the abovementioned limitations, the authors believe that the following conclusion can be drawn from this study.

\section{CONCLUSION}

Parietex polyester mesh has hardly any mesh shrinkage when placed in an onlay position and performs significantly better in this outcome than any of the polypropylene 
meshes included in this study. Tissue incorporation was similar for all the included meshes, regardless of mesh weight, pore size or mesh material. Parietex and Prolene both showed high amounts of collagen type 1 deposition around the mesh, indicating that both meshes induce in a layer of healthy, mature collagen deposition without chronic tissue remodelling.

\section{ACKNOWLEDGEMENTS}

The authors would like to thank Dr. Ir. J. Cleutjens for his valuable assistance with the automated digital microscopic analysis of the Picro Sirius red stain.

\section{COMPLIANCE WITH ETHICAL STANDARDS}

All applicable international, national, and/or institutional guidelines for the care and use of animals were followed. All procedures involving animals were in accordance with the ethical standards of the institution or practice at which the studies were conducted.

\section{DISCLOSURES}

Medtronic-Covidien supplied the Parietex mesh for this study free of charge. No financial, or other compensation was received from any party involved in this study, other than the aforementioned meshes. None of the authors have any personal disclosures. 


\section{REFERENCES}

1. Silecchia G, Campanile FC, Sanchez L, et al. Laparoscopic ventral/incisional hernia repair: updated Consensus Development Conference based guidelines [corrected]. Surgical endoscopy 2015;29:2463-84.

2. Usher FC, Ochsner J, Tuttle LL, Jr. Use of marlex mesh in the repair of incisional hernias. The American surgeon 1958;24:969-74.

3. Costello CR, Bachman SL, Grant SA, Cleveland DS, Loy TS, Ramshaw BJ. Characterization of heavyweight and lightweight polypropylene prosthetic mesh explants from a single patient. Surgical innovation 2007;14:168-76.

4. Klinge U, Klosterhalfen B, Birkenhauer V, Junge K, Conze J, Schumpelick V. Impact of polymer pore size on the interface scar formation in a rat model. The Journal of surgical research 2002;103:208-14.

5. Klinge U, Klosterhalfen B, Müller M, Schumpelick V. Foreign body reaction to meshes used for the repair of abdominal wall hernias. European Journal of Surgery 1999;165:665-73.

6. Cobb WS, Kercher KW, Heniford BT. The argument for lightweight polypropylene mesh in hernia repair. Surgical innovation 2005;12:63-9.

7. Klinge $U$, Junge K, Stumpf M, Öttinger AP, Klosterhalfen B. Functional and morphological evaluation of a low-weight, monofilament polypropylene mesh for hernia repair. Journal of Biomedical Materials Research 2002;63:129-36.

8. Klinge $U$, Klosterhalfen $B$. Modified classification of surgical meshes for hernia repair based on the analyses of 1,000 explanted meshes. Hernia : the journal of hernias and abdominal wall surgery 2012;16:251-8.

9. Brown CN, Finch JG. Which mesh for hernia repair? Annals of The Royal College of Surgeons of England 2010;92:272-8.

10. Sheldon HK, Gainsbury ML, Cassidy MR, Chu DI, Stucchi AF, Becker JM. A sprayable hyaluronate/carboxymethylcellulose adhesion barrier exhibits regional adhesion reduction efficacy and does not impair intestinal healing. Journal of gastrointestinal surgery : official journal of the Society for Surgery of the Alimentary Tract 2012;16:325-33.

11. Greca FH, de Paula JB, Biondo-Simoes ML, et al. The influence of differing pore sizes on the biocompatibility of two polypropylene meshes in the repair of abdominal defects. Experimental study in dogs. Hernia : the journal of hernias and abdominal wall surgery 2001;5:59-64.

12. Schreinemacher MH, Emans PJ, Gijbels MJ, Greve JW, Beets GL, Bouvy ND. Degradation of mesh coatings and intraperitoneal adhesion formation in an experimental model. Br J Surg 2009;96:305-13.

13. Garcia-Urena MA, Vega Ruiz V, Diaz Godoy A, et al. Differences in polypropylene shrinkage depending on mesh position in an experimental study. American journal of surgery 2007;193:538-42.

14. Klinge $U$, Klosterhalfen B, Müller M, Öttinger AP, Schumpelick V. Shrinking of Polypropylene Mesh in vivo: An Experimental Study in Dogs. The European Journal of Surgery 1998;164:965-9.

15. Coda A, Bendavid R, Botto-Micca F, Bossotti M, Bona A. Structural alterations of prosthetic meshes in humans. Hernia : the journal of hernias and abdominal wall surgery 2003;7:29-34.

16. Klinge $U$, Klosterhalfen B, Muller M, Ottinger AP, Schumpelick V. Shrinking of polypropylene mesh in vivo: an experimental study in dogs. The European journal of surgery = Acta chirurgica 1998;164:965-9.

17. García-Ureña MÁ, Ruiz VV, Godoy AD, et al. Differences in polypropylene shrinkage depending on mesh position in an experimental study. The American Journal of Surgery 2007;193:538-42.

18. Jerabek J, Novotny T, Vesely K, et al. Evaluation of three purely polypropylene meshes of different pore sizes in an onlay position in a New Zealand white rabbit model. Hernia : the journal of hernias and abdominal wall surgery 2014;18:855-64.

19. Xue M, Jackson CJ. Extracellular Matrix Reorganization During Wound Healing and Its Impact on Abnormal Scarring. Advances in Wound Care 2015;4:119-36.

20. Junge $K$, Klinge $U$, Rosch $R$, et al. Decreased collagen type $\mathrm{I} / \mathrm{III}$ ratio in patients with recurring hernia after implantation of alloplastic prostheses. Langenbeck's archives of surgery 2004;389:17-22. 
21. Klosterhalfen B, Klinge U. Retrieval study at 623 human mesh explants made of polypropylene--impact of mesh class and indication for mesh removal on tissue reaction. Journal of biomedical materials research Part B, Applied biomaterials 2013;101:1393-9.

22. Binnebosel $\mathrm{M}$, Klink CD, Otto J, et al. Impact of mesh positioning on foreign body reaction and collagenous ingrowth in a rabbit model of open incisional hernia repair. Hernia : the journal of hernias and abdominal wall surgery 2010;14:71-7. 



\section{Chapter}

\section{Use of a bio absorbable mesh for complex ventral hernia repair in a contaminated field: \\ a case report}

\section{Elwin H.H. Mommers ${ }^{1}$, Lukas K. van Vugt ${ }^{1 *}$, Nicole D. Bouvy ${ }^{1}$, Johannes A. Wegdam², Tammo S. de Vries Reilingh ${ }^{2}$ \\ ${ }^{1}$ Department of Surgery, Maastricht University Medical Center, Maastricht, The Netherlands ${ }^{2}$ Department of General Surgery, Elkerliek Hospital, Helmond, The Netherlands}

Submitted to 'Hernia' 

Use of a mesh in ventral hernia repair is essential to ensure a durable repair. There are three types of surgical mesh available for ventral herniorrhaphy: synthetic meshes, biologic meshes, and absorbable synthetic meshes. The preferred mesh for application in a (clean-)contaminated field is still under debate. ${ }^{1}$ Some recommend the use of a biological mesh in contaminated circumstances. ${ }^{2}$ Rosen et al. recently demonstrated that absorbable synthetic meshes can form an alternative to biological meshes in (clean-)contaminated ventral herniorrhaphy. ${ }^{3}$ The case presented here describes the formation of an infected seroma on top of a biological synthetic mesh (Phasix ${ }^{\mathrm{TM}}$ ) after placement in a contaminated field.

A 66-year-old female was referred with a recurrent, symptomatic ventral hernia. The patient had an elaborate surgical history that included open gastric bypass surgery, open abdominal wall reconstruction for multiple ventral hernias with intraperitoneal polyester mesh reinforcement, and most recently a spontaneous perforation of the gastrojejunostomy which required reintervention.
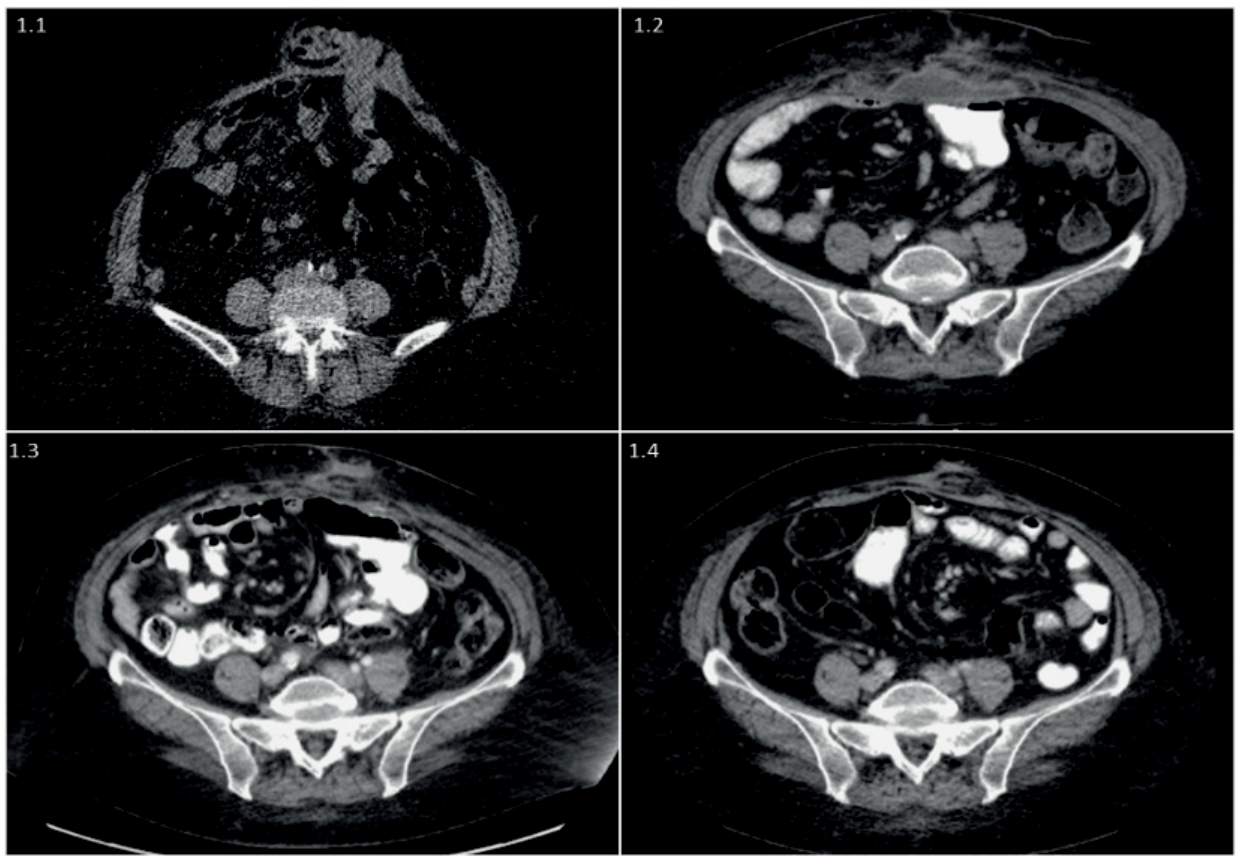

Figure 1 Consecutive CT-scans of the lower abdomen

Four consecutive abdominal CT-scans showing the presence of a recurrent incisional ventral midline hernias before surgery (1.1), a fluid collection on top of the mesh with peripheral wall enhancement seventeen days postoperative, suggestive of an infected seroma (1.2), a residing fluid collection without wall enhancement two months postoperative (1.3) and the near complete resolution of the fluid collection six months postoperative (1.4). 
Physical examination demonstrated intact skin, without signs of infection and multiple reducible hernias in the midline (swiss cheese aspect). Computed tomography (CT) scan demonstrated two groups of hernias in zones M2 and M3 of the European Hernia Society classification for ventral abdominal hernias (figure 1.1).

After a multidisciplinary hernia meeting, it was concluded that the patient suffered from a complex ventral hernia (M3, W3), that could be treated using the endoscopically assisted component separation technique (ECST) with a retromuscular mesh reinforcement.

Before incision, cefotaxime and metronidazole were administered as antibiotic prophylaxis. During the operation, the previously placed intra-abdominal mesh was surrounded by reactive tissue and multiple fluid collections along the perimeter of the mesh, raising the suspicion of a chronic mesh infection. Cultures were obtained. After resection of the mesh, ECST was performed and the posterior rectus fascia was closed. A $35 \times 14 \mathrm{~cm}$ absorbable synthetic mesh (Phasi $\mathrm{X}^{\mathrm{TM}}$ ) was placed on top of the posterior rectus fascia, in a sublay position, and fixated. The anterior rectus fascia, subcutis and skin were closed using absorbable sutures. The patient was discharged at the third postoperative day without complications. No bacteria were cultured in the fluid sample of the removed mesh.

Sixteen days later the patient presented with malaise and fever $\left(38.7^{\circ} \mathrm{C}\right)$. No other clinical signs of wound infection were observed. She didn't report any urogenital complaints and general physical examination failed to identify the cause of her complaints. Both leukocytes (11.9 10^9/L) and C-reactive protein (129 mg/L) were increased. A CTscan of her thorax showed no abnormalities and hence excluded pneumonia, though a CT of the abdomen (figure 1.2) demonstrated an $8 \times 1.5 \mathrm{~cm}$ fluid collection with peripheral enhancement on top of the mesh, which was suggestive of an infection. Following the clinical diagnosis of an infected seroma, cefuroxime 1500 mg was intravenously (IV) administered three times per day for four days. After four days of IV antibiotics the patient's clinical recovery was sufficient to switch to oral antibiotics (amoxicillin/clavulanic acid 500/125 mg three times a day) and continued for one week.

Four weeks after surgery, a small wound dehiscence was noticed leaking serous, and later purulent, discharge from the seroma. CT showed a spontaneous reduction of the fluid collection and decreased inflammatory characteristics (figure 1.3). No therapy was commenced.

A CT-scan, six months postoperative, demonstrated hardly any residual fluid collection and no radiological signs of inflammation (figure 1.4). The patient was discharged from follow-up besides the standard one-year follow-up after complex abdominal wall surgery.

A surgical mesh in ventral hernia repair is not without risk in (clean-)contaminated fields due to the high risk of mesh infection. ${ }^{3,4}$ Bacterial adhesion to the mesh facilitates colonization and subsequent biofilm formation which prevents the immune system from clearing the bacteria ${ }^{4}$. The Phasix ${ }^{\mathrm{TM}}$ mesh is a monofilament, hydrophilic, absorba- 
ble synthetic mesh of poly-4-hydroxybutyrate (P4HB). ${ }^{5,6}$ These characteristics have been shown to negatively affect bacterial adhesion and biofilm formation, because there are less niches where bacteria can adhere and shield from leucocytes. ${ }^{4,7}$ Moreover, hydrophobicity of a mesh is shown to increase slime producing bacterial colonies, which prevents adequate penetration of antibiotics to a bacterial colony. ${ }^{4}$ These mechanisms might have contributed to the successful clearing of the infected seroma. However, theories about the antibacterial properties of $\mathrm{P} 4 \mathrm{HB}$ meshes remain speculative.

In this case, infection of the in situ-mesh was highly plausible due to the perioperative findings, despite the negative bacterial culture. Contamination with intestinal flora following the gastrojejunal perforation is a potential cause of infection of the first mesh. A second limitation is the lack of microbiological confirmation of the infected seroma. The infected seroma was not punctured, because the benefits of identifying a causative pathogen in a recovering patient did not outweigh the risk of introducing bacteria in the seroma. Other diagnoses are possible, e.g. subclinical urinary tract infection, though this does not explain the infective radiological signs of the seroma. The timing of complaints, radiological features (peripheral enhancement, fat stranding), and clinical features (fever, leucocytosis) are very suspicious for an infected seroma or beginning mesh infection. ${ }^{8}$

To our knowledge, this is the first report that describes the successful implantation of an absorbable synthetic P4HB mesh in a contaminated field, most likely complicated by an infected seroma that was treated conservatively. 


\section{REFERENCES}

1. Atema JJ, de Vries FE, Boermeester MA. Systematic review and meta-analysis of the repair of potentially contaminated and contaminated abdominal wall defects. American journal of surgery 2016;212:98295.e1.

2. Breuing K, Butler CE, Ferzoco S, et al. Incisional ventral hernias: Review of the literature and recommendations regarding the grading and technique of repair. Surgery 2010;148:544-58.

3. Rosen MJ, Bauer JJ, Harmaty M, et al. Multicenter, Prospective, Longitudinal Study of the Recurrence, Surgical Site Infection, and Quality of Life After Contaminated Ventral Hernia Repair Using Biosynthetic Absorbable Mesh: The COBRA Study. Annals of Surgery 2017;265:205-11.

4. Engelsman AF, van der Mei HC, Busscher HJ, Ploeg RJ. Morphological aspects of surgical meshes as a risk factor for bacterial colonization. British Journal of Surgery 2008;95:1051-9.

5. Martin DP, Badhwar A, Shah DV, et al. Characterization of poly-4-hydroxybutyrate mesh for hernia repair applications. The Journal of surgical research 2013;184:766-73.

6. Daranarong D, Chan RT, Wanandy NS, Molloy R, Punyodom W, Foster L. Electrospun polyhydroxybutyrate and poly(L-lactide-co-epsilon-caprolactone) composites as nanofibrous scaffolds. BioMed research international 2014;2014:741408.

7. Brown CN, Finch JG. Which mesh for hernia repair? Annals of The Royal College of Surgeons of England 2010;92:272-8.

8. Aguirre DA, Santosa AC, Casola G, Sirlin CB. Abdominal Wall Hernias: Imaging Features, Complications, and Diagnostic Pitfalls at Multi-Detector Row CT. RadioGraphics 2005;25:1501-20. 


\section{Chapter}

\section{The general surgeon's perspective of rectus diastasis}

\section{A systematic review of treatment options}

Elwin H.H. Mommers ${ }^{1}$, Jeroen E.H. Ponten ${ }^{2}$, Aminah K. Al Omar ${ }^{1}$, Tammo S. de Vries Reilingh ${ }^{3}$, Nicole D. Bouvy ${ }^{1}$, Simon W. Nienhuijs ${ }^{2}$

${ }^{1}$ Department of Surgery, Maastricht University Medical Center, Maastricht, The Netherlands

${ }^{2}$ Department of Surgery, Catharina hospital, Eindhoven, The Netherlands

${ }^{3}$ Department of Surgery, Elkerliek hospital, Helmond, The Netherlands

Surgical Endoscopy 2017 May doi:10.1007/s00464-017-5607-9. Epub 2017 June $8^{\text {th }}$ ahead of print 


\section{ABSTRACT}

Background: Diastasis of the rectus abdominis muscles (DRAM) is characterized by thinning and widening of the linea alba, combined with laxity of the ventral abdominal musculature. This causes the midline to "bulge" when intra-abdominal pressure is increased. Plastic surgery treatment for DRAM has been thoroughly evaluated, though general surgical treatments, and the efficacy of physiotherapy remain elusive. The aim of this systematic literature review is to evaluate both general surgical and physiotherapeutic treatment options for restoring DRAM in terms of postoperative complications, patient satisfaction, and recurrence rates.

Method: MEDLINE ${ }^{\circledR}$, Embase, PubMed, PubMed Central ${ }^{\circledR}$, The Cochrane Central Registry of Controlled Trials (CENTRAL), Google Scholar, and the Physiotherapy Evidence Database (PEDro) were searched using the following terms: 'rectus diastasis', 'diastasis recti', 'midline', and 'abdominal wall'. All clinical studies concerning general surgical or physiotherapeutic treatment of DRAM were eligible for inclusion.

Results: Twenty articles describing 1.691 patients (1.591 surgery/ 100 physiotherapy) were included. Surgical interventions were classified as plication techniques (313 patients; 254 open/ 59 laparoscopic), modified hernia repair techniques (68 patients, all open), and combined hernia \& DRAM techniques (1.210 patients; 1.149 open/ 40 hybrid). The overall methodological quality was low. Plication techniques with interrupted sutures and mesh reinforcement were applied most frequently for DRAM repair. Open repairs were performed in $85 \%$ of patients. There was no difference in postoperative complications or recurrence rate after laparoscopic or open procedures, nor between plication versus modified hernia repair techniques. Physiotherapy programs were unable to reduce IRD in a relaxed state. Though, reduction of IRD during muscle contraction was described.

Conclusion: Both plication-based methods, and hernia repair methods are used for DRAM repair. Based on the current literature, no clear distinction in recurrence rate, postoperative complications, nor patient reported outcomes can be made. Complete resolution of DRAM, measured in a relaxed state, following a physiotherapy training programme is not described in current literature. Physiotherapy can achieve a limited reduction in IRD during muscle contraction, the impact of this finding on patient satisfaction, cosmesis, or function outcome is unclear. 


\section{INTRODUCTION}

Diastasis of the rectus abdominis muscles (DRAM) is characterised by a protruding midline following an increase in intra-abdominal pressure. The condition is characterised by a gradual thinning and widening of the linea alba, combined with a general laxity of the ventral abdominal wall muscles. ${ }^{1}$ DRAM is frequently misclassified as a primary ventral hernia, though the musculofascial continuity of the midline and subsequent absence of a true hernia sac is what sets DRAM apart from a ventral hernia. DRAM is defined according to the Beer classification as an inter-rectus distance (IRD) of $22 \mathrm{~mm}$, three centimeters above the umbilicus, measured in a relaxed state. ${ }^{2}$ DRAM occurs most frequently during pregnancy and regresses spontaneously after childbirth in most women. However, 12 months' post-partum, 33\% of women still experience DRAM. ${ }^{3}$

Patients with DRAM can experience similar complaints as patients with ventral hernias, such as lower back pain, functional, and cosmetic impairment, although DRAM doesn't pose any threat of strangulation. ${ }^{4-6}$ DRAM repair is challenging for most general surgeons since guidelines on indication and methods for repair do not exist. The similarity to primary ventral hernias causes frequent misclassification of the disease, and potential mistreatment of DRAM. In recent years, the overall complexity of evidence concerning DRAM treatment has increased. This is due to the development and implementation of several new reconstructive techniques, combined with heterogenous outcome measurements, heterogenous definitions for DRAM, and the lack of high quality data.

DRAM is mostly treated conservatively, with or without the help of a physiotherapist. If conservative therapy is preferred, patients can be referred to a physiotherapist for training programmes that specifically target DRAM, with the aim of reducing IRD and improvement of quality-of-life (QoL). Benjamin et al. evaluated the efficacy of these training programmes in 2014, though due to the low quality of the included studies, no conclusions could be drawn. $^{7}$

In case of severe cosmetic or functional impairment, the patient can be referred to a plastic or general surgeon. Patients that suffer from excess skin or want to tailor their waistline simultaneously with DRAM repair should be referred to a surgeon in the field of plastic and reconstructive surgery for an abdominoplasty. Publications describing DRAM repair in combination with abdominoplasty, liposuction or other strictly plastic surgical techniques are numerous. A recent review of Akram et al. in 2014 concerning abdominoplasty repairs in combination with plication of the linea alba concluded that most evidence is of low quality and RCT's are required to gain more insight in the short and long-term effects of these combined procedures. ${ }^{8}$

Patients with the sole diagnosis of DRAM are frequently referred to the general surgeon. If surgical treatment is considered, several techniques ranging from laparoscopic, endoscopic, hybrid, and open repairs are available. Currently, there is no consensus on the preferred surgical management of DRAM. In contrast to evidence from the reconstructive field, a thorough literature review comparing different surgical techniques for 
DRAM is noticeably absent in general surgery. The aim of this systematic literature review is to provide insight in the general surgical treatment options for DRAM in terms of postoperative complications, patient satisfaction, and recurrence rates, and to evaluate if physiotherapy is an alternative for surgical intervention.

\section{METHODS}

This review was registered on PROSPERO [No.: CRD42016048176] and conducted according to the PRISMA statement. ${ }^{9}$ Before the start of the review process, the review protocol was evaluated and approved by an independent, external expert in the field of ventral hernia repair.

\section{Search strategy}

A structured literature search of MEDLINE ${ }^{\circledR}$, Embase, PubMed, PubMed Central ${ }^{\circledR}(\mathrm{PMC})$, The Cochrane Central Registry of Controlled Trials (CENTRAL), Google Scholar, and the Physiotherapy Evidence Database (PEDro) was performed by two independent reviewers (E.M. and A.A.O.) using the following terms:

'Diastasis recti' OR 'rectus diastasis' OR 'diastasis of the rectus abdominis' OR 'diastasis of the recti' OR 'abdominal diastasis' OR 'abdominal separation' OR 'diastasis recti abdominis' OR 'separation of the recti' OR 'separation of the rectus abdominis' OR 'divarication of the recti' OR 'divarication the rectus abdominis' (See table 1 for PubMed search algorithm).

The last search was performed on the 8th of September 2016. The search was performed using validated methods of the Cochrane collaboration. ${ }^{10}$ Both medical subject heading (MeSH) terms and free-text terms were used to construct the search algorithm. To create a sensitive algorithm, only one domain of search terms (DRAM population) was used. In addition to the above-mentioned database searches all reference lists of included studies were cross-referenced to retrieve additional articles eligible for inclusion. In case of disagreement between the reviewers regarding the eligibility for inclusion of an article, the study quality, or the data abstraction, a third reviewer (NB) was consulted for arbitration. 
Table 1 Search algorithm for PubMed search

((()((()((((diastasis[All Fields] AND recti[All Fields]) OR (rectus[All Fields] AND diastasis[All Fields])) OR (diastasis[All Fields] AND ("rectus abdominis"[MeSH Terms] OR ("rectus"[All Fields] AND "abdominis"[All Fields]) OR "rectus abdominis"[All Fields]))) OR (diastasis[All Fields] AND recti[All Fields])) OR (("abdomen"[MeSH Terms] OR "abdomen"[All Fields] OR "abdominal"[All Fields]) AND diastasis[All Fields])) OR (("abdomen"[MeSH Terms] OR "abdomen"[All Fields] OR "abdominal"[All Fields]) AND ("divorce"[MeSH Terms] OR "divorce"[All Fields] OR "separation"[All Fields]))) OR (diastasis[All Fields] AND recti[All Fields] AND abdominis[All Fields])) OR (("divorce"[MeSH Terms] OR "divorce"[All Fields] OR "separation"[All Fields]) AND recti[All Fields])) OR (("divorce"[MeSH Terms] OR "divorce"[All Fields] OR "separation"[All Fields]) AND ("rectus abdominis"[MeSH Terms] OR ("rectus"[All Fields] AND "abdominis"[All Fields]) OR "rectus abdominis"[All Fields]))) OR (diastasis[All Fields] AND rectus[All Fields] AND abdominus[All Fields])) OR (divarication[All Fields] AND recti[All Fields])) OR (divarication[All Fields] AND rectus[All Fields] AND abdominus[All Fields])) OR (divarication[All Fields] AND ("rectus abdominis"[MeSH Terms] OR ("rectus"[All Fields] AND "abdominis"[All Fields]) OR "rectus abdominis"[All Fields])))

Search algorithm for PudMed database search, performed on 8th of September 2016.

\section{Outcome definition and study selection criteria}

The primary outcome for surgical studies was recurrence rate, secondary outcomes were complication rate within 30 days, and patient satisfaction.

The primary outcome for physiotherapy studies was the effect of the treatment on IRD, secondary outcomes were patient satisfaction, and recurrence rate.

Physiotherapy studies reporting the effect of a single exercise, performed only once, on IRD were considered functional anatomy studies, these studies were not included in this systematic review. Physiotherapy studies focusing on rectus diastasis during pregnancy or during the immediate postpartum period ( $\leq 24$ hours after childbirth) are not included in this review since results obtained during this period cannot be translated to a later time point, and therefore, do not provide an answer to the secondary endpoint of this review.

Any study (comparative, non-comparative, randomized, or observational studies) reporting on a surgical or physiotherapy intervention for DRAM in at least one patients $\geq 18$ years old that reports on the primary and/or secondary outcome were eligible for inclusion in this systematic review, with the exclusion of plastic surgery interventions such as abdominoplasty or liposuction. No limitations to subjects, type of article, or language were applied, though articles published before 1975 which described techniques that have not been applied or described since 1975 were excluded. Articles reporting on the treatment of DRAM combined with primary ventral midline hernias were included yet analysed separately.

\section{Quality assessment}

The quality of randomized controlled trials (RCT's) was evaluated using a 14-item modified Jadad score and the Cochrane risk of bias tool. ${ }^{11,12}$ The quality of non-randomized clinical studies was evaluated using the methodological index for non-randomized stud- 
ies (MINORS) criteria. $^{13}$ In case of missing data for the quality assessment tool or data abstraction the first and corresponding author of the study were contacted via email for completion of the missing data. If the author did not respond, a reminder was sent after two weeks. Studies which scored below three on the MINORS checklist were excluded due to lack of critical data needed for correct interpretation of the results, combined with an unacceptable risk of bias, as at least six of eight domains of the MINORS checklist are either not reported, or partially reported in those publications.

\section{Data abstraction}

Data abstraction was performed in duplicate by two independent reviewers (E.M. and A.A.O.) with a standardized electronic data extraction form including but not limited to the following study variables: title, source, year of publication, study design incl. retrospective or prospective design, demographics of study population, sample size, type of rectus diastasis according to Nahas et al., description of intervention, type of analysis (intention to treat vs per protocol), complications within and after 30 days postoperative, follow-up period, follow-up assessment tool, risk factors for recurrence, and recurrence rate. ${ }^{14}$ The Nahas classification defines four different aesthetic types of DRAM; Type A, DRAM secondary to pregnancy; Type B, DRAM, and laxity of the lateral and infra-umbilical aponeurosis; Type $\mathrm{C}$, congenital lateral insertion of the rectus abdominis muscles; and Type D, DRAM combined with poor waistline.

\section{RESULTS}

After screening 3.689 citations, and removal of 20 duplicate manuscripts, 37 articles were selected for full-text review (Fig. 1). Seventeen articles did not meet the inclusion criteria. Twenty studies describing a total of 1.691 patients, 1.591 (94 males, 1497 females) in general surgical techniques and 100 (all female) in physiotherapy training programmes, were included in this systematic review. Fourteen articles described surgical techniques, and six describing physiotherapy interventions (table 1). Five articles were written Russian, and one in Italian. The included surgical techniques were divided in three categories; 1) plication techniques for DRAM repair, 2) hernia repair techniques modified for DRAM repair, and 3) techniques for DRAM associated with small midline hernias. 


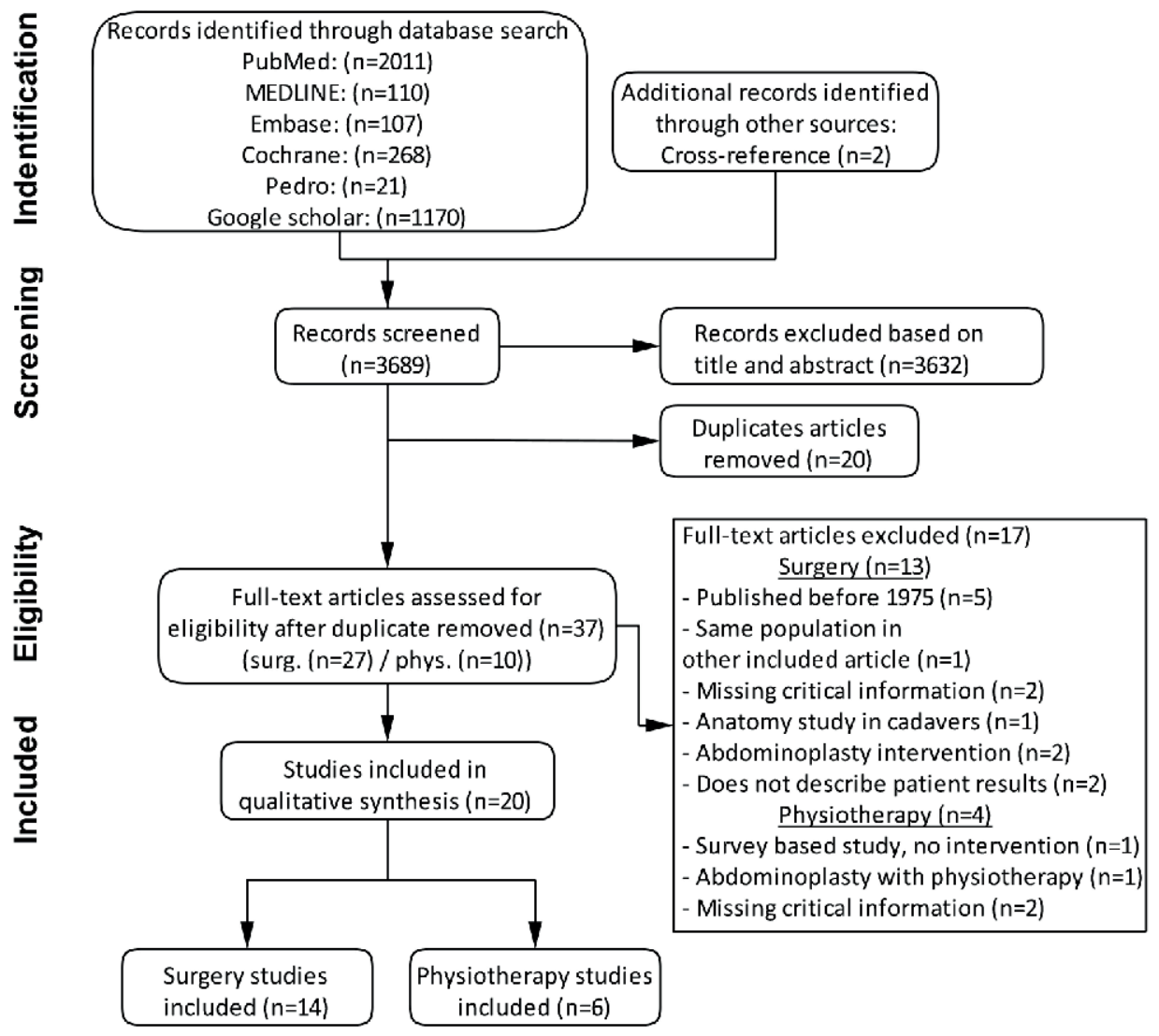

Figure 1 Flow of trials through review

Flow of trials through review PRISMA flowchart of study selection. $n=$ number of studies. This flowchart is in accordance with the 2009 PRISMA statement.

\section{SURGERY}

\section{Plication techniques}

Six retrospective studies described a surgical technique that included plication of the midline, anterior-, or posterior rectus fascia, whilst maintaining the myofascial continuity of the ventral abdominal wall. ${ }^{15-20}$ A total of 313 patients were included in this section. Four studies were case series, and two were case reports (appendix table 2A). Quality of the included studies was low to moderate with MINORS scores ranging from 4 to 7. 


\section{Techniques}

Four studies used a laparoscopic plication technique (Fig. 2A). All laparoscopic studies used mesh reinforcement, of which three used interrupted sutures, and one used a continuous suture. The study of Palanivelu et al. describes a technique in which they place interrupted sutures which run in and out of the widened linea alba several times, causing the midline to fold like a 'Venetian blind' when the sutures are tied. ${ }^{19}$ The difference in suture technique did not lead to a difference in recurrence rate, which were $0 \%$ in all the laparoscopic repair groups after a follow-up ranging from 6 to 24 months.

Three studies used an open technique, and all plicated a different layer of the ventral abdominal wall (Fig. 2B). ${ }^{16-18}$ The study of Nahas et al. included two female patients with a recurrent DRAM and is the only study in this section that did not use mesh reinforcement. They describe two type $C$ (congenital lateral implantation of the rectus abdominis muscle) DRAM patients that had undergone plication of the anterior rectus fascia during abdominoplasty surgery and were operated using an open plication technique of the posterior rectus fascia. ${ }^{18}$ The anterior rectus fascia was then sutured to the midline, to mimic the anatomic situation. The other two 'open' studies described either a plication technique of the anterior rectus fascia or solely the widened linea alba, combined with a sublay mesh.

\section{Results of plication techniques}

Postoperative complications were reported in five studies. Three studies observed postoperative pain, two of which report an incidence of around 10\%. The study of Sahoo et al. clearly shows a surplus of soft tissue clustered at the midline as a direct result of the laparoscopic plication. ${ }^{15}$ One of six studies reported a recurrence after open plication. The original article does not describe recurrences, instead they describe that three patients experienced laxity of the abdominal wall with protrusion, forcing them to wear an abdominal binder. These patients were interpreted as having a recurrent DRAM.

\section{Modified hernia repair techniques}

Two articles used a (modified) hernia repair technique in DRAM patients (appendix table 2A). ${ }^{21,22}$ The first study from Angio et al. included twelve patients with a midline diastasis and performed a modified Chevrel technique (Fig. 2C). ${ }^{21}$ The modified technique leaves the musculofascial continuity of the ventral abdominal wall intact as the abdominal cavity nor the posterior rectus fascia is opened. Instead, the anterior rectus fascia is incised and overturned to form a wider new posterior rectus fascia. The wident midline is not incised. 


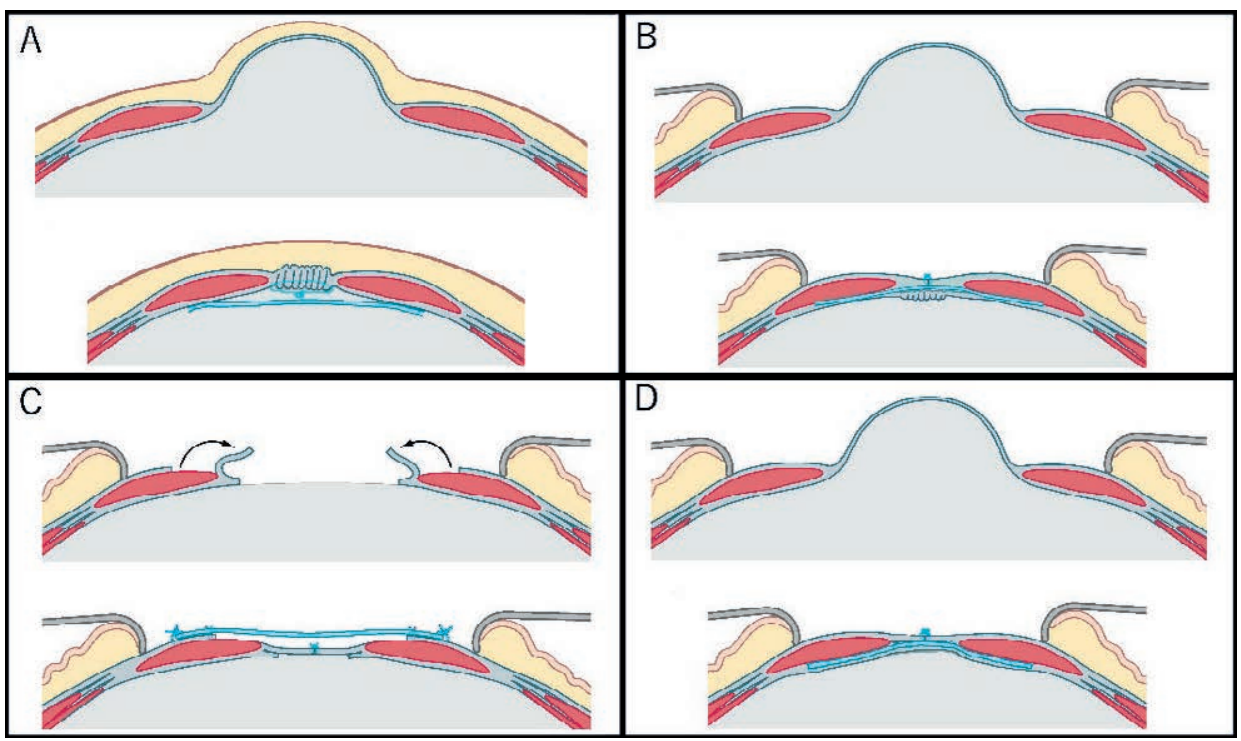

Figure 2 Illustration of surgical interventions

Four main surgical interventions for treating DRAM. $A=$ laparoscopic plication of the entire midline with mesh reinforcement, performed in 59 patients; $B=$ open plication of the posterior rectus fascia, performed in 254 patients; $C=$ modified Chevrel repair, performed in 52 patients; $D=$ Rives-Stoppa like repair with or without mesh reinforcement, performed in 948 patients.

The second study of Gireev et al. described a meshless modification of the Rives-Stoppa repair in which the hernia sac is excised and the posterior rectus fascia is sutured using an overlapping plasty. The anterior rectus fascia is then closed. ${ }^{22}$ Quality of both studies was 8/16 and 3/16 respectively. The population described by Gireev et al. in the 1997 publication was identical to the population in two prior publications of their group, from 1983 and 1994. ${ }^{23,24}$ These three publications were combined in one reference and only included once in this review.

\section{Results of hernia repair techniques}

The study of Angio et al. described seroma formation in $7 \%$ of their population. The study of Gireev et al. only reported nine short term complications, though did not specify these complications in their manuscripts. ${ }^{22}$ Both studies had a follow-up of 24 months and observed no recurrences.

\section{Techniques for combined repair of DRAM and small midline hernia}

Six studies, of which five retrospective case series and one prospective cohort study, described surgical treatments for DRAM associated with small midline hernias (appen- 
dix table 2A). ${ }^{6,25-29}$ The total number of patients in this section was 1.210. Quality of the included studies was low to moderate as one studies scored 11/16 and the remaining MINORS scores ranged from 3 to 5 . Two studies included both umbilical and epigastric hernias. Four studies included small umbilical hernias. Hernia repair in five out of six studies consisted of suture closure of the hernia sac combined with mesh reinforcement. The oldest study (1990), of Ranney et al., describe a modified Rives-Stoppa repair without mesh reinforcement. Four studies described open techniques, mostly resembling either modified Chevrel or Rives-Stoppa procedures (Fig. 2C, D respectively). One described an endoscopic procedure in the anatomical plane between the subcutaneous fat and the anterior rectus fascia, with plication of the midline and onlay mesh reinforcement. One described a hybrid version in the same plane, which resembled a modified Chevrel repair, performed partially endoscopic, with onlay mesh reinforcement.

\section{Results of combined repair DRAM and midline hernia}

Five of the six studies reported on postoperative complications. ${ }^{6,25-28}$ Two of these studies encountered no postoperative complications, and the remaining three encountered only minor (Clavien-Dindo I-II) postoperative complications. Köckerling et al. reported a seroma incidence of $2.5 \%$, and Bellido et al. reported a seroma incidence of $23 \%$, though these patients were initially not offered an abdominal binding after a subcutaneous procedure, which could explain the high incidence of seromas. ${ }^{6}$

Follow-up was only reported in two studies and ranged from 20 months to 14.8 years. Only one recurrence was observed during the follow-up in the study of Privett et al. in a patient with a combined DRAM and umbilical hernia. ${ }^{28}$ They mentioned that that open repair with preperitoneal placement of mesh without approximation of the rectus fascia (resembling a bridged repair) leads to fluctuating cosmetic results, since protrusion of DRAM may still be present after mesh placement.

\section{PHYSIOTHERAPY}

Six studies evaluated the effect of a physiotherapy intervention on IRD in a total of one hundred postpartum women. Two RCT's, two prospective uncontrolled trials, and two case reports were included in this section (appendix table 2B). ${ }^{5,30-34}$ All studies focused on females at different postpartum intervals, ranging from one months to three years. Scientific quality of the included studies was moderate with a MINORS score ranging from six to nine for the non-randomized studies, and a Jadad score of 7 to 11 for the included RCT's. See table 3 for results of The Cochrane risk of bias tool. 


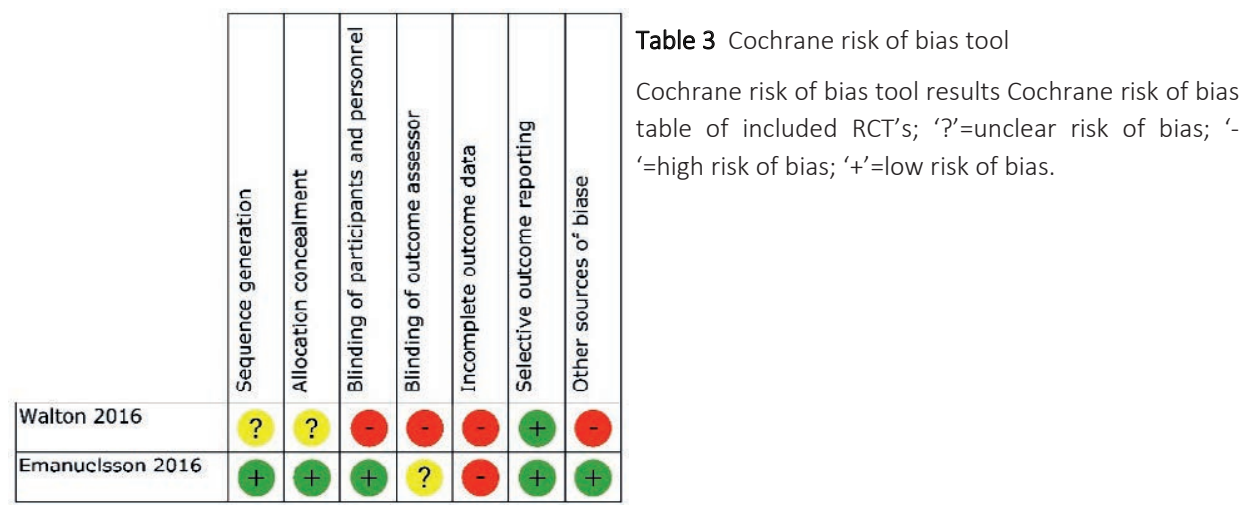

Training programme

One case study used a single exercise (prone kneeling) to train the patients. ${ }^{33}$ Two studies used general (not further specified) strengthening exercises for the abdominal wall, hip, and trunk muscles. ${ }^{5,32}$ The remaining three studies used the head lift exercise, combined with pelvic lock or pelvic tilt exercises. ${ }^{30,31,34}$

Frequency of the training programme varied between the studies. One study let patients train on their own and the frequency of the exercise during the training programme is not reported. ${ }^{33}$ The remaining studies used counselling of a physiotherapist to train patients in allocated training sessions. Frequency of the programmes varies from one time per week, to five times per week. The total number of sessions patients had to participate ranged from 4 to 40 sessions.

\section{Outcome measurement}

Five out of six studies included IRD as an outcome parameter. IRD was measured with a total of four different instruments (ultrasound, tape measure, palpation, and dial caliper). Three studies assessed IRD with two instruments simultaneous. ${ }^{31,32,34}$ Overall, three studies used palpation as measurement for IRD, two studies combined palpation with tape measurement, or dial caliper measurement. One study used only tape measurement ${ }^{33}$, and one used only palpation. ${ }^{30}$ Two studies did not report if the IRD measurement took please during muscle contraction or relaxation. The remaining three studies reporting on IRD, all measured IRD during muscle contraction. Four of the five studies measuring IRD reported the location of IRD measurement. One studies measured at the umbilicus, two studies measured two centimeters above and below the umbilicus and one measured four-and-a-half centimeters above and below the umbilicus. 


\section{Results of physiotherapy training programme}

Five out of six studies reported IRD as outcome measurement. Follow-up in all studies was performed directly after the training programmes ended. Hence, follow-up ranged from 2 weeks to 4 months. Five articles reported IRD as their primary outcome and all reported a decrease of IRD during the exercise programme. Both case reports describe IRD values below $22 \mathrm{~mm}$ during muscle contraction, after completion of the training programme.

The article of Emanuelsson et al. measured QoL using the SF-36 questionnaire and report that $87 \%(n=26)$ of the patients were unsatisfied with the results of their training therapy and opted for surgical intervention after completion of the training programme.

\section{DISCUSSION}

Diastasis of the rectus abdominis muscles is a common problem. If not treated as part of an abdominoplasty in the field of plastic and reconstructive surgery, there is a lack of consensus on the preferred treatment of DRAM. Apart from conservative therapy, operative intervention and physiotherapy are the most frequently reported interventions for DRAM. This review provides an overview of the results of these interventions.

The overall quality of the included studies was low to moderate, combined with limited scientific power since only five prospective studies were included, of which two were RCT's. For this reason, meta-analysis of the included studies was deemed unfit.

As stipulated by the review of Hickey et al. in 2011, indication for DRAM repair has different considerations compared to ventral hernia repair, despite the clinical similarity between the two entities. ${ }^{35}$ Indication for DRAM repair is most often based on cosmetic or functional impairment, as DRAM poses no risk of strangulation. Therefore, the cosmetic results of a surgical technique or physiotherapy training programme, along with other patient reported outcomes (PROs) should be an important outcome of scientific studies. Remarkably, cosmetic outcome was only included in one study, and measured subjectively with an instrument that was not validated. Other PROs were not measured in surgical publications and only reported twice in physiotherapy studies.

\section{Surgical technique}

Based on the published literature, the surgical techniques available for DRAM repair are either plication based or hernia repair based. The plication-based techniques include open plication, laparoscopic plication, or hybrid plication of either the anterior or posterior rectus fascia. Based on the results of this review, there is no clear difference in postoperative complication between these methods. Nearly all studies that described a plication technique used interrupted sutures and mesh reinforcement, which could 
account for the low recurrence rates, though comparative data is not available. The plication techniques can leave a surplus of skin directly after surgery, as described in the study of Sahoo et al., though due to the lack of cosmetic outcome measurement no evidence-based statements regarding the cosmetic postoperative appearance can be made. $^{15}$

Hernia repair techniques can be used for DRAM treatment. The musculofascial continuity of the ventral abdominal wall is an important anatomical structure to considered during DRAM repair. If the midline is incised and the continuity is disturbed, the risk of incisional hernia formation and subsequent risk of strangulation will become larger, though alignment of the rectus muscles could be easier. The current evidence is of insufficient quality to detect differences between techniques that preserve the musculofascial continuity versus techniques that incise the midline. Hernia based techniques for DRAM repair are often modifications of the original Chevrel or Rives-Stoppa techniques. ${ }^{21,36,37}$

An important reason for DRAM patients to seek medical attention is the cosmetic impairment they experience. Despite the importance of cosmetic results, $85 \%$ of patients described in published literature concerning general surgery for DRAM, are operated on via open procedures. Recently developed hybrid techniques such as the ELARplus described by Köckerling et al., the endoscopic midline plication by Bellido et al., or the eMILOS by Reinpold et al. could increase the number of minimally invasive procedures in the DRAM population., ${ }^{6,26,38}$ These procedures are promising variations of classic (open) hernia repair techniques that respect the anatomical myofascial continuity of the ventral abdominal wall, and leave only minimal scarring, without risk of incisional hernia formation because the abdominal cavity is not opened. Given the recent invention of these techniques, the amount of published data is limited and with short followup. The study of Reinpold et al. unfortunately had to be excluded since the article did not describe critical data about the study methodology nor the population. ${ }^{38}$ Nevertheless, the technique seems to be promising for DRAM treatment.

It was not possible to isolate any difference in outcome between male and female patients due to quality and reporting limitations of the included studies. It is the author's opinion that the pathophysiology between males and females, or between type $A / B$, and type C/D DRAM is different. The A/B type DRAM may be based on a physiological response during pregnancy, when collagen is remodelled under the influence of the hormone 'relaxin' to allow widening or stretching of the midline that is not corrected properly after pregnancy. ${ }^{39,40}$ In males, or type C/D DRAM, genetic predisposition or altered collagen 1:3 ratio's may have a more pronounced role.

\section{Physiotherapy}

The literature regarding physiotherapy interventions is heterogeneous in nature and of low quality. The type of exercises used to reduce IRD, the frequency of the exercises, 
the total number of sessions within a training programme, and the instruments used to asses IRD vary greatly amongst the included studies. For instance, the case study of Litos et al. informed their patient to avoid abdominal exercises that could increase IRD by recruitment of the transverse abdominal muscles, such as sit-ups, crunches, and rotational trunk exercises, whilst other studies from Ramesh et al. and Walton et al. target specifically the transverse abdominal muscles with these exercises to reduce IRD..$^{31,32,34}$ The included studies only report on postpartum women (type A, B), making translation of the results to men and type $C$, and D DRAM difficult, if not impossible.

Brauman et al. has investigated the clinical anatomy of DRAM and reported that DRAM is not only associated with a gradual thinning and stretching of the linea alba, but also by a laxity of the ventral abdominal musculature. ${ }^{1}$ Considering Brauman's findings, physiotherapy could play a role in treating the laxity of the ventral abdominal musculature.

Despite the potential benefits of physiotherapy, current literature does not describe the successful treatment of rectus diastasis nor a reduction of IRD measured in a relaxed state. Since diastasis rectus is defined as a separation of the rectus abdominis muscles as measured in a relaxed state we must conclude that the currently available evidence does not describe the successful treatment of rectus diastasis after a physiotherapy training programme. Physiotherapy could moderately reduce IRD during muscle contraction. The impact of these results on quality-of-life or function outcome is currently unknown, as is the sustainability of these results after a follow-up exceeding four months. Based on the study of Emanuelsson et al. physiotherapy alone is unlikely to lead to satisfying functional and cosmetic results. ${ }^{5}$ Emanuelsson et al. compared DRAM patients treated with a physiotherapy training programme with patients whom received abdominoplasty for DRAM in a randomized controlled trial. Eighty-one percent of the patients in the training group were unsatisfied with their functional and cosmetic appearance at the end of the programme and opted for surgical intervention after the trial ended.

Physiotherapy could be an alternative to surgery for patients who are unable or reluctant to undergo surgical intervention. Surgical treatment only corrects the widening of the linea alba and won't influence the general laxity of the ventral abdominal wall. Therefore, physiotherapy could be a useful addition to surgical intervention for DRAM, to achieve satisfying functional outcome.

\section{limitations}

Most of the included studies were performed in postpartum women (rectus diastasis type $A$ and $B$ ), reducing the translatability to men and rectus diastasis type $C$ and $D$. Most of the included studies were retrospective and non-comparative in nature, reducing the scientific power of the review. The quality of the included studies according to the MINORS criteria was low, this is in part due to the 'how-to-do-it' type of publication 
which describes surgical techniques in scientific journals, these articles only include a small and limiting the description of the randomization, inclusion, outcome, and blinding methods. Moreover, the MINORS score is sensitive for retrospective studies, as a retrospective study will automatically lose four out of sixteen points. Due to the low quality of the included studies, gender differences could not be isolated from the included population. Despite the above-mentioned limitations some general recommendations and conclusions can be drawn from this review.

\section{Considerations for DRAM treatment}

\section{'DRAM is not a hernia'}

The continuity of the myofascial anatomy in the ventral abdominal wall is what sets DRAM apart from an abdominal wall hernia. There are endoscopic, hybrid, and open techniques available that leave the anatomical myofascial continuity intact and could potentially protect the DRAM patient from the risk of incisional hernia formation in case of a failed repair. Whether these techniques have any cosmetic or functional advantage over traditional hernia repair techniques, and if there is indeed no risk of incisional hernia formation, is currently undetermined.

\section{'Align and use mesh'}

For the repair of DRAM (without midline hernia), plication techniques with mesh reinforcement and interrupted sutures are most frequently used reconstruct the ventral abdominal wall. Only using mesh reinforcement without an approximation of the rectus fascia of some sort, may lead to unsatisfying cosmetic results. Other minimally invasive, hybrid, or open techniques are promising and can be used, though long-term results and comparative, controlled data are not available.

\section{'Evaluate what's important for the patient'}

The current body of evidence focuses primarily on recurrence rates. It is well known that the risk of recurrence is not the most important variable for the patient. Instead, PROs such as postoperative pain, cosmetic outcome, and functional result are variables that directly concern the patients' wellbeing. The use of PROs is low in both hernia and DRAM related research and should be increased during the coming years. ${ }^{41}$

\section{'Cosmetic outcome is important'}

Cosmetic impairment is an important factor for DRAM patients to seek medical attention. Therefore, cosmetic result of a surgical intervention is of high importance in the DRAM population. Which surgical procedure (endoscopic, hybrid, laparoscopic, or open) has the most satisfying cosmetic outcome is not yet evaluated. 


\section{'Consider physiotherapy'}

Physiotherapy is unlikely to completely treat DRAM, since cases in which IRD was reduced to normal during a relaxed state are currently not described in literature. However, a moderate reduction in IRD during muscle contraction is reported. Whether this has an influence on functional outcomes or quality-of-life is not described. Physiotherapy combined with surgery could potentially have favourable results over surgery alone, this combination of treatments is currently not investigated. Moreover, patients that are reluctant or unable to undergo surgery may be referred to a physiotherapist for strengthening exercises of the abdominal wall.

\section{Recommendations for future studies}

The authors would like to recommend future studies reporting on the efficacy of any DRAM repair to include PROs such as cosmetic outcome, quality-of-life, and work impairment, measured with a validated questionnaire (EuraHS QoL, COMI-Hernia) as their primary outcome, and IRD or recurrence rate as a secondary outcome. Both should be measured using an objective tool (dial caliper or ultrasound), as finger palpation should not be used for scientific outcome reporting. ${ }^{42}$ The location of measurement and the cut-of value should be standardised for patients' age, location of measurement, and pre- or postpartum status according to previously published classifications. ${ }^{2,42-44}$ IRD should be measured whilst the rectus abdominis muscles are relaxed, as references values of normal midline width and DRAM classification are measured/based on measurements during muscle relaxation. Based on the pathophysiology described by Brauman, the combination of physiotherapy and surgical repair has great theoretical potential to solve both the anatomical divarication and the laxity of the ventral abdominal muscles. ${ }^{1}$ The authors recommend that future RCT's focus on the combination of surgery and physiotherapy for the repair of DRAM.

\section{CONCLUSION}

Published literature on surgical treatments for rectus diastasis is of low scientific and methodological quality. Both plication-based methods, as hernia repair methods are used for DRAM repair. Based on the current literature, no clear distinction in recurrence rate, postoperative complications, or patient reported outcomes can be made. DRAM is most frequently repaired using plication techniques combined with mesh reinforcement. Current literature does not describe the successful treatment of DRAM nor a reduction of IRD in a relaxed state following physiotherapy. Physiotherapy can achieve a moderate reduction in IRD during muscle contraction, though it is currently unclear if this has any positive effect on quality-of-life or functional outcomes. 


\section{ACKNOWLEDGEMENTS}

The authors would like to thank Prof. Dr. G. Beets of the Antoni van Leeuwenhoek Hospital, Amsterdam, The Netherlands, for his time and effort associated with reviewing the study protocol in his role as independent, external, expert reviewer.

\section{DISCLOSURES}

Drs. E.H.H. Mommers, Drs. J.E.H. Ponten, Ms. A.K. Al-Omar, Dr. T.S. de Vries Reilingh, Prof. Dr. N.D. Bouvy, and Dr. S.W. Nienhuijs have no conflict of interest or financial ties to disclose directly or indirectly related to this manuscript. 


\section{REFERENCES}

1. Brauman D. Diastasis recti: clinical anatomy. Plastic and reconstructive surgery 2008;122:1564-9.

2. Beer GM, Schuster A, Seifert B, Manestar M, Mihic-Probst D, Weber SA. The normal width of the linea alba in nulliparous women. Clinical anatomy (New York, NY) 2009;22:706-11.

3. Sperstad JB, Tennfjord MK, Hilde G, Ellstrom-Engh M, Bo K. Diastasis recti abdominis during pregnancy and 12 months after childbirth: prevalence, risk factors and report of lumbopelvic pain. British journal of sports medicine 2016;50:1092-6.

4. Parker MA, Millar LA, Dugan SA. Diastasis Rectus Abdominis and Lumbo-Pelvic Pain and Dysfunction-Are They Related? Journal of Women's Health Physical Therapy 2009;33:15-22.

5. Emanuelsson P, Gunnarsson U, Dahlstrand U, Strigard K, Stark B. Operative correction of abdominal rectus diastasis (ARD) reduces pain and improves abdominal wall muscle strength: A randomized, prospective trial comparing retromuscular mesh repair to double-row, self-retaining sutures. Surgery 2016.

6. Bellido Luque J, Bellido Luque A, Valdivia J, et al. Totally endoscopic surgery on diastasis recti associated with midline hernias. The advantages of a minimally invasive approach. Prospective cohort study. Hernia : the journal of hernias and abdominal wall surgery 2015;19:493-501.

7. Benjamin DR, van de Water AT, Peiris CL. Effects of exercise on diastasis of the rectus abdominis muscle in the antenatal and postnatal periods: a systematic review. Physiotherapy 2014;100:1-8.

8. Akram J, Matzen SH. Rectus abdominis diastasis. Journal of plastic surgery and hand surgery 2014;48:163-9.

9. Moher D, Liberati A, Tetzlaff J, Altman DG. Preferred reporting items for systematic reviews and metaanalyses: the PRISMA statement. PLoS medicine 2009;6:e1000097.

10. Higgins JPT GS. Cochrane Handbook for Systematic Reviews of Interventions: The Cochrane Collaboration; 2011.

11. Jadad AR, Moore RA, Carroll D, et al. Assessing the quality of reports of randomized clinical trials: Is blinding necessary? Controlled Clinical Trials 1996;17:1-12.

12. Higgins JP, Altman DG, Gotzsche PC, et al. The Cochrane Collaboration's tool for assessing risk of bias in randomised trials. BMJ (Clinical research ed) 2011;343:d5928.

13. Slim K, Nini E, Forestier D, Kwiatkowski F, Panis Y, Chipponi J. Methodological index for non-randomized studies (minors): development and validation of a new instrument. ANZ journal of surgery 2003;73:712-6.

14. Nahas FX. An aesthetic classification of the abdomen based on the myoaponeurotic layer. Plastic and reconstructive surgery 2001;108:1787-95; discussion 96-7.

15. Kumar AT, Sahoo MR. Laparoscopic plication and mesh repair for diastasis recti: A case series. International Journal of Case Reports and Images 2014;5:610.

16. Deriugina MS. [A method of surgical treatment of diastasis recti abdominis]. Vestnik khirurgii imeni I । Grekova 2001;160:77-8.

17. Shirah BHS, H.A. The Effectiveness of Polypropylene Mesh in the Open and Laparoscopic Repair of Divarication of the Recti. Med Imp Surg 2016; Volume 1.

18. Nahas FX, Ferreira LM, Mendes Jde A. An efficient way to correct recurrent rectus diastasis. Aesthetic plastic surgery 2004;28:189-96.

19. Palanivelu C, Rangarajan M, Jategaonkar PA, Amar V, Gokul KS, Srikanth B. Laparoscopic repair of diastasis recti using the 'Venetian blinds' technique of plication with prosthetic reinforcement: a retrospective study. Hernia : the journal of hernias and abdominal wall surgery 2009;13:287-92.

20. Siddiky AH, Kapadia CR. Laparoscopic plication of the linea alba as a repair for diastasis recti - a mesh free approach. Journal of surgical case reports 2010;2010:3.

21. Angio LG, Piazzese $E$, Pacile $V$, et al. [The surgical treatment of the diastasis recti abdominis: an original technique of prosthesis repair of the abdominal wall]. II Giornale di chirurgia 2007;28:187-98.

22. Gireev GI, Zagirov UZ, Shakhnazarov AM. [Treatment of linea alba hernia and diastasis of rectus abdominis]. Khirurgiia 1997:58-61. 
23. Gireev GIG. [The pathogenesis and surgical treatment of diastasis recti abdominis]. Vestnik khirurgii imeni II Grekova;148:372-5.

24. Zagirov UZ, Gireev GI, Shakhnazarov AM. [Results of surgical treatment of diastasis of the rectus abdominis muscles]. Khirurgiia 1994:24-7.

25. Aiekseev AVYLAADNKMVIAK. Short-term surgical treatment outcomes of patients with combined umbilical hernia and diastasis recti. Moscal Surgical Journal 2014;5:4.

26. Kockerling F, Botsinis MD, Rohde C, Reinpold W. Endoscopic-Assisted Linea Alba Reconstruction plus Mesh Augmentation for Treatment of Umbilical and/or Epigastric Hernias and Rectus Abdominis Diastasis - Early Results. Frontiers in surgery 2016;3:27.

27. Matei OA, Runkel N. A novel technique of midline mesh repair for umbilical hernia associated with diastasis recti. Surgical technology international 2014;24:183-7.

28. Privett BJ, Ghusn M. Proposed technique for open repair of a small umbilical hernia and rectus divarication with self-gripping mesh. Hernia : the journal of hernias and abdominal wall surgery 2016;20:527-30.

29. Ranney B. Diastasis recti and umbilical hernia causes, recognition and repair. South Dakota journal of medicine 1990;43:5-8.

30. Acharry N, Kutty RK. Abdominal Exercise with Bracing, a Therapeutic Efficacy in Reducing Diastasis-Recti among Postpartal Females. International Journal of Physiotherapy and Research 2015;3:999-1005.

31. Kh SR, ale, H D. Effects of Abdominal Exercises on Reduction of Diastasis Recti in Postnatal Women. IJHSR 2016;6:182-91.

32. Litos K. Progressive Therapeutic Exercise Program for Successful Treatment of a Postpartum Woman With a Severe Diastasis Recti Abdominis. Journal of Women's Health Physical Therapy 2014;38:58-73.

33. Sheppard S. The Role of Transversus Abdominus in Post Partum Correction of Gross Divarication Recti. Manual therapy 1996;1:214-6.

34. Walton LM, Costa A, LaVanture D, Mcllrath S, Stebbins B. The effects of a 6 week dynamic core stability plank exercise program compared to a traditional supine core stability strengthening program on diastasis recti abdominis closure, pain, oswestry disability index (ODI) and pelvic floor disability index scores (PFDI). Physical Therapy and Rehabilitation 2016;3:3.

35. Hickey F, Finch JG, Khanna A. A systematic review on the outcomes of correction of diastasis of the recti. Hernia : the journal of hernias and abdominal wall surgery 2011;15:607-14.

36. Stoppa RE. The treatment of complicated groin and incisional hernias. World J Surg 1989;13:545-54.

37. Chevrel JP. [The treatment of large midline incisional hernias by "overcoat" plasty and prothesis (author's transl)]. La Nouvelle presse medicale 1979;8:695-6.

38. Reinpold. Endoscopic assisted mini-open transhernial sublay repair (eMILOS) of symptomatic diastasis recti. Hernia : the journal of hernias and abdominal wall surgery 2016;20.

39. Samuel CS, Lekgabe ED, Mookerjee I. The effects of relaxin on extracellular matrix remodeling in health and fibrotic disease. Advances in experimental medicine and biology 2007;612:88-103.

40. MacLennan AH. Relaxin--a review. The Australian \& New Zealand journal of obstetrics \& gynaecology 1981;21:195-202.

41. Antonescu I, Mueller CL, Fried GM, Vassiliou MC, Mayo NE, Feldman LS. Outcomes reported in highimpact surgical journals. The British journal of surgery 2014;101:582-9.

42. van de Water AT, Benjamin DR. Measurement methods to assess diastasis of the rectus abdominis muscle (DRAM): A systematic review of their measurement properties and meta-analytic reliability generalisation. Manual therapy 2016;21:41-53

43. Coldron Y, Stokes MJ, Newham DJ, Cook K. Postpartum characteristics of rectus abdominis on ultrasound imaging. Manual therapy 2008;13:112-21.

44. van de Water ATM, Benjamin DR. Measurement methods to assess diastasis of the rectus abdominis muscle (DRAM): A systematic review of their measurement properties and meta-analytic reliability generalisation. Manual therapy 2016;21:41-53. 


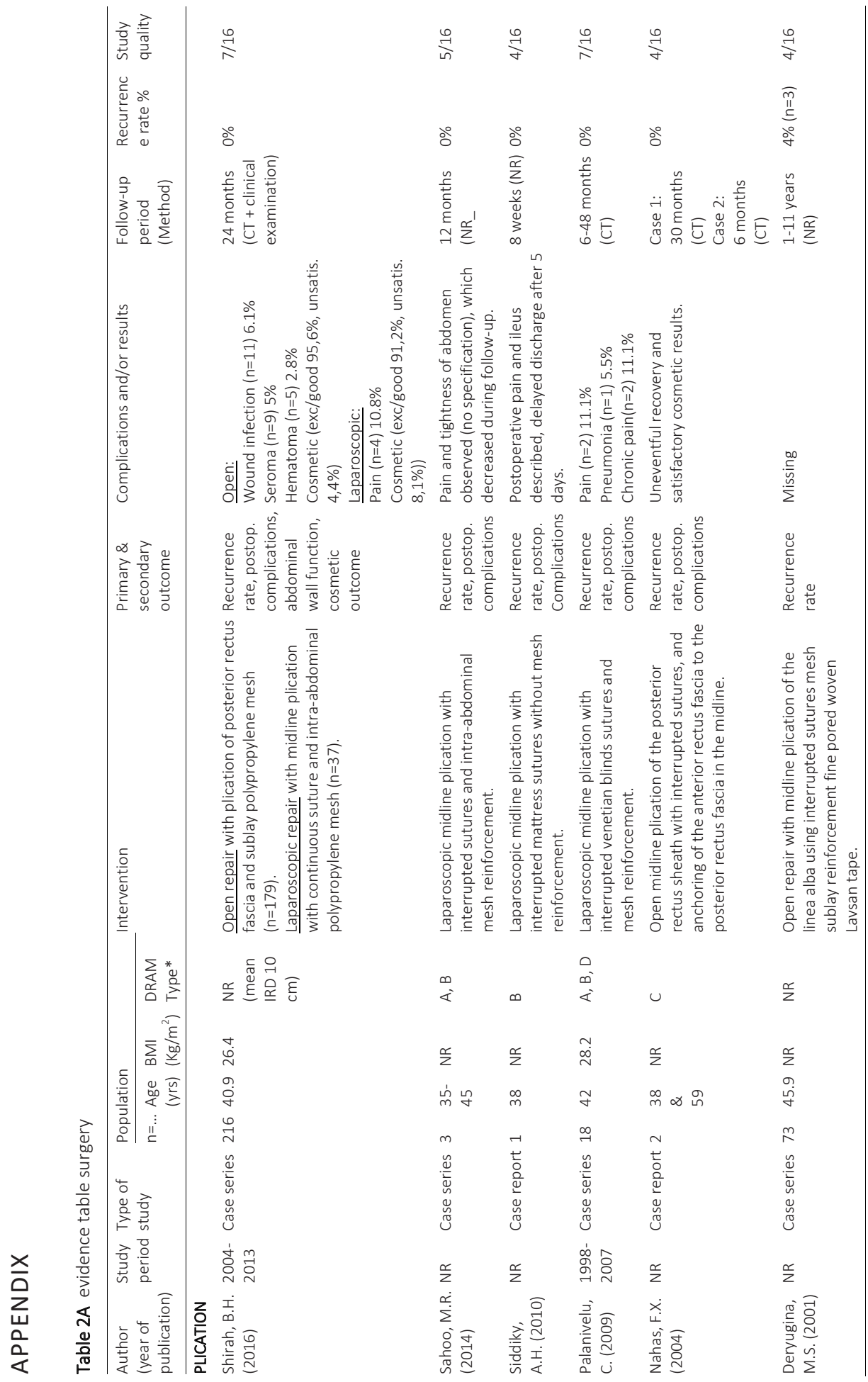


The general surgeon's perspective of rectus diastasis

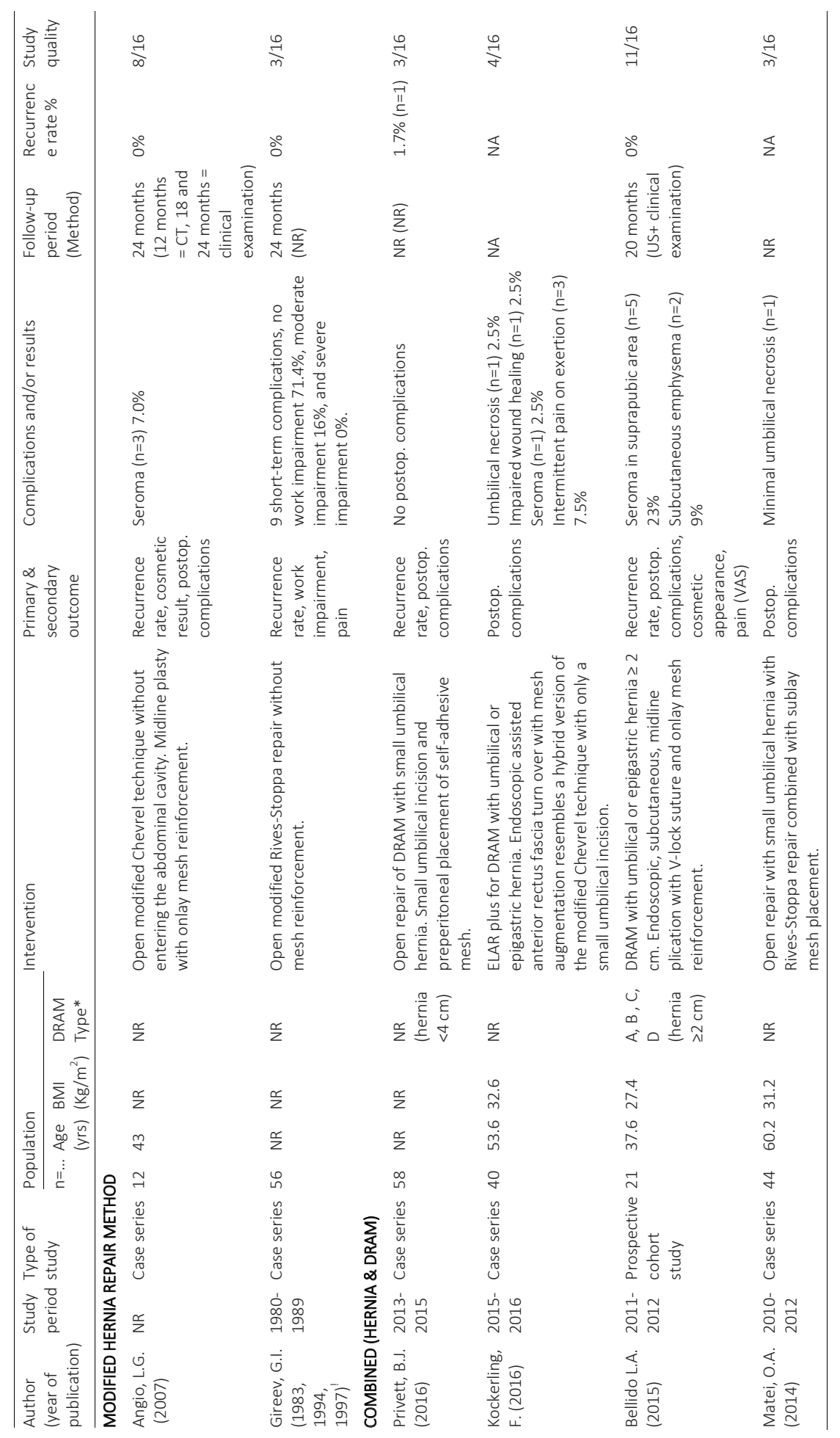




\section{Chapter 8}

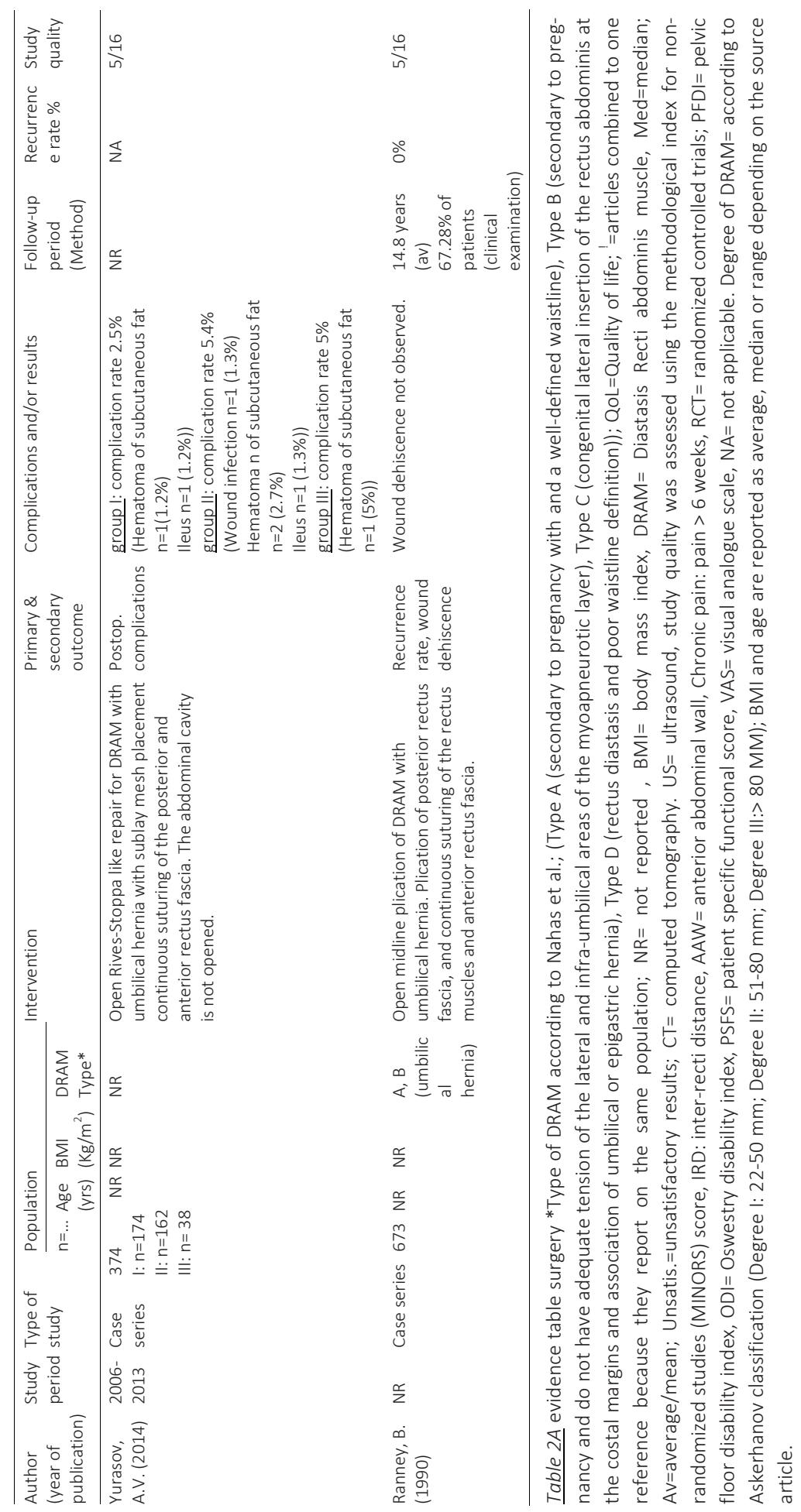




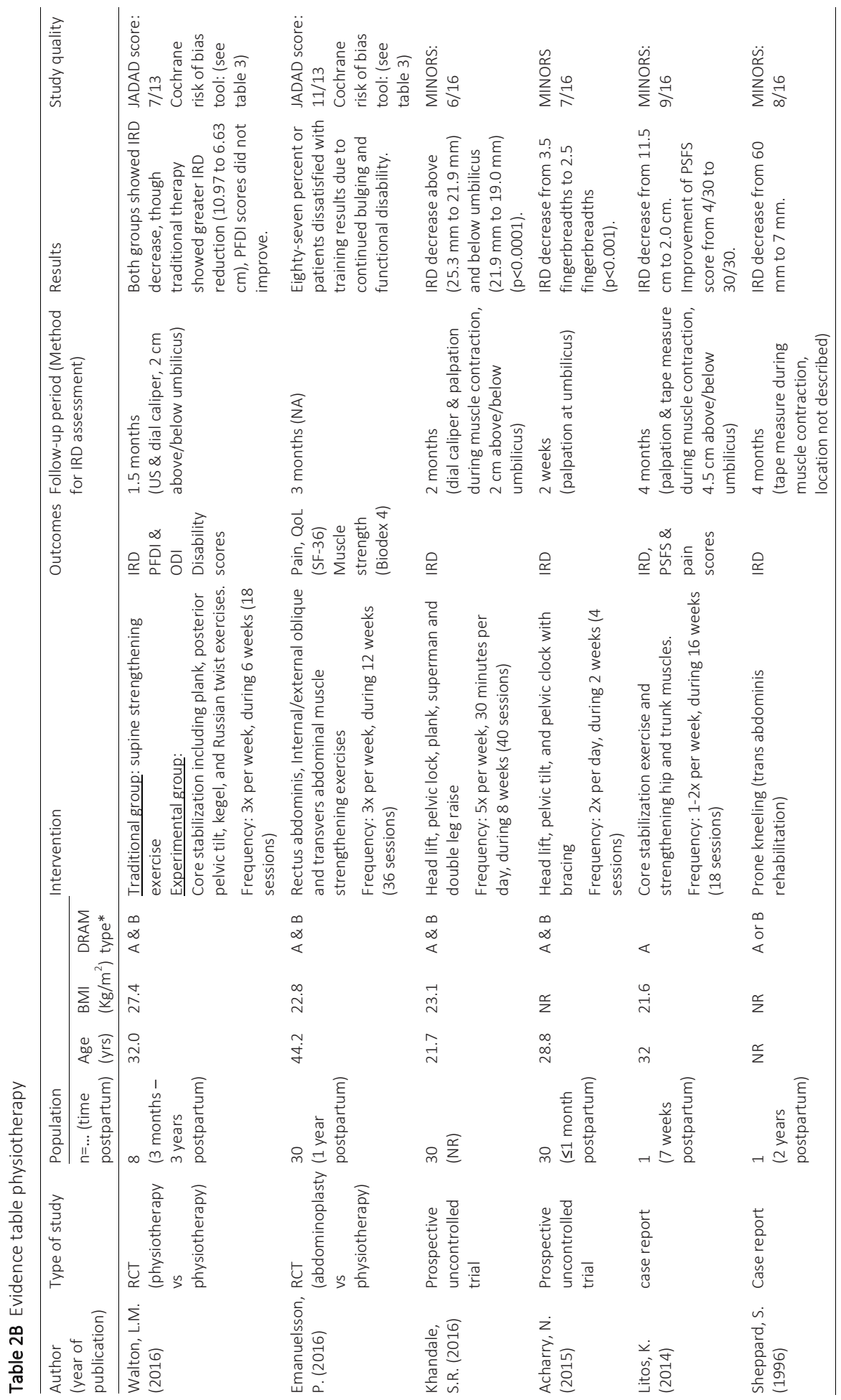


Chapter 8

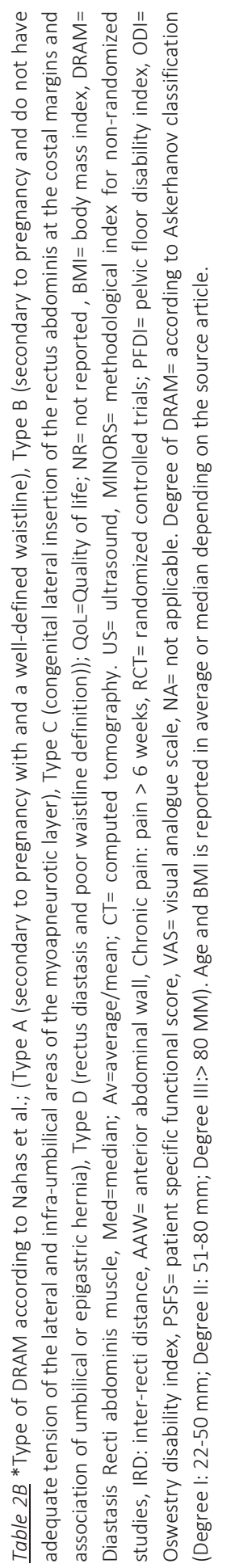




\section{PART}

\section{POSTOPERATIVE}





\section{Chapter}

\section{Patient-reported outcomes (PROs) after total extraperitoneal hernia repair (TEP)}

Elwin H.H. Mommers ${ }^{1,2}$, Dorien R.M. Hünen², Jolianne C.H.M. van Hout ${ }^{2}$, Mark Guijt ${ }^{2}$, Johannes A. Wegdam², Simon W. Nienhuijs ${ }^{3}$, Tammo S. de Vries Reilingh ${ }^{2}$

\footnotetext{
${ }^{1}$ Department of Surgery, Maastricht University Medical Center, Maastricht, The Netherlands ${ }^{2}$ Department of Surgery, Elkerliek hospital, Helmond, The Netherlands ${ }^{3}$ Department of Surgery, Catharina hospital, Eindhoven, The Netherlands
}

Hernia 2016 Dec;(21):45-50. doi:10.1007/s10029-016-1554-y. Epub 2016 Dec $5^{\text {th }}$ 


\section{ABSTRACT}

Background: Patient-reported outcomes (PROs) such as quality of life (QoL), patient satisfaction, and work impairment, are arguably the most important outcomes of any medical treatment. In 2011, Staerkle and Villiger developed the Core Outcome Measurements Index (COMI) to standardize PROs and PRO measurement for inguinal hernia patients, to increase inter-study comparability. The aim of this study is to prospectively evaluate the short- and long-term postoperative QoL, function, patient well-being, pain, and social/work disability, after total extraperitoneal (TEP) inguinal hernia repair and to provide the first clinical experience with the COMI-hernia questionnaire.

Methods: Between January 2013 and December 2014, all patients $\geq 18$ years that were scheduled for elective uni- or bilateral TEP in a regional hospital were approached to participate in this study. Measurements were taken preoperatively, and 6 weeks and 1 year postoperatively.

Results: One hundred and twenty patients (113 men, 7 women), mean age 59 years (SD \pm 12 ), completed the follow-up of 1 year. Ninety-seven percent of the population reported that the operation improved their complaints. QoL, function, well-being, and pain all improved after 6 weeks and 1 year after surgery. Patients experienced more social and work-related limitations 6 weeks after surgery compared to baseline measurements, though this improved to normal 1 year postoperatively. The incidence of chronic pain was 14\% (VAS C 2), which had a negative impact on the patients' sense of well-being.

Conclusion: Patients recovered well after TEP repair with a good quality of life and fast restore of function. Patient well-being was lower than expected due to a $14 \%$ incidence of chronic pain. The COMI-hernia scale provided reasonable insight into the patients' experience, though it was difficult to interpret for both patient and physician. 


\section{INTRODUCTION}

Inguinal hernia repair is one of the most frequently performed operations. ${ }^{1}$ In the Netherlands alone as much as 30.000 elective procedures are performed each year. ${ }^{2}$ Ever since the recurrence rates after inguinal hernia repair have dropped below 5\%, the focus of scientific research has slowly shifted away from recurrence rates, surgical techniques, and postoperative complications towards 'patient reported outcomes' (PROs). PROs are defined by the Food and Drug Administration (FDA) as 'a measurement based on a report that comes directly from the patient (i.e., study subject) about the status of a patient's health condition without amendment or interpretation of the patient's response by a clinician', ${ }^{3,4}$ Quality of life (QoL) is one of the most reported PROs and can be measured using generic QoL questionnaires, such as the SF-36(v2). ${ }^{5}$ These questionnaires can be used in a wide variety of populations, which is both their strength and weakness. They provide extensive multidimensional insight in the QoL, though lack the specificity to address key problems in specific populations. This lead to the development of Core Outcome Measurement Indexes (COMI's), which include a set of disease specific PROs that are important for a specific population. In 2011 Staerkle and Villiger developed a core outcome questionnaire specifically for inguinal hernia patients, the COMIHernia questionnaire. ${ }^{6}$ This validated questionnaire compares well to generic questionnaires such as the EQ-5D or EQ-VAS, and focuses on the patients perspective of specific problems for inguinal herniorrhaphy such as groin pain, testicular pain, pain during urination, work impairment, sensory disturbances, and patient satisfaction. ${ }^{6}$

Arguably, PROs are the most important outcomes of any medical treatment. Despite the importance of these data, a 2014 review of Antonescu states that between 2008 and 2012 only $16 \%$ of the 770 clinical articles published in the top 5 general surgery journals used at least one PRO. ${ }^{7}$ Only ten of these articles concerned hernia related research. Despite an increase in published PRO data during the last two years, to our knowledge, the COMI-hernia scale has never been used to evaluate the outcome of inguinal herniorrhaphy other than to original validation paper of Staerkle et al. ${ }^{6}$

This study is the first clinical prospective, single center analysis of PROs using the COMI-hernia scale. The aim of this study is to evaluate the short and long-term postoperative quality of life, function, patient well-being, pain, and social/work disability after total extraperitoneal inguinal hernia repair (TEP) and provide the first clinical experience of the COMI-hernia questionnaire.

\section{METHODS}

Between January 2013 and December 2014 all patients of at least 18 years old, that were scheduled for elective uni- or bilateral TEP herniorrhaphy in day-care surgery at a 
local hospital (Elkerliek Hospital, Helmond, The Netherlands), were approached for inclusion at the outpatient clinic.

All operations were performed under general anaesthesia by four experienced surgeons ( $\geq 300$ procedures/surgeon). TEP was performed using a conventional three port midline technique without the use of a balloon dissector. ${ }^{8}$ All patients received a preshaped anatomically curved polypropylene mesh (Bard Davol ${ }^{\circledR}$ inc., 3DMax Mesh ${ }^{\mathrm{TM}}$ ) that was not fixated with tackers or glue and therefore complies with the draft 2016 European hernia guidelines that states that mesh fixation in TEP is not necessary in almost all cases. ${ }^{9,10}$

Patients were asked to fill out the COMI-Hernia questionnaire during their preoperative consultation, the six-week postoperative consultation at the outpatient clinic, and after one year post-operative. The one-year postoperative questionnaires were sent via mail, patients were asked to return the questionnaire within three weeks. Patients who did not respond within this period received one reminder by telephone, in case the patient did not return the questionnaire after the reminder they were considered lost to follow-up for that measurement. All results were analysed by two authors simultaneous (EM, DH).

\section{COMI-hernia questionnaire}

The questionnaire consists of six pre-operative questions and twelve postoperative questions. The COMI-hernia questionnaire addresses five main outcomes; 'general QoL', 'function', 'patient well-being', 'pain', and 'social and work disability'. All outcomes, except social and work impairment and pain, are measured by a single question using a 5-point Likert scale. Social and work disability is measured by two questions and pain is measured on Visual Analogue Scale (VAS). A subscore can be calculated for each domain by converting the Likert scale in a 10-point scale as described in an article of Mannion et al. concerning the original COMI-spine questionnaire. ${ }^{11}$

QoL was evaluated using a single question: 'Please reflect on the last week. How would you rate your quality of life?'. Function was defined as the interference of the groin problem with normal work during the past week. Patient well-being was defined as the patient's willingness to live the rest of his/her life with the current groin problem. Clinically relevant pain was defined as a VAS $\geq 2$. Chronic pain was defined as a VAS $\geq 2$ one year postoperative according to the Medical Research Council Laparoscopic Groin Hernia Trial Group. ${ }^{12}$ Social and work disability was defined as the number of days' patients had to cut down from work or were kept from work during the past four weeks.

\section{Statistical analysis}

Statistical analysis was performed with SPSS version 22, SPSS Statistics for Windows, IBM corp. Armonk, NY, released 2013. Baseline characteristics were analysed using 
descriptive statistics. Comparison of ordinal outcome variables between the three measurements was performed using the Friedman test. In case of a statistically significant difference between the three groups $(\alpha=0.017)$ Wilcoxon signed rank tests were performed to identify the statistically significant $(\alpha=0.05)$ group. Continuous variables were compared using a one-way Anova test followed by post HOC analysis (Tukey HSD) $\alpha=0.05$. Comparison of core outcomes between uni- and bilateral inguinal hernia patients was performed using independent samples T-tests and Levene's test for equality of variances.

\section{RESULTS}

The flow of patients through the study is demonstrated in figure 1. One hundred and twenty patients (113 men, 7 women) with a median age of 61 years (range 32-86) and a complete follow-up period of one year were included in the analysis. Informed consent was obtained from all individual participants included in this study. Thirty-five pa-

Table 1 Demographic characteristics

\begin{tabular}{ll}
\hline Population size $n=120$ & \\
\hline Gender (male/female) $(n=)$ & $113 / 7$ \\
Age (median, range) & 61 years (32-86) \\
BMI (median, range) & $25 \mathrm{Kg} / \mathrm{m}^{2}(18-36)$ \\
Diabetes Type 2 ( $\mathrm{n}=)$ & 4 \\
& ASA I; $8(48 \%)$ \\
ASA classification ( $\mathrm{n}=)$ & ASA II; 56 (47\%) \\
& ASA III; 6 (5\%) \\
Operation time (median, range) & 41 min. (12-142) \\
Bilateral inguinal hernia repair $(n=)$ & $35(29 \%)$ \\
\hline
\end{tabular}

ASA= American Society of Anesthesiologists tients underwent bilateral inguinal hernia repair, the median operation time was 41 minutes (range 12-142) (table 1). Three patients had per-operative bleeding of an epigastric vessel that was successfully sutured. Eight patients had a postoperative complication; haematoma $(n=6)$, seroma $(n=1)$, or urinary tract infection $(n=1)$. Six patients (5\%) had a recurrence hernia within one year.

\section{Quality-of-life}

Six weeks after surgery $94 \%$ of the population reported either 'good or 'very good' QoL compared to $58 \%$ before the surgery. None of the patients scored their QoL as 'very bad'. One year after surgery $97 \%$ of the population reports 'good' or 'very good' QoL. There were no statistically significant differences between the QoL scores of patients with a haematoma or seroma versus the remainder of the population.

\section{Function}

Preoperative $65 \%$ of the population experienced 'a little bit' or 'moderate' function impairment and $10 \%$ reported 'a lot' or 'extreme' function impairment. Six weeks after surgery $66 \%$ reported no impairment and 33\% 'a little bit' or 'moderate' function im- 
pairment. One year after surgery $81 \%$ reports no impairment and $15 \%$ reports 'a little bit' of function impairment.

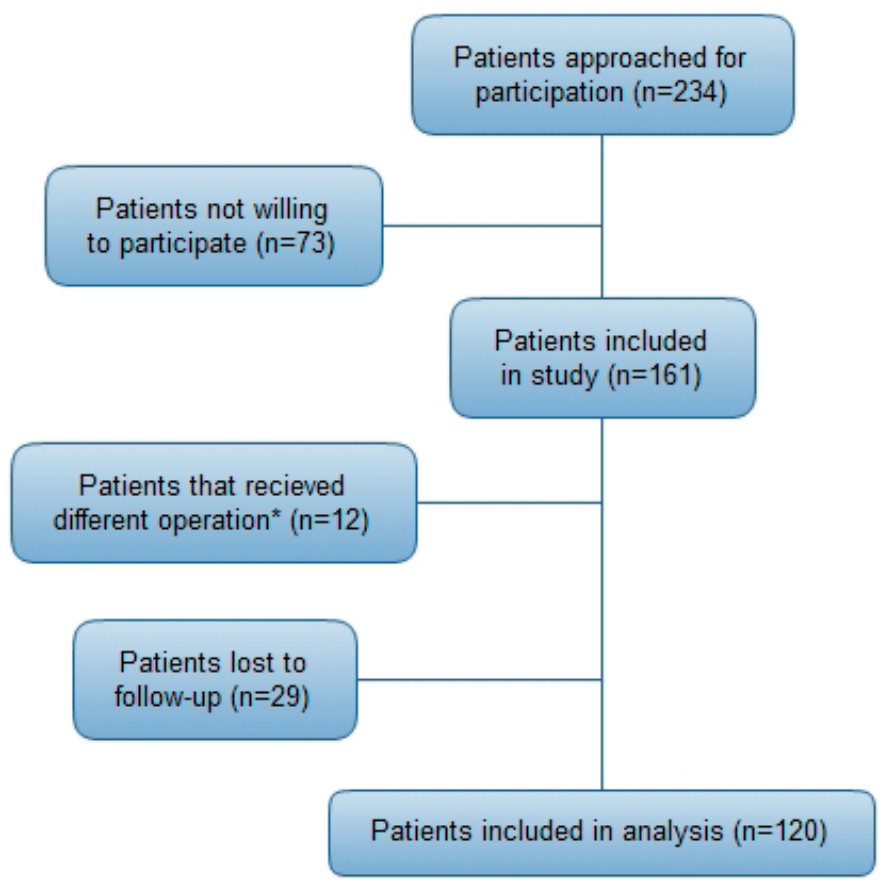

Figure 1 Flowchart of patients through study

Flowchart of patients through study. Flow of patient through study. ${ }^{*} 12$ patients were excluded after inclusion of the patient because the surgeon decided to perform a TAPP, Lichtenstein or Stoppa repair despite an initial indication for TEP.

\section{Patient well-being}

Preoperative patient well-being was low (64\% 'very dissatisfied'). Six weeks postoperative $76 \%$ of the population was satisfied with the result and $19 \%$ was either 'somewhat dissatisfied' or 'very dissatisfied'. The percentage of dissatisfied patients hardly improved over time as $15 \%$ of the patients remained somewhat or very dissatisfied one year after surgery. In terms of benefit from the operation $97 \%$ of the population reported that the operation 'helped' or 'helped a lot' six weeks postoperative. One year postoperative this percentage decreased to $93 \%$. At this time two patients reported that the operation made their complaints worse. During the preoperative period and first six weeks postoperative, all patients were satisfied with their 'overall medical care'. One year postoperative all but three patients were satisfied with their overall medical care. 
Pain

Seventy-one percent of the population (85 patients) reported pre-operative pain. Postoperative pain was reported by $19 \%$ of the population (22 patients) after six weeks and $14 \%$ (17 patients) after one year (chronic pain 14\% VAS $\geq 2 ; 10 \%$ VAS $\geq 3$ ). There was no statistically significant difference in the incidence of chronic pain amongst the four surgeons $(F(3,113)=0.384, p=0.765)$.

Roughly half of the patients that reported pain one year after surgery reported pain six weeks after surgery as well (8/17 patients). The remaining nine patients developed delayed onset chronic pain sometime between the six weeks and one-year measurement. One of these nine patients had a recurrent hernia.

The following specific forms of pain were reported six weeks after the operation: pain of the testicle $25 \%$, pain during urination $4 \%$, pain during intercourse $2 \%$. One year after surgery the prevalence of these complaints was: pain of the testicle $12.5 \%$, pain during urination $2.5 \%$, pain during intercourse $0 \%$.

\section{Social and work disability}

Sixty-one percent of the patients experienced interference in their daily activities or work to some extent. Six weeks after surgery $66 \%$ of patients reported limitations in their working ability, though the limitations they experienced were more severe compared to baseline (figure 2). Most limitations in work ability disappeared between the six weeks and one-year measurement as one year postoperative 90\% reported no limitation and $7 \%$ reported mild limitations during the past month.

Comparison of uni- and bilateral hernias

All preoperative main outcome scores were higher in the bilateral group compared to the unilateral group. These differences were not statistically significant. The one-year postoperative well-being score favoured the bilateral group (2.4 \pm 3.5 vs $1.0 \pm 2.5$ $p=0.010)$. The remaining postoperative outcomes were comparable between the two groups. 


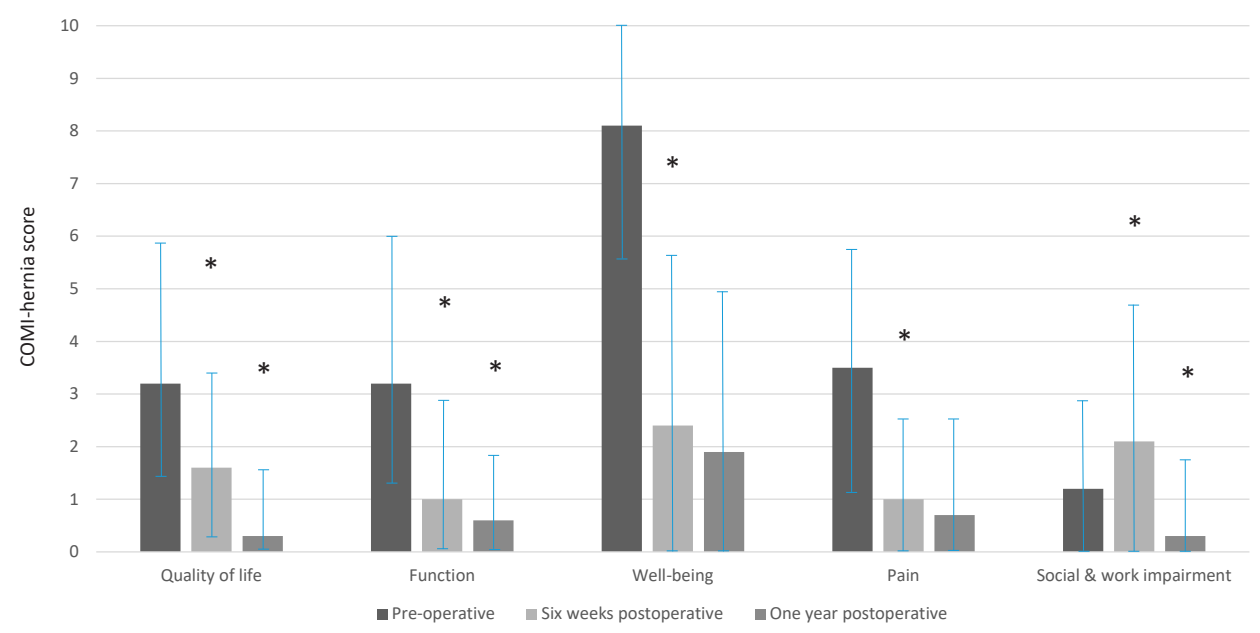

Figure 2 Main outcome parameters of the COMI-hernia questionnaire

Average main outcome scores of the COMI-hernia questionnaire reported on a 10-point scale. A higher score correlates with a worse outcome. *Score differs statistically significant from previous time of measurement; QoL preop.=3.2 (SD \pm 2.0$)$, QoL 6w postop.=1.6 (SD \pm 1.5$)$, QoL 1y postop.=0.3 (SD \pm 1.2$)$ (pre vs $6 \mathrm{w} p<0.001 ; 6 \mathrm{w}$ vs $1 \mathrm{y} p<0.001)$. Function preop.=3.2 $(\mathrm{SD} \pm 2.3)$, Function $6 \mathrm{w}$ postop. $=1.0(\mathrm{SD} \pm 1.6)$, Function $1 \mathrm{y}$ postop. $=0.6$ $(S D \pm 1.4)$ ) (pre vs $6 \mathrm{w} p<0.001 ; 6 \mathrm{w}$ vs $1 \mathrm{y} p=0.017)$. Well-being preop. $=8.1(\mathrm{SD} \pm 3.0)$, well-being $6 \mathrm{w}$ postop. $=2.4$ $(S D \pm 3.2)$, well-being $1 y$ postop. $=1.9(S D \pm 3.2)$ (pre vs $6 \mathrm{w} p<0.001 ; 6 \mathrm{w}$ vs $1 \mathrm{y} p=0.337$ ). Pain preop. VAS $=3.5$ $(S D \pm 2.7)$, pain $6 w$ postop. $=1.0(S D \pm 1.7)$, pain 1y postop. $=0.7(S D \pm 1.7)$ (pre vs $6 w p<0.001 ; 6 w$ vs $1 y p=0.017)$. So\&wo disability preop.=1.2 (SD \pm 1.9$)$, so\&wo 6w postop.=2.1 (SD \pm 2.6$)$, so\&wo 1y postop.=0.3 (SD \pm 1.0$)$ (pre vs $6 w p<0.001 ; 6 w$ vs $1 y p<0.001)$

\section{DISCUSSION}

This prospective study measured five core PROs in one-hundred and twenty inguinal hernia patients undergoing TEP herniorrhaphy and observed that most of the population recovered well within the first six postoperative weeks. However, six weeks from surgery patients still experienced clinically and statistically significantly more interference in their social life and work, compared to baseline scores. This did not seem to have a negative impact on their perceived QoL or well-being scores, which notably improved six weeks postoperative. Ujiki et al performed a similar prospective measurement of PROs after TEP repair and found that 'social functioning' and 'pain' did not improve immediately after surgery and may even worsen within the first three postoperative weeks. ${ }^{13}$ In our series, 'pain' notably improved six weeks after surgery. These results indicate that postoperative pain seems to resolve somewhere between the third and sixth postoperative week, though social function remains impaired for a longer period. Both in our series as in the series of Ujiki et al. social function scores returned to normal one year after surgery. We recommend future studies to explore the timeframe 
between six weeks and one year postoperative in more detail to identify the duration of postoperative social and work impairment.

Our series showed no clinically significant differences between patients with a unilateral and bilateral hernia despite a better well-being score in the bilateral group one year postoperative, which can be explained by the clear majority of patient with chronic pain having a unilateral hernia (82\%). Which is coherent with the previously published randomised clinical trial of Peeters et al. which compared lightweight and heavyweight meshes for inguinal hernia repair in term of quality of life. They did not observe any clinically or statistically significant differences between unilateral and bilateral hernia repairs in terms of QoL scores, return to work or pain perception, 1, 3, 6, and 12 months after surgery, regardless of the mesh used. ${ }^{14}$

In our series eight patients developed delayed onset ( $\geq 6$ weeks postoperative) chronic groin pain. A phenomenon that was previously observed in anterior repairs by Delikoukos et al. ${ }^{15}$ They report that the origin of the delayed onset pain can be found in mesh fixation and irritation of the ilioinguinal nerve, though this explanation does not translate well to endoscopic repairs, since the mesh is not fixated making mechanical stimulation of the ilioinguinal nerve less likely. ${ }^{16}$ Thermal irritation of the inguinal nerves has been suggested previously as possible cause for the delayed onset pain, though in our series no thermal cauterisation was used..$^{17}$ The delayed onset of the pain does suggest a neuropathic basis of the pain, therefore an inflammatory stimulation of the inguinal nerves or mechanical stimulation by 'kinking' of the inguinal nerves due to scar formation can serve as possible explanation.

The overall incidence of chronic pain was slightly higher than anticipated (14\% VAS $\geq 2 ; 10 \% V A S \geq 3$ ), and seemed to have a negative impact on the patient's sense of well-being. Though, this negative impact of chronic pain did not limit the patients in their perceived QoL, social or work function. Eighty-eight percent of the patients with chronic pain had preoperative pain, versus $67 \%$ of the remaining population $(p=0.147$ FEX), which could serve as an explanation for the relatively high incidence of chronic pain, since preoperative pain is a known risk factor for chronic pain. ${ }^{18,19}$

Previous studies, such as Bansal et al. reported a lower incidence of chronic pain (1.25\%, 3 months postoperative VAS $\geq 3$ ) after TEP repair. ${ }^{4}$ Other studies such as Ujiki et al. reported a chronic pain incidence $<2 \%$ based on the Carolina Comfort Scale outcome, though using the SF-36 13\% of the population reported more pain one year after surgery compared to baseline. ${ }^{13}$ Burgmans et al. included 473 patients to evaluate postoperative pain with the VAS score and Inguinal Pain Questionnaire. ${ }^{20}$ In their series, they found a chronic pain incidence of $10 \%$ defined as 'any pain' one year postoperative, as did Eklund et al. whom reported a similar incidence of $10 \%$ chronic pain one year postoperative in their prospective trial of 665 patients. ${ }^{21}$ A 2016 registry based study of Gutlic et al. reported a 7,7\% incidence of chronic pain using a cross-sectional analysis of 1110 patients. ${ }^{22}$ Overall, the incidence of chronic pain after inguinal hernia repair varies from $0-54 \%$ in published literature. ${ }^{17,23}$ The wide variety of chronic pain 
incidence can be explained by the overwhelming amount of data concerning the complex, multidimensional problem of postoperative pain, combined with inconsistent outcome definitions and measurement tools. ${ }^{24}$ This stipulates the need for standardised PROs, measured with standardised instruments to increase inter-study comparability.

The COMI-hernia questionnaire has attempted to standardise the most important PROs in inguinal hernia repair. The use of a single question to evaluate QoL worked well in clinical practice and seemed to provide an overall estimation of the self-perceived health status of the patient. However, the authors would argue that the COMI-hernia scale is difficult to interpret for patient and physician. The distinction between question five 'during the past four weeks, how many days did you cut down on the things you usually do because of your groin problem' and question six 'during the past four weeks, how many days did your groin problem keep you from going to work (job, school, housework)' confused the patients due to the similarity in outcome. Unemployed or retired patients frequently skipped question six because they felt it did not apply to them. Moreover, the subdomain 'function' defined as interference of the groin problem with normal work during the past week, closely resembles the subdomain of 'work and social impairment'. A suitable alternative to the COMI-hernia questionnaire could be the EuraHS-QoL questionnaire which was recently validated for inguinal hernia patients. ${ }^{25}$ This nine-item questionnaire is short, focuses on pain, function impairment and cosmetic result.

The present study has limitations. Only $75 \%$ of the included patients (120/161) completed the follow-up period of one year. The COMI-hernia questionnaire is a valid, reliable, and relatively short questionnaire though, is not a powerful tool in discriminating different origins of pain (i.e. during strenuous physical exercise, walking or lifting heavy objects). The original COMI-hernia questionnaire is validated in German, though published in English. For the present study, a Dutch translation was used. Despite these limitations, the authors feel that this prospective, single center series with experienced surgeons and clear description of mesh and fixation method provides enough evidence for the following conclusion.

\section{CONCLUSION}

The vast majority of patients recovered well after TEP repair with a good quality of life and fast restore of function. Patient well-being was lower than expected, possibly due to a relatively high incidence of chronic pain (VAS $\geq 2 ; 14 \%$ of the population). Chronic pain did not seem to effect long-term social and work disability. There were no clinically relevant differences between patients with a unilateral or bilateral hernia. The COMIhernia questionnaire provided reasonable insight in the patient's experience, although was difficult to interpret for both patient and physician. 


\section{ETHICAL APPROVAL}

All procedures performed in studies involving human participants were in accordance with the ethical standards of the institutional and/or national research committee and with the 1964 Helsinki declaration and its later amendments or comparable ethical standards.

\section{CONFLICT OF INTEREST DISCLOSURE}

SN reports grants from Johnson \& Johnson, and grants from Medtronic, outside the submitted work; All other authors declare that they have no conflict of interest. 


\section{REFERENCES}

1. Kingsnorth A, LeBlanc K. Hernias: inguinal and incisional. The Lancet 2003;362:1561-71.

2. Simons MP, de Lange D, Beets GL, van Geldere D, Heij HA, Go PM. [The 'Inguinal Hernia' guideline of the Association of Surgeons of the Netherlands]. Nederlands tijdschrift voor geneeskunde 2003;147:2111-7.

3. FDA. US Department of Health and Human Services Food and Drug Administration Center for Drug Evaluation and Research (CDER); Center for Biologics Evaluation and Research (CBER); Center for Devices and Radiological Health (CDRH); Guidance for Industry Patient-Reported Outcome Measures: Use in Medical Product Development to Support Labeling Claims 2009:1-43.

4. Bansal VK, Misra MC, Babu D, et al. A prospective, randomized comparison of long-term outcomes: chronic groin pain and quality of life following totally extraperitoneal (TEP) and transabdominal preperitoneal (TAPP) laparoscopic inguinal hernia repair. Surgical endoscopy 2013;27:2373-82.

5. Ware JE, Jr. SF-36 health survey update. Spine 2000;25:3130-9.

6. Staerkle RF, Villiger P. Simple questionnaire for assessing core outcomes in inguinal hernia repair. The British journal of surgery 2011;98:148-55

7. Antonescu I, Mueller CL, Fried GM, Vassiliou MC, Mayo NE, Feldman LS. Outcomes reported in highimpact surgical journals. Br J Surg 2014;101:582-9. doi: 10.1002/bjs.9450. Epub 2014 Feb 26.

8. Lange JF, Rooijens PPGM, Koppert S, Kleinrensink GJ. The preperitoneal tissue dilemma in totally extraperitoneal (TEP) laparoscopic hernia repair. Surgical Endoscopy And Other Interventional Techniques 2002;16:927-30.

9. Bell RC, Price JG. Laparoscopic inguinal hernia repair using an anatomically contoured three-dimensional mesh. Surgical endoscopy 2003;17:1784-8.

10. HerniaSurge. HerniaSurge Guidelines, Statements and Recommendations. http://herniasurgecom/ contact/ 2016.

11. Mannion AF, Elfering A, Staerkle R, et al. Outcome assessment in low back pain: how low can you go? European Spine Journal 2005;14:1014-26.

12. Grant AM, Scott NW, O'Dwyer PJ. Five-year follow-up of a randomized trial to assess pain and numbness after laparoscopic or open repair of groin hernia. The British journal of surgery 2004;91:1570-4.

13. Ujiki MB, Gitelis ME, Carbray J, et al. Patient-centered outcomes following laparoscopic inguinal hernia repair. Surgical endoscopy 2015;29:2512-9.

14. Peeters $E$, Spiessens $C$, Oyen $R$, et al. Laparoscopic inguinal hernia repair in men with lightweight meshes may significantly impair sperm motility: a randomized controlled trial. Ann Surg 2010;252:240-6.

15. Delikoukos S, Fafoulakis F, Christodoulidis G, Theodoropoulos T, Hatzitheofilou C. Re-operation due to severe late-onset persisting groin pain following anterior inguinal hernia repair with mesh. Hernia : the journal of hernias and abdominal wall surgery 2008;12:593-5.

16. Reinpold W, Schroeder AD, Schroeder M, Berger C, Rohr M, Wehrenberg U. Retroperitoneal anatomy of the iliohypogastric, ilioinguinal, genitofemoral, and lateral femoral cutaneous nerve: consequences for prevention and treatment of chronic inguinodynia. Hernia : the journal of hernias and abdominal wall surgery 2015;19:539-48.

17. Poobalan AS, Bruce J, Smith WC, King PM, Krukowski ZH, Chambers WA. A review of chronic pain after inguinal herniorrhaphy. The Clinical journal of pain 2003;19:48-54.

18. Poobalan AS, Bruce J, King PM, Chambers WA, Krukowski ZH, Smith WC. Chronic pain and quality of life following open inguinal hernia repair. The British journal of surgery 2001;88:1122-6.

19. Dennis R, O'Riordan D. Risk Factors for Chronic Pain After Inguinal Hernia Repair. Annals of The Royal College of Surgeons of England 2007;89:218-20.

20. Burgmans JP, Schouten N, Clevers GJ, et al. Pain after totally extraperitoneal (TEP) hernia repair might fade out within a year. Hernia : the journal of hernias and abdominal wall surgery 2015;19:579-85.

21. Eklund A, Montgomery A, Bergkvist L, Rudberg C. Chronic pain 5 years after randomized comparison of laparoscopic and Lichtenstein inguinal hernia repair. The British journal of surgery 2010;97:600-8. 
22. Gutlic N, Rogmark P, Nordin P, Petersson U, Montgomery A. Impact of Mesh Fixation on Chronic Pain in Total Extraperitoneal Inguinal Hernia Repair (TEP): A Nationwide Register-based Study. Ann Surg 2016;263:1199-206.

23. Nienhuijs S, Staal E, Strobbe L, Rosman C, Groenewoud H, Bleichrodt R. Chronic pain after mesh repair of inguinal hernia: a systematic review. American journal of surgery 2007;194:394-400.

24. Bhangu A, Singh P, Pinkney $T$, Blazeby JM. A detailed analysis of outcome reporting from randomised controlled trials and meta-analyses of inguinal hernia repair. Hernia : the journal of hernias and abdominal wall surgery 2015;19:65-75.

25. Muysoms FE, Vanlander A, Ceulemans R, et al. A prospective, multicenter, observational study on quality of life after laparoscopic inguinal hernia repair with ProGrip laparoscopic, self-fixating mesh according to the European Registry for Abdominal Wall Hernias Quality of Life Instrument. Surgery 2016. 



\section{0}

\section{EHS adhesion score: an international}

consensus-based mesh-tissue adhesion score

Leontine C.L. van den Hil ${ }^{1}$, Elwin H.H. Mommers ${ }^{1}$, Anne-Claire J.W.A.M. Bosmans ${ }^{1}$, Salvador Morales-Conde ${ }^{2}$, Verónica Gomez-Gil ${ }^{3}$, Karl A. Leblanc ${ }^{4}$, Aude Vanlander ${ }^{5}$, Emmelie Reynvoet ${ }^{5}$, Frederik Berrevoet ${ }^{5}$, Simone Gruber-Blum ${ }^{6}$, Ediz Altinli ${ }^{7}$, Corey R. Deek$\mathrm{en}^{8}$, René H. Fortelny, Jan Willem Greve ${ }^{10}$, Koen Chiers ${ }^{11}$, Ruth Kaufmann ${ }^{12}$, Johan F. Lange $^{12}$, Uwe Klinge ${ }^{13}$, Marc Miserez ${ }^{14}$, Alexander H. Petter-Puchner ${ }^{6,9}$, Nicole D. Bouvy ${ }^{1}$, Marc H.F. Schreinemacher ${ }^{15}$

${ }^{1}$ Department of Surgery, Maastricht University Medical Center, Maastricht, The Netherlands

${ }^{2}$ Unit of Innovation and Minimally Invasive Surgery, Hospital Universitary Virgen Del Rocío, Seville, Spain ${ }^{3}$ Department of Medicine and Medical Specialties, Networking Research Center on Bioengineering, Biomaterials and Nanomedicine (CIBER-BBN), University of Alcalá, Alcalá de Henares, Madrid, Spain

${ }^{4}$ Our Lady of the Lake Physician Group, Minimally Invasive Surgery Institute, Baton Rouge, Louisiana, United States of America

${ }^{5}$ Department of General and Hepatobiliary Surgery, Ghent University Hospital, Ghent, Belgium ${ }^{6}$ Ludwig Boltzmann Institute for Experimental and Clinical Traumatology, Austrian Cluster for Tissue Regeneration, Vienna, Austria ${ }^{7}$ Department of General Surgery, Bilim University, Istanbul, Turkey ${ }^{8}$ Covalent Bio LLC, Saint Louis, Missouri, United States of America ${ }^{9}$ Department of General Surgery, Wilhelminenspital der Stadt Wien, Vienna, Austria ${ }^{10}$ Department of General Surgery, Zuyderland Medical Center, Heerlen, The Netherlands ${ }^{11}$ Department of Pathology, Bacteriology and Poultry Diseases, Faculty of Veterinary Medicine, Ghent University, Ghent, Belgium

${ }^{12}$ Department of Surgery, Erasmus Medical Center Rotterdam, Rotterdam, The Netherlands

${ }^{13}$ Department of General, Visceral and Transplantation Surgery, RWTH Aachen University Hospital, Germany

${ }^{14}$ Department of Abdominal Surgery, University Hospitals, KU Leuven, Leuven, Belgium

${ }^{15}$ Department of General Surgery, Academic Medical Center, Amsterdam, The Netherlands

Work in progress 


\section{ABSTRACT}

Background: The use of surgical mesh to reinforce the abdominal wall has become common practice in modern surgery to prevent or repair abdominal wall hernias. Unfortunately, the use of surgical mesh inside the abdominal cavity frequently result in adhesion formation to the mesh. Currently there is no consensus on the clinical scoring of postoperative adhesion in the presence of a mesh. Hence, interstudy comparability is impaired. The aim of this study is to develop an internationally recognised clinical adhesion score using a modified DELPHI-method (RAND-UCLA).

Methods: A panel of experts was selected based on previously published adhesions scores and screening of high-impact publications on postoperative adhesion scoring. All experts were asked their opinion regarding four items; 1 ) general questions regarding the usefulness of adhesion scoring models in both experimental and clinical setting, 2) preferred design of the adhesion score, 3) appropriateness of macroscopic variables, and 4) appropriateness of microscopically scoring models. This study comprised of two questionnaires-based round and one international consensus meeting during the European Hernia Congress 2016 in Rotterdam.

Results: The first questionnaire was completed by $82 \%(n=23)$ of the panel members. From the 23 participants that completed the first questionnaire, 18 completed the follow-up questionnaire, and ten participated in the final meeting. A total of 140 different items were included in this study. Consensus was reached on $56 \%$ of the presented items.

Conclusion: The EHS 2016 consensus score presented in this paper can be used for mesh-related adhesions and is based on the opinion of 18 international leading experts in the field of mesh-based adhesions. We recommend the use of this score in future experimental and clinical research to increase the inter-study comparability. Adhesions should be measured objectively and the highest score per variable should be used in the scoring system. 


\section{INTRODUCTION}

Incisional hernias are a frequent problem after laparotomy, requiring mesh repair in approximately $25 \%$ of all patients. ${ }^{1}$ A major concern regarding intra-abdominal mesh placement is the formation of adhesions. Radiological signs of adhesions are present in $67-100 \%$ of all patients that underwent abdominal surgery with or without mesh. ${ }^{2}$ Approximately $5-18 \%$ of these patients will develop complications due to these adhesions. $^{3-5}$ Intra-abdominal mesh placement is a known inducer of adhesions between bowel content and mesh. ${ }^{6}$ Intra-abdominal adhesions are known cause for serious postoperative complications such as bowel obstruction. ${ }^{7,8}$ To prevent such complications, meshes for intra-abdominal use are often coated with anti-adhesive materials, and anti-adhesive devices can be applied inside the abdominal cavity to prevent adhesions. Despite the abundance of anti-adhesive devices, positive clinical results are scares. $^{9,10}$

Important factors influencing adhesion formation are mesh material, pore size, and the presence of antiadhesive coatings on the visceral side of the mesh. These factors reduce the number of adhesions, though complete adhesion prevention is currently not possible. ${ }^{11,12}$ Before clinical application of anti-adhesive devices, they are often tested in animal models. ${ }^{13,14}$ Comparing the efficacy of anti-adhesive devices has become increasingly difficult due to the abundance of adhesion scores. ${ }^{15,16}$ There are currently over twenty different adhesion scores available. Diamond et al. described one of the first scoring systems, which used sub scores for extent, type, and tenacity of adhesions. ${ }^{15}$ There is currently no consensus on the ideal adhesion scoring method, and inter-study comparability is decreasing due to the increasing diversity in adhesion scores. ${ }^{15-18}$

The goal of this study is to use the DELPHI-method to reach international consensus on an adhesion scoring method focussing on mesh-related adhesions amongst an international panel of experts to increase consistent outcome reporting in the field of intraabdominal mesh-related adhesions. ${ }^{19}$

\section{METHODS AND MATERIALS}

The DELPHI method is an accepted technique for evaluation and reach consensus on a topic. In short in comprises of multiple rounds of questionnaires, which are filled out by an expert panel. The DELPHI process is characterised by anonymity, controlled feedback to the expert panel, and statistical analysis of the results. ${ }^{20}$ Anonymity reduces the influence of dominant individuals in the panel, and it is thought that experts might change their opinion after providing input of the panel. This study used a modified DELPHI method named RAND-UCLA Appropriateness Method (RAM), which is a DELPHI-scores 
specifically aimed at the medical field. ${ }^{21}$ The RAM starts with performing a systematic review, followed by questionnaires, and eventually a face-to-face meeting.

\section{Panel formation}

The expert panel was formed based on the results of a systematic literature search on adhesion scoring models. Any author whom had at least three publications on adhesion scoring systems or (mesh) adhesion related complications was invited to participate in the expert panel. After the first invitation, two personalized reminders were sent after one and two weeks. In case the author did not respond to the reminders, they were considered unreachable and were not included in the expert panel.

\section{Questionnaires}

Questionnaires were designed and distributed online (SurveyMonkey Inc, Palo Alto, CA). The questionnaires addressed multiple choice questions regarding four main items; 1 ) general questions regarding the usefulness of adhesion scoring models in both experimental and clinical setting, 2) preferred design of the adhesion score, 3) appropriateness of macroscopic variables, and 4) appropriateness of microscopically scoring models. Panel members could rate these questions on a nine-point Likert scale, ranging from very inappropriate (score 1) to very appropriate (score 9). Participants were encouraged to provide additional remarks in a free text fields, such as other important variables or feedback on their answers.

At the end of each round the results were statistically analysed and presented in during the follow-up round. Participant received a copy of their individual answers separately. Any item on which consensus was reached was not included in the follow-up rounds. Items without consensus were repeated and adjusted if required, according to comments received in the previous round.

\section{Final consensus meeting}

The last round of the DELPHI analysis was a face-to-face meeting held during the $38^{\text {th }}$ (2016) International Congress of the European Hernia Society (EHS) in Rotterdam, The Netherlands. During this meeting, any remaining items without consensus were discussed. Any panel member who finished two questionnaire rounds was invited to participate in the meeting.

Each item was presented with a short introduction and scored on a nine-point Likert scale by each individual participant. A summary of the discussion was sent to the entire panel, followed by two suggestions for a new adhesion scoring model. Panel members were asked which model had their preference. 
Statistical analysis

All data was analysed using SPSS statistics for Windows, IBM corp. Armonk, NY, released 2013. Consensus was reached if the panel rated a topic unanimously as either inappropriate (panel median 1-3) or appropriate (panel median 7-9), without disagreement. Disagreement was tested using the inter-percentile range adjusted for symmetry, according to the RAND-UCLA Appropriateness Method Manual and as used by Moossdorff et al. $^{21,22}$

\section{RESULTS}

Invitations for participation in the 2016 mesh-related adhesions DELPHI were sent to 57 authors. Thirty-seven authors responded, of which nine declined the invitation and 28 accepted (49\% acceptance rate). One author declined the invitation though sent another experienced researcher in the field of adhesions to take his place. The first questionnaire was completed by $82 \%(n=23)$ of the panel members. From the 23 participants that completed the first questionnaire, 18 (64\%, surgeon $(n=12)$, postdoc. Researcher $(n=3)$, professor $(n=9))$ completed the follow-up questionnaire, and ten (36\%) participated in the final meeting.

\section{Panel characteristics}

All panel members are affiliated to a university and are experienced in performing both animal and clinical research in the field of intra-abdominal mesh-related adhesions. The panel members participated in the DELPHI analyses on their own behalf and expertise, no compensation of any kind was provided to the participants. Any personal views of the individual participants do not necessarily reflect the view of their affiliated institutions.

\section{First Questionnaire}

The first questionnaire consisted of 64 items in seven categories, 1) utility of scoring models, 2) adhesion related complications, 3) macroscopic outcome, 4) scoring scales, 5) composite versus subscores, 6) histological assessment, and 7) follow-up. After the first round, consensus was reached on 44/64 items (69\%) of the presented items. Disagreement existed on the remaining 20 items. Fourteen of these 20 items were rephrased into 22 separate items. Combined with the six unchanged items, 28 remaining items were included in the second questionnaire. See figure 1 for the flow of information throughout the study. 


\section{Second Questionnaire}

The second questionnaire consisted of 76 items (28 from the first questionnaire, 48 new items for adhesion model development). Most questions that were added addressed the simplification of a new adhesion scoring model that would be discussed during the final meeting.

At the end of the second round, consensus was reached on 34/76 items (45\%) of the presented items. Consensus was achieved on 1/6 of the unchanged items, 8/22 of the adjusted items and $25 / 48$ of the new items. Including both questionnaire round, consensus was reached on 78/140 items (56\%).

\section{Final meeting}

The goal of the meeting was to formulate the final variables that should be included in a new adhesion scoring model. Furthermore, the most appropriate scoring scales and follow-up times had to be selected. Feedback on the first two rounds was provided to the participants before the final meeting. During the meeting, items on which no consensus was reached (62 questions, regarding 18 topics) were discussed.

\section{Consensus based Adhesion Scoring Model}

All panel members consented that mesh-tissue adhesions should be systematically scored in both human and experimental settings, and that a uniform consensus-based scoring method should be used to increase inter-study comparability. According to the panel members, mesh-related adhesion scores should exist primarily of macroscopic variables. Microscopic variables can also be assessed, though should be presented separately from the macroscopic score. Items that should be included in the macroscopic adhesion score are; percentage of mesh surface covered with adhesions, strength needed to divide adhesions (tenacity), thickness of adhesions, and organ involvement. The absolute number of adhesions and the macroscopic vascularization are considered inappropriate items for adhesion scoring.

Any new scoring system for intra-abdominal mesh-tissue adhesions should be based on a 4- or 5-point scale to allow adequate variation in responses and statistical analyses.

Finally, follow-up times in experimental research should be a minimum of four weeks after mesh-implantation. Follow-up of clinical studies on mesh related adhesions should include a minimal follow-up of 6 months. A new consensus-based adhesion score including the abovementioned recommendations, the 2016 EHS consensus score, is presented in table 1. 


\begin{tabular}{|ll|}
\hline Results of the RAND-UCLA (DELPHI) 'mesh related adhesion' consensus analysis \\
- Utility of scoring models & - Composite versus subscores \\
- Adhesion related complications & - Histological assessment \\
- Macroscopic outcome & - Follow-up \\
- Scoring scales & \\
\hline
\end{tabular}

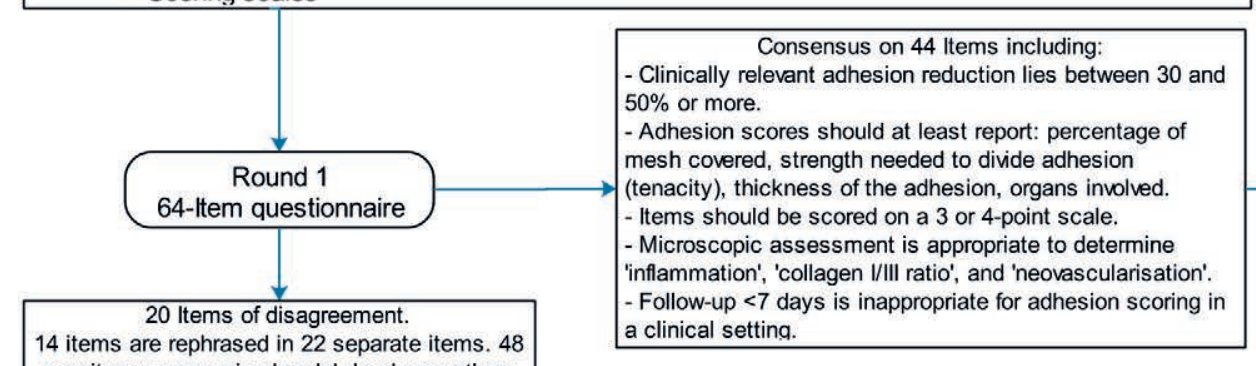
new items concerning 'model development' are added, 6 Items are are entered unchanged

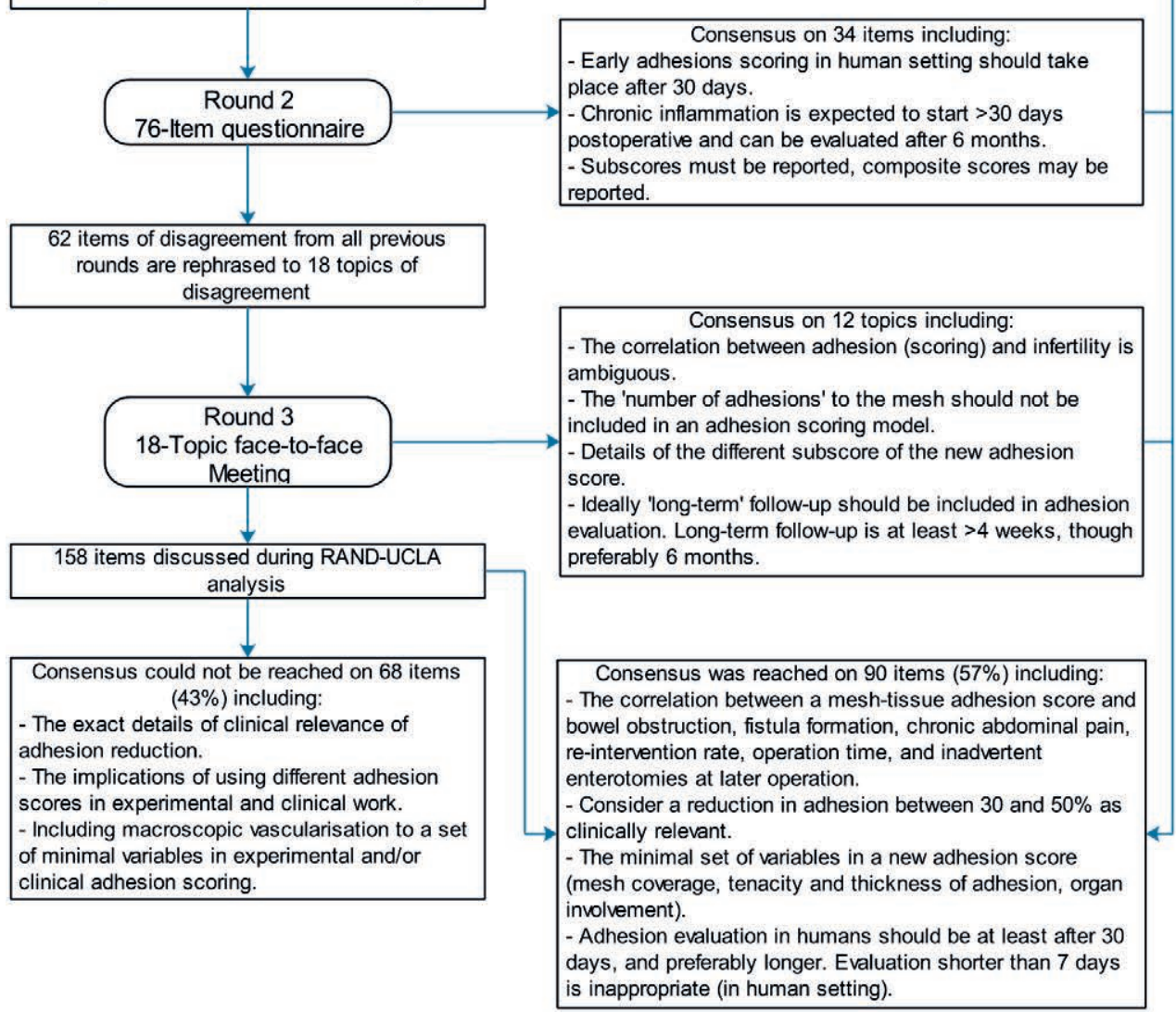

Figure 1 Flow of information through the DELPHI analysis

The DELPHI analysis consisted of three rounds and resulted in a consensus on 57\% of the topics discussed. The figure provides a flowchart of information throughout the DELPHI analysis, combined with the most important consensus reached in each round. 
Table 1 EHS 2016 adhesion consensus score

\begin{tabular}{ll}
\hline Item & Score \\
\hline No adhesions & 0 \\
Percentage of mesh surface covered with adhesions & 1 \\
$1-25 \%$ & 2 \\
$26-50 \%$ & 3 \\
$51-75 \%$ & 4 \\
$76-100 \%$ & 1 \\
Tenacity & 2 \\
Loose adhesions easily released bluntly & 3 \\
Adhesions require sharp dissection, no organ/serosal damage & 1 \\
Adhesions require sharp dissection, with unavoidable organ/serosal damage & 2 \\
Thickness of adhesions & 2 \\
Single thin, filmy adhesion & 3 \\
Multiple thin, filmy adhesions & 3 \\
Single dense adhesion with or without filmy adhesions & 4 \\
Multiple dense adhesions with or without filmy adhesions &
\end{tabular}

The EHS 2016 adhesion consensus score consist of four main items. 1) 'Percentage of mesh surface covered with adhesion' can either be scored by automated computer analysis of pictures taken during surgery, or be estimated by the surgeon. 2) 'Tenacity' depends on the tools needed for adhesiolysis and relates to the strength of the adhesion. 3) 'Thickness of adhesions' is divided in four categories of adhesions. Macroscopically two types of adhesions can be scored (flimsy or dense) the categories are based on either singular or multiple adhesions of both types. 4) 'Organ involvement' scores the adhered structures and increases in severity with increased adherence to the intestinal tract. The most severe category describes adhesions to the intestinal tract combined with macroscopic damage or interference with the organ such as fistula formation or bowel erosion. When reporting the EHS 2016 adhesion consensus score each individual score must be reported to increase comparability between publications. An overall score can be calculated by adding all individual categories, though is considered less relevant.

\section{Discussion}

Due to the abundance of adhesion scores and lack of consensus in relevant outcomes measures, comparability in mesh related adhesion research is low. This DELPHI analysis consulted eighteen leading experts in the field of adhesion research to create a minimum set of outcome variable with international consensus. Based on this set of variables, a new adhesion score, the EHS 2016 adhesion consensus score was developed. ${ }^{21}$ Consensus was reached on $57 \%$ of the discussed items. The moderate level of consensus is to be expected in a field that is still developing. Despite the remaining disagreement on several items, this study succeeded in defining a set of minimal outcome pa- 
rameters and gives consensus-based recommendations for follow-up and clinical efficacy in mesh related adhesion research such as the recommended follow-up for experimental animal studies of at least four weeks, and minimal follow-up for clinical studies of six months.

The EHS adhesion consensus score is based on four equally important macroscopic variables; mesh coverage, tenacity, thickness, and organ involvement. The EHS 2016 adhesion consensus score can be interpreted as a minimal set of outcome criteria, hence it can be used as scoring system, or as part of larger set of outcome variables. All variables of the EHS 2016 adhesion consensus score should be scored separately. A total score can be computed, though to compare results between studies accurately, each subscore must be reported separately.

To maximize inter-study comparability, it is important to use the same score in a similar manner and with comparable follow-up times. Recommended follow-up times are at least four weeks in experimental animal studies and six months in clinical studies. To assess objectively the percentage of the mesh covered with adhesions standardized pictures can be taken and analysed using a computer program. Another form of objective adhesion measurement consists of using a grid of four quadrants to compute the percentage of mesh coverage.

Subdivisions of tenacity are objectively measurable, although it must be mentioned that it is only appropriate to score adhesions as loose and easily to release bluntly (score 1 ) if all tissue is removed from the mesh. If tissue remains on the mesh, adhesions require sharp dissection and should thus be scored as score 2 of this variable.

The EHS 2016 adhesion consensus score was developed by leading authorities in the field of adhesion research. Although not all invited researchers responded to our invitation to participate in this consensus study. Authors who did reply and became panel member are interested in the topic and are convinced that a uniform scoring method is needed. This process may have led to selection bias, though the restricted number of panel members is peculiar to a consensus project. The authors consider the current panel to be representative for all researchers who perform research on mesh related adhesions.

Unfortunately, none of the previously published adhesion scores has been clinically validated, nor het the EHS 2016 adhesion consensus score. It is still unknown whether the variables included in the EHS 2016 adhesion consensus score are related to clinical outcomes of adhesive disease as the current level of evidence is expert opinion. Although it seems likely that a comprehensive presence of dense adhesions requiring sharp dissection leads to more complications than limited presence of filmy adhesions, only a relation between small bowel adhesions leading to obstruction has previously been described. ${ }^{8}$ Furthermore, it is shown that a higher adhesion score is associated with an increased risk of enterotomies. ${ }^{23}$ Ideally, functional impairment and organ injuries due to adhesions or adhesiolysis are added to a mesh-related adhesion score, since these are the most important outcomes that should be prevented. However, functional 
impairment is difficult to assess in experimental animal research. Therefore, adhesions between mesh and intestines are used as proxy for functional impairment.

\section{Conclusion}

The EHS 2016 adhesion consensus score is the result of a DELPHI analysis amongst an international panel of adhesion experts. The score defines a minimal set of outcome parameters and should be measured as objective as possible. A total score can be computed, though it is inferior to the presentation of all individual sub scores. To increase comparability in mesh related adhesion research, the variables of the EHS 2016 adhesion consensus score should be the minimal set of outcomes reported. 


\section{REFERENCES}

1. Burger JW, Luijendijk RW, Hop WC, Halm JA, Verdaasdonk EG, Jeekel J. Long-term follow-up of a randomized controlled trial of suture versus mesh repair of incisional hernia. Ann Surg 2004;240:578-83; discussion 83-5.

2. Ouaïssi M, Gaujoux S, Veyrie N, et al. Post-operative adhesions after digestive surgery: Their incidence and prevention: Review of the literature. Journal of Visceral Surgery 2012;149:e104-e14.

3. Ellis H, Moran BJ, Thompson JN, et al. Adhesion-related hospital readmissions after abdominal and pelvic surgery: a retrospective cohort study. Lancet 1999;353:1476-80.

4. Hellebrekers BW, Kooistra T. Pathogenesis of postoperative adhesion formation. Br J Surg 2011;98:150316. doi: 10.002/bjs.7657. Epub 2011 Aug 23.

5. Wilson MS. Practicalities and costs of adhesions. Colorectal Dis 2007;9:60-5.

6. Luijendijk RW, de Lange DC, Wauters CC, et al. Foreign material in postoperative adhesions. Annals of Surgery 1996;223:242-8.

7. Turza KC, Butler CE. Adhesions and meshes: synthetic versus bioprosthetic. Plastic and reconstructive surgery 2012;130:206s-13s.

8. ten Broek RP, Issa Y, van Santbrink EJ, et al. Burden of adhesions in abdominal and pelvic surgery: systematic review and met-analysis. BMJ 2013;347:f5588.

9. Schreinemacher MH, Emans PJ, Gijbels MJ, Greve JW, Beets GL, Bouvy ND. Degradation of mesh coatings and intraperitoneal adhesion formation in an experimental model. Br J Surg 2009;96:305-13.

10. Vogels RR, van Barneveld KW, Bosmans JW, et al. Long-term evaluation of adhesion formation and foreign body response to three new meshes. Surg Endosc 2015;29:2251-9.

11. Zhu LM, Schuster P, Klinge U. Mesh implants: An overview of crucial mesh parameters. World journal of gastrointestinal surgery 2015;7:226-36.

12. Schreinemacher MH, van Barneveld KW, Dikmans RE, Gijbels MJ, Greve JW, Bouvy ND. Coated meshes for hernia repair provide comparable intraperitoneal adhesion prevention. Surg Endosc 2013;27:4202-9.

13. Peeters E, van Barneveld KW, Schreinemacher MH, et al. One-year outcome of biological and synthetic bioabsorbable meshes for augmentation of large abdominal wall defects in a rabbit model. The Journal of surgical research 2013;180:274-83.

14. Gruber-Blum S, Brand J, Keibl C, et al. The impact of hydrophobic hernia mesh coating by omega fatty acid on atraumatic fibrin sealant fixation. Hernia : the journal of hernias and abdominal wall surgery 2015;19:651-7.

15. Diamond MP, Linsky CB, Cunningham T, Constantine B, diZerega GS, DeCherney AH. A model for sidewall adhesions in the rabbit: reduction by an absorbable barrier. Microsurgery 1987;8:197-200.

16. Zuhlke HV, Lorenz EM, Straub EM, Savvas V. [Pathophysiology and classification of adhesions]. Langenbecks Arch Chir Suppl II Verh Dtsch Ges Chir 1990:1009-16.

17. Dubcenco E, Assumpcao L, Dray X, et al. Adhesion formation after peritoneoscopy with liver biopsy in a survival porcine model: comparison of laparotomy, laparoscopy, and transgastric natural orifice transluminal endoscopic surgery (NOTES). Endoscopy 2009;41:971-8.

18. Claudio RH, Diogo Filho A, Mamede Filho DO. [Peritoneostomy with latex coated polypropylene: experimental study in rats]. Acta cirurgica brasileira / Sociedade Brasileira para Desenvolvimento Pesquisa em Cirurgia 2006;21:402-8.

19. Schreinemacher M, Henatsch D, van Barneveld K, Bouvy N. The need for standardised animal models and scoring systems in assessing mesh biocompatibility. Hernia : the journal of hernias and abdominal wall surgery 2010;14:335-6.

20. Dalkey NC. Delphi. Santa Monica, CA: RAND Corporation 1967.

21. Fitch K, Bernstein SJ, Aguilar MD, et al. The RAND/UCLA Appropriateness Method User's Manual. Santa Monica, CA: RAND Corporation 2001. 
Chapter 10

22. Moossdorff M, van Roozendaal LM, Strobbe $L$, et al. Maastricht Delphi consensus on event definitions for classification of recurrence in breast cancer research. Journal of the National Cancer Institute 2014;106.

23. ten Broek RP, Strik C, Issa Y, Bleichrodt RP, van Goor H. Adhesiolysis-related morbidity in abdominal surgery. Ann Surg 2013;258:98-106. 


\section{Chapter}

\section{Complications and recurrence rates of patients with Ehlers-Danlos syndrome undergoing ventral hernioplasty}




\section{ABSTRACT}

Background: Ventral hernia repair is one of the most frequently performed surgical procedures, though recurrences are common. Recurrence can be caused by impaired collagen formation or maturation, hence patients with Ehlers-Danlos syndrome (EDS) are potentially at increased risk for hernia recurrence. EDS causes altered collagen metabolism, though little is known about the influence of EDS on ventral hernioplasty outcomes. This study aims to analyse these patients to report complication rates, recurrence rates, and, if possible, to give recommendations for surgical intervention.

Methods: A retrospective analysis between January 2000 and January 2017 was performed in a university hospital Belgium (UZ Ghent). Data on baseline characteristics, primary surgery, and hernias was extracted from patients' medical charts. Noted endpoints were postoperative complications and recurrences.

Results: Fourteen patients (50\% males) were included. Ten (71\%) had an incisional hernia and four (29\%) a primary ventral hernia. Median age was 45 years (IQR 37.7552.75), median BMI was 24.82 (IQR 22.43-26.87). Four patients (29\%) smoked, one patient (7.1\%) had diabetes mellitus, and five patients (36\%) had an aneurysm of the abdominal aorta. All patients underwent elective open hernioplasty with mesh reinforcement. Three patients (21\%) had a postoperative complication (two infections, one seroma). Recurrence rate was $7.1 \%$ (one patient).

Conclusion: This series describes 14 patients with a median follow-up of 50 months and a recurrence rate of $7.1 \%$. The low recurrence rate could be explained by the use of large meshes that reinforce the entire midline to compensate for the reduced collagen strength in EDS patients. 


\section{INTRODUCTION}

Incisional hernia formation is one of the most frequent complications after abdominal surgery with midline laparotomy, occurring in $11 \%$ to $20 \%$ of all laparotomies in the general population. ${ }^{1}$ When patients have risk factors (obesity, smoking or abdominal aortic aneurysm), this rate can increase up to $35 \%{ }^{2}$

It is hypothesized that a disturbed balance between mature and immature collagen can be part of the underlying mechanism leading to incisional hernia formation. Klinge et al. explains recurrent hernia formation as a problem of biology and technique combined. ${ }^{3}$ The human extracellular matrix consists of twenty different types of collagen, of which 95\% is type I and III collagen. ${ }^{4}$ Patients with recurrent ventral hernias have a decreased collagen I/III ratios. ${ }^{3}$ Collagen type one is mature mechanically stable collagen, whereas collagen type III is immature, mechanically instable collagen. ${ }^{3}$ Alongside the previously mentioned collagens it has been hypothesizes by Schumpelick et al. that Tenascins, a family of glycoproteins, could be linked to hernia formation. ${ }^{5}$

EDS was first described 1901, the syndrome characterizes itself by a triad of skin hyperextensibility, joint hypermobility, and tissue fragility. Originally, EDS was divided into numbered subtypes. In 1998, the Villefranche classification scheme divided EDS into six subtypes, based on clinical features, biochemical and genetic findings, and mechanism of inherence: classic (type I and II), hypermobility (type III), vascular (type IV), kyphoscoliosis (type VI), arthrochalasia (type VIIA and VIIB), and dermatosparaxis (type VIIC). ${ }^{6,7}$ Because of overlapping symptoms in these different subtypes, categorizing EDS is no easy task. Including all subtypes of EDS, the incidence is approximately one in $\mathbf{5 0 0 0}$ people, of which the hypermobility subtype is most common. ${ }^{6}$

The recently published international classification of the Ehlers Danlos Syndrome describes the genetic basis for each type of EDS. The classical, vascular and arthrochalasie types have been liked to either type I or type III collagen disorders. ${ }^{4,8}$ The hypermobility type is linked to tenascin $X$ alterations. Although not all types of EDS have been linked to a specific protein disorders, many surgeons fear a high recurrence rate following hernia repair in EDS patients because of similar collagen disorders associated with both EDS and hernia recurrence. ${ }^{5,9,10}$

EDS can potentially influence every part of the body where connective tissue is present. The literature on the relationship between EDS and hernia development is scarce and only includes a few case reports. Despite the lack of evidence, many surgeons believe that EDS may have a negative effect on the clinical outcome of ventral hernioplasty in terms postoperative complications and recurrence rates. The aim of this retrospective case series is to evaluate outcomes of ventral hernioplasty in patients with EhlersDanlos syndrome, the primary outcome is hernia recurrence and the secondary outcome is postoperative complications. 


\section{METHODS}

A retrospective analysis of hospital registries between January 2000 and January 2017 was performed in a large university hospital in Ghent, Belgium (Ghent University Hospital). Before commencement of the study, ethical approval and approval of the Institutional Review Boards was obtained. The hospital central registry was searched using either ICD-10/ICD-9-CM or Diagnosis Treatment Codes (DBC) for collagen disorder (Q79.6/756.83) and 'hernioplasty' (0303.123/0303.124). Any patient with a history of EDS (any type) and a ventral abdominal hernioplasty was eligible for inclusion in this study. Patients that registered an objection for participation in scientific research in their medical chart were excluded. Follow-up was obtained from patients medical charts. All EDS patients are seen three weeks postoperative, as well as every six months hereafter.

\section{Data collection}

The following data were extracted from patients medical charts: baseline characteristics (age, sex, body mass index (BMI), smoking, medical history and type of Ehlers-Danlos), information about the hernia (date of diagnosis, type of hernia, size, primary/recurrent hernia, complaints), details regarding the surgical procedure (date of operation, elective/emergency procedure, type of procedure, mesh type and size and drain placement), postoperative data (postoperative complications (seroma, hematoma, surgical site infection, other infection, mesh explantation, and recurrence)), and follow-up (duration, number of outpatient clinic visits, readmissions, reoperations, and complaints). Hernia characteristics were reported using the European Hernia Classification of the European Hernia Society (EHS). ${ }^{11}$ All data was stored and analyzed in SPSS ${ }^{\circledR}$ for windows version 24, IBM corp. Armonk, NY, released 2013.

\section{RESULTS}

A total of 14 patients (seven males, seven females), with a median age of 45 years (range 24-60 years) were included with diagnosis dates between June 2009 and July 2016. Median BMI was 24.82 (IQR 22.43-26.87). Four patients smoked (29\%), one patient $(7.1 \%)$ had diabetes mellitus, and five patients (36\%) had an aneurysm of the abdominal aorta. Ten patients (71\%) were ASA Class II and three patients (21\%) were ASA Class III. Two patients (7.1\%) had the classic type Ehlers-Danlos, six patients (43\%) had the hypermobility type, and four patients (29\%) had the vascular type. Patient baseline characteristics are shown in Table 1. 
Table 1 Demographic characteristics

\begin{tabular}{|c|c|}
\hline & $\mathrm{n}=14$ \\
\hline Age at operation, years (IQR) & $45(38-53)$ \\
\hline Male (\%) & $7(50)$ \\
\hline $\mathrm{BMI}, \mathrm{kg} / \mathrm{m}^{2}(\mathrm{IQR})$ & $24.82(22.43-26.87)$ \\
\hline Smoking (\%) & $4(29)$ \\
\hline Diabetes Mellitus (\%) & $1(7.1)$ \\
\hline Abdominal Aortic Aneurysm (\%) & $5(36)$ \\
\hline \multicolumn{2}{|l|}{ ASA Class (\%) } \\
\hline 1 & $0(0)$ \\
\hline$\|$ & $10(71)$ \\
\hline III & $3(21)$ \\
\hline IV & $0(0)$ \\
\hline Unknown (\%) & $1(7.1)$ \\
\hline \multicolumn{2}{|l|}{ Ehlers-Danlos type (\%) } \\
\hline Classic (type I and II) & $2(7.1)$ \\
\hline Hypermobility (type III) & $6(43)$ \\
\hline Vascular (type IV) & $4(29)$ \\
\hline Kyphoscoliosis (type VI) & $0(0)$ \\
\hline Arthrochalasia (type VIIA and B) & $0(0)$ \\
\hline Dermatosparaxis (type VIIC) & $0(0)$ \\
\hline Unknown (\%) & $2(14)$ \\
\hline \multicolumn{2}{|c|}{ Type of primary surgery (in case of incisional hernia, $n=10$ ) } \\
\hline Gynecologic & 2 \\
\hline Vascular & 4 \\
\hline Gastric & 3 \\
\hline Colorectal & 1 \\
\hline
\end{tabular}

IQR, interquartile range; BMI, body mass index; ASA, American Society of Anesthesiologists

\section{Hernia characteristics}

Ten patients (71\%) had an incisional hernia and four patients (29\%) had a primary hernia (see Figure 1 for hernia characteristics). One of the incisional hernias was a recurrent hernia, previously treated with a Marlex ${ }^{\circledR}$ mesh. The median hernia length of the primary hernias was $2.5 \mathrm{~cm}$ (range 2.0-5.0 cm), and the median width was $2.0 \mathrm{~cm}$ (range 1.5-3.0 cm). The median hernia length of the incisional hernias was $5.0 \mathrm{~cm}$ (range 0.6-25 cm), and the median width was $3.5 \mathrm{~cm}$ (range 0.8-15 cm). None of the patients underwent any concomitant procedures, nor had they concomitant hernias in other locations. 


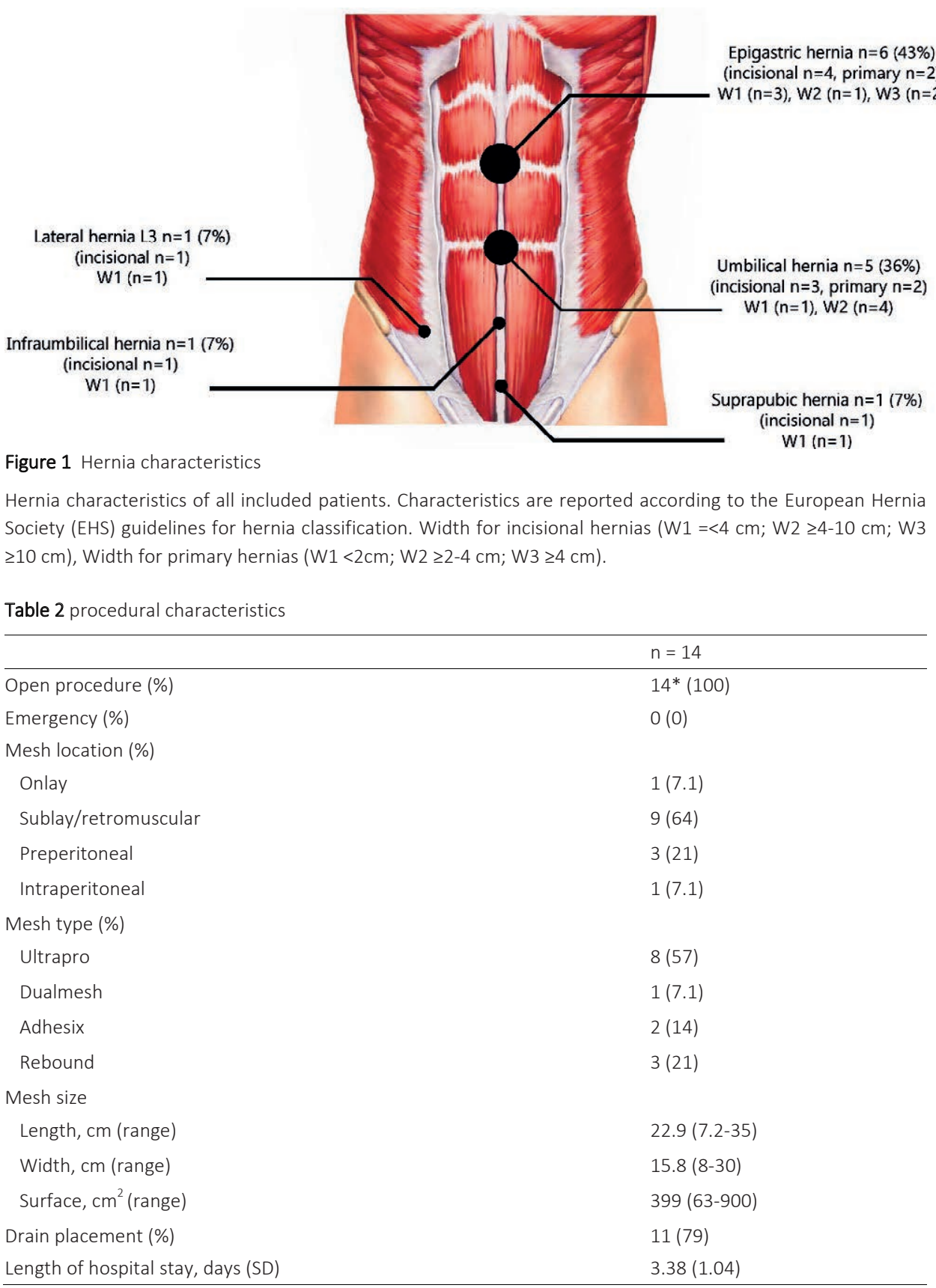

*One procedure started as a laparoscopic procedure, but was converted to an open procedure; Surface of every mesh was calculated as length* width; SD= standard deviation 


\section{Surgical characteristics}

All hernia repair procedures were elective (see Table 2 for operation details). In all patients open surgery was performed, one procedure (a recurrent hernia repair) was planned as a laparoscopic procedure though converted to an open procedure because of severe adhesions in the abdominal cavity. All patients received mesh reinforcement in either onlay $(n=1,7 \%)$, sublay $(n=9,64 \%)$, preperitoneal $(n=3,21 \%)$, or intraperitoneal $(n=1,7 \%)$ position. The onlay procedure was performed using an Adhesix mesh, sublay repairs were performed with UltraPro $(n=7)$, Adhesix $(n=1)$, or Rebound ${ }^{\circledR}(n=1)$ mesh. Preperitoneal repairs were performed using the Ultrapro $(n=1,33 \%)$, or Rebound $^{\circ}(n=2,67 \%)$ mesh, and the intraperitoneal procedure was performed using a Dualmesh ${ }^{\circledR}$. Average mesh size was $399 \mathrm{~cm}^{2}$ (range $63-900 \mathrm{~cm}^{2}$ ), with an average length of $23 \mathrm{~cm}$ (range 7.0-35 cm), and width of $16 \mathrm{~cm}$ (range $8.0-30 \mathrm{~cm}$ ). All sublay repairs could be closed in the midline using the Rives-Stoppa technique without the need for additional procedures (component separation or other). Eleven patients (79\%) received a drain at the end of the procedure.

\section{Perioperative outcomes}

Table 3 perioperative complications

\begin{tabular}{ll}
\hline & $\mathrm{n}=14$ \\
\hline No complications (\%) & $11(79)$ \\
Seroma (\%) & $1(7.1)$ \\
Hematoma (\%) & $0(0)$ \\
Surgical Site Infection (\%) & $2(14)$ \\
Other infection (\%) & $0(0)$ \\
Mesh explantation (\%) & $0(0)$ \\
Other (\%) & $0(0)$ \\
\hline
\end{tabular}

* One procedure started as a laparoscopic procedure, but was converted to an open procedure; Surface of every mesh was calculated as length* width; SD= standard deviation

No intra-operative complications were recorded. Postoperative complications occurred in three patients (21\%) (Table 3 ). Mean hospital stay was 3.4 days ( \pm 1.04 days). One patient $(7 \%)$ had a seroma and two patients (14\%) had a surgical site infection. One of the patients with a surgical site infection required antibiotic treatment and was therefore classified as a Clavien-Dindo Grade II complication. ${ }^{12}$ The remaining two complications were Grade I. No other complications were recorded in the 30-day postoperative period. 


\section{Long term outcomes}

Median follow-up after surgery was 50 months (IQR 18-82, range 6-152 months). Patients visited the outpatient clinic a mean of three times (range 1-4 times). During this follow-up period, recurrences were assessed using clinical examination $(n=3,29 \%)$, or clinical examination combined with ultrasonography $(n=10,71 \%)$. One patient $(7 \%)$ developed a hernia recurrence. This patient was a 37-year-old female with EDS type III without relevant comorbidity or medical history (no diabetes, no aortic aneurysm, no smoking). The patient's BMI was 25.7. She presented with a primary umbilical hernia of $2 \times 2 \mathrm{~cm}$ (EHS primary abdominal wall hernia classification: midline epigastric medium hernia). She underwent an elective open repair with a preperitoneal mesh placement (Rebound $9 \times 8 \mathrm{~cm}$ ) in August 2009. The procedure was without any complication. She developed a clinical recurrence after approximately 24 months follow-up, which resulted in aesthetic complaints. The patient did not seek medical attention for her recurrence until 89 months follow-up, as she does not wish to undergo reoperation for the recurrence. No readmissions were performed. During follow-up one patient died due to brain haemorrhage, unrelated to the hernioplasty procedure. For this patient, no longterm follow-up was available.

\section{DISCUSSION}

This case series of 14 patients with Ehlers-Danlos syndrome (EDS) undergoing ventral hernia repair shows a $7.1 \%$ recurrence rate after a median follow-up period of 50 months. Current literature on ventral hernioplasty in EDS patients is scarce with only a hand full of case reports. Girotto et al. describes two patients with EDS and recurrent ventral abdominal wall hernias. ${ }^{13}$ He treated these patients with a components separation technique and an onlay Marlex mesh. Follow-up in this study is not described clearly. Fogel et al. describes a series of six ventral hernia repairs, of whom two patients got a recurrent hernia, though important details regarding the surgical procedure and follow-up are not described as the focus of the article is on EDS and not on ventral hernia repair.

The current series is the first study that looks at patients with EDS as a specific risk group for developing hernia recurrence. Even though the detailed pathophysiology of incisional hernia formation is still illusive, many factors influence surgical wound healing and ultimately hernia formation. ${ }^{14}$ One important factor is collagen synthesis and maturation. Given the well-established collagen impairment in EDS patients, a high recurrence rate in in this population was anticipated. However, the 7\% recurrence rate after open mesh repair in this study is lower than the $12 \%$ found in the general patient population after elective open ventral hernioplasty with mesh reinforcement, after a median follow-up of 59 months. ${ }^{15}$ The low recurrence rate can potentially be explained by the 
large mesh size. The average mesh size in this series was $16 \times 23 \mathrm{~cm}$, for an average hernia size of $3 \times 5 \mathrm{~cm}$. The 'oversized' mesh ensured a large surface for tissue ingrowth, which could compensate for the reduced collagen quality.

During the 17-year inclusion period of this consecutive case series only 14 patients were identified. There are several explanations for the relatively low number of EDS patients with ventral hernias. The most obvious one would be the low prevalence of EDS (1:5000). Another, more troubling, explanation would be identification failure of EDS in the outpatient hernia clinic. Since this case series is one of the first articles to discuss the potential influence of EDS on ventral hernia surgery outcomes, the problem may be underestimated or overlooked. Since EDS may be 'diagnosed' by one of many physicians either inside, or outside the hospital, central hospital registries may not always be up-to-date concerning the patient history. Hence the treating physician must actively acquire information regarding EDS symptoms, to not overlook the disease in the outpatient hernia clinic.

\section{Limitations}

A retrospective case series is methodologically unsuitable to determine the recurrence rate of ventral hernias in EDS patients accurately, hence the percentages reported in this series must be interpreted with caution. Furthermore, because of the small sample size, detailed analysis of the relations between different types of EDS and clinical outcomes could not be made. Despite the abovementioned limitation, it is the authors' opinion that the following conclusions can be made based on the results of this article.

\section{Recommendations for hernioplasty in EDS patients}

- Establishing the diagnosis Ehlers-Danlos syndrome is the first step in providing tailored care for this complex patient population. If the family history or physical examination suggests Ehlers-Danlos syndrome, further examination is advised before attempting ventral abdominal wall hernioplasty.

- Treat 'small' ventral abdominal wall hernias as if they were bigger. The patients described in this series presented with relatively 'small' ventral hernias, though they were treated with a large (oversized) mesh and an extensive repair (most often Rives-Stoppa) with reinforcement along the entire midline or previous incision. Using large meshes provides a large surface for tissue ingrowth, which could compensate for the collagen impairment in EDS patients. 


\section{CONCLUSION}

Patients with EDS are prevalent in the ventral abdominal wall hernia population. Identifying these patients is the first step towards tailored care. This series describes 14 patients with a median follow-up of 50 months and a recurrence rate of $7.1 \%$ (one patient). The low recurrence rate observed in this series might be explained by the use of a large mesh and reinforcement of the entire midline to compensate for the reduced collagen strength in EDS. 


\section{REFERENCES}

1. Deerenberg EB, Harlaar JJ, Steyerberg EW, et al. Small bites versus large bites for closure of abdominal midline incisions (STITCH): a double-blind, multicentre, randomised controlled trial. Lancet 2015;386: 1254-60.

2. Muysoms FE, Detry O, Vierendeels T, et al. Prevention of Incisional Hernias by Prophylactic Meshaugmented Reinforcement of Midline Laparotomies for Abdominal Aortic Aneurysm Treatment: A Randomized Controlled Trial. Ann Surg 2016;263:638-45.

3. Henriksen NA. Systemic and local collagen turnover in hernia patients. Dan Med J 2016;63.

4. Antoniou GA, Georgiadis GS, Antoniou SA, Granderath FA, Giannoukas AD, Lazarides MK. Abdominal aortic aneurysm and abdominal wall hernia as manifestations of a connective tissue disorder. Journal of vascular surgery 2011;54:1175-81.

5. Rosch R, Junge K, Knops M, Lynen P, Klinge U, Schumpelick V. Analysis of collagen-interacting proteins in patients with incisional hernias. Langenbecks Arch Surg 2003;387:427-32.

6. Beighton P, De Paepe A, Steinmann B, Tsipouras P, Wenstrup RJ. Ehlers-Danlos syndromes: revised nosology, Villefranche, 1997. Ehlers-Danlos National Foundation (USA) and Ehlers-Danlos Support Group (UK). Am J Med Genet 1998;77:31-7.

7. Callewaert B, Malfait F, Loeys B, De Paepe A. Ehlers-Danlos syndromes and Marfan syndrome. Best Pract Res Clin Rheumatol 2008;22:165-89.

8. Muysoms FE, Miserez M, Berrevoet F, et al. Classification of primary and incisional abdominal wall hernias. Hernia : the journal of hernias and abdominal wall surgery 2009;13:407-14.

9. Dindo D, Demartines N, Clavien PA. Classification of surgical complications: a new proposal with evaluation in a cohort of 6336 patients and results of a survey. Ann Surg 2004;240:205-13.

10. Girotto JA, Malaisrie SC, Bulkely G, Manson PN. Recurrent ventral herniation in Ehlers-Danlos syndrome. Plastic and reconstructive surgery 2000;106:1520-6.

11. Franz MG. The Biology of Hernia Formation. The Surgical clinics of North America 2008;88:1-vii.

12. Kokotovic D, Bisgaard T, Helgstrand F. Long-term Recurrence and Complications Associated With Elective Incisional Hernia Repair. JAMA 2016;316:1575-82. 

Chapter

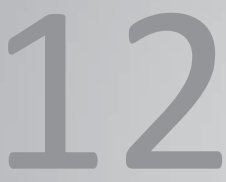

Sex differences in baseline performance of the ischemic button model for induction of adhesions in laboratory rats

\author{
Elwin H.H. Mommers ${ }^{1}$, Liu Hong ${ }^{1}$, Audrey Jongen ${ }^{1}$, Nicole D. Bouvy ${ }^{1}$ \\ ${ }^{1}$ Department of Surgery, Maastricht University Medical Center, Maastricht, The Netherlands
}

Submitted to 'Laboratory Animals' 


\section{ABSTRACT}

Background: The ischemic button model is frequently used for the induction of adhesions in laboratory animals. Despite the frequent use of the model, a sex comparison has never been performed. Sex comparisons are an important aspect of refining animal experiments and provide evidence-based data regarding baseline performance of the model. The aim of this study is to compare baseline performance of the modified ischemic button model in male and female Wistar Han rats.

Methods: Twenty-four adult Wistar Han rats (12 male, 12 female), aged eight weeks (weight range male 200-250 g, female 150-175 g) bred by Charles River Laboratories Inc. were included in this study with a follow-up of one week. Noted endpoints were: intra-abdominal adhesions formation scored with the Zühlke score and a 14-item animal welfare assessment.

Results: A total of 192 ischemic buttons (96 male/ 96 female) were created. After one week follow-up 93 buttons survived in the male group (96.9\%) versus 91 in the female group (94.8\%) ( $p=0.409)$. In the male group 85/93 (91.4\%) buttons induced adhesions versus $84 / 91$ (92.3\%) in the female group ( $p=0.881$ ). All but one animal was classified Zühlke score 3. No significant difference was observed in the postoperative welfare scores, though male animals increased significantly faster in weight compared to females $(p<0.001)$.

Conclusion: The ischemic button model results in equal quality and quantity of intraabdominal adhesions in male and female Wistar Han rats. Male rats gain weight faster after the operation, though both male and female rats are suitable for the induction of experimental adhesions. 


\section{INTRODUCTION}

Intra-abdominal postoperative adhesions are fibrotic connections between intraabdominal organs and structures that are normally unconnected. ${ }^{1}$ The adhesions are the results of a pathological healing following inflammation of the peritoneum and occur after nearly $100 \%$ of all intra-abdominal surgical procedures. ${ }^{2,3}$ Approximately 5$18 \%$ percent of the adhesions will become clinically relevant and cause intestinal obstruction, infertility, or chronic abdominal pain. ${ }^{1,4,5}$ Due to the vast number of intraabdominal procedures performed every year, intra-abdominal adhesions pose a billiondollar burden on the global healthcare system. ${ }^{1,3-7}$ As a consequence, the development and evaluation of anti-adhesive components is a large industry that depends heavily on the use of experimental animal models that provide a consistent induction of experimental adhesions. The ischemic button model is one of the most frequently used animal models for experimental adhesions. In short, the technique comprises of a suture tied around a $5 \mathrm{~mm}$ portion of the parietal peritoneum. Subsequently, the ligated tissue becomes ischemic, resulting in the development of an adhesion. The original model described by Buckenmaier et al. is susceptible to failure of the buttons due to slipping of the sutures. ${ }^{8}$ To improve the original technique, Rajab et al. introduced a modified ischemic button model in $2011 .^{9}$ Rajab et al. improved to model by introducing a backstitch ligature to reduce the number of slipped sutures and create durable ischemic buttons. In their validation article they compared the modified ischemic button model to the original model and found a lower percentage of button obliteration in the modified ischemic button model (18.8\% vs $3.8 \%) .{ }^{9}$ Since the introduction of the modified technique, it has been applied multiple times in both male and female rats, though most studies concern male rats, possibly because they have larger fat depositions in the lower abdomen (epidydimal or peri-testicular fat) that can facilitate adhesion formation. Despite the frequent use of the ischemic button model in animal experiments, a comparison in baseline performance between male and female rats has never been performed. Comparing baseline performance between both sexes is an important aspect of refining animal experiments and provides evidence-based data regarding the number and sex of the animals included in future research. The aim of this study is to compare baseline performance of the modified ischemic button model in both male and female Wistar Han rats.

\section{METHODS}

Before commencement of this study, ethical approval was obtained from the Dutch Central Committee of Animal experiments and from the institutions review board, Animal Ethical committee, and Inspection for Animal Welfare (Licence number AVD107002016720). This study was performed according to the European Convention 
for the Protection of Vertebrate Animals used for Experimental and other Scientific Purposes (ETS No.123)

Twenty-four adult Wistar Han rats, twelve male and twelve females, aged eight weeks (weight range male 200-250 g, female 150-175 g) and bred by Charles River Laboratories incorporated (Sulzfeld, Germany) were socially housed in EU type IV MacroIon cage of $1800 \mathrm{~cm} 2$ with cage enrichment in the form of a cardboard cylinder and a wooden block at the centralised animal experiments facility of the Maastricht University Medical Center in Maastricht, The Netherlands. Rooms were fitted with an artificial 12hour light-dark cycle, room temperature was standardised at $25{ }^{\circ} \mathrm{C}$, food and water were provided ad libitum in the form of sterilized compressed food pellets $(10 \mathrm{~mm}$ Sniff rat/mouse maintenance food) and acidified drinking water. All animals were followed for seven days before being euthanized under general anaesthesia via cardiac puncture.

\section{Procedure}

All operations were performed by two surgeons (EM, LH) certified for working with laboratory animals. All animals were randomly assigned to one of the two surgeons via a 2x12 block randomization list (Sealed Envelope Ltd. 2016). Buprenorphine (0.05mg/kg; Buprecare AST Pharma) and Carprofen (4 mg/kg; Norocarp ${ }^{\oplus}$ Norbrook laboratories) were administered subcutaneously as preoperative analgesia. Anaesthesia was induced using 3-4 vol.\% Isoflurane (IsoFlo Abbott laboratories), and maintained with 2-3 vol.\%. Animals were placed on a heating pad of $38^{\circ} \mathrm{C}$ and their temperature was monitored using a rectal probe. The abdominal fur was removed with electric clippers. The ischemic button model was applied according to the original publication of Rajab et al. ${ }^{9}$. In short, the technique comprises of a midline incision and the creation of four paramedian ischemic buttons on both sides of the abdominal wall (eight buttons/animal), placed $1 \mathrm{~cm}$ lateral of the midline incision and spaced $1 \mathrm{~cm}$ apart. Each button was created by grabbing a small $5 \mathrm{~mm}$ portion of parietal peritoneum and ligating the base of the tissue with a non-absorbable suture (4-0 Prolene Ethicon). After tying the suture, it was turned around the entire base of the tissue and tied again to create the ischemic button. This procedure was repeated for all eight buttons. Hereafter, the abdomen and the skin are closed in the midline using absorbable sutures (4-0 Polyglactin 910 Vycril Ethicon and 4-0 poliglecaprone 25 Monocryl Ethicon respectively). The animals were placed in a heated recovery cage (temperature $\approx 36^{\circ} \mathrm{C}$ ) ventilated with $100 \%$ oxygen $1 \mathrm{~L}$ per minute for postoperative recovery. Buprenorphine $0.03 \mathrm{mg} / \mathrm{kg}$ was given 12 hours after the pre-operative analgesia. The morning of the first postoperative day, Carprofen $4 \mathrm{mg} / \mathrm{kg}$ was given to all animals. Hereafter, analgesia was administered based on clinical signs of distress. 


\section{Outcome measurement}

The primary outcome of the study was intra-abdominal adhesion formation. The quantitative analysis of adhesion formation was scored by calculating the amount of intraabdominal adhesions formed to the number of remaining buttons after one week follow-up (eight buttons per animal - obliterated buttons due to postoperative mobilisation). Standardised scoring of the adhesion was based on standardised pictures taken by the surgeons (figure 1) and performed by two researchers (EM, LH) blinded for sex. Qualitative analysis of the adhesions was performed using the macroscopic Zühlke score (table 1). ${ }^{10}$ Secondary endpoints were sex-based differences in welfare scores, postoperative weight gain, need of postoperative analgesia, and inter-surgeon variability. Welfare was assessed using a standardised welfare scoring sheet. Each animal's condition was scored twice daily for the first two postoperative days, hereafter each animal was scored once a day. A welfare assessment comprised of scoring the following items; weight, activity, behaviour, gait, posture, body condition, fur, hydration, breathing, faeces/urine, mucosa/extremity, surgical wound, oedema, necrosis. Each item was scored 0 (no abnormality) to 3 (very abnormal).

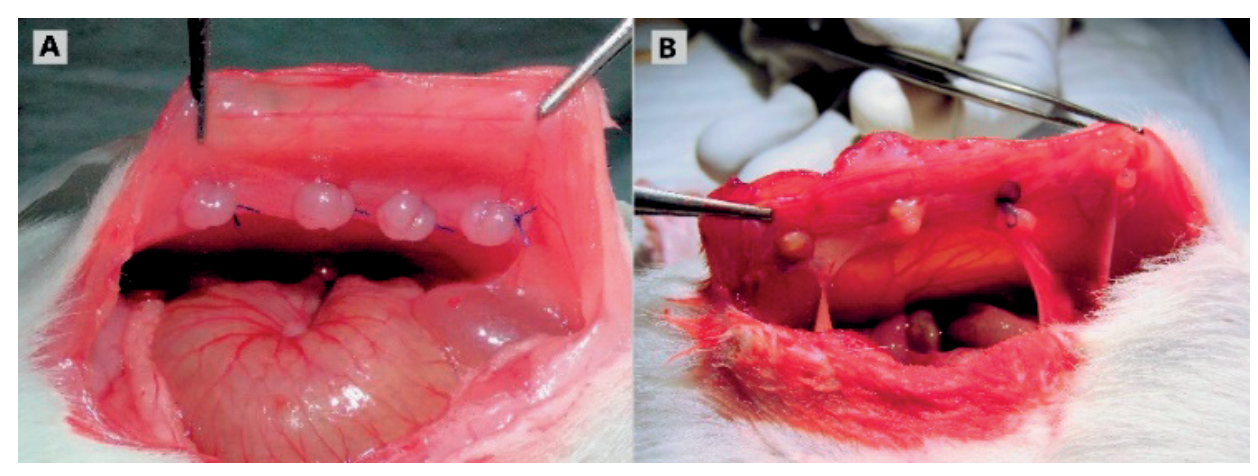

Figure 1 Pre- and postoperative view of ischemic buttons

$A=$ preoperative view of four ischemic buttons; $B=$ postoperative view of four ischemic buttons with adhesions to omentum and epidydimal/peri-testicular fat.

Table 1 Zühlke macroscopic adhesion score

\begin{tabular}{ll}
\hline Grade 0 & No adhesions/ insignificant adhesions \\
\hline Grade 1 & Only one adhesions band between the organs or between one organ and abdominal wall \\
Grade 2 & $\begin{array}{l}\text { Two adhesions bands between organs or between one organ and abdominal band } \\
\text { Grade } 3\end{array}$ \\
$\begin{array}{l}\text { More than two adhesion bands between the organs or between one organ and abdominal wall } \\
\text { or adhesions of intestinal loops without any adhesion to the abdominal wall }\end{array}$ \\
Grade 4 & Adhesions of all viscera to the abdominal wall \\
\hline
\end{tabular}

Zühlke's macroscopic adhesions scoring system; (-)= not applicable. Score adapted from Zühlke et al. Pathophysiology and classification of adhesions. Langenbecks Arch. Chir. Suppl. II Verh. Dtsch. Ges. Chir 1990;16:1009-16. 


\section{Statistical analysis}

Based on an alfa 0.05, beta 0.20, and effect size 2.0, 12 rats were needed in each group. Statistical analysis for the outcomes 'adhesions' and 'welfare/analgesia' were performed using SPSS version 22, SPSS Statistics for Windows, IBM corp. Armonk, NY, released 2013. Analysis of weight gain and visual representation of delta weight gain was analysed/computed using GraphPad Prism version 6.00 for Windows, GraphPad Software, La Jolla California USA, www.graphpad.com. Any group comparison was performed using independent student T-tests or Mann-Whitney- $U$ tests depending on the distribution of the data (F-test).

\section{RESULTS}

Two male rats had wound dehiscence in the middle of the laparotomy wound with an intact underlying fascia. These animals were re-operated on the first postoperative day, the wound was cleaned and the edges of the wound were trimmed until punctate bleeding was observed. Hereafter, the abdomen was closed intra-cutaneous using 4-0 Monocryl sutures.

Table 2 Adhesion tissue

\begin{tabular}{lll}
\hline & Males & Females \\
\hline Omentum & 41 & 42 \\
Epidydimal/parametrial fat & 40 & 37 \\
Pancreas & 2 & 3 \\
Liver & 2 & 0 \\
Stomach & 0 & 1 \\
Bladder & 0 & 1 \\
Total & 85 & 84 \\
\hline
\end{tabular}

Anatomical structures that adhered to the ischemic buttons.

\section{Adhesions}

In total 192 buttons (96 male/ 96 female) were created during this study. After one week follow-up 93 buttons survived in the male group (96.9\%) versus 91 in the female group (94.8\%) ( $p=0.409)$. In the male group 85/93 (91.4\%) buttons induced adhesions versus $84 / 91(92.3 \%)$ in the female group $(p=0.881)$. In the male group, all but one animals were classified as Zühlke type 3, one animal was classified as Zühlke type 4. All female animals were classified as Zühlke type $3(p=0.339)$. See table 2 for anatomical structures that adhered to the ischemic buttons. The number of surviving buttons was equal for both surgeons. Variance in the number of adhesion on the remaining buttons 
was 1.174 vs 1.636 ( $p=0.865)$. There was no significant difference between the number of adhesions to the epidydimal or parametrial fat in the lower abdomen $(p=0.730)$.

\section{Welfare/analgesia}

Welfare scores did not differ statistically significant between male and females apart from the fourth postoperative day. At the fourth day, the mean overall welfare score for the male rats was 7.3 compared to 5.3 in the female group $(p=0.043)$. A higher score indicates more welfare impairment.

All animals received analgesia for the first 48 hours. Hereafter analgesia was provided based on the welfare scores of an individual animal and/or clinical signs of distress. Additional analgesia was administered nine times in de male group and eight times in the female group $(p=0.807)$. As mentioned before there were two male rats with a reoperation for wound dehiscence, if analgesia within the first 48 hours from reoperation are excluded from the analysis there was no statistically significant difference between male and female administration of analgesia $(p=0.797)$.

\section{Weight gain}

Weight was measured daily throughout the follow-up. A delta-weight gain was calculated by subtracting the baseline weight of each animal from the measured value to correct for sex-based differences at baseline. Male rats seemed to gain weight significantly faster compared to females (figure 2). At the end of follow-up $83 \%$ of the male rats had reached or exceeded the pre-operative weight compared to $33 \%$ of the female rats. Differences at postoperative day five, six, and seven are statistically significant (figure 2).

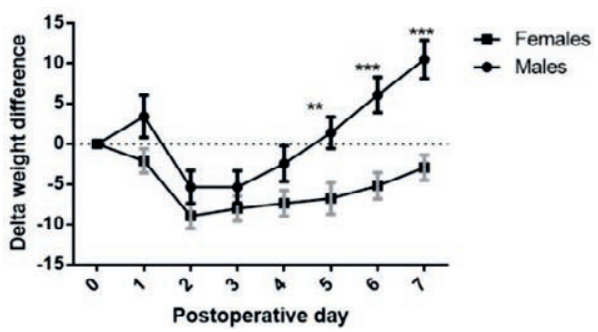

Figure 2 Delta weight gain during follow-up

Delta weight gain during follow-up for males and females. The dotted line reflects baseline weight. Whiskers $=$ standard error of the mean (SEM), $* *=p<0.01, * * *=p<0.001$. Postoperative day $0=$ references value at baseline.

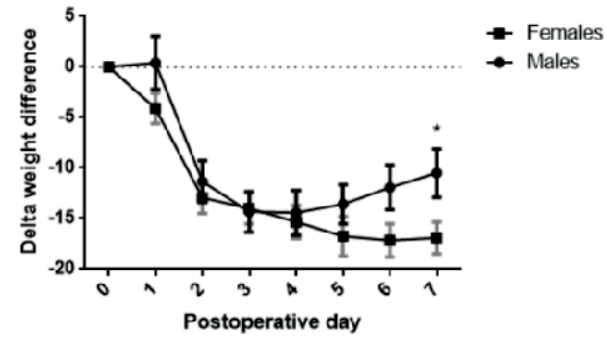

Figure 3 Delta weight gain corrected for physiological growth

The dotted line reflects a 'normal' weight for each timepoint based on growth data supplied by Charles River Laboratories concerning German bred Wistar Han rats of eight weeks old. Whiskers $=$ SEM, $\left({ }^{*}\right)=$ $p<0.05$; Statistical significance $6^{\text {th }}(p=0.068)$ and $7^{\text {th }}$ $(p=0.035)$ postoperative day. 
Figure 3 shows the delta weight gain corrected for normal physiological growth. The first six postoperative days did not differ significantly. Postoperative day seven shows a significant difference between males and females (-10.56 g vs $-16.9 \mathrm{~g}$ respectively, $p=0.068)$.

\section{DISCUSSION}

The ischemic button model is amongst the most frequently used models for the induction of experimental adhesions. ${ }^{11-15}$ This study shows there is no quantitative nor qualitative difference in the performance of the ischemic button model between male and female Whistar Han rats. In conjunction with the clinical performance, there appears to be no clinically relevant difference in welfare scores between male and female rats, nor was there a significant difference in the need for additional postoperative analgesia. Despite these similarities, male rats reached baseline weight significantly quicker compared to female rats. When the delta weight gain is corrected for physiological growth, the significantly faster weight gain remains statistically significant at the end of the follow-up. The faster weight gain in male rats can be explained by multiple factors, ranging from faster postoperative recovery in the male group, insufficient analgesia schemes in the female group, or unrecognised need for pain medication in the female group. ${ }^{16}$ Moreover, there is increasing evidence that pain perception has sex based differences. ${ }^{17}$ The 2015 publication of Sorge et al. concerning pain perception in male and female mice describes sexual dimorphism in pain perception based on Toll like receptor 4 and dorsal horn gene expression. ${ }^{17,18}$ Moreover, previous studies have demonstrated that opioids have a larger analgesic effect in male versus female rats. ${ }^{19,20}$ These findings suggest sexual dimorphism in pain perception, as well as pain analgesia.

Button obliteration due to slipping of the suture (3.1\% male group vs $5.2 \%$ female group) in this study was comparable to the study of Rajab et al. that reported a button obliteration of $3.8 \%$ using the modified method. ${ }^{9}$ Adhesion induction of the remaining studies is noticeably higher (91.4\% male group vs $92.3 \%$ female group) than previously published work such as the study of Rajab et al who reports $62.3 \%$ adhesions in the modified group and $69.2 \%$ adhesions in the original ischemic button group. Though adhesion induction in this model varies in published literature from $62.3 \%$ to $92 \%$. $^{9,12,13}$ Variation in button obliteration and adhesion induction can be explained by multiple factors such as surgeon performance, suture material, button size, duration of the procedure, and duration of follow-up. Button size refers to the amount of tissue protruding over the suture. When tying the back and front ligature of the ischemic button it is important to keep the angle between the suture and the abdominal wall as small as possible. This will ensure the creation of a large, durable ischemic button (figure 4).

The type of suture used in the ischemic button model varies from braided absorbable sutures to monofilament non-absorbable sutures. ${ }^{9,11-15}$ A suture comparison for the 
ischemic button model has not been performed, though each type of suture has proand disadvantages. Braided sutures are easier to tie though usually thicker compared to monofilament sutures. Silk or monofilament sutures are thinner and create a smaller base of the button with profound bulging of the tissue, though they are difficult to tie. Differences in histological response between absorbable and non-absorbable sutures may exist, though are currently based on speculation. ${ }^{21,22}$

A

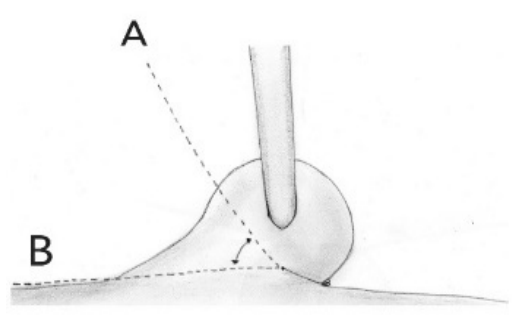

B
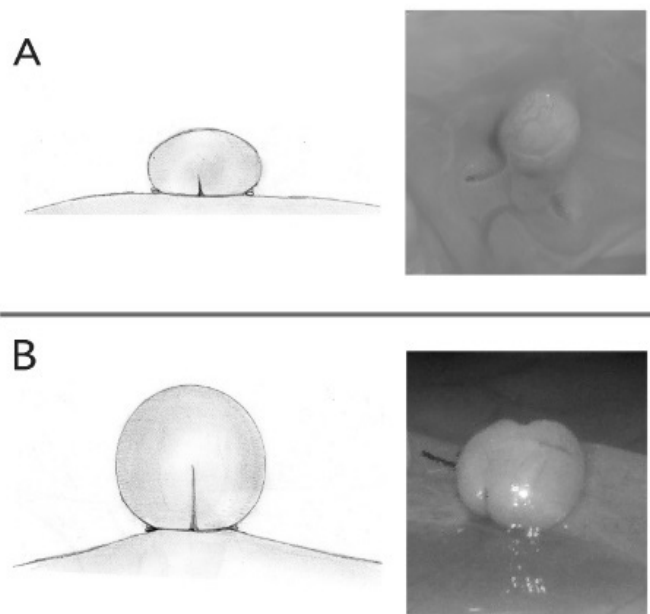

Figure 4 Making durable ischemic buttons

When tying the suture (after completing the backstitch) the angle between the suture and the abdominal wall (double headed arrow) can vary. A large angle, shown by suture line 'A' will result in a small button. A small angle, shown by suture line ' $B$ ' will result in a bigger, and more durable button.

The type tissue adhering to the buttons was comparable in male and female rats. Often male rats are used for this model due to their abundance of epidydimal fat that is prone to adhere to the buttons. This study demonstrates that there is no difference in the type or location of adhesion induction between male and female rats. Females may have smaller intra-abdominal (parametrial) fat storage, though this does not influence proneness to adhesion formation in the lower abdomen.

This study used an extensive 14-item welfare assessment, though this instrument has never been validated. Welfare in males and females was scored by the same observer to prevent inter-observer bias. Postoperative analgesia was standardised during the first 48 hours postoperative, though during the remained of follow-up analgesia usage differed between the groups depending on clinical/welfare assessments. Given the previously mentioned dimorphism in pain perception and analgesic efficacy of opioids, comparing welfare and pain perception between male and female rats is difficult, and attributing any observed difference in pain perception or analgesia treatment to a single cause is not possible in the current experiment. Despite these limitations, the following conclusion can be drawn from this study. 
Tips and tricks for making ischemic buttons

- The type of organ/tissue adhering to the ischemic button depends heavily on the location of the button. Hence, scoring the type organs or even 'organ involvement' seems unjust in this model.

- When making the button the angle between the suture and the abdominal wall must be as small as possible to create large, durable buttons (figure 4).

- Hold the suture knot for approximately five seconds during tying of the suture to allow the tied tissue to settle before tying the last knot to guaranty a tight ligature.

- Results of the ischemic button model vary in published literature, possibly due to surgeon performance, button size, type of suture material and differences in followup. Surgeon performance and button size can be perfected by experience, for instance in post-mortem animals.

\section{CONCLUSION}

The ischemic button model qualitative and quantitative performance is equal in male and female Whistar Han rats. Male rats seem to gain weight faster during follow-up and reach baseline weight sooner compared to female rats. There were no observed differences in postoperative welfare scores nor the need for additional analgesia between males and female rats. 


\section{REFERENCES}

1. Hellebrekers BW, Kooistra T. Pathogenesis of postoperative adhesion formation. Br J Surg 2011;98:150316. doi: 10.002/bjs.7657. Epub 2011 Aug 23.

2. Arung W, Meurisse M, Detry O. Pathophysiology and prevention of postoperative peritoneal adhesions. World J Gastroenterol 2011;17:4545-53.

3. Ouaïssi M, Gaujoux S, Veyrie N, et al. Post-operative adhesions after digestive surgery: Their incidence and prevention: Review of the literature. Journal of Visceral Surgery 2012;149:e104-e14.

4. Ellis H, Moran BJ, Thompson JN, et al. Adhesion-related hospital readmissions after abdominal and pelvic surgery: a retrospective cohort study. Lancet 1999;353:1476-80.

5. Wilson MS. Practicalities and costs of adhesions. Colorectal Dis 2007;9:60-5.

6. Kossi J, Salminen P, Rantala A, Laato M. Population-based study of the surgical workload and economic impact of bowel obstruction caused by postoperative adhesions. Br J Surg 2003;90:1441-4.

7. Ray NF, Denton WG, Thamer M, Henderson SC, Perry S. Abdominal adhesiolysis: inpatient care and expenditures in the United States in 1994. J Am Coll Surg 1998;186:1-9.

8. Buckenmaier CC, 3rd, Pusateri AE, Harris RA, Hetz SP. Comparison of antiadhesive treatments using an objective rat model. The American surgeon 1999;65:274-82.

9. Rajab TK, Wauschkuhn CA, Smaxwil L, Kraemer B, Wallwiener M, Wallwiener CW. An improved model for the induction of experimental adhesions. J Invest Surg 2010;23:35-9. doi: 10.3109/08941930903469474.

10. Zuhlke HV, Lorenz EM, Straub EM, Savvas V. [Pathophysiology and classification of adhesions]. Langenbecks Arch Chir Suppl II Verh Dtsch Ges Chir 1990;16:1009-16.

11. Cassidy MR, Sherburne AC, Heydrick SJ, Stucchi AF. Combined intraoperative administration of a histone deacetylase inhibitor and a neurokinin-1 receptor antagonist synergistically reduces intra-abdominal adhesion formation in a rat model. Surgery 2015;157:581-9.

12. Kraemer B, Wallwiener C, Rajab TK, Brochhausen C, Wallwiener M, Rothmund R. Standardised models for inducing experimental peritoneal adhesions in female rats. Biomed Res Int 2014;2014:435056.: 10.1155/2014/435056. Epub 2014 Apr 8.

13. Sheldon HK, Gainsbury ML, Cassidy MR, Chu DI, Stucchi AF, Becker JM. A sprayable hyaluronate/carboxymethylcellulose adhesion barrier exhibits regional adhesion reduction efficacy and does not impair intestinal healing. Journal of gastrointestinal surgery : official journal of the Society for Surgery of the Alimentary Tract 2012;16:325-33.

14. Vogels RR, Bosmans JW, van Barneveld KW, et al. A new poly(1,3-trimethylene carbonate) film provides effective adhesion reduction after major abdominal surgery in a rat model. Surgery 2015;157:1113-20.

15. Wallwiener M, Wallwiener CW, Molinas R, et al. Intraabdominal adhesion formation is associated with differential mRNA expression of metabolic genes PDHb and SDHa. Archives of gynecology and obstetrics 2012;286:683-6.

16. Waite ME, Tomkovich A, Quinn TL, et al. Efficacy of Common Analgesics for Postsurgical Pain in Rats. Journal of the American Association for Laboratory Animal Science : JAALAS 2015;54:420-5.

17. Sorge RE, Mapplebeck JCS, Rosen S, et al. Different immune cells mediate mechanical pain hypersensitivity in male and female mice. Nat Neurosci 2015;18:1081-3.

18. Sorge RE, LaCroix-Fralish ML, Tuttle AH, et al. Spinal cord Toll-like receptor 4 mediates inflammatory and neuropathic hypersensitivity in male but not female mice. The Journal of neuroscience : the official journal of the Society for Neuroscience 2011;31:15450-4.

19. Wang X, Traub RJ, Murphy AZ. Persistent pain model reveals sex difference in morphine potency. American journal of physiology Regulatory, integrative and comparative physiology 2006;291:R300-6.

20. Loyd DR, Wang X, Murphy AZ. Sex differences in micro-opioid receptor expression in the rat midbrain periaqueductal gray are essential for eliciting sex differences in morphine analgesia. The Journal of neuroscience : the official journal of the Society for Neuroscience 2008;28:14007-17. 
21. Kharwadkar N, Naique S, Molitor PJ. Prospective randomized trial comparing absorbable and nonabsorbable sutures in open carpal tunnel release. Journal of hand surgery (Edinburgh, Scotland) 2005;30:92-5.

22. Sajid MS, Parampalli U, Baig MK, McFall MR. A systematic review on the effectiveness of slowlyabsorbable versus non-absorbable sutures for abdominal fascial closure following laparotomy. International journal of surgery (London, England) 2011;9:615-25. 
Chapter

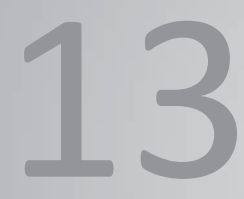

General discussion \& future perspectives 



\section{GENERAL DISCUSSION}

Approximately 3 million abdominal wall hernia repairs are performed annual throughout the world, making hernia repair one of the most frequently performed surgical interventions. Inguinal hernias are by far the most common form of abdominal wall hernia, approximately 75\%, followed by umbilical hernias, epigastric hernias and incisional hernias in decreasing order of incidence. ${ }^{1}$ This thesis mainly focusses on ventral abdominal wall hernias, a great deal of which can be considered a complication of previous surgery as $20 \%$ of all midline laparotomies will end in an incisional hernia within 10 years. ${ }^{2,3}$ After the introduction of laparoscopy, the incidence of incisional hernias was expected to decline, though based on the latest insights this theory seems incorrect, at least for colorectal surgery. ${ }^{4,5}$ During recent years both the incidence and cost for inpatient ventral hernia repair have gone up. ${ }^{6}$

The most significant improvement in the outcome of hernia repair was achieved through the introduction of surgical mesh in the 1940's and 50's that resulted in a large reduction of hernia recurrences. ${ }^{7}$ Though there's no time for celebration yet, as the current recurrence rate ranges from $11 \%$ in the general population to $39 \%$ in complex populations. ${ }^{8-10}$ As a consequence of the widespread use of mesh in hernia repair the consumables industry surrounding hernia repair has expanded drastically, and is expected to grow to $\$ 6.1$ billion by the year $2020 .{ }^{11-14}$ Considering the procedural costs, ranging from approximately $\$ 4.000$ to $\$ 16.000$ for outpatient and inpatient ventral hernia repair respectively, the USA alone spends over $\$ 3.2$ billion on hernia associated healthcare each year. ${ }^{15,16}$ The national costs for hernia repair varies between nations due to differences in surgeon's perspectives, reimbursements, and patient populations. For instance, the US alone holds almost $58 \%$ of the value of the biologic mesh market, indicating a frequent use of these expensive meshes made from either human or animal extracellular matrix. ${ }^{17}$ Given the immense cost of hernia care, a recent publication of Poulose et al. estimates that every $1 \%$ reduction of the recurrence rate would cause a $\$ 32$ million annual saving in the USA alone. ${ }^{16}$

Contrary to the well-developed device industry surrounding hernia repairs, the scientific and clinical field is still developing. Despite the importance of hernia research from a financial and patient perspective, hernia research in general is frequently considered a second-rate problem as it would only concern elective care for a minor nonlife-threatening condition. These assumptions are wrong. Having any type of abdominal wall hernia has a severe impact on patients quality-of-life (QoL) and body image. ${ }^{18-21}$ Fortunately, the majority of hernia repairs are elective, though a national database study from Denmark states that $10 \%$ of the countries hernia repairs are performed as emergency procedures. ${ }^{22,23}$ Other countries, such as the United Kingdom (13\% of all hernias) and the United states (15.6\% of all inpatient hernia repairs) report a comparable incidence of emergency hernia repairs. ${ }^{6,24}$ An alarming statement as the outcome of emergency hernia repair is associated with a fifteenfold higher risk of mortality, reoper- 
ation and readmission compared to elective procedures. ${ }^{10}$ Given the immense impact of hernia repair on the global healthcare industry and on patients QoL, hernia research is of paramount importance for both the patient, and the society. Hernia research can be divided in three equally important categories of management; preoperative, intraoperative, and postoperative.

Despite being in an entirely different field, the financial investment industry requires a work-up similar to ventral hernia management and can serve as an excellent analogy to make the challenges in each category of hernia management more insightful. The first step in any financial investments is balancing the risks and potential gain by using every source of information available to answer one simple question: 'what do I stand to gain from this investment?'. For hernia patients and surgeons the risk-gain balance and hence the central question 'what does the patient stand to gain from this procedure?' is exactly the same. To answer this question accurately, every available source of information must be used to estimate the patients risk for complications accurately and balance the risks versus the potential reward.

\section{PREOPERATIVE}

Risk factors can either be hernia related, procedure/mesh related, or patient related. With the advancement of computer technology, it is possible to build assessment tools that can predict readmission, costs, or complication risk based on many different risk factors. The team of Rosen et al. build the Carolina Equation for Determining Associated Risks (CeDAR) app that can accurately calculate a patient's risk for wound complications based on eight simple questions such as BMI, comorbidity, and type of hernia. ${ }^{25}$ The programme of Baltadano et al. goes even further, combining numerous readily available patient characteristics in an advanced programme that predicts the postoperative readmission risk. ${ }^{26}$ Despite these advances, conventional risk factors such as BMI, smoking, prescription drug use, age, comorbidity etcetera can only take us so far, even if they are combined in advanced software programmes. True innovation lies in the identification of new risk factors. Recently a whole new 'world' of information has become available to us, the olfactory medical evaluation. Every patient exhales thousands of volatile organic compounds with every breath, essentially creating a volatile 'breathprint'. ${ }^{27}$ Measuring and interpreting the exhaled air using an electronic nose (eNose) has opened the door to identify collagen disorders before surgery. ${ }^{28}$ A simple procedure that can be performed at the outpatient clinic within five minutes. This low-cost measurement can improve the risk assessment of patients and possibly change the entire diagnostic workup, as is already happening for lung cancer, rectal cancer, and many other diseases. ${ }^{28,29}$ The results presented in this thesis suggest that the eNose can be used to identify patients with recurrent ventral hernias and aortic aneurysms. Further research is necessary to investigate the true sensitivity and specificity of this approach, though the con- 
cept is intriguing. The eNose has the potential to drastically change the work-up of hernia patients as previous studies have already indicated an altered collagen composition and turnover in patients with incisional hernias or aortic aneurysms. ${ }^{30,31}$ Implementing the eNose in a hernia population could lead to easy identification of vulnerable groups such as Ehlers Danlos patients or the previously mentioned aortic aneurysm patients. Early identification of these vulnerable patients could for instance facilitate the prophylactic mesh placement in aneurysm patients or morbidly obese patients. ${ }^{32-34}$

Alongside with the olfactory diagnostics, imaging modalities can provide valuable evidence in analysing a patient's risk for complications. Currently preoperative CT-scans are being used to evaluate hernia size and location. ${ }^{35-37}$ The standard application of preoperative CT-scans is still heavily debated, though preoperative imaging can help to predict the need for complex hernia repair techniques. ${ }^{38}$ Besides anatomical information the abdominal CT-scan can also provide very accurate hernia size and volume measurements, though these measurements are not routinely performed because of their time consumption and limited clinical evidence. ${ }^{39,40}$ Recent work of Sabbagh et al. suggested a correlation between hernia volume and abdominal compartment syndrome, and Gaidukov et al. showed a correlation between pulmonary intra-abdominal pressure and hernia volume. ${ }^{41,42}$ The results of this thesis show a direct correlation between hernia volume and postoperative pulmonary complications, and opened the door towards more extensive use of imaging modalities in the preoperative work-up of patients. Using the pre-operative abdominal CT-scan the risk of pulmonary complications can be accurately calculated based on the volume of the hernia, and the intraperitoneal volume. This evidence gives merit to the popular theory 'the bigger the hernia, the more complications'. Alongside the previously mentioned CT-based innovations, MRI-scans can provide valuable information as well. Recently the 'dynamic' MRI, or CineMRI, made it possible to reliably evaluate intra-abdominal adhesions. ${ }^{43,44}$ This technique can be used to estimate the duration of adhesiolysis before surgery or evaluate patients with chronic abdominal pain for adhesions related complaints. CineMRI could be a long anticipated non-invasive method of quantifying (mesh-related) intraabdominal adhesions.

\section{INTRAOPERATIVE}

If we stay with the financial investment analogy for just a bit longer, we have now performed the first step of the investment, 'risk assessment'. The next step is to ensure the durability of the investment, as we are in this for the long haul. In hernia surgery, durability is dependent on three things, the previously discussed patient specific risk factors, the quality of the materials used, and the technique associated with their application.

There are only a few certainties in hernia surgery, though the necessity to use mesh in hernias larger than $3 \mathrm{~cm}$ is one of them. ${ }^{45}$ The reduction of recurrence rates through 
the application of surgical mesh is a well-established fact to ensure long-term durability. ${ }^{7,45}$ As previously discussed, the industry surrounding hernia surgeries is immense. Consequently, the market of hernia repair devices is flooded with approximately 200300 currently available meshes. ${ }^{46,47}$ Even the most experienced hernia surgeons will struggle to make an evidence-based decision in this maze of mesh characteristics. Instead of comparing individual meshes, comparing mesh characteristics is a more comprehensive method to assess and compare the clinical performance. Implementing a seal of approval for meshes, as suggested by Deerenberg in her thesis 'Prevention and treatment of incisional hernia', would be an excellent step towards standardisation of non-complex hernias. ${ }^{48}$

Surgical mesh can be categories based on several categories such as: material, weight, pore size, absorbability, and surface modification. In this thesis, two types of commercially available meshes, polypropylene and polyester were compared in a subcutaneous rat model. All meshes resulted in equal tissue ingrowth, though remarkable differences between the seemingly comparable meshes do exist. The polyester mesh hardly shrinks, contrary to the polypropylene meshes, an observation confirmed by multiple studies. ${ }^{49-51}$ Moreover, there are noticeable differences in collagen 1:3 ratios and inflammatory response between polypropylene and polyester mesh, as well as between different types of polypropylene meshes. The collagen 1:3 ratio largely determines the strength of the collagen tissue formed during wound healing or mesh ingrowth. $^{52}$

Mesh ingrowth is not only dependant on the type of mesh used, but also on the location in which the mesh is placed, the overlap and fixation of the mesh, and individual patient characteristics. ${ }^{53,54}$ As mentioned previously, there are absorbable meshes available for ventral hernia repair. An odd development as it seemingly contradicts the previous statement of 'every hernia over $3 \mathrm{~cm}$ needs a mesh'. The absorbable mesh is a step towards 'the ideal' mesh that is only present at the time it is needed and gone by the time it is not. ${ }^{55}$ Ideally, the absorbable mesh will over time be replaced by collagen that will reinforce the abdominal wall and 'take over' the strength of the mesh. Though it remains to be seen if the absorbable mesh leads to higher QoL over time, and whether it has a similar or better recurrence rate than partially or non-absorbable meshes. Previous research has shown that partially absorbable meshes have no clear advantage over lightweight non-absorbable meshes. ${ }^{56}$ Despite potential benefits over time, the absorbable mesh does come with an extra risk, as it is currently not known if patients with altered collagen quality such as Ehlers-Danlos patients, aortic aneurism patients or patients with recurrent hernias have a higher recurrence rate after receiving a fully absorbable mesh compared to a non-absorbable mesh.

In ventral hernia repair the mesh can be placed on top of the rectus abdominis muscles (onlay mesh), underneath the rectus abdominis muscles (sublay mesh), underneath the posterior rectus fascia (Intra-peritoneal onlay mesh), or inside the abdominal cavity (intra-abdominal mesh). Each mesh position has its own pro- and disadvantages. The 
most frequently used mesh position is the sublay position. ${ }^{57}$ Being encapsulated between the rectus abdominis muscles and the posterior rectus fascia, the mesh has a maximum ingrowth surface without direct contact with the viscera, hence minimizing the risk for mesh-related complications, such as fistulas, adhesions, or mesh infection. ${ }^{58,59}$ The sublay position is usually combined with a Rives-Stoppa repair, in which the anterior and posterior rectus fascia are sutured in the midline and the mesh is positioned on top of the posterior rectus fascia. ${ }^{60}$ In some cases it is not possible to close the midline using only the Rives-Stoppa technique, for instance because the posterior rectus fascia cannot reach the midline due to loss of domain. ${ }^{61}$ Loss of domain can occur in patients with large ventral abdominal wall hernias when the lateral abdominal wall muscles retract laterally. ${ }^{62}$ This has created a situation in which the hernia content can't be reduced inside the abdomen because there simply isn't enough room. One solution for this situation is the Endoscopically assisted Components Separation Technique (ECST), described in this thesis. This technique is a modification of the open components separation technique (CST) introduced by Ramirez et al. in 1990. ${ }^{63}$ During the ECST procedure the external oblique muscle is released from its medial insertion allowing the medialisation of the posterior and anterior rectus fascia. ${ }^{64,65}$ The endoscopic modification was developed to reduce the high wound complication rate of the original technique by reducing the wound surface and preserving the innervation and vascularisation of the central abdominal skin. ${ }^{65,66}$ A similar modification of the original CST is the posterior CST, though instead of releasing the external oblique muscle, the lateral edge of the posterior rectus sheath is incised to create a plane between the internal oblique and transverse abdominis muscles. ${ }^{67}$ This procedure can be carried out with or without transection of the transverse abdominis muscle (TAR). Both the ECST and posterior CST, with or without TAR have proven effective in reducing wound complication rates. ${ }^{68,69}$

A different 'go to' repair for hernias that can't be repaired solely using the RivesStoppa technique is the modified Chevrel technique. The modified Chevrel technique is an onlay technique that 'turns over' the anterior rectus fascia to cover the hernia defect and positions an onlay mesh to replace the anterior rectus fascia. The mesh is either positioned on top of the anterior rectus fascia (original technique) or in between the lateral remnants of the anterior rectus fascia in the modified procedure. ${ }^{70,71}$ The technique leads to excellent results regarding quality-of-life and recurrence rates, yet it not frequently used because surgeons fear seroma formation. An understandable, yet unnecessary response as seromas occur in the majority of intra-abdominal procedures though only have clinical repercussions in $2 \%$ of cases. ${ }^{72,73}$ Therefore it seems that not the incidence of seromas, yet the detection of them is increased after onlay mesh placement due to the proximity of the mesh to the skin. Despite taking two hours to perform, the modified Chevrel technique is relatively straightforward and offers a great method for treating complex hernias using a 'simple' technique. Given the excellent quality-of-life, low recurrence rate, and straightforward surgical technique, the modified Chevrel technique deserves a place in modern ventral hernia repair. 
The previously discussed techniques are excellent for hernia repair, yet not every patient that is referred to the hernia outpatient clinic needs surgery. Some may not even have a hernia at all. Given the clinical similarity with hernias, rectus diastasis patients are frequently referred to the hernia surgeon for treatment. This thesis has explored the surgical and physiotherapeutic treatment options for rectus diastasis. Current literature describes roughly four different approaches, three surgical and one physiotherapeutic. Surgical approaches range from hernia repair techniques such as the previously discussed Chevrel and Rives-Stoppa approaches, to tailor made minimally invasive techniques such as the ELAR plus or eMilos repairs and standard plication techniques of the anterior or posterior rectus fascia. ${ }^{74-81}$ Due to the low quality of the included studies, differences between the outcomes of these techniques could not be found. Despite the appeal of treating rectus diastasis with physiotherapy, current literature does not describe the successful treatment of rectus diastasis using physiotherapy. There are some cases in which the inter-rectus distance during muscle contraction could be reduced through physiotherapy exercises, though the impact of this finding for QoL, function, cosmetic result or patient satisfaction is unclear. Moreover, there is great variability in the type of physiotherapy exercises, the duration, and the intensity of the provided programs. Indicating both a necessity for further research in rectus diastasis treatment and a necessity for objective measurement of inter-rectus distance. As published by Water et al. in 2016, inter-rectus distance should be measured using dial callipers or ultrasound. ${ }^{82}$ According to the Beer classification inter-rectus distance must be measured in a relaxed position at $3 \mathrm{~cm}$ above and $2 \mathrm{~cm}$ below the umbilicus. ${ }^{83}$

\section{POSTOPERATIVE}

Referring to the financial investment analogy one last time we have now made an evidence-based investment. The final step is to evaluate the investment and the decision making proces. No matter how good the primary information was, evaluating the outcome is the only appropriate feedback mechanism to improve the preoperative approach and prevent future oversight. ${ }^{84}$

Unfortunately, there are several common complications after hernia surgery, three of which are pain, adhesions, and recurrence. Pain, and in a broader sense QoL, are arguably the most important outcomes of any surgical intervention. If a procedure does not improve the patients current or future QoL, what good can it do?

Despite the importance of QoL, the term is very broad and hence it is difficult to quantify. There are generic QoL questionnaires such as the SF-36 or EQ-5D, though they may not be as sensitive to the impact of surgical interventions due to their non-specific setup. ${ }^{85,86}$ Disease specific QoL questionnaire, such as the COMI-hernia, can solve this problem. ${ }^{87}$ A disease specific QoL questionnaire asks Patient Reported Outcomes (PROs) relevant for a specific disease. This thesis describes the first clinical application of the 
COMI-hernia questionnaire, besides the original validation paper. ${ }^{87,88}$ Overall, the COMIhernia questionnaire worked well and succeeded in defining a set of core outcome criteria for QoL in a hernia population, though the interpretation of the questionnaire is quite difficult. Ever since the introduction of the COMI-hernia questionnaire, the European Registry of Abdominal Wall Hernias (EuraHS) has developed a similar hernia specific QoL questionnaire, the EuraHS-QoL. ${ }^{89}$ This questionnaire focusses on a similar set of core outcomes with the addition of cosmesis. Moreover, it can be used both pre- and postoperative for any type of hernia. Clinical validation of the EuraHS questionnaire for inguinal hernias is completed and validation for ventral abdominal wall hernias is ongoing. ${ }^{90}$ The EuraHS-QoL is available for free in many different languages and can be seamlessly incorporated in the world-wide EuraHS online registration database. ${ }^{89}$

Postoperative adhesion formation following abdominal wall repair with an intraperitoneally placed mesh remains an underestimated and difficult problem. ${ }^{91,92}$ Adhesions are fibrous connections between organs or tissues that were previously unconnected, frequently seen in response to trauma or inflammation of the involved tissues. ${ }^{93-95}$ Despite numerous attempts of adhesion reduction through the applications of drugs and barriers, adhesions still occur after nearly all intra-abdominal procedures. ${ }^{96}$ The high incidence of adhesions has created a flood of anti-adhesive products that have mediocre results at best. ${ }^{97,98}$ Evaluating the outcome of anti-adhesive materials in an experimental setting is becoming increasingly difficult due to the multitude of different adhesion scores. There are currently so many adhesion scores available that the interstudy comparability is low. To increase the interstudy comparability a DELPHI analysis concerning mesh related adhesion scoring was performed, using the input of an international panel of experts in adhesions research. Based on the outcomes of the DELPHI analysis, the 2016 EHS adhesion consensus score* was developed. Instead of adding 'just another score' on the pile of adhesion scoring methods, the EHS 2016 adhesion consensus score can be used as a minimal set of outcomes, paving the way toward comparable outcome reporting in mesh related adhesion research. Alongside the development of the EHS 2016 adhesion consensus score, the DELPHI analysis resulted in many recommendations towards mesh related adhesions research, including follow-up length, outcome measurement, clinical significance and many more.

Overall this thesis has attempted to evaluate, develop, and reflect on advances concerning all three domains of abdominal wall hernia management. Only time will tell if it has succeeded. It was an honour and a privilege to participate in the academic world of hernia repair.

*name approval by EHS pending 


\section{FUTURE PERSPECTIVES}

If mesh development continuous on its current pace, several years from now there will be five-hundred different meshes to choose from and evidence based mesh comparisons will become impossible the fate of anti-adhesive materials will be quite grim, as the current 'trial and error' method to develop and evaluate anti-adhesive materials during the past decades has only resulted in two moderately successful anti-adhesive materials. ${ }^{99-103}$ To create a more positive future for hernia and adhesion research altogether, we must come to terms with the following two facts. The first is, we have enough meshes. The second is, we need to stop trying to prevent adhesions until we know the pathophysiology. Accepting this will enable funding of fundamental research focussing on the following three topics.

\section{Evaluation \& standardisation}

It seems that we are so eager to 'develop' that we no longer evaluate current practice. Nor do we implement the knowledge that is already available. Lack in standardisation has created the shocking outcome of the 2013 study of Funk et al. stating that laparoscopy is being underused in the US for hernia repair, and $70 \%$ of primary hernias are repaired without mesh, despite overwhelming evidence regarding the efficacy of mesh in reducing recurrences. ${ }^{104}$

The current level of standardisation is insufficient. Standardisation should go as far as stating the type and size of mesh to be used for different types of ventral hernias and stating the requirements for 'hernia' surgeons to operate on complex cases. The fundamentals are already published by the European Hernia Society (EHS) in their classification tool, and by Slater et al. in his publication on complex hernias. ${ }^{105}$ The noncomplex hernia repairs need standardisation, as mentioned previously. Some attempts to standardise non-complex hernia repair have been published, though consensusbased guideline from the European Hernia Society are unavailable. ${ }^{106-108}$ Contrary to the non-complex hernias, complex hernias require a tailored approach, though the patient work-up can be standardised. To facilitate surgeons in their mesh choice and prevent flooding of the market, a certificate of approval could be administered by the EHS or World Hernia Society (WHS), approving the mesh for one or several types of repair.

\section{Fundamental pathophysiology research \& prevention}

The pathophysiology of ventral hernias, either incisional of primary, has not been unraveled to date, nor has the pathophysiology of adhesions. This deficit in the evidencebased approach towards ventral hernia repair and adhesion prevention is not due to lack of interest or technological means. It is primarily due to a lack of funding. The technique for detailed research in the pathophysiology of hernia formation and adhesion 
formation has been available for years. Using Mass Spectrometry Imaging (MSI) we can image just about anything with a mass in high spatial resolution. ${ }^{109}$ Though the main drawback from this technology is the price.

Without spending a single dollar, we can start with prevention of hernia formation today. Multiple studies on high risk populations for hernia formation, such as aortic aneurysm patients, indicate that the use of prophylactic mesh placement is safe and cost-efficient, not to mention desirable for the patient. ${ }^{32-34,110}$ Implementing the 4:1 suture to wound length ratio and preventive mesh placement would immensely reduce the incidence of incisional hernias throughout the world.

\section{Patient reported outcome measurement}

For centuries, medical research has focused on outcomes that are completely irrelevant for the patient. What good is adhesion reduction if there is no clinical implication? And what good is an absorbable mesh if it doesn't improve quality of life? The patient doesn't care if the doctor is happy with the result, but the doctor should care about patient satisfaction. Ultimately, medical research should impact the patient and we should choose clinical outcomes accordingly. Patient reported outcomes should be plentiful and kept in high regard, though currently they are scarce and hardly published in high impact journals. ${ }^{111}$

Focussing on these three domains for improvement of hernia care will result in a more standardised practice and will eventually increase the overall quality of hernia care and research. 


\section{REFERENCES}

1. Dabbas N, Adams K, Pearson K, Royle GT. Frequency of abdominal wall hernias: is classical teaching out of date? JRSM Short Reports 2011;2:5.

2. Fink $C$, Baumann $P$, Wente $M N$, et al. Incisional hernia rate 3 years after midline laparotomy. The British journal of surgery 2014;101:51-4.

3. Mudge M, Hughes LE. Incisional hernia: a 10 year prospective study of incidence and attitudes. The British journal of surgery 1985;72:70-1.

4. Mishra A, Keeler BD, Maxwell-Armstrong C, Simpson JA, Acheson AG. The influence of laparoscopy on incisional hernia rates: a retrospective analysis of 1057 colorectal cancer resections. Colorectal disease : the official journal of the Association of Coloproctology of Great Britain and Ireland 2014;16:815-21.

5. Kuhry E, Schwenk WF, Gaupset R, Romild U, Bonjer HJ. Long-term results of laparoscopic colorectal cancer resection. The Cochrane database of systematic reviews 2008:Cd003432.

6. Beadles CA, Meagher AD, Charles AG. Trends in emergent hernia repair in the United States. JAMA surgery 2015;150:194-200.

7. Luijendijk RW, Hop WC, van den Tol MP, et al. A comparison of suture repair with mesh repair for incisional hernia. The New England journal of medicine 2000;343:392-8.

8. Liang MK, Berger RL, Li LT, Davila JA, Hicks SC, Kao LS. Outcomes of laparoscopic vs open repair of primary ventral hernias. JAMA surgery 2013;148:1043-8.

9. Slater NJ, van Goor H, Bleichrodt RP. Large and complex ventral hernia repair using "components separation technique" without mesh results in a high recurrence rate. Am J Surg 2015;209:170-9.

10. Helgstrand F. National results after ventral hernia repair. Danish medical journal 2016;63.

11. Koontz AR. Preliminary report on the use of tantalum mesh in the repair of ventral hernias. Transactions of the Southern Surgical Association Southern Surgical Association (US) 1947;59 (1 vol.):382-8.

12. Koontz AR. Tantalum gauze in hernia repair. Sinai Hospital journal 1952;1:13-20.

13. Aghina A. [Use of nylon mesh in the surgical treatment of laparoceles and hernia]. Giornale italiano di chirurgia 1951;7:508-10.

14. Dhabale S. Hernia Repair Devices and Consumables Market by Hernia Type (Incisional, inguinal, femoral, Umbilical), Surgery Type (Open tension-free repair, Laparoscopic surgery), Product Type (Devices, Consumables, Synthetic material, Absorbable, Biological Material) - Global Opportunity Analysis and Industry Forecast, 2013 - 2020: Allied market research; 2015.

15. Vorst AL, Kaoutzanis C, Carbonell AM, Franz MG. Evolution and advances in laparoscopic ventral and incisional hernia repair. World Journal of Gastrointestinal Surgery 2015;7:293-305.

16. Poulose BK, Shelton J, Phillips S, et al. Epidemiology and cost of ventral hernia repair: making the case for hernia research. Hernia 2012;16:179-83.

17. Data Bridge Market Research. Global Hernia Mesh Repair Devices Market - Trends and Forecast to 20242015.

18. Rogmark P, Petersson U, Bringman S, Ezra E, Osterberg J, Montgomery A. Quality of Life and Surgical Outcome 1 Year After Open and Laparoscopic Incisional Hernia Repair: PROLOVE: A Randomized Controlled Trial. Annals of surgery 2016;263:244-50.

19. van Dijk SM, Timmermans L, Deerenberg EB, et al. Parastomal Hernia: Impact on Quality of Life? World journal of surgery 2015;39:2595-601.

20. Palmqvist E, Larsson K, Anell A, Hjalmarsson C. Prospective study of pain, quality of life and the economic impact of open inguinal hernia repair. The British journal of surgery 2013;100:1483-8.

21. van Ramshorst GH, Eker HH, Hop WC, Jeekel J, Lange JF. Impact of incisional hernia on health-related quality of life and body image: a prospective cohort study. Am J Surg 2012;204:144-50.

22. Helgstrand F, Rosenberg J, Bay-Nielsen M, et al. Establishment and initial experiences from the Danish Ventral Hernia Database. Hernia 2010;14:131-5.

23. Israelsson LA, Smedberg S, Montgomery A, Nordin P, Spangen L. Incisional hernia repair in Sweden 2002. Hernia 2006;10:258-61. 
24. Andrews NJ. Presentation and outcome of strangulated external hernia in a district general hospital. The British journal of surgery 1981;68:329-32.

25. Carolinas Surgical Innovation Group. The Carolinas Equation for Determining Associated Risks (CeDAR). Carolina HealthCare system; 2014.

26. Baltodano PA, Webb-Vargas $Y$, Soares KC, et al. A validated, risk assessment tool for predicting readmission after open ventral hernia repair. Hernia 2016;20:119-29.

27. Amann A, Costello Bde L, Miekisch W, et al. The human volatilome: volatile organic compounds (VOCs) in exhaled breath, skin emanations, urine, feces and saliva. Journal of breath research 2014;8:034001.

28. Nakhleh MK, Amal H, Jeries R, et al. Diagnosis and Classification of 17 Diseases from 1404 Subjects via Pattern Analysis of Exhaled Molecules. ACS Nano 2017;11:112-25.

29. Bikov A, Lazar Z, Horvath I. Established methodological issues in electronic nose research: how far are we from using these instruments in clinical settings of breath analysis? Journal of breath research 2015;9:034001.

30. Henriksen NA, Mortensen JH, Sorensen LT, et al. The collagen turnover profile is altered in patients with inguinal and incisional hernia. Surgery 2015;157:312-21.

31. Rodella LF, Rezzani R, Bonomini F, et al. Abdominal aortic aneurysm and histological, clinical, radiological correlation. Acta histochemica 2016;118:256-62.

32. Muysoms FE, Detry O, Vierendeels T, et al. Prevention of Incisional Hernias by Prophylactic Meshaugmented Reinforcement of Midline Laparotomies for Abdominal Aortic Aneurysm Treatment: A Randomized Controlled Trial. Annals of surgery 2016;263:638-45.

33. Muysoms FE, Dietz UA. Prophylactic meshes in the abdominal wall. Der Chirurg; Zeitschrift fur alle Gebiete der operativen Medizen 2017;88:34-41.

34. Timmermans L, Eker HH, Steyerberg EW, et al. Short-term results of a randomized controlled trial comparing primary suture with primary glued mesh augmentation to prevent incisional hernia. Annals of surgery 2015;261:276-81

35. Yao S, Li JY, Liu FD, Pei LJ. Significance of measurements of herniary area and volume and abdominal cavity volume in the treatment of incisional hernia: application of CT 3D reconstruction in 17 cases. Comput Aided Surg 2012;17:40-5.

36. Tanaka EY, Yoo JH, Rodrigues AJ, Jr., Utiyama EM, Birolini D, Rasslan S. A computerized tomography scan method for calculating the hernia sac and abdominal cavity volume in complex large incisional hernia with loss of domain. Hernia 2010;14:63-9.

37. Agnew SP, Small W, Jr., Wang E, Smith LJ, Hadad I, Dumanian GA. Prospective measurements of intraabdominal volume and pulmonary function after repair of massive ventral hernias with the components separation technique. Annals of surgery 2010;251:981-8.

38. Blair LJ, Ross SW, Huntington CR, et al. Computed tomographic measurements predict component separation in ventral hernia repair. The Journal of surgical research 2015;199:420-7.

39. Tanaka EY, Yoo JH, Rodrigues AJ, Utiyama EM, Birolini D, Rasslan S. A computerized tomography scan method for calculating the hernia sac and abdominal cavity volume in complex large incisional hernia with loss of domain. Hernia 2010;14:63-9.

40. Yao S, Li JY, Liu FD, Pei LJ. Significance of measurements of herniary area and volume and abdominal cavity volume in the treatment of incisional hernia: application of CT 3D reconstruction in 17 cases. Comput Aided Surg 2012;17:40-5.

41. Gaidukov KM, Raibuzhis EN, Hussain A, et al. Effect of intra-abdominal pressure on respiratory function in patients undergoing ventral hernia repair. World J Crit Care Med 2013;2:9-16.

42. Sabbagh C, Dumont F, Robert B, Badaoui R, Verhaeghe P, Regimbeau JM. Peritoneal volume is predictive of tension-free fascia closure of large incisional hernias with loss of domain: a prospective study. Hernia : the journal of hernias and abdominal wall surgery 2011;15:559-65.

43. Randall D, Fenner J, Gillott R, et al. A Novel Diagnostic Aid for Detection of Intra-Abdominal Adhesions to the Anterior Abdominal Wall Using Dynamic Magnetic Resonance Imaging. Gastroenterology research and practice $2016 ; 2016: 2523768$. 
44. Lang RA, Buhmann S, Hopman A, et al. Cine-MRI detection of intraabdominal adhesions: correlation with intraoperative findings in 89 consecutive cases. Surgical endoscopy 2008;22:2455-61.

45. Silecchia G, Campanile FC, Sanchez L, et al. Laparoscopic ventral/incisional hernia repair: updated Consensus Development Conference based guidelines [corrected]. Surgical endoscopy 2015;29:2463-84.

46. Klinge $U$, Klosterhalfen $B$. Modified classification of surgical meshes for hernia repair based on the analyses of 1,000 explanted meshes. Hernia : the journal of hernias and abdominal wall surgery 2012;16:251-8.

47. Klinge U, Park JK, Klosterhalfen B. 'The ideal mesh?'. Pathobiology : journal of immunopathology, molecular and cellular biology 2013;80:169-75.

48. Deerenberg EB. Prevention and Treatment of Incisional Hernia: New Techniques and Materials: Erasmus universiteit Rotterdam; 2017.

49. Brown CN, Finch JG. Which mesh for hernia repair? Annals of The Royal College of Surgeons of England 2010;92:272-8.

50. Klinge U, Klosterhalfen B, Müller M, Öttinger AP, Schumpelick V. Shrinking of Polypropylene Mesh in vivo: An Experimental Study in Dogs. The European Journal of Surgery 1998;164:965-9.

51. Coda A, Bendavid R, Botto-Micca F, Bossotti M, Bona A. Structural alterations of prosthetic meshes in humans. Hernia : the journal of hernias and abdominal wall surgery 2003;7:29-34.

52. Junge $K$, Klinge $U$, Rosch $R$, et al. Decreased collagen type $I / I I I$ ratio in patients with recurring hernia after implantation of alloplastic prostheses. Langenbeck's archives of surgery 2004;389:17-22.

53. Binnebosel M, Klink CD, Otto J, et al. Impact of mesh positioning on foreign body reaction and collagenous ingrowth in a rabbit model of open incisional hernia repair. Hernia : the journal of hernias and abdominal wall surgery 2010;14:71-7.

54. Grotenhuis N, Vd Toom HF, Kops N, et al. In vitro model to study the biomaterial-dependent reaction of macrophages in an inflammatory environment. The British journal of surgery 2014;101:983-92.

55. Scott JR, Deeken CR, Martindale RG, Rosen MJ. Evaluation of a fully absorbable poly-4hydroxybutyrate/absorbable barrier composite mesh in a porcine model of ventral hernia repair. Surgical endoscopy 2016;30:3691-701.

56. Rickert A, Kienle P, Kuthe A, et al. A randomised, multi-centre, prospective, observer and patient blind study to evaluate a non-absorbable polypropylene mesh vs. a partly absorbable mesh in incisional hernia repair. Langenbeck's archives of surgery 2012;397:1225-34.

57. Timmermans L, de Goede B, van Dijk SM, Kleinrensink GJ, Jeekel J, Lange JF. Meta-analysis of sublay versus onlay mesh repair in incisional hernia surgery. Am J Surg 2014;207:980-8.

58. Holihan JL, Bondre I, Askenasy EP, et al. Sublay versus underlay in open ventral hernia repair. The Journal of surgical research 2016;202:26-32.

59. Timmermans L, de Goede B, van Dijk SM, Kleinrensink GJ, Jeekel J, Lange JF. Meta-analysis of sublay versus onlay mesh repair in incisional hernia surgery. Am J Surg 2014;207:980-8.

60. Stoppa RE, Warlaumont CR, Verhaeghe PJ, Romero ER, M'Balla-N'Di CJ. Prosthetic repair in the treatment of groin hernias. International surgery 1986;71:154-8.

61. Renard Y, Lardiere-Deguelte S, de Mestier L, et al. Management of large incisional hernias with loss of domain: A prospective series of patients prepared by progressive preoperative pneumoperitoneum. Surgery 2016;160:426-35.

62. Alyami M, Passot G, Voiglio E, et al. Feasibility of Catheter Placement Under Ultrasound Guidance for Progressive Preoperative Pneumoperitoneum for Large Incisional Hernia with Loss of Domain. World journal of surgery 2015;39:2878-84.

63. Ramirez OM, Ruas E, Dellon AL. "Components separation" method for closure of abdominal-wall defects: an anatomic and clinical study. Plast Reconstr Surg 1990;86:519-26.

64. Mommers EH, Wegdam JA, Nienhuijs SW, de Vries Reilingh TS. How to perform the endoscopically assisted components separation technique (ECST) for large ventral hernia repair. Hernia : the journal of hernias and abdominal wall surgery 2016;20:441-7.

65. de Vries Reilingh TS, van Goor H, Rosman C, et al. "Components separation technique" for the repair of large abdominal wall hernias. J Am Coll Surg 2003;196:32-7. 
66. Switzer NJ, Dykstra MA, Gill RS, et al. Endoscopic versus open component separation: systematic review and meta-analysis. Surgical endoscopy 2015;29:787-95.

67. Rosen MJ. Atlas of abdominal wall reconstruction. 1st ed: Elsevier Inc.; 2012.

68. Carbonell AM, Cobb WS, Chen SM. Posterior components separation during retromuscular hernia repair. Hernia 2008;12:359-62.

69. Novitsky YW, Elliott HL, Orenstein SB, Rosen MJ. Transversus abdominis muscle release: a novel approach to posterior component separation during complex abdominal wall reconstruction. The American Journal of Surgery;204:709-16.

70. Chevrel JP. Traitement des grandes éventrations médianes par plastie en paletot et prothèse. La Nouvelle Presse Médicale 1979;8:695-6.

71. Chevrel JP, Morquette H. Traitement des éventrations abdominales médianes par autoplastie musculaire et prothèse pré-musculo-aponévrotique. Chirurgie (Mémoires de l'Académie) 1986;9:7.

72. Susmallian S, Gewurtz G, Ezri T, Charuzi I. Seroma after laparoscopic repair of hernia with PTFE patch: is it really a complication? Hernia 2001;5:139-41.

73. Morales-Conde S, Suarez-Artacho G, Socas-Macias M, Barranco-Moreno A. Retroprosthetic seroma after laparoscopic ventral hernia repair: incidence, risk factors and clinical significance. Hernia 2015;19:943-7.

74. Köckerling F, Botsinis MD, Rohde C, Reinpold W. Endoscopic-Assisted Linea Alba Reconstruction plus Mesh Augmentation for Treatment of Umbilical and/or Epigastric Hernias and Rectus Abdominis Diastasis - Early Results. Frontiers in surgery 2016;3:27.

75. Reinpold. Endoscopic assisted mini-open transhernial sublay repair (eMILOS) of symptomatic diastasis recti. Hernia 2016;20.

76. Palanivelu C, Rangarajan M, Jategaonkar PA, Amar V, Gokul KS, Srikanth B. Laparoscopic repair of diastasis recti using the 'Venetian blinds' technique of plication with prosthetic reinforcement: a retrospective study. Hernia : the journal of hernias and abdominal wall surgery 2009;13:287-92.

77. Shirah BHS, H.A. The Effectiveness of Polypropylene Mesh in the Open and Laparoscopic Repair of Divarication of the Recti. Med Imp Surg 2016; Volume 1.

78. Deriugina MS. [A method of surgical treatment of diastasis recti abdominis]. Vestnik khirurgii imeni I I Grekova 2001;160:77-8.

79. Angio LG, Piazzese E, Pacile $V$, et al. [The surgical treatment of the diastasis recti abdominis: an original technique of prosthesis repair of the abdominal wall]. II Giornale di chirurgia 2007;28:187-98.

80. Gireev GI, Zagirov UZ, Shakhnazarov AM. [Treatment of linea alba hernia and diastasis of rectus abdominis]. Khirurgiia 1997:58-61.

81. Kockerling F, Botsinis MD, Rohde C, Reinpold W, Schug-Pass C. Endoscopic-assisted linea alba reconstruction: New technique for treatment of symptomatic umbilical, trocar, and/or epigastric hernias with concomitant rectus abdominis diastasis. European surgery : ACA : Acta chirurgica Austriaca 2017;49:71-5.

82. van de Water AT, Benjamin DR. Measurement methods to assess diastasis of the rectus abdominis muscle (DRAM): A systematic review of their measurement properties and meta-analytic reliability generalisation. Manual therapy 2016;21:41-53.

83. Beer GM, Schuster A, Seifert B, Manestar M, Mihic-Probst D, Weber SA. The normal width of the linea alba in nulliparous women. Clinical anatomy (New York, NY) 2009;22:706-11.

84. Taylor JB. The financial crisis and the policy responses: an empirical analysis of what went wrong: National bureau of economic research; 2009.

85. Rabin R, de Charro F. EQ-5D: a measure of health status from the EuroQol Group. Annals of medicine 2001;33:337-43.

86. Ware JE, Jr., Sherbourne CD. The MOS 36-item short-form health survey (SF-36). I. Conceptual framework and item selection. Medical care 1992;30:473-83.

87. Staerkle RF, Villiger P. Simple questionnaire for assessing core outcomes in inguinal hernia repair. The British journal of surgery 2011;98:148-55

88. Mommers EHH, Hunen DRM, van Hout J, et al. Patient-reported outcomes (PROs) after total extraperitoneal hernia repair (TEP). Hernia 2017;21:45-50. 
89. Muysoms F, Campanelli G, Champault GG, et al. EuraHS: the development of an international online platform for registration and outcome measurement of ventral abdominal wall hernia repair. Hernia 2012;16:239-50.

90. Muysoms FE, Vanlander A, Ceulemans R, et al. A prospective, multicenter, observational study on quality of life after laparoscopic inguinal hernia repair with ProGrip laparoscopic, self-fixating mesh according to the European Registry for Abdominal Wall Hernias Quality of Life Instrument. Surgery 2016;160:1344-57.

91. Schreinemacher MH, ten Broek RP, Bakkum EA, van Goor H, Bouvy ND. Adhesion awareness: a national survey of surgeons. World journal of surgery 2010;34:2805-12.

92. Leclercq RM, Van Barneveld KW, Schreinemacher $\mathrm{MH}$, et al. Postoperative abdominal adhesions and bowel obstruction. A survey among Dutch general practitioners. The European journal of general practice 2015;21:176-82.

93. Wallwiener M, Wallwiener CW, Molinas R, et al. Intraabdominal adhesion formation is associated with differential mRNA expression of metabolic genes PDHb and SDHa. Archives of gynecology and obstetrics 2012;286:683-6.

94. Zuhlke HV, Lorenz EM, Straub EM, Savvas V. [Pathophysiology and classification of adhesions]. Langenbecks Archiv fur Chirurgie Supplement II, Verhandlungen der Deutschen Gesellschaft fur Chirurgie Deutsche Gesellschaft fur Chirurgie Kongress 1990:1009-16.

95. Arung W, Meurisse M, Detry O. Pathophysiology and prevention of postoperative peritoneal adhesions. World J Gastroenterol 2011;17:4545-53.

96. Ouaïssi M, Gaujoux S, Veyrie N, et al. Post-operative adhesions after digestive surgery: Their incidence and prevention: Review of the literature. Journal of Visceral Surgery 2012;149:e104-e14.

97. Menzies D, Pascual MH, Walz MK, et al. Use of icodextrin $4 \%$ solution in the prevention of adhesion formation following general surgery: from the multicentre ARIEL Registry. Ann R Coll Surg Engl 2006;88:375-82.

98. Diamond MP. Reduction of postoperative adhesion development. Fertility and sterility 2016;106:9947.e1.

99. Ahmad G, O'Flynn H, Hindocha A, Watson A. Barrier agents for adhesion prevention after gynaecological surgery. The Cochrane database of systematic reviews 2015:Cd000475.

100. Hindocha A, Beere L, Dias S, Watson A, Ahmad G. Adhesion prevention agents for gynaecological surgery: an overview of Cochrane reviews. The Cochrane database of systematic reviews 2015;1:Cd011254.

101. Trew G, Pistofidis G, Pados G, et al. Gynaecological endoscopic evaluation of $4 \%$ icodextrin solution: a European, multicentre, double-blind, randomized study of the efficacy and safety in the reduction of de novo adhesions after laparoscopic gynaecological surgery. Hum Reprod 2011;26:2015-27. doi: 10.1093/humrep/der135. Epub 2011 Jun 1.

102. Rajab TK, Wallwiener M, Planck C, Brochhausen C, Kraemer B, Wallwiener CW. A direct comparison of seprafilm, adept, intercoat, and spraygel for adhesion prophylaxis. J Surg Res 2010;161:246-9. doi: 10.1016/j.jss.2008.11.839. Epub Dec 10.

103. ten Broek RP, Stommel MW, Strik C, van Laarhoven CJ, Keus F, van Goor H. Benefits and harms of adhesion barriers for abdominal surgery: a systematic review and meta-analysis. Lancet 2014;383:48-59.

104. Funk LM, Perry KA, Narula VK, Mikami DJ, Melvin WS. Current national practice patterns for inpatient management of ventral abdominal wall hernia in the United States. Surgical endoscopy 2013;27:4104-12.

105. Slater NJ, Montgomery A, Berrevoet F, et al. Criteria for definition of a complex abdominal wall hernia. Hernia : the journal of hernias and abdominal wall surgery 2014;18:7-17.

106. Bittner R, Bingener-Casey J, Dietz U, et al. Guidelines for laparoscopic treatment of ventral and incisional abdominal wall hernias (International Endohernia Society [IEHS])-Part III. Surgical endoscopy 2014;28:380-404.

107. Bittner R, Bingener-Casey J, Dietz U, et al. Guidelines for laparoscopic treatment of ventral and incisional abdominal wall hernias (International Endohernia Society (IEHS) - Part 1. Surgical endoscopy 2014;28:2-29.

108. Bittner R, Bingener-Casey J, Dietz U, et al. Guidelines for laparoscopic treatment of ventral and incisional abdominal wall hernias (International Endohernia Society [IEHS])-Part 2. Surgical endoscopy 2014;28:353-79. 
109. Brown VL, Liu Q, He L. Matrix-enhanced surface-assisted laser desorption/ionization mass spectrometry (ME-SALDI-MS) for mass spectrometry imaging of small molecules. Methods in molecular biology (Clifton, NJ) 2015;1203:175-84.

110. Nieuwenhuizen J, Eker HH, Timmermans L, et al. A double blind randomized controlled trial comparing primary suture closure with mesh augmented closure to reduce incisional hernia incidence. BMC surgery 2013;13:48.

111. Antonescu I, Mueller CL, Fried GM, Vassiliou MC, Mayo NE, Feldman LS. Outcomes reported in highimpact surgical journals. The British journal of surgery 2014;101:582-9. 

Chapter

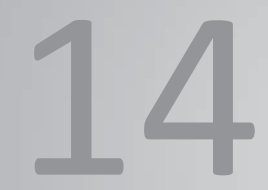

Summary

Summary (Dutch)

Acknowledgements (Dutch)

List of publications

Curriculum Vitae (Dutch)

Valorisation 



\section{Summary}

The goal of this thesis was to evaluate and initiate developments in the field of abdominal wall hernia repair to improve the process of patient selection, surgical reconstruction techniques, postoperative evaluation and scientific research. The chapters of this thesis are structured in preoperative, intraoperative, and postoperative depending on the hernia management phase each chapter relates to.

\section{PREOPERATIVE}

Chapter 2 Describes the application of exhaled breath analysis in a population of recurrent hernia patients and aortic aneurysm patients. Both populations have alterations in their collagen type I:III ratio. Using an electronic nose to analyse the volatile organic compound composition in both populations it was possible to distinguish both recurrent hernia patients and aortic aneurysm patients from healthy controls. This proof of concept study opens the door to selecting preoperative patients for prophylactic mesh placement to prevent incisional hernia formation.

Chapter 3 Analyses the relationship between hernia volume and postoperative pulmonary complications in a retrospective cohort of 35 complex abdominal wall patients. Hernia volume was measured accurately on preoperative abdominal CT-scans with 3D reconstruction techniques. A statistically significant relationship between hernia volume and postoperative pulmonary complications was found, giving merit to the popular theory 'the bigger the hernia, the more complications'.

\section{INTRAOPERATIVE}

Chapter 4 Describes a modified endoscopic operation technique for large ventral abdominal wall defects, the Endoscopically assisted Component Separation Technique (ECST). This technique is a modified version of the Ramirez technique, described in 1990. Using this technique, the wound surface is reduced and the blood supply to the ventral abdominal wall is preserved to reduce the number of wound complications. The technique is performed in 27 patients, analysed prospectively. The results are promising and suggest a better performance of the modified technique compared to the original technique regarding complications, though the width gained by of the endoscopic release is less compared to the original 'open' release of the external oblique muscle. 
Chapter 5 Describes a modified version the Chevrel technique introduced in the 1970's. The relatively easy procedure can be used to treat complex ventral abdominal wall hernias using straightforward technique. This chapter describes the technique in detail and displays the results of the technique in a retrospective, single surgeon cohort of 155 patients. The modified Chevrel technique leads to a high quality of life and is characterised by a low number of recurrences. There is a higher $(23.2 \%)$ complication rate, though the majority of these are seromas. Due to the superficial (onlay) placement of the mesh, seromas are more easily detected compared to sublay or intra-abdominal mesh placement techniques.

Chapter 6 Describes a comparative animal study including four commercially available synthetic non-absorbable meshes, implanted in male Whistar Han rats. The goal is to identify differences in clinical performance of these seemingly comparable meshes. No differences regarding mesh ingrowth were discovered, though after 90 days polypropylene meshes can shrink up to $10 \%$ when compared to baseline.

Chapter $7 \mathrm{~A}$ case is presented of a patient receiving a new type of synthetic absorbable mesh. Several days after surgery the patient presents with large seroma on top of the mesh, with clinical and radiological features of an infected seroma. Infected seromas on synthetic material frequently lead to infection of the material. Against the odds, the patient made a complete recovery after receiving intravenous and oral antibiotics therapy. Analysing this case there are two possible explanations for the positive outcome, either the seroma was not infected, or the mesh has a high resistance to bacterial colonisation.

Chapter 8 Rectus diastasis is a common problem in the hernia outpatient clinic. Despite not being a true hernia but rather a widening of the midline and laxity of the ventral abdominal wall, patients often seek surgical intervention or advice. A systematic literature review is performed to analyse all surgical and physiotherapeutic treatment options for rectus diastasis in terms of patient satisfaction, complication rate and recurrence rate. The analysis shows that complete resolution of rectus diastasis through physiotherapeutic treatment has not been described. Surgical interventions often concern plication techniques, if patients present with concomitant midline hernias the entire midline is reinforced and rectus diastasis is often treated as if it were a hernia. The chapter ends with recommendations and considerations for treatment of rectus diastasis.

\section{POSTOPERATIVE}

Chapter 9 concerns the measurement of Patient Reported Outcome Measures (PROMs) through a new type of disease specific quality of life questionnaire, the COMI-Hernia 
questionnaire, in a prospective cohort of 120 groin hernia patients before and after total extraperitoneal hernioplasty (TEP). This chapter provides the first clinical measurement of the COMI-Hernia questionnaire other than the validation paper of Stearkle et al. Compared to previous publication on quality of life after TEP, a higher percentage of postoperative pain was observed. The COMI-Hernia questionnaire works well in clinical practice, though interpreting the results can be difficult due to lack of experience and/or similarities in several outcome parameters.

Chapter 10 Describes the application of the Delphi technique to reach international consensus on scoring mesh related postoperative adhesions. Based on the outcome of the Delphi analysis a new type of adhesion scoring tool is developed and recommendations on outcome reporting in experimental mesh adhesions research are made.

Chapter 11 Describes a retrospective single center cohort of Ehlers Danlos patients. This group of patients had a disturbed collagen synthesis, potentially predisposing them for both primary and recurrent ventral hernias. The presented cohort includes fourteen patients with different types of Ehlers Danlos, that underwent ventral abdominal wall reconstruction with an extra-large mesh, to compensate for the impaired collagen strength. Remarkably, a recurrence rate of only $7 \%$ was observed after a median followup of 5 years, compared to approximately $12 \%$ in the general population.

Chapter 12 concerns the baseline performance of the ischemic button model, used for the induction of experimental adhesions in male and female Whistar Han rats. The study shows that there is no sex-based difference in baseline performance of the ischemic button model. Male rats show a small but significantly faster postoperative increase in body weight. Though there is no difference in welfare scores or additional analgesia. The faster weight gain in male rats can be explained by a dimorphism in both pain perception as well as pain treatment using opioids. 



\section{Summary (Dutch)}

Het doel van deze thesis was om ontwikkelingen binnen de buikwandchirurgie te evalueren en te initiëren binnen de gebieden van patiëntselectie, chirurgische techniek, evaluatie van postoperatieve resultaten en methoden voor wetenschappelijk onderzoek. De hoofdstukken in deze thesis zijn gestructureerd in preoperatief, intra-operatief en postoperatief om het deelgebied van hernia management waarop zij betrekking hebben inzichtelijk te maken.

\section{PREOPERATIEF}

Hoofdstuk 2 Betreft de eerste studie die kijkt of het mogelijk is om patiënt met een hoog risico op recidief van de buikwandbreuk in een zeer vroeg stadium te herkennen middels analyse van uitgeademde lucht. Dezelfde techniek wordt toegepast in een populatie van aneurysma patiënten omdat beide groepen een afwijkende collageen productie hebben. Deze studie toont aan dat zowel patiënten met een aneurysma van de aorta als patiënten met een recidief hernia door middel van ademanalyse onderscheiden kunnen worden van gezonde controle patiënten. Indien dit concept kan worden vertaald naar de preoperatieve periode kan de eNose mogelijk gebruikt worden als screeningsinstrument voor profylactische mesh plaatsing.

Hoofdstuk 3 Beschrijft een retrospectief onderzoek aangaande een 35 patiënten die een complex buikwandhernia herstel hebben ondergaan met als doel de correlatie tussen hernia volume, gemeten op abdominale CT-scan, en het optreden van pulmonale complicaties te achterhalen. Er blijkt een duidelijke correlatie te zijn tussen het optreden van pulmonale complicaties en de grootte van de ventrale buikwandhernia. Kortom, hoe groter de ventrale buikwandhernia, hoe groter het risico op pulmonale complicaties.

\section{INTRA-OPERATIEF}

Hoofdstuk 4 Beschrijft een endoscopische modificatie van een operatietechniek voor ventraal hernia herstel, de endoscopisch geassisteerde component separatie techniek (ECST). De techniek is een moderne variatie op de component separatie techniek beschreven door Ramirez in 1990. Met deze techniek wordt getracht het aantal wondcomplicaties te verkleiner door gebruik te maken van een kleiner wondbed in endoscopische pockets. De resultaten van een groep van 27 patiënten zijn veelbelovend, en beter dan de tot nu toe beschreven resultaten van de klassieke operatie. 
Hoofdstuk 5 Beschrijft een modificatie van een in de jaren 70 geïntroduceerde onlay procedure genaamd de Chevrel techniek. Deze relatief eenvoudige techniek kan worden gebruikt om complexe hernia's op een gemakkelijke manier te herstellen. In dit hoofdstuk wordt de techniek in detail uitgelegd en worden de resultaten van deze techniek bij 155 patiënten geëvalueerd. De gemodificeerde Chevrel techniek leidt tot een hoge kwaliteit van leven voor de patiënt en wordt gekenmerkt door weinig recidieven. Wel is er spraken van een hoger postoperatief complicatie percentage (23.2\%), met name doordat seromen na deze techniek makkelijker worden gediagnosticeerd in vergelijking met sublay of intra-abdominale mesh plaatsingen.

Hoofdstuk 6 Beschrijft een vergelijkende dierstudie waarbij een viertal meshes in onlay positie (subcutaan) zijn ingebracht bij tachtig mannelijke Whistar Han ratten. De studie heeft als doel de klinische eigenschappen tussen deze vier veelgebruikte meshes te analyseren. Er wordt geen verschil gemeten ten aanzien van de ingroei van de mesh. Er wordt wel geobserveerd dat er een duidelijk verschil zit in het krimpen van de mesh na implantatie. De polyester mesh krimpt nagenoeg niet, terwijl de polypropyleen meshes, afhankelijk van het type tot wel 10\% krimpen ten opzichte van baseline.

Hoofdstuk 7 Beschrijft een patiënt welke een nieuw soort oplosbare mesh heeft ontvangen en postoperatief wordt verdacht van een geïnfecteerd seroom op de mesh. Deze situatie zou bij gebruik van een standaard synthetische mesh zeer waarschijnlijk eindigen in een mesh infectie, hetgeen het verwijderen van de mat noodzakelijk zou maken. Deze patiënt wordt gepresenteerd in een case report omdat er gedurende het conservatieve beleid ten aanzien van het seroom een volledig herstel wordt geobserveerd zonder noodzaak tot chirurgische interventie.

Hoofdstuk 8 Onderzoekt de chirurgische en fysiotherapeutische behandelmogelijkheden van een veel voorkomend probleem zijnde rectus diastase. Deze aandoening ontstaat meestal tijdens de zwangerschap doordat de middellijn (ruimte tussen de rectus abdominis spieren) verbreed in combinatie met zwakte van de ventrale buikwandmusculatuur. Er wordt een uitgebreide analyse gedaan naar de mogelijke behandelingen van rectus diastase waaruit blijkt dat indien chirurgie gewenst is vaak wordt gekozen voor een open of laparoscopische plicatie van de middellijn met mesh versteviging. Indien er ook sprake is van een kleine hernia wordt gekozen voor een aangepaste hernia herstel techniek met mesh versteviging. Een succesvolle behandeling van rectus diastase middels fysiotherapie is nog niet beschreven in de huidige literatuur, enkel een geringe reductie van de middellijn breedte tijdens spiercontractie, waarvan het onduidelijk is of dit cosmetische of functionele voordelen voor de patiënt oplevert. 


\section{POSTOPERATIEF}

Hoofdstuk 9 Gaat in op het meten van door de patiënt zelf gerapporteerde uitkomstmaten (PROMs) middels een nieuwe kwaliteit van leven vragenlijst, de COMI-hernia vragenlijst, in een populatie van 120 liesbreuk patiënten voor en na een laparoscopisch totaal extraperitoneale liesbreuk plastiek. In dit hoofdstuk wordt de eerste klinische ervaring van deze vragenlijst beschreven, buiten de originele publicatie van de ontwikkelaars. De kwaliteit van leven in deze populatie is grotendeels conform eerder beschreven publicaties, doch lijkt er spraken van hoger percentage postoperatieve pijn dan verwacht. De COMI-hernia vragenlijst werkt goed maar de interpretatie van de vragenlijst is lastig, mogelijk door een gebrek aan ervaring en/of overlap in de geëvalueerde uitkomstmaten.

Hoofdstuk 10 Beschrijft de toepassing van de Delphi techniek om internationale consensus te bereiken over het scoren van postoperatieve mesh gerelateerde adhesies. Op basis van de resultaten beschreven in dit hoofdstuk is een nieuwe adhesie score ontwikkeld en worden er aanbevelingen gedaan betreffende de methodologie en het meten van uitkomstvariabelen in mesh gerelateerd adhesie onderzoek.

Hoofdstuk 11 Beschrijft een retrospectief single center cohort van patiënten met Ehlers Danlos. Deze groep patiënten heeft een verstoorde collageen synthese waardoor algemeen wordt aangenomen dat zij een groot risico hebben op het ontstaan van zowel primaire als recidief buikwandbreuken. Het cohort betreft veertien patiënten met Ehlers Danlos van verschillende typen, welke allen een hernia herstel hebben ondergaan. Tijdens deze procedure is er een extra grote mesh geplaatst om de lagere collageen kwaliteit te compenseren. Opvallend is dat het recidiefpercentage na 5 jaar mediane follow-up van 7\%, lager is dan het vanuit de literatuur bekende recidiefpercentage $(12 \%)$ in de algemene bevolking na een mediane follow-up van 59 maanden.

Hoofdstuk 12 Onderzoekt de basis prestatie van het ischemic button model in zowel mannelijke en vrouwelijke Whistar Han ratten. Er blijkt geen verschil te zijn in de hoeveelheid geïnduceerde adhesies, nog de kwaliteit van de geïnduceerde adhesies. De mannelijke ratten nemen significant sneller in gewicht toe, ook wanneer de gewichtstoename wordt gecompenseerd voor fysiologische groei. Er is geen verschil in additionele pijnstilling of welfare score tussen de mannelijke en vrouwelijke proefdieren. Het verschil in gewichtstoename kan worden verklaard door dimorfisme in pijnbeleving en pijnbestrijding tussen beide seksen. 



\section{Acknowledgements / Dankwoord}

Writing this thesis has not been, as one might expect, solely a scientific exercise, it has changed who I am as a person, a friend, a scientist, and a doctor. Looking back, I now realise that the people 'surrounding me' have had a profoundly positive influence on my career and life choices. Without the necessary role models and stimulating words of others, my attempts in science would have surely been futile. For this reason, I consider this thesis not a personal achievement, but rather a privilege given to me by others through the investment of their time and energy. It is through their efforts they have created an environment in which I could safely develop and improve my scientific skills. For this reason, I can't finish this thesis without thanking them.

Het promotietraject heeft mij doen inzien dat de mensen die zich 'om mij heen' bevinden bewust en onbewust een grote (positieve) invloed hebben uitgeoefend op mijn persoonlijke en carrière keuzes. Zonder de voorbeeldrol en de stimulerende woorden van anderen zou mijn poging voor een doctoraat zeker zijn gestrand. Opmerkelijk vind ik het proces waarmee dit heeft plaatsgevonden, aangezien de contacten met enkele van de meest invloedrijke personen in mijn omgeving vaak spontaan leken te ontstaan. Het is om die reden dat ik het behalen van dit doctoraat niet enkel zie als een persoonlijke prestatie, maar eerder als een eer en een voorrecht dat mij door de investering van tijd en energie door anderen is gegund. Zij hebben de omgeving gecreëerd waarin ik mijn wetenschappelijke kennis en kunde heb kunnen ontwikkelen. Met inachtneming van het risico om mensen te vergeten, buiten te sluiten of voor het hoofd te stoten zijn er enkele belangrijke mensen die ik in het bijzonder wil bedanken voor hun bijdrage.

Dr. Tammo S. de Vries Reilingh, Beste Tammo, jij was het die mij introduceerde in de wereld van de hernia chirurgie. Op jouw werkkamer legde je me de methodiek van de endoscopisch geassisteerde Ramirez plastiek uit. Een begin dat uiteindelijk een stip op de horizon zette voor een thesis. Het is met jouw begeleiding geweest dat we deze stip hebben kunnen bereiken en daarvoor ben ik je zeer dankbaar. Op jouw geheel eigen manier heb je mij bijgestaan voor, tijdens en na mijn periode als ANIOS in het Elkerliek ziekenhuis. Ik had me geen betere begeleider kunnen wensen. Je hebt ondanks je drukke schema in de perifere kliniek altijd tijd gemaakt voor me. Om tien uur's avonds een telefoontje om te vragen hoe de projecten ervoor stonden en op vakantie de telefoon beantwoorden in de skilift was jou niet vreemd. Alleen dat koffie halen heb je me ondanks verwoede pogingen niet kunnen aanleren. Ik zal blijven oefenen. Deze thesis was er niet gekomen zonder jouw inspiratie, je bijdrage en je motivatie. Dank!

Dr. Simon W. Nienhuijs, Beste Simon, jouw ervaring als onderzoeker en begeleider heeft mijn promotietraject naar een hoger niveau getild. Vanaf het begin heb jij op de achter- 
grond de lijn uitgestippeld en de kwaliteit van het onderzoek gewaarborgd. Milou en ik denken met veel plezier terug aan de kleine 'acts' in je repertoire die de stille momenten in de kroeg of tijdens een diner kunnen opvullen. Ik heb je in de afgelopen jaren regelmatig horen zeggen dat je 'als je 100 pubmed hits hebt bereikt' je zal stoppen met het doen van onderzoek. Op het moment van schrijven staat de teller precies op honderd. Maar zoals bekend willen gestelde grenzen nogal eens schuiven. Ik ben dan ook blij om te horen dat je toch doorgaat in de wetenschap, het zou een groot gemis zijn als je was gestopt. Dankjewel voor de enthousiaste begeleiding, dank voor je recht door zee benadering van problemen en dank voor de vrijheid die je me hebt gegeven in het onderzoek.

Professor Dr. Nicole D. Bouvy, Beste Nicole, in de fase dat ik verdieping en versnelling van mijn onderzoek zocht was $u$ degene die mij verwelkomde binnen uw onderzoeksgroep. Niet alleen gaf $u$ mij de mogelijkheden om mijn eigen onderzoek te continueren, $\mathrm{u}$ stelde ook de middelen en voorzieningen van de onderzoeksgroep ter beschikking met de woorden 'dit is nu ook jouw speeltuin, maak er iets moois van'. Met veel enthousiasme en vol spontaniteit heeft u mijn promotietraject samen met de copromotoren begeleid. Ik benijd uw energie en vermogen tot het combineren van een gezin, medisch specialist en hoogleraarschap innovatieve chirurgie. Bovenal waardeer ik de manier waarop u mij hebt begeleid, door op een gelijkwaardig niveau de zaken te bespreken en als macromanager het proces te overzien. Ik roem je positieve mindset, je onvermoeide inzet voor je patiënten en je promovendi, je openhartigheid, je vermogen om tijd te creëren in een overvolle agenda en je multitask vaardigheden. Als er meer mensen zoals jij in het ziekenhuis rondliepen gingen de zaken een stuk soepeler. Dank voor je vertrouwen, en dank voor begeleiding!

Drs. Jan Charbon, Beste Jan, samen hebben we een integraal hoofdstuk in deze thesis geschreven over een gemodificeerde Chevrel techniek. Jouw aandacht voor detail heeft ons project goed gedaan en de tijd en energie die je daarin hebt gestopt zijn lovenswaardig. Je hebt niet alleen gecoördineerd, maar we hebben samen dagenlang de database gevuld, de analyses gedaan en het stuk vorm gegeven. Zonder jouw inspanning en begeleiding was het artikel er niet gekomen. Dank voor de fantastische samenwerking!

Drs. Johannes A. Wegdam, Beste Johannes, in de tijd na mijn afstuderen heb je een grote rol gespeeld in het faciliteren van mijn onderzoekstraject, daarvoor ben ik je zeer dankbaar. Zonder de mogelijkheden die jij mede hebt gecreëerd had dit traject er heel anders uit gezien. Jouw vermogen om op een positieve manier langs torenhoge obstakels te kijken is een inspiratie in donkere tijden. De ' $s$ ' in jouw titel is al jou al jaren een doorn in het oog en een uitstekende motivatie voor jouw eigen promotietraject. Op basis van alle hoofdstukken met jou als coauteur kan ik nu met zekerheid zeggen dat het begin is gemaakt. Ik wens je veel succes met het schrijven van je thesis, en ik dank je voor je ondersteuning. 
Dr. Guy J.C.M. Niesen, Beste Dr. Niessen, in mijn tijd als arts-assistent was u degene die de standaard hooghield. In de kliniek was $u$, als meest ervaren staflid, bereid om iedereen iets te leren. $U$ was degene die altijd tijd maakte voor klinische begeleiding. Ook tijdens mijn onderzoeksdagen was $\mathrm{u}$ altijd in voor een relativerend gesprek. Veel dank.

Drs. Marc Guijt, Beste Marc, dankjewel voor de begeleiding tijdens mijn tijd in het Elkerliek en veel dank voor de vergevingsgezindheid met betrekking tot je aanstelling en de spelling van je achternaam. Dank voor je inspirerende ideeën en de begeleiding in het Elkerliek ziekenhuis.

Drs. Wendy Bos, Drs. Bea Lemaire, Drs. Jan Bernard Sintenie, Drs. Christiaan de Groot, Drs. Guido Stultiëns, Beste Wendy, Bea, Jan Bernard, Christiaan en Guido, dank jullie wel voor het vertrouwen in mij in mijn tijd als ANIOS in het Elkerliek ziekenhuis, en het gunnen van mijn zo gewaardeerde 'onderzoekstijd'. Het was een ideale gelegenheid om mijn onderzoek voort te zetten en een goede leerschool. Bedankt!

Drs. Heidi van den Hoven, Drs. Monique Foppen, Beste Heidi en Monique, Ik wil jullie bedanken voor jullie begeleiding tijdens mijn tijd in het Elkerliek ziekenhuis. Zonder jullie ondersteuning had ik de tijd niet gehad om mijn thesis te kunnen verdiepen in het Elkerliek ziekenhuis. Ik wens jullie alle goeds.

Drs. Dorien R.M. Hünen, Beste Dorien, jij hebt me toegelaten tot jouw onderzoek, hetgeen uiteindelijk een hoofdstuk in dit boekje is geworden. Ik wil je bedanken voor je bijdrage aan mijn thesis en wens je veel succes in je verdere carrière als huisarts.

Collega's van de onderzoeksafdeling heelkunde. Dank voor alle mentale ondersteuning, het scheppen van een gezond en afwisselend werkklimaat, de constructieve input en de ondersteuning van mijn onderzoeksplannen. Ik ben blij dat ik de verhuizing naar de 'nieuwe' afdeling heb mogen meemaken, het heeft geresulteerd in een afdeling die past bij jullie status en ambitie. Beste Mo en Bas, zonder jullie zou ik nu nog verdwaald zijn op het lab, zoekend naar spullen of methoden om mijn resultaten te bemachtigen. Beste Claire, Frans, Cathelijne, dankjewel voor jullie hulp.

Drs. Leontine van den Hil, Beste Leontine, dankjewel voor je advies en begeleiding bij mijn start in het MUMC. Dank voor al die uren samen 'even' overleggen en dank voor al je collegialiteit en respect. Samen hebben we de fundering gelegd voor vele jaren aan onderzoek. We kunnen trots zijn op het resultaat! Ik wens je veel succes in je verdere chirurgische carrière!

Drs. Hong Liu, dear Hong, I consider you a friend and I wish you all the best in your future career. I have the upmost respect for the way you handled your transition to a foreign country, being separated from you friends and family for quite a while. I wish you all the best in your research career and hope to hear from you soon. 
Drs. Sebastiaan van Steensel, Beste Sebastiaan, aan jou de taak om het onderzoek voort te zetten. Je hebt direct veel hooi op de vork genomen toen je op de afdeling kwam en daarmee is de weg naar een razendsnelle promotie ingezet. Ik twijfel niet aan je ambitie en wens je veel succes in zowel je research carrière en in je verdere loopbaan als chirurg.

Dr. Kevin A.T.M. Theunissen, beste Kevin, jij weet door jouw promotietraject als geen ander wat afzien is, ondanks dat heb je vol gehouden en een boekje afgemaakt waar je trots op mag zijn. Die inspanning heeft voor mij altijd als motivatie gediend. Je hebt altijd voor me klaar gestaan, al sinds de tijd dat we samen bij foto Rembrandt werkten. Je bent altijd bereid om afleiding te bieden in goede en slechte tijden. Ik voel me bevoorrecht met een vriend zoals jij. Dank dat je me als paranimf ook bij deze beproeving wilt bijstaan.

Drs. Lars P.W. Mommers, Lieve Lars, als oudere broer en begenadigd anesthesistintensivist ben jij een grote inspiratie voor me. Er zijn maar weinig mensen waar je blindelings op kunt vertrouwen, maar voor mij ben jij zo iemand. De wetenschap dat ik altijd op je kon rekenen, zaken met je kon overleggen en bij je kon aankloppen voor advies en reflectie heeft deze reis een stuk minder zwaar gemaakt. Uiteraard verricht ik graag dezelfde dienst in de ondersteuning bij jouw promotietraject, maar ik twijfel geen moment dat je die net als alle andere academische beproevingen die je hebt ondervonden, glansrijk zult afronden. Uit de grond van mijn hart, dankjewel.

Dr. Milou H. Martens, Lieve Milou, de voornaamste reden om te beginnen aan dit traject is zodat wij weer een gelijkwaardig gesprek kunnen voeren aan de eetkamertafel. Zonder jouw inspiratie, doorzettingsvermogen en ogenschijnlijk gemak waarmee je over intellectuele uitdagingen heen springt was ik nooit begonnen aan dit traject. Dankjewel voor je onvoorwaardelijke steun, je opbouwende commentaar en je (tot nu toe) onuitputbare vermogen om mij te tolereren op mijn slechtste momenten. Ik voel me bevoordeeld met jou naast me, door jou ben ik een beter mens. Ik hoop dat we nog veel uitdagingen samen mogen aanpakken.

Lieve ouders, stiefouders, schoonfamilie, familie, dank voor het creëren van een veilige omgeving, dank voor de financiële en emotionele steun tijdens de opleiding en tijdens dit promotie traject. Dank voor de niet aflatende interesse in mijn onderzoek ondanks dat de antwoorden op vragen als 'waar ben je nu mee bezig?' vaak erg langdradig waren.

Aan iedereen die in de afgelopen jaren nietsvermoedend heeft gevraagd 'hoe gaat het' en vervolgens geduldig heeft geluisterd naar eindeloze verhalen over hernia's, adhesies en onderzoeksperiekelen, weet dat hij voor mij veel heeft betekend. Dank. 


\section{List of publications}

\section{THIS THESIS}

Mommers EHH, Hunen DR, van Hout JC, et al. Patient-reported outcomes (PROs) after total extraperitoneal hernia repair (TEP). Hernia: the journal of hernias and abdominal wall surgery 2017;21(1):45-50.

Mommers EHH, Wegdam JA, Nienhuijs SW, de Vries Reilingh TS. How to perform the endoscopically assisted components separation technique (ECST) for large ventral hernia repair. Hernia: the journal of hernias and abdominal wall surgery 2016;(20):441-7.

Mommers EHH, Wegdam JA, van der Wolk S, Nienhuijs SW, de Vries Reilingh TS. Impact of hernia volume on pulmonary complications following complex hernia repair. Journal of surgical research 2017;(211)8-13.

Mommers EHH, Leenders BJM, Leclercq WKG, de Vries Reilingh TS, Charbon JA. A modified Chevrel technqiue for ventral hernia repair: long-term results of a single center cohort. Hernia: the journal of hernias and abdominal wall surgery 2017 April 13. [Epub ahead of print]

Mommers EHH, Ponten JEH, Al Omar AK, de Vries Reilingh TS, Bouvy ND, Nienhuijs SW. The general surgeon's perspective of rectus diastasis. A systematic review of treatment options. Surgical endoscopy 2017 June $8^{\text {th }}$ [Epub ahead of print]

Kroese LF, Mommers EHH, Robbens C, Bouvy ND, Lange JF, Berrevoet F. Complications and recurrence rates of patients with Ehlers Danlos syndrome undergoing ventral hernioplasty. Accepted in Hernia January 2018

\section{OTHER}

Hannemann PF, Mommers EHH, Schots JP, Brink PR, Poeze M. The effects of lowintensity pulsed ultrasound and pulsed electromagnetic fields bone growth stimulation in acute fractures: a systematic review and meta-analysis of randomized controlled trials. Archives of orthopaedic and trauma surgery 2014;134:1093-106.

Janssen AH, Mommers EHH, Notter J, de Vries Reilingh TS, Wegdam JA. Negative pressure wound therapy versus standard wound care on quality of life: a systematic review. Journal of wound care 2016;25:154, 6-9. 


\section{SUBMITTED}

Mommers EHH, Hong L, Jongen A, Bouvy ND. Sex differences in baseline performance of the ischemic button model for induction of adhesions in laboratory rats. Submitted

Mommers EHH, Barneveld KWY, Gijbels MJ, de Vries Reilingh TS, Schreinemacher MHF, Bouvy ND. Evaluating the 'standard' polypropylene and polyester mesh in terms of shrinkage, tissue ingrowth, and collagen deposition. Submitted

Mommers EHH, Van Vugt LK, Bouvy ND, Wegdam JA, de Vries Reilingh TS. Use of a bio absorbable mesh for complex ventral hernia repair in a contaminated field: a case report. submitted 


\section{Curriculum Vitae}

Elwin Henri Hubert Mommers was born on the $29^{\text {th }}$ of January 1990 in Maastricht, the Netherlands. After completing grammar school at the Porta Mosana College in Maastricht he studied health sciences at Maastricht University. In 2009 his interest in the medical profession motivated him to start the Bachelor of Medicine.

During his internships in 2013 at the Elkerliek Hospital in Helmond, Dr. Tammo S. de Vries Reilingh introduced him to abdominal wall hernia repair for the first time, this turned out to be the beginning of a thesis in abdominal wall repair.

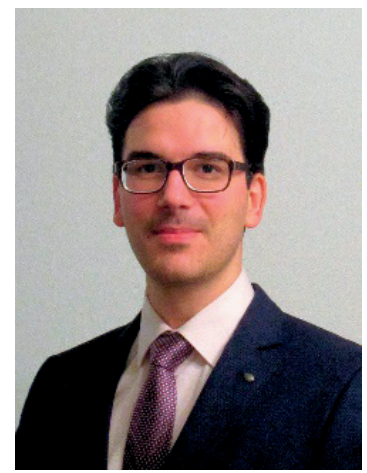
His primary work concerned surgical techniques for ventral hernia repair and included detailed descriptions of the Endoscopically assisted Component Separation Technique and the modified Chevrel techniques. These studies were the foundation for his later work on the surgical management of rectus diastasis and the identification of novel risk factors for postoperative complications. After completing the prestigious honours programme research, he graduated from medical school in 2015. Eager to continue his research he obtained a combined surgical resident/research position at the Elkerliek hospital Helmond. With the guidance of Dr Tammo S. de Vries Reilingh and Dr. Simon W. Nienhuijs he continued, and extended, his research in ventral hernia repair until transferring to the research group of Prof. Dr. Nicole D. Bouvy at the Maastricht University Medical Center (MUMC+) in 2016. After the transfer, the focus of his research widened from hernia repair to intra-abdominal adhesion formation, two areas that are often entangled.

During 2017, the diversity of clinical problems and the techniques in the medical imaging department caught his attention. Eager to start work in a new scientific field and broaden his personal and scientific horizon, he could not resist the charms of the radiology department where in July 2017 he started his residency. 



\section{Valorisation}

Why haven't we solved one of the most frequent complications of abdominal surgery?

Any 'number' surrounding abdominal wall hernias is impressive, whether it is the three million repairs performed annually throughout the world, the eleven to thirty-nine percent recurrence rate after repair, the six-billion-dollar expendable market surrounding surgical intervention or the ten percent emergency procedures necessary to prevent serious illness or even death. It are these disturbing numbers that justify scientific research in this important field. A hernia consists of three defined anatomical structures, the hernia defect, hernia sac, and hernia contents. Managing any type of hernia consists of three equally important phases, the preoperative phase, intraoperative phase, and postoperative phase. The complexity of the hernia, combined with the patient characteristics will increase of decrease the complexity of each management phase. 'Standard' hernias, such as a small umbilical hernia, will require less preoperative planning compared to a large incisional hernia with loss-of-domain. The origin of this thesis was born out of necessity, as managing complex hernias requires not only a clinical performance, though also an academic investment to evaluate and reflect the obtained results. This thesis was not aimed at developing any type of commercial product. On the contrary, it is a call for less commercial products, a testament to previous work, a chance for reflection, and an exploration of recent advances in ventral hernia repair.

\section{TARGET POPULATION}

The academic field of hernia repair consists of several sub domains, classified according to the anatomical location of the hernia. Inguinal hernias are most common and make up $75 \%$ of the field, followed by femoral hernias, umbilical hernias, epigastric hernias, and incisional hernias in decreasing order of incidence. Inguinal hernias frequently occur in elder males, epigastric hernias have no gender preponderance, and incisional hernias are essentially a complication from surgery, though age and male gender are known risk factors.

The focus of this thesis is on ventral hernias, a combination of primary and incisional hernias, including epigastric, umbilical, and suprapubic, hernias form approximately 10$15 \%$ of all hernias. Hence, the socioeconomic relevance of this thesis lies in providing the best possible care for these patients. This includes any preoperative, intraoperative, and postoperative decisions made regarding the treatment of ventral hernias. 


\section{RELEVANT OUTCOMES OF THIS THESIS}

Despite any scientific gains resulting from this thesis, it is important to keep the patients' perspective in mind. Far too often doctors are concerned with the type of mesh, or the type surgical technique suitable for treating a hernia. Although these are valid considerations, patients are usually not very interested in different types of surgical mesh, nor are they interested in the technical approach. They want to know how long it takes before the pain goes away, if there is a change of chronic pain, what the cosmetic outcome will be, and when they can go back to work. These are examples of patient reported outcome measures. One would expect that this type of outcome is used frequently in scientific research, yet the opposite is true. A 2014 high-impact review states that between 2008 and 2012 only 16\% of high-impact articles in general surgery reported at least one patient reported outcome (PRO). Only ten of these articles concerned hernia repair. This thesis includes a publication with a new type of PRO-questionnaire specifically designed for measuring PROs in a hernia population.

Despite the surgeon's best efforts, complications are inevitable. Though the risk of postoperative complications in not equal for all patients. This thesis describes a new diagnostic device (the electronic nose) that can be used to identify patients that are at high risk for a recurrence of their ventral hernia. Although the evidence is still low grade and more research is needed to determine the translatability to the clinic and confirm the accuracy of the proof-of-concept study, the idea of implementing the electronic nose in the field of hernia repair is promising. The device measures volatile organic compounds in exhaled air to determine if patients are at risk for a certain disease. The proof-of-concept study included in this thesis shows that the eNose can accurately differentiate recurrent hernia patients from healthy controls, as well as aortic aneurysm patients from healthy controls.

Alongside the groundwork for eNose diagnostics and hernia volume as a risk factor for pulmonary complications, this thesis includes three detailed technique descriptions, two regarding ventral hernia repair and one regarding rectus diastasis. The latter may not be a true ventral hernia, though patients are frequently referred to the surgery outpatient clinic for treatment. This thesis provides some recommendations for treating rectus diastasis and has found that physiotherapy for rectus diastasis is not an evidencebased therapy at this moment.

\section{INNOVATION \& FUTURE}

Hernia repair is slowly moving towards centralisation, in which the straightforward cases are treated in regular clinics and complex cases are centralised in specialised clinics. Though currently there is a lack of standardisation to facilitate the selection of straightforward repairs and define complex cases. The results of this thesis, with the 
description of several surgical techniques such as the Endoscopically assisted Component Separation Technique (ECST), the modified Chevrel technique for complex hernia repair, and the identification of new risk factors (Hernia volume) and innovative diagnostic tools (eNose) can help in the specialisation process. Dedicated hernia repair centers must have several surgical approaches readily available for treating complex hernias, including standard and 'go-to' techniques. One of the most frequently used techniques for open complex hernia repairs is the Rives-Stoppa approach with a sublay mesh. Though experienced centers must have techniques, such ECST and modified Chevrel techniques, in their repertoire in case the standard technique does not allow midline closure. The primary goal of ventral hernia repair should be midline closure with mesh reinforcement. Bridging repairs should only be used in case midline closure is not possible. Recent studies indicate that laparoscopic repairs should also attempt to close the hernia defect to prevent seroma formation and other adverse surgical site occurrences.

During the following years the most important development in the field of ventral hernia repair will not be a surgical technique such as Botox, or a commercial development such as a new type of mesh. Instead, the most important innovation will be the development and implementation of evidence and expert opinion-based guidelines for ventral hernia repair. Implementing guidelines will facilitate the division of 'standard' and 'complex' cases and aid the development of specialised clinics. Moreover, standardisation in the field of ventral hernia repair will increase interstudy comparability, as the multitude of different meshes, techniques, and fixation methods is currently reducing the translatability and comparability of scientific research. Unifying the field of hernia research can drastically increase the quality of the scientific output of the hernia community, as well as provide better guidance to the commercial industry surrounding ventral hernia repair. 
